\title{
Untersuchung der lokalen strukturellen und elektronischen Eigenschaften von Fe-GaAs Schottky-Kontakten mit atomar aufgelöster Raster-Tunnel-Mikroskopie in Querschnittsgeometrie
}

\author{
Dissertation \\ zur Erlangung des Doktorgrades \\ der Mathematisch-Naturwissenschaftlichen Fakultäten \\ der Georg-August-Universität zu Göttingen \\ vorgelegt von \\ Lars-Helge Winking \\ aus Lübeck
}

Göttingen, 2009 
D7

Referent: Prof. Dr. R.G. Ulbrich

Korreferent: Prof. Dr. R. Kirchheim

Tag der mündlichen Prüfung: 29. Januar 2009 


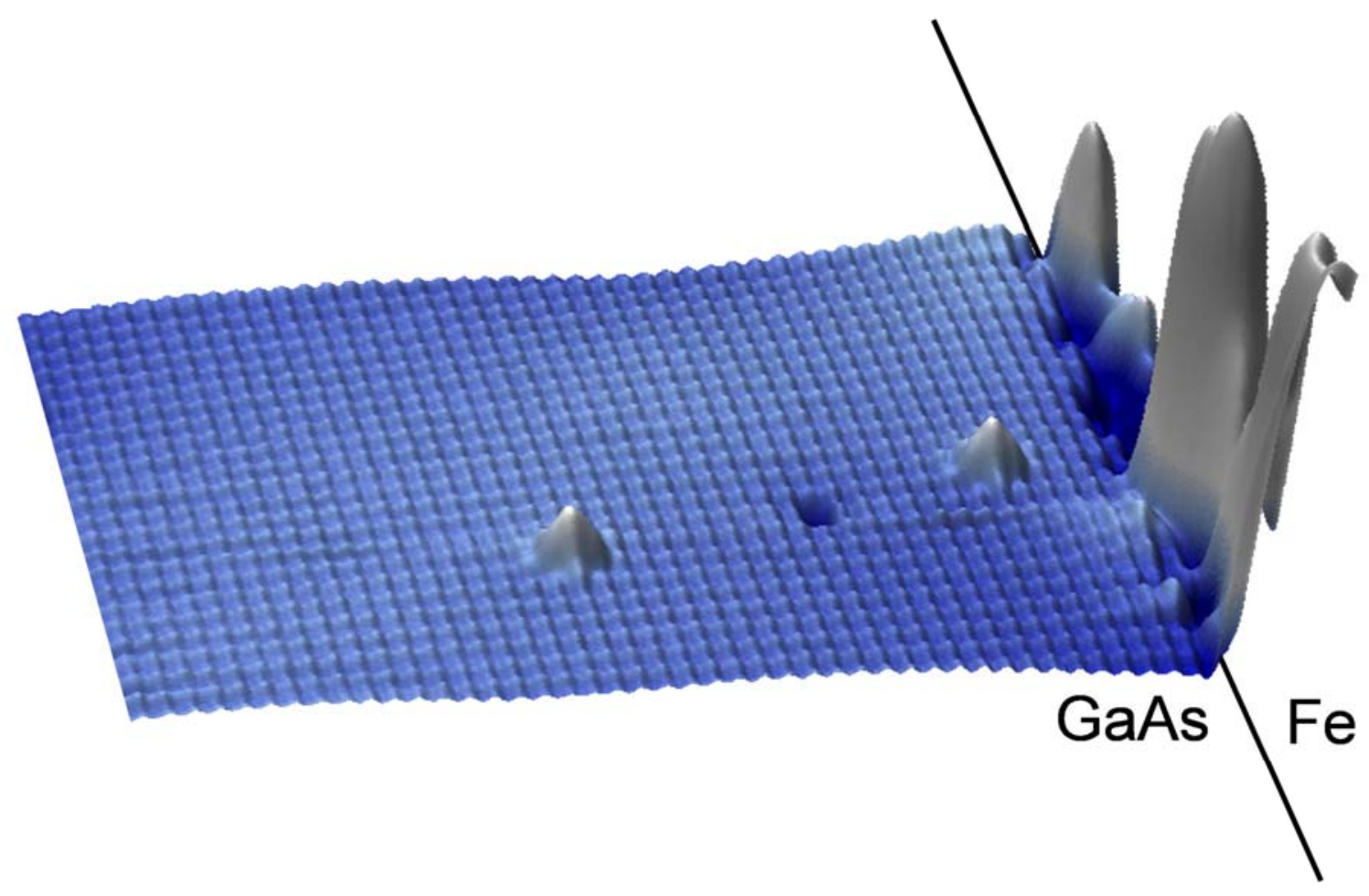

Abbildung 1: Atomar aufgelöste XSTM Topografie aus dem Bereich der Fe-GaAs(110) Grenzfläche in einer 3D-Darstellung. Der Bereich des GaAs Substrats hebt sich durch die atomare Korrugation deutlich von dem Bereich des unregelmäßig gespaltenen Fe-Films ab. Vereinzelte atomare Defekte wie Fehlstellen und Adatome sind deutlich zu erkennen.

\section{Vorwort}

Die Ausbildung einer gleichrichtenden Barriere an Metall-Halbleiter Grenzflächen hat seit weit mehr als 100 Jahren das Interesse einer Vielzahl von Wissenschaftlern auf sich gezogen [1, 2]. Um zu erklären, welche Mechanismen die Ausprägung dieser Schottky-Barriere bestimmen, wurden zahlreiche Modelle vorgeschlagen und intensiv diskutiert [3-13]. Dennoch gibt es bis heute keine allgemein anerkannte Theorie, die die enorme Menge an experimentellen Daten zufrieden stellend erklären kann. Dies hängt entscheidend damit zusammen, dass in fast allen Studien, die sich mit der Erforschung von Metall-Halbleiter Kontakten auseinandergesetzt haben, wichtige Details über die Struktur, die Stöchi- 
ometrie und insbesondere Defekte an der Grenzfläche nicht ausreichend bekannt sind. Die Gründe dafür sind vielfältig. Zum einen fehlte bis zur Entwicklung der Ultra-Hoch-Vakuum (UHV) Technologie die Möglichkeit, wohl definierte epitaktische Metall-Halbleiter Systeme herzustellen [14]. Aber auch nach Einführung der UHV Technik ist es aufgrund der Komplexität der Heteroepitaxie nur bei wenigen Materialsystemen wie $\mathrm{NiSi}_{2}$-Si $[15,16]$ oder Al-GaAs [17] gelungen, ideale SchottkyKontakte mit abrupten, homogenen Grenzflächen zu präparieren. Dementsprechend wurden bislang in nahezu allen Studien Systeme mit polykristallinen Metallfilmen verwendet, die zwar technologisch relevant sind, sich aber wegen ihrer inhomogenen Grenzfläche nur sehr bedingt eignen, um die fundamentalen Mechanismen der Schottky-Barrierenformierung zu untersuchen [18-20]. Zum anderen hat sehr lange eine Methode gefehlt, mit der man Schottky-Kontakte im Realraum mit Nanometer Auflösung untersuchen konnte. Ein wichtiger Schritt zur Beseitigung dieser Einschränkung bestand in der Einführung der Ballistischen-Elektronen-Emissions-Mikroskopie (BEEM) in den frühen 90er Jahren, mit der erstmals die laterale Variation der Schottky-Barrierenhöhe (SBH) auf der Nanometerskala nachgewiesen werden konnte [21-23]. Da die BEEM Methode allerdings keine Aussagen über die strukturellen Eigenschaften des untersuchten Schottky-Kontakts erlaubt, existiert bislang noch immer keine Studie, bei der insbesondere der Grenzflächenbereich im Realraum strukturell und elektronisch auf der atomaren Skala untersucht werden konnte.

Dabei ist ein grundlegendes Verständnis der Ausbildung einer Schottky-Barriere mehr als nur von akademischem Interesse. Schottky-Kontakte sind mittlerweile ein wichtiger Bestandteil von nahezu allen halbleiterbasierten elektronischen und optoelektronischen Bauteilen. Sie müssen dabei eine Reihe von Voraussetzungen in Bezug auf ihre elektronischen, chemischen und auch mechanischen Eigenschaften erfüllen. Dazu zählen sowohl die Kontrollierbarkeit, die Uniformität und die Reproduzierbarkeit der SBH als auch die chemische Stabilität unter Umgebungs-bedingungen wie zum Beispiel thermischen Belastungen des Bauelements [24]. Ein besseres grundlegendes Verständnis der physikalischen Zusammenhänge, die die Eigenschaften von Metall-Halbleiter Kontakten bestimmen, kann in diesem Kontext einen entscheidenden Beitrag dazu leisten, um den Anforderungen gerecht zu werden. Die voranschreitende Miniaturisierung moderner Halbleiterbauelemente, die bereits 1965 von Moore vorhergesagt wurde, wird darüber hinaus in Zukunft neue und immer höhere Ansprüche an die einzelnen Komponenten stellen [25]. Die „International Technology Roadmap for Semiconductors 2007“ sagt voraus, dass die Strukturgrößen in Feld-Effekt Transistoren (FET) wie Schottky-FETs, bei denen ein Schottky-Kontakt als Steuerelektrode (engl. gate) verwendet wird, im Verlauf der nächsten 10 Jahre den Bereich von $10 \mathrm{~nm}$ erreichen werden [26]. Dies bedeutet, dass neben der Kontrolle der systemspezifischen SBH vor allem die stochastische Verteilung von Dotieratomen in der Raumladungszone (RLZ), die zu natürlichen Inhomogenitäten der effektiven Barrierenhöhe führt, immer stärker über die makroskopischen Eigenschaften eines solchen Bauteils entscheiden wird [27, 28]. 


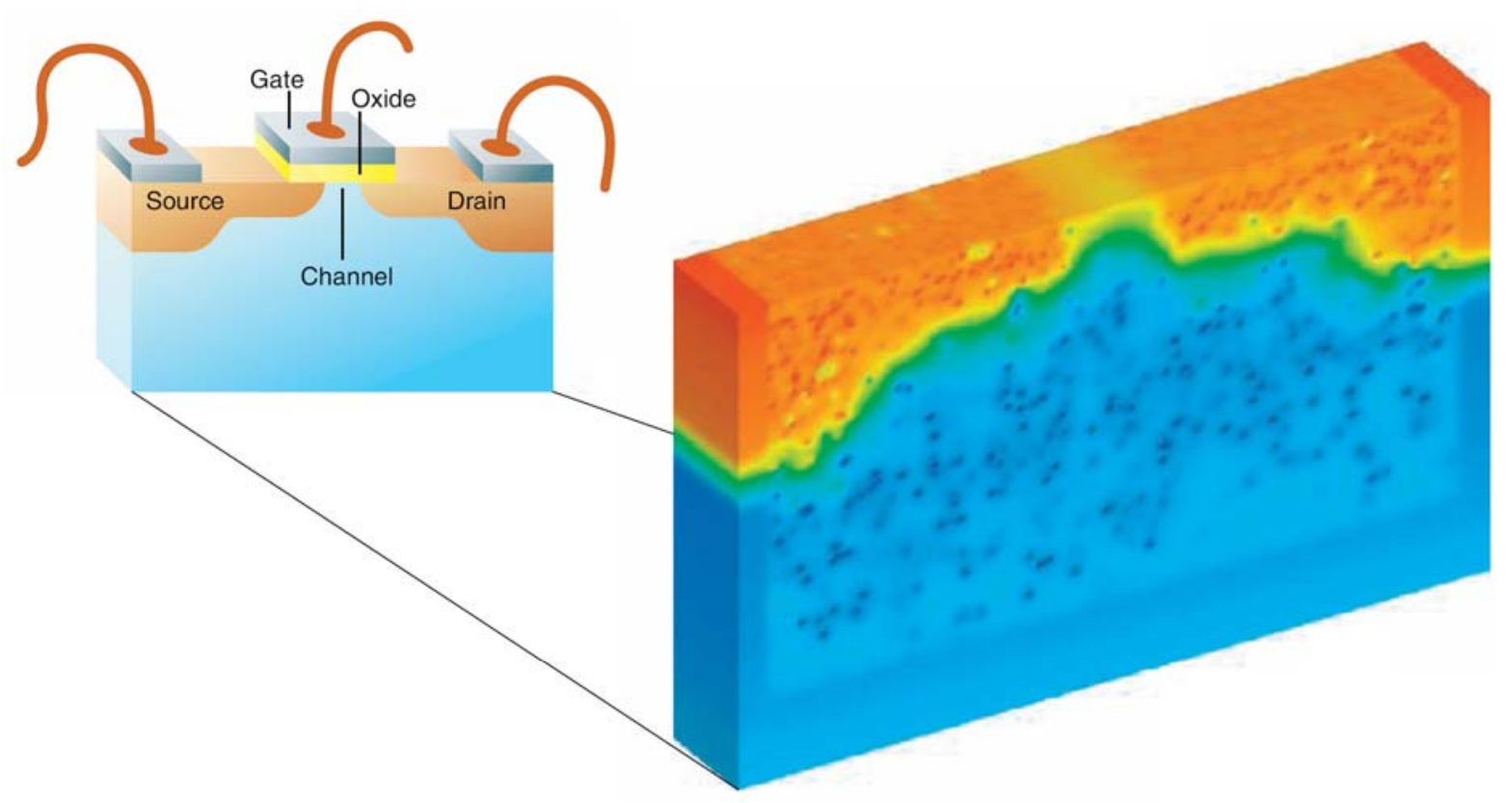

Abbildung 2: 3D-Simulation eines $(30 \times 30) \mathrm{nm}^{2}$ großen Feld-Effekt Transistors mit einer stochastischen Verteilung von Dotieratomen in den Bereichen von Quelle (engl. source), Kanal (engl. channel), Senke (engl. drain) und dem Substrat (aus [29]).

Abbildung 2 veranschaulicht dies am Beispiel eines simulierten MOS-FETs mit einer Kanallänge von $7 \mathrm{~nm}$ [29]. Es ist deutlich zu erkennen, dass die diskrete, zufällige Verteilung von Dotieratomen zu starken Fluktuationen des elektrostatischen Potentials führen kann, was letztendlich bei identisch hergestellten Transistoren individuell unterschiedliche Charakteristika in Bezug auf die Schaltspannung oder Leckströme bedingt [30]. Bei der Untersuchung dieser Effekte ist man derzeit vor allem auf Simulationen angewiesen, da sich die bislang verfügbaren experimentellen Methoden nicht dazu eignen, um die spezifische atomare Struktur eines Bauelements mit seinen elektronischen Eigenschaften in Verbindung zu bringen [29, 31]. Um auch in den kommenden Jahren eine kontinuierliche Miniaturisierung gewährleisten zu können, ist es daher von großem Interesse, das Zusammenspiel zwischen der präzisen atomaren Struktur und den lokalen elektronischen Eigenschaften eines Schottky-Kontakts experimentell detailliert untersuchen zu können.

Neben der konventionellen Halbleiterelektronik, die ausschließlich auf der Manipulation von elektrischen Ladungen beruht, gewinnt die Spintronik, in der zusätzlich der Spin-Freiheitsgrad der Ladungsträger ausgenutzt wird, immer mehr an Bedeutung [32, 33]. Einer der ersten Ansätze für ein solches halbleiterbasiertes Spintronik Bauelement geht auf Datta und Das zurück [34]. Bei diesem in Abbildung 3 dargestellten Spin-FET bestehen die Kontakte aus ferromagnetischen Materialien. Die Funktionalität beruht unter anderem darauf, spinpolarisierte Ladungsträger aus einem der Kontakte, der als Quelle dient, in das unmagnetische Halbleitermaterial zu injizieren und die resultierende SpinPolarisation am anderen Kontakt zu detektieren. 


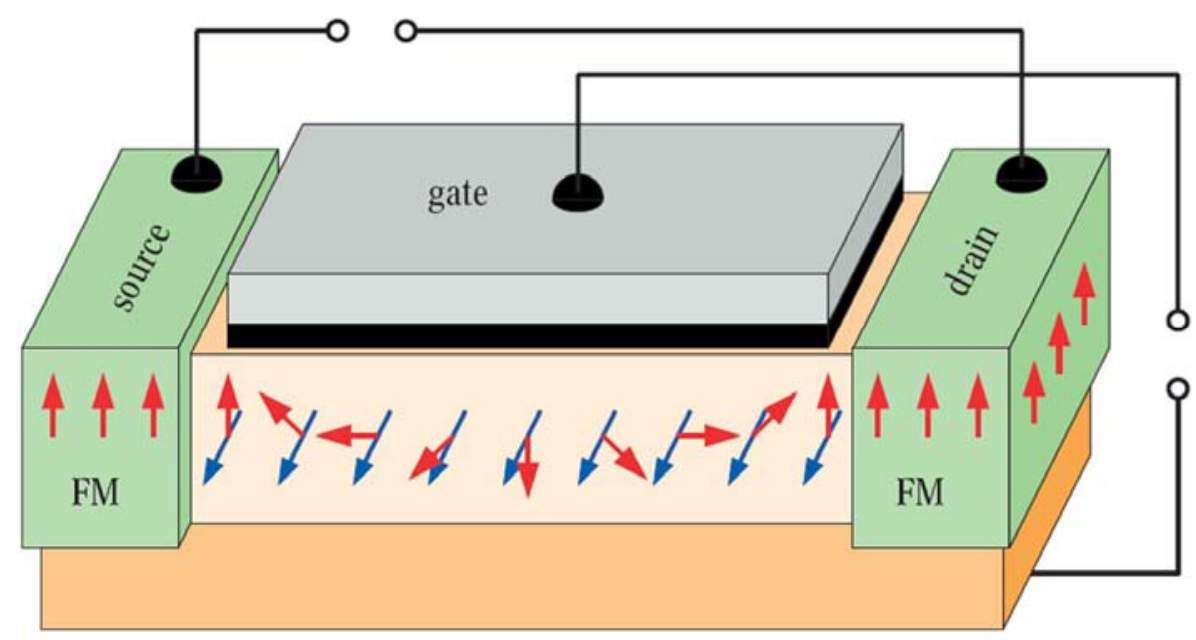

Abbildung 3: Schematische Darstellung eines Spin-FET nach Datta und Das [35]. Der linke ferromagnetische Kontakt injiziert spinpolarisierte Ladungsträger (rote Pfeile) in den Halbleiter, die unter dem Einfluss eines effektiven Magnetfeldes (blaue Pfeile), das über die Spannung an der Steuerelektrode (engl. gate) kontrolliert wird, zu der ebenfalls ferromagnetischen Senke präzidieren. Durch den Transistor fließt ein Strom, wenn die Spins der Ladungsträger am rechten Kontakt parallel zu seiner Magnetisierung ausgerichtet sind.

Eine Möglichkeit zur Realisierung eines Spin-FET besteht in der Verwendung von Kontakten aus ferromagnetischen Metallen. Sie bieten gegenüber ferromagnetischen Halbleitern den Vorteil von hohen Curie-Temperaturen, geringen Koerzitiv-Feldern und einer weit fortgeschrittenen technologischen Entwicklung. In verschiedenen Arbeiten wurde gezeigt, dass eine effektive Injektion durch diffusiven Spin-Transport aufgrund der stark unterschiedlichen elektrischen Leitfähigkeiten von Metall und Halbleiter allerdings nur im Fall eines nahezu 100 \% Spin-polarisierten Kontakts möglich ist [36, 37]. Eine Lösung dieses so genannten „conductivity mismatch“ Problems stellt ein zusätzlicher Spin-abhängiger Grenzflächenwiderstand dar, der z.B. über eine Tunnelbarriere realisiert werden kann [38]. Da eine Schottky-Diode bereits eine natürliche Tunnelbarriere aufweist, wurde in den vergangenen Jahren ein großer Aufwand betrieben, um ein epitaktisches Ferromagnet-Halbleiter System mit einer defektfreien, abrupten Grenzfläche zu entwickeln. In diesem Zusammenhang wurde insbesondere das nahezu gitterangepasste System Fe-GaAs von verschiedenen Gruppen sowohl theoretisch als auch experimentell intensiv untersucht [39-49]. Eine komplette Unterdrückung von Interdiffusionsprozessen an der Grenzfläche, durch die die bislang erreichbaren Spin-Injektionseffizienzen entscheidend reduziert werden, konnte bislang noch nicht erreicht werden [50-53].

Im Zentrum der vorliegenden Arbeit stand daher zum einen die Optimierung des epitaktischen Wachstums von dünnen Fe-Filmen auf GaAs\{110\} Substraten, wobei sowohl im Hinblick auf die Untersuchung der Schottky-Barrierenformierung als auch im Hinblick auf zukünftige Spintronik Bauelemente 
das Hauptaugenmerk auf der Unterdrückung von Reaktionen an der Metall-Halbleiter Grenzfläche lag. Zum anderen war es das Ziel, auf der Basis der Raster-Tunnel-Mikroskopie und -Spektroskopie einen experimentellen Zugang zu erarbeiten, der erstmals die Untersuchung des Zusammenspiels zwischen den atomaren strukturellen und elektronischen Eigenschaften eines Schottky-Kontakts ermöglicht.

Die Arbeit gliedert sich wie folgt:

In Kapitel 1 wird zunächst auf die wesentlichen Eigenschaften von Metall-Halbleiter Kontakten eingegangen, bevor im weiteren Verlauf des Kapitels die Grundzüge von unterschiedlichen theoretischen Modellen der Schottky-Barrierenformierung diskutiert werden.

Nachdem in Kapitel 2 die Messmethode der Raster-Tunnel-Mikroskopie (engl. scanning tunneling microscopy, STM) vorgestellt wurde, die im Zentrum dieser Arbeit steht, wird in Kapitel 3 am Beispiel der GaAs\{110\} Spaltflächen auf die Besonderheiten und Vorzüge von STM Untersuchungen in Querschnittsgeometrie (XSTM) eingegangen.

Das im Rahmen dieser Arbeit entwickelte zweistufige Verfahren zur Präparation von idealen FeGaAs $\{110\}$ Schottky-Kontakten wird in Kapitel 4 im Detail beschrieben.

In Kapitel 5 wird die Charakterisierung der so hergestellten Fe-Filme in Bezug auf ihre strukturellen und magnetischen Eigenschaften diskutiert. Die Untersuchungen umfassen die Charakterisierung der Filmmorphologie mit dem STM, die strukturelle Analyse über die Beugung niederenergetischer Elektronen (engl. low energy electron diffraction, LEED) und die Messung des magneto-optischen KerrEffekts (MOKE). Es wird gezeigt, dass im Gegensatz zu konventionell gewachsenen Fe-GaAs $\{110\}$ Schottky-Kontakten verschiedene Charakteristika beobachtet werden, die insbesondere im Zusammenhang mit einer homogenen, abrupten Grenzfläche erwartet werden (siehe auch [54]).

Um darüber hinausgehende, detaillierte Informationen über die atomaren strukturellen Eigenschaften des Bereichs der RLZ bis hin zur Metall-Halbleiter Grenzfläche zu erhalten, wurden STM Untersuchungen in Querschnittsgeometrie durchgeführt. In Kapitel 6 wird gezeigt, dass dieser neue Zugang zur Untersuchung von Metall-Halbleiter Kontakten, der in dieser Arbeitsgruppe entwickelt und optimiert wurde, zum ersten Mal die Möglichkeit bietet, die lokale atomare Struktur eines SchottkyKontakts zu bestimmen. Anhand von atomar aufgelösten XSTM Topografien des Grenzflächenbereichs wird unter anderem bestätigt, dass es durch das verwendete zweistufige Präparationsverfahren gelungen ist, einen idealen Fe-GaAs\{110\} Schottky-Kontakt zu wachsen.

Neben der topografischen Untersuchung wurde das Fe-GaAs $\{110\}$ System mit Methoden der RasterTunnel-Spektroskopie (engl. scanning tunneling spectroscopy, STS) in Querschnittsgeometrie untersucht. Diese in Kapitel 7 diskutierten Messungen erlauben es, die atomaren strukturellen Eigenschaften eines Schottky-Kontakts erstmals mit den lokalen elektronischen Eigenschaften zu verknüpfen. Im Zusammenhang mit 3D-Simulationen der spitzeninduzierten Bandverbiegung wird demonstriert, dass 
die STS Daten zudem neben der quantitativen Bestimmung der SBH auch die Untersuchung der Potentiallandschaft unter dem Einfluss der diskreten Verteilung von Dotieratomen erlauben. Des Weiteren wird am Ende des Kapitels darauf eingegangen, wie unter anderem anhand der experimentell bestimmten Charakteristika von Bandlückenzuständen im Bereich des Metall-Halbleiter Übergangs auf den Mechanismus der Schottky-Barrierenformierung geschlossen werden kann, der im Fall des idealen Fe-GaAs $\{110\}$ Schottky-Kontakts dominiert.

Die Arbeit schließt mit einer Zusammenfassung der experimentellen Ergebnisse. 


\section{Inhaltsverzeichnis}

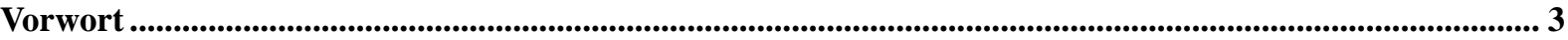

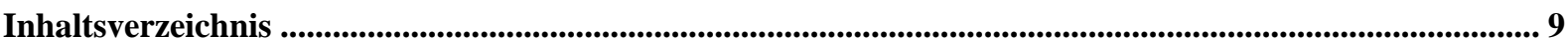

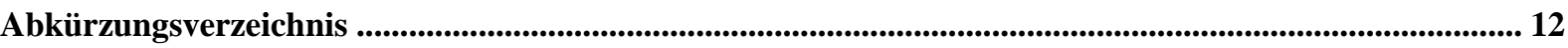

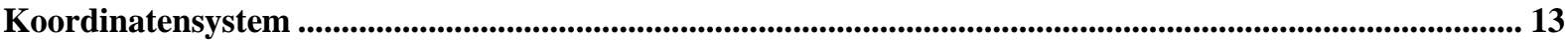

1 Der Metall-Halbleiter Kontakt - vom Spitzengleichrichter zum Spin-Injektor ..................................... 14

$1.1 \quad$ Der ideale Metall-Halbleiter Kontakt.................................................................................. 14

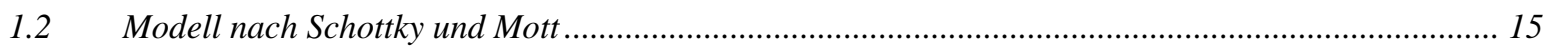

„1.3 „Fermi-Level Pinning “ durch Bandlückenzustände ................................................................... 18

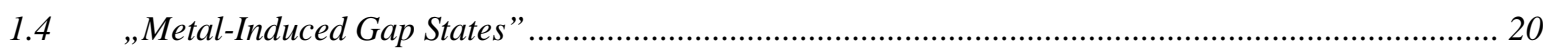

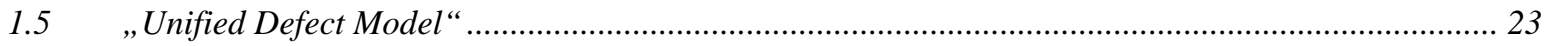

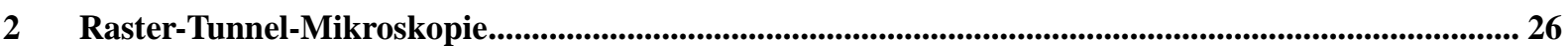

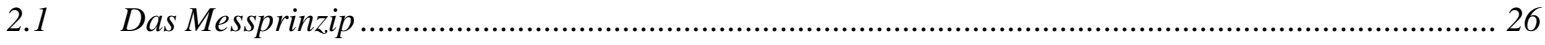

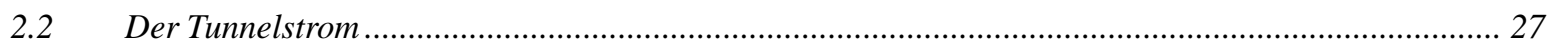

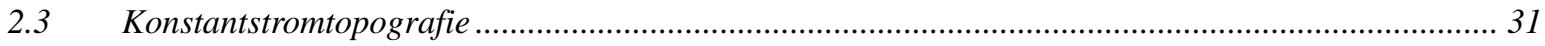

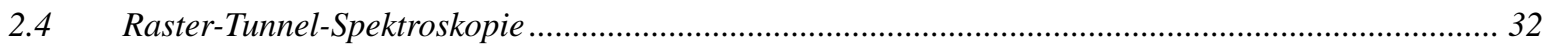

2.5 Spektroskopie der lokalen effektiven Barrierenhöhe............................................................... 33

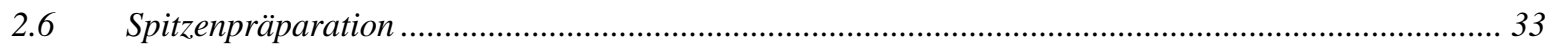

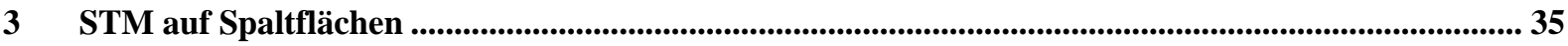

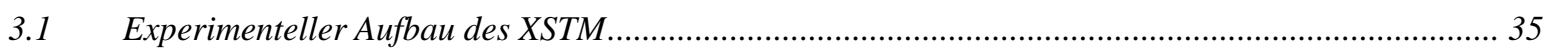

3.2 Atomare und elektronische Eigenschaften der GaAs\{110\} Spaltflächen ........................................ 37

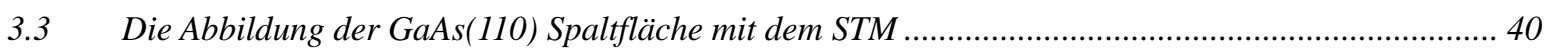

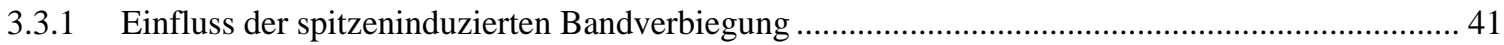

3.3.2 Simulation des Tunnelstroms bei STM Messungen auf GaAs\{110\} Spaltflächen...................... 45

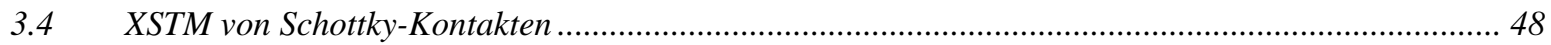

4 Präparation von idealen Schottky-Kontakten zur Untersuchung mit dem XSTM ..............................51 
4.1 Die GaAs\{110\} Spaltflächen als Substrat für ideale Schottky-Kontakte ........................................ 51

4.2 Vorbereitung des GaAs Substrats für die UHV Präparation ........................................................ 53

4.3 UHV Präparation des Schottky-Kontakts auf der GaAs(110) Spaltfläche bei tiefen Temperaturen ... 56

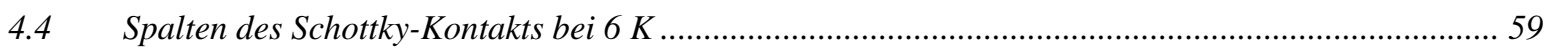

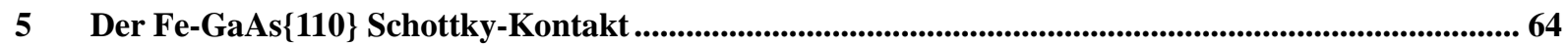

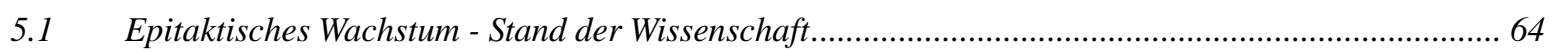

5.2 Fe-GaAs(110) Schottky-Kontakte durch Tieftemperatur-Deposition und anschließendes Annealen:

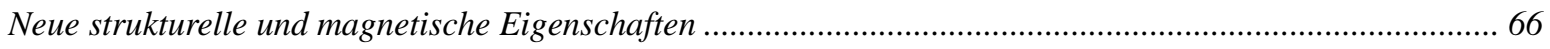

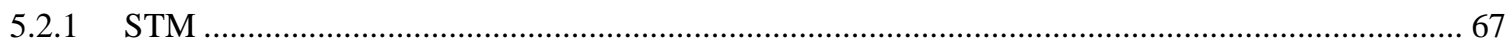

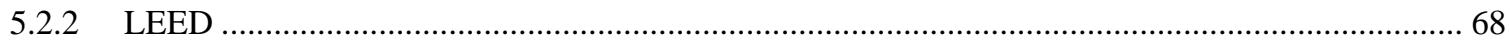

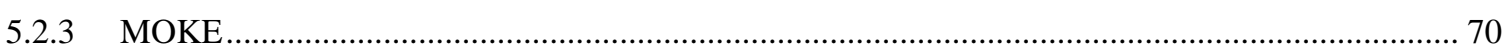

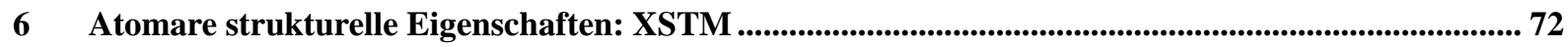

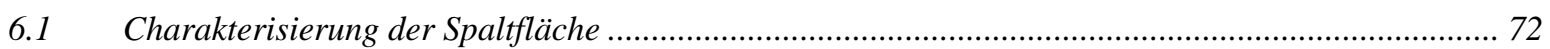

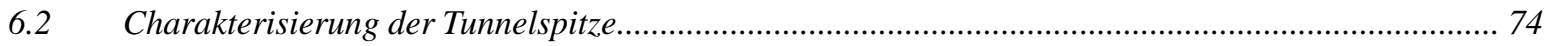

6.3 Charakterisierung der lokalen Tunnelbedingungen in der Raumladungszone ................................... 75

6.4 Strukturelle Eigenschaften des Fe-GaAs(110) Schottky-Kontakts ............................................... 81

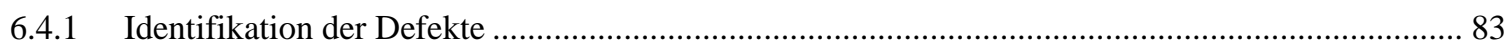

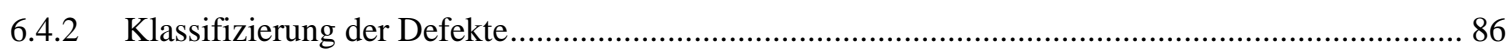

6.4.3 Atomare Struktur des Fe-GaAs(110) Schottky-Kontakts .................................................... 87

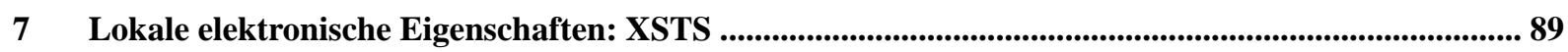

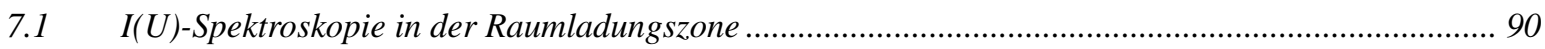

7.1.1 Einfluss des elektrostatischen Potentials in der RLZ: 1D-Simulation von I(U)-Kennlinien ........... 92

7.1.2 Das TIBB in der Raumladungszone des Schottky-Kontakts: 3D-Simulation der elektrostatischen

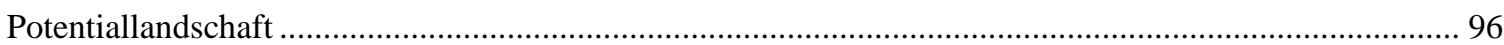

7.1.3 Die Oberflächenbandverbiegung bei positiven Bias-Spannungen als Verbindung zwischen

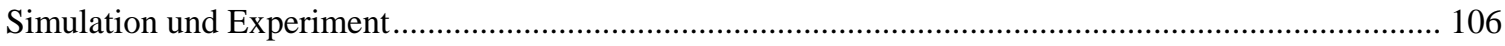

7.1.4 Auswirkungen des TIBB auf die I(U)-Spektroskopie in der Raumladungszone des SchottkyKontakts

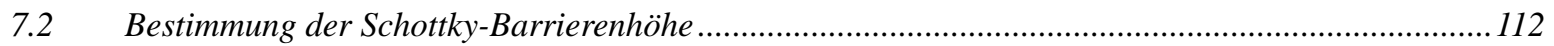

7.2.1 Variation des lokalen elektrostatischen Potentials in der Raumladungszone ................................119

7.3 I(U)-Spektroskopie im Bereich der Metall-Halbleiter Grenzfläche ............................................ 124 
Anhang A: FEM Simulation des TIBB bei der XSTM Untersuchung von Schottky-Kontakten .. 131

Literaturverzeichnis

Publikationen..

Präsentationen. 145

Danksagung ...

Lebenslauf 


\section{Abkürzungsverzeichnis}

\begin{tabular}{|c|c|c|}
\hline bcc & - & base centered cubic \\
\hline BEEM & - & Ballistische-Elektronen-Emissions-Mikroskopie \\
\hline DFT & - & Dichte-Funktional-Theorie \\
\hline FEM & - & Finite Elemente Methode \\
\hline FET & - & Feld-Effekt Transistor \\
\hline FLP & - & Fermi-level pinning \\
\hline GaAs & - & Galliumarsenid \\
\hline IPMA & - & in-plane magnetic anisotropy \\
\hline MBE & - & Molekularstrahl-Epitaxie \\
\hline MIGS & - & metal-induced gap states \\
\hline ML & - & Monolage \\
\hline MOKE & - & Magneto-optischer Kerr-Effekt \\
\hline LEED & - & low-energy electron diffraction \\
\hline $\mathrm{LT}$ & - & low temperature \\
\hline PES & - & Photoemissions-Spektroskopie \\
\hline REM & - & Raster-Elektronen-Mikroskopie \\
\hline RLZ & - & Raumladungszone \\
\hline RT & - & Raumtemperatur \\
\hline SBB & - & surface band-bending \\
\hline SBH & - & Schottky-Barrierenhöhe \\
\hline STM/STS & - & scanning tunneling microscopy/spectroscopy \\
\hline TEM & - & Transmissions-Elektronen-Mikroskopie \\
\hline TIBB & - & tip-induced band-bending \\
\hline (A)UDM & - & (advanced) unified defect model \\
\hline UHV & - & Ultra-Hoch-Vakuum \\
\hline
\end{tabular}


WKB - $\quad$ Wentzel-Kramers-Brillouin

\section{Koordinatensystem}

In dieser Arbeit wurde durchgängig das in Abbildung 4 dargestellte kartesische Koordinatensystem verwendet.

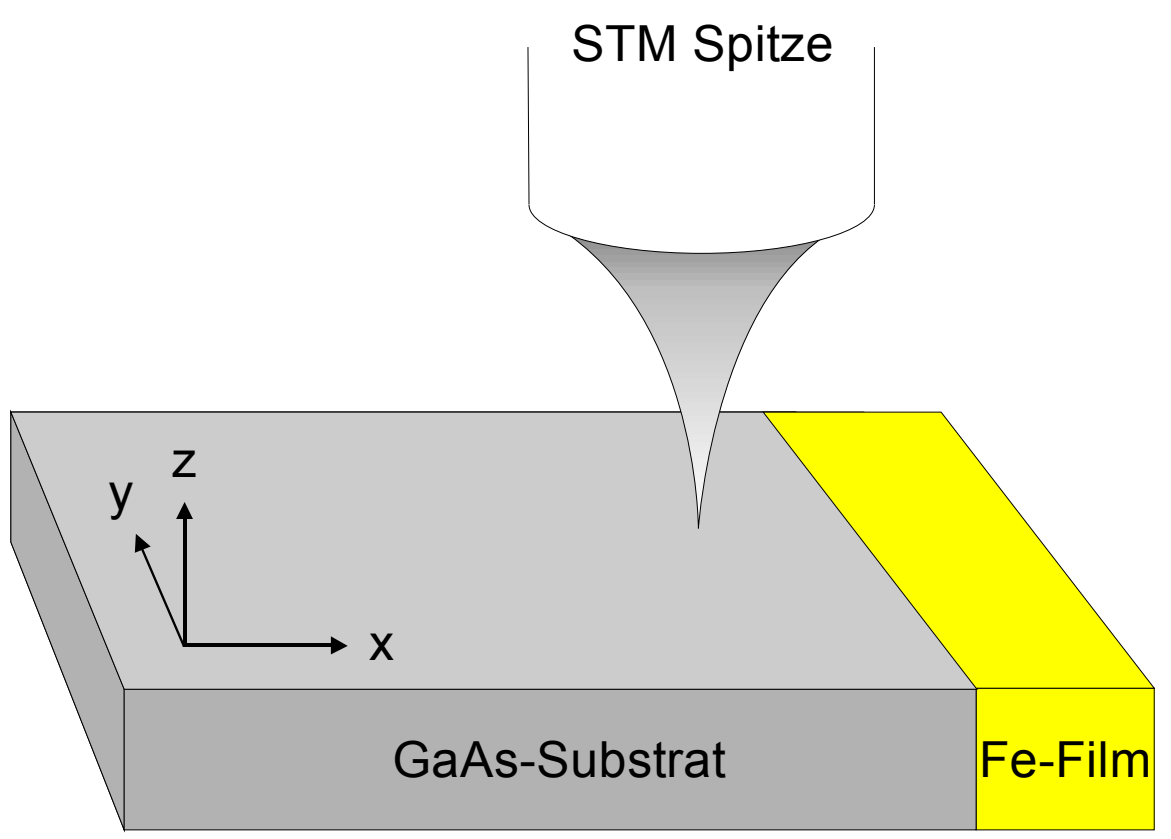

Abbildung 4: Schematische Darstellung eines Metall-Halbleiter Kontakts bei einer STM Untersuchung in Querschnittsgeometrie.

Die x-Achse bezeichnet generell die Richtung senkrecht zur Metall-Halbleiter Grenzfläche, die yAchse ist parallel zur Grenzfläche ausgerichtet. Die Richtung senkrecht zu der Oberfläche, die mit dem STM untersucht wird, entspricht der z-Achse. In Schemaskizzen wird das GaAs Substrat grau und der Metallfilm gelb dargestellt. 


\section{Der Metall-Halbleiter Kontakt - vom Spitzengleich- richter zum Spin-I njektor}

Bereits im Jahr 1874 entdeckte Ferdinand Braun, dass ein Punktkontakt zwischen einem Metall und einem Halbleiter zu einem stromrichtungsabhängigen Widerstand führt [1]. Die unipolare Leitfähigkeit dieser als Spitzengleichrichter bezeichneten ersten Bauelemente der Halbleiterelektronik konnte 1938 von Walter Schottky erstmals auf der Basis von mikroskopischen Bandstrukturmodellen erklärt werden [3]. Es stellte sich allerdings schnell heraus, dass viele experimentelle Beobachtungen an den in der Folge als Schottky-Kontakt bezeichneten Metall-Halbleiter Kontakten nicht durch dieses grundlegende Modell allein erklärt werden können. Seit dem wurden eine Reihe verschiedener Ansätze entwickelt, um diese Abweichungen zu erklären. Mittlerweile ist klar, dass die Ausbildung eines Schottky-Kontakts mit seiner charakteristischen effektiven Schottky-Barrierenhöhe durch unterschiedliche Mechanismen bestimmt werden kann. Es wird allerdings noch heute kontrovers diskutiert, welcher dieser Mechanismen die Physik des Schottky-Kontakts dominiert.

Das Kapitel beginnt zunächst mit der Einführung des idealen Metall-Halbleiter Kontakts, der ein zentrales Element dieser Arbeit darstellt. Im weiteren Verlauf des Kapitels werden beginnend mit der Schottky-Mott-Theorie einige der Modelle vorgestellt, die zur Beschreibung von Metall-GaAs $\{110\}$ Systemen diskutiert werden. Die Darstellung bezieht sich dabei jeweils auf das für diese Arbeit relevante Regime geschlossener Metallfilme auf einem n-dotierten GaAs $\{110\}$ Substrat. Für einen detaillierten und umfassenden Überblick über die Entwicklungen auf dem Gebiet der Schottky-Physik stehen eine Reihe von verschiedenen Quellen zur Verfügung [20, 55, 56].

\subsection{Der ideale Metall-Halbleiter Kontakt}

Historisch gesehen besaßen ideale Schottky-Kontakte lange Zeit nur theoretische Bedeutung. Da es sich um die einfachste Struktur eines Metall-Halbleiter Kontakts handelt, bei dem beide homogenen kristallinen Teilsysteme durch eine planare, abrupte Grenzfläche getrennt werden, dienten Sie als Modellsysteme in verschiedenen Theorien zur Beschreibung der Physik von Metall-Halbleiter Kontakten [3-8]. Erst mit der Einführung von UHV Präparationstechniken ist es möglich geworden, ideale Schottky-Kontakte auch experimentell zu realisieren [20]. Im Gegensatz zu den herkömmlichen, sehr komplexen Schottky-Kontakten besitzen sie einen wohl definierten Grenzflächenbereich. Ideale Schottky-Kontakte eigenen sich daher optimal, um den Zusammenhang zwischen den strukturellen und elektronischen Eigenschaften eines Metall-Halbleiter Kontakts zu untersuchen. Durch ihre einfa- 
che Grundstruktur ist es in der Regel möglich, die atomare Struktur im Bereich der homogenen Grenzfläche z.B. durch Transmissions-Elektronen-Mikroskopie (TEM) experimentell zu bestimmen [16]. Diese strukturellen Eigenschaften können anschließend dazu verwendet werden, um die Charakteristika der Schottky-Barriere theoretisch im Rahmen verschiedener Modelle zu berechnen. Durch einen Vergleich mit den experimentell bestimmten elektronischen Eigenschaften kann schließlich die Relevanz der Modelle in Bezug auf die Ausbildung der Schottky-Barriere gezielt getestet werden. Ideale Schottky-Kontakte haben daher vor allem einen hohen wissenschaftlichen Stellenwert und können zu einem besseren Verständnis der grundlegenden physikalischen Zusammenhänge bei Metall-Halbleiter Kontakten beitragen. In aktuellen halbleiterbasierten elektronischen und optoelektronischen Bauteilen sind sie allerdings bislang kaum von Bedeutung. Durch die Entwicklung von Spintronik Bauelementen auf der Basis von Ferromagnet-Halbleiter Hybridstrukturen könnte sich dies grundlegend ändern, da die Funktionalität dieser Systeme sehr stark von der strukturellen Ordnung an der Grenzfläche abhängig ist [50,57]. Ideale Schottky-Kontakte können daher entscheidend zur Verbesserung der Effizienz von Spintronik Bauelementen beitragen.

\subsection{Modell nach Schottky und Mott}

Unabhängig voneinander haben Schottky und Mott nahezu zeitgleich eine grundlegende Theorie erarbeitet, um die bis dahin unverstandene gleichrichtende Wirkung von Metall-Halbleiter Kontakten auf der Basis eines einheitlichen Modells zu erklären [3, 4]. Die Grundidee der nach ihnen benannten Schottky-Mott-Theorie soll in Abbildung 5 durch ein Gedankenexperiment verdeutlicht werden. In dem Modell wird angenommen, dass Metall und Halbleiter ideale, planare Oberflächen besitzen, die ein einem Abstand $d$ parallel zueinander ausgerichtet sind. Beide Systeme befinden sich im thermischen Gleichgewicht und sind an ihren Rückseiten durch einen Draht elektrisch leitfähig miteinander verbunden, so dass die Fermi-Niveaus $E_{F}{ }^{M}$ und $E_{F}{ }^{S C}$ angeglichen sind (Abbildung 5 (a)). ${ }^{1}$

\footnotetext{
${ }^{1}$ Die in dieser Arbeit verwendeten Indizes leiten sich in der Regel aus den jeweiligen englischen Bezeichnungen ab (z.B. SC für semiconductor etc.).
} 
(a)

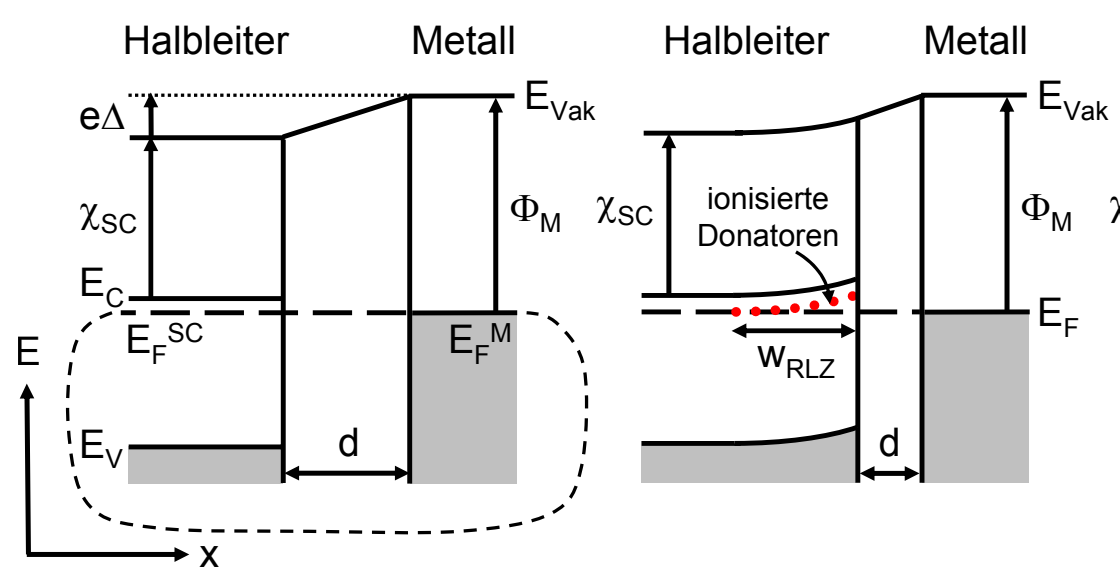

(c)

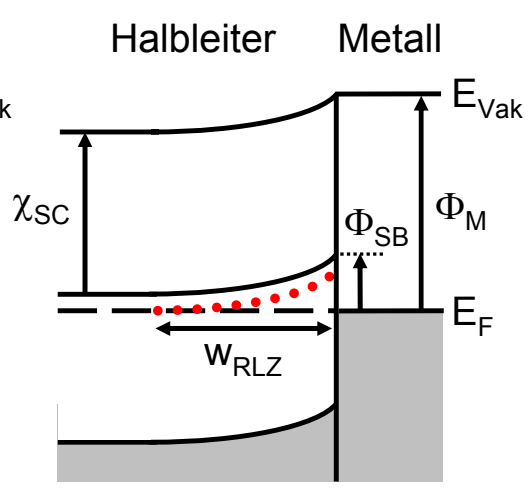

Abbildung 5: Die Schottky-Mott-Relation in einem Gedankenexperiment. (a) Metall und Halbleiter sind weit voneinander entfernt. Beide Systeme sind durch einen Draht elektrisch leitfähig miteinander verbunden, so dass die Fermi-Niveaus $E_{F}{ }^{M}$ und $E_{F}{ }^{S C}$ auf beiden Seiten angeglichen sind. Die Energien von Valenz- und Leitungsbandkante $\left(E_{V}\right.$ und $\left.E_{C}\right)$ des n-dotierten Halbleiters sind eingezeichnet. Die Energiebereiche, in denen die Zustände besetzt sind, sind jeweils grau dargestellt. (b) Für kleine Abstände d kommt es im Halbleiter zu der Ausbildung einer Raumladungszone mit der Ausdehnung $w_{R L Z}$, in der das elektrische Feld durch ionisierte Donatoren abgeschirmt wird. (c) Metall und Halbleiter befinden sich in direktem Kontakt und der gesamte Potentialabfall findet in der Raumladungszone des Halbleiters statt.

Wenn die Austrittsarbeiten von Metall $\left(\phi_{M}\right)$ und Halbleiter $\left(\phi_{S C}=\chi_{S C}+\left(E_{C}-E_{F}{ }^{S C}\right)\right)$ nicht identisch sind, was in der Regel der Fall ist, existiert zwischen den beiden Systemen ein Kontaktpotential mit $e \Delta=\phi_{M}-\phi_{S C} \cdot{ }^{2}$ Bei dem Modell von Schottky und Mott wird davon ausgegangen, dass der Halbleiter keinen Oberflächenzustand besitzt, der das elektrische Feld bei geringer werdendem Abstand $d$ effektiv an der Oberfläche abschirmen könnte (Abbildung 5 (b)).In dem hier gezeigten Beispiel $\left(\phi_{M}>\phi_{S C}\right)$ werden die Bänder im Bereich des Halbleiters durch das lokale elektrostatische Potential zu höheren Energien hin verschoben und es entsteht eine Raumladungszone (RLZ), in der positiv ge-

\footnotetext{
${ }^{2}$ Die Austrittsarbeit $\phi$ bzw. die Elektronenaffinität $\chi$ ist definiert als die Energie, die erforderlich ist, um ein Elektron von dem Fermi-Niveau $E_{F}$ bzw. dem Leitungsbandminimum $E_{C}$ gerade außerhalb des Festkörpers zu platzieren (,gerade außerhalb“ bezeichnet hierbei eine Distanz von typischer Weise $100 \mathrm{~nm}$ - $10 \mu \mathrm{m}$ zur Oberfläche) [20, 143]. Das entsprechende Energieniveau wird als Vakuumniveau $E_{V a k}$ bezeichnet. Es handelt sich hierbei nicht um das Energieniveau $E_{V a k, \infty}$ eines ruhenden Elektrons an einem unendlich weit entfernten Punkt.
} 
ladene ionisierte Donatoren das Feld abschirmen. Je geringer der Abstand $d$ zwischen den beiden Systemen wird, desto größer wird die RLZ. Für $d \rightarrow 0$, d.h. Metall und Halbleiter befinden sich in direktem Kontakt, findet der gesamte Potentialabfall in der RLZ des Halbleiters statt (Abbildung 5 (c)). Es herrscht Ladungsneutralität, so dass die Gesamtladung der ionisierten Donatoren in der RLZ des Halbleiters $\left(Q_{S C}\right)$ durch eine gleich große Ladung umgekehrten Vorzeichens an der Oberfläche des Metallfilms $\left(Q_{M}\right)$ ausgeglichen wird:

$$
Q_{S C}+Q_{M}=0
$$

Es wird angenommen, dass sich $\phi_{M}$ und $\chi_{S C}$ nicht ändern, so dass die SBH $\phi_{S B}^{0}$ ohne eine externe Vorspannung ausschließlich durch die Differenz der Austrittsarbeiten von Metall und Halbleiter bestimmt wird (Schottky-Mott-Relation): ${ }^{3}$

$$
\phi_{S B}^{0}=\phi_{M}-\chi_{S C}
$$

Die Schottky-Mott-Relation kann daher als die erste Theorie betrachtet werden, die eine konkrete Vorhersage über die SBH eines Systems trifft. Gemäß (1-2) sollte die Barrierenhöhe linear mit der Austrittsarbeit des Metalls variieren. Sofern die Austrittsarbeit des Metalls kleiner als die Elektronenaffinität des Halbleiters ist, sollte der Schottky-Kontakt kein Diodenverhalten zeigen.

In Übereinstimmung mit der Vorstellung von Schottky und Mott konnte abgesehen von wenigen Ausnahmen tatsächlich experimentell verifiziert werden, dass Metalle mit höherer Austrittsarbeit systematisch zu höheren SBHs führen als solche mit geringeren Austrittsarbeiten [20]. Die experimentell beobachtete Abhängigkeit der SBH von der Austrittsarbeit des Metalls ist allerdings in den meisten Fällen wesentlich schwächer, als von der Schottky-Mott-Theorie vorhergesagt [58].

Abgesehen von den Aussagen über die SBH eines Systems ist es Schottky in seiner Theorie erstmals gelungen, die Ursache der spannungsabhängigen Potentialbarriere für Majoritätsladungsträger durch die Verschiebung der Bänder in der RLZ des Halbleiters korrekt zu beschreiben. Er hat vermutet, dass es zur Ausbildung einer Verarmungszone kommt, in der der Verlauf des elektrostatischen Potentials $V(\vec{r})$ die Poisson-Gleichung erfüllen muss:

$$
\nabla^{2} V(\vec{r})=-\frac{\rho(\vec{r})}{\varepsilon \varepsilon_{0}}
$$

\footnotetext{
${ }^{3}$ In dieser Arbeit wird ausschließlich die Situation ohne externe Vorspannung betrachtet, so dass der Index 0 im weiteren Verlauf der Arbeit nicht mehr explizit angegeben wird.
} 
$\rho(\vec{r})$ ist die lokale Ladungsdichte, $\varepsilon_{0}$ ist die Permittivität des Vakuums und $\varepsilon$ ist die Dielektrizitätskonstante des Halbleiters. Unter der Annahme, dass die Ladungsdichte entlang der Achse $x$ senkrecht zur idealen Metall-Halbleiter Grenzfläche ausschließlich durch die ionisierten Donatoren der Dichte $N_{D}$ hervorgerufen wird und durch eine Stufenfunktion beschrieben werden kann (Besetzung der elektronischen Zustände bei $T=0$ ), d.h.

$$
\rho(x)= \begin{cases}e N_{D}, & 0<x<w_{R L Z} \\ 0, & x>w_{R L Z}\end{cases}
$$

ergibt sich für die Weite der RLZ

$$
w_{R L Z}=\sqrt{\frac{2 \varepsilon \varepsilon_{0}}{e^{2} N_{D}}\left(\phi_{S B}-\left(E_{C}-E_{F}\right)\right)}
$$

wobei $\phi_{S B}-\left(E_{C}-E_{F}\right)=\phi_{S B B}$ die Bandverbiegung an der Oberfläche bzw. Grenzfläche angibt. Der Verlauf der Leitungsbandkante $E_{C}(x)$ ergibt sich als Lösung der Poisson-Gleichung (Rigid-BandModell):

$$
E_{C}(x)= \begin{cases}\phi_{S B B}\left(1-\frac{x}{w_{R L Z}}\right)^{2}+\text { const., } & 0<x<w_{R L Z} \\ \text { const., } & x>w_{R L Z}\end{cases}
$$

Historisch gesehen hat das Schottky-Mott-Modell wesentlich zum grundlegenden Verständnis der physikalischen Mechanismen beigetragen, die die Eigenschaften von Schottky-Kontakten beeinflussen. Bei der Vorhersage der SBH eines Systems kann der Theorie allerdings nur wenig Relevanz eingeräumt werden.

\section{3 „Fermi-Level Pinning“ durch Bandlückenzustände}

Schon kurz nach der Einführung der Schottky-Mott-Theorie wurde festgestellt, dass dieses Modell zu einfach war, um die Vielzahl der experimentellen Erkenntnisse im Zusammenhang mit MetallHalbleiter Kontakten erklären zu können. Aufbauend auf den Überlegungen von Schottky und Mott wurden daher in der zweiten Hälfte des 20. Jahrhunderts verschiedene Theorien entwickelt, die sich vor allem mit der Frage beschäftigen, was die charakteristische SBH bestimmt, wenn Metall und Halbleiter in Kontakt gebracht werden. 
1947 schlug Bardeen ein Modell von, in dem Oberflächenzustände, die sich energetisch in der fundamentalen Bandlücke des Halbleiters befinden, eine zentrale Rolle spielen [5]. Wenn der Halbleiter mit dem Metall in Kontakt gebracht wird kommt es zu einem Ladungstransfer in diese Bandlückenzustände (engl. gap states) und es bildet sich ein zusätzlicher Dipol $\Delta_{G S}$ im Bereich der Grenzfläche aus, der die Differenz zwischen der Austrittsarbeit des Metalls und der Elektronenaffinität des Halbleiters zum Teil ausgleicht. Abbildung 6 verdeutlicht diesen Zusammenhang.

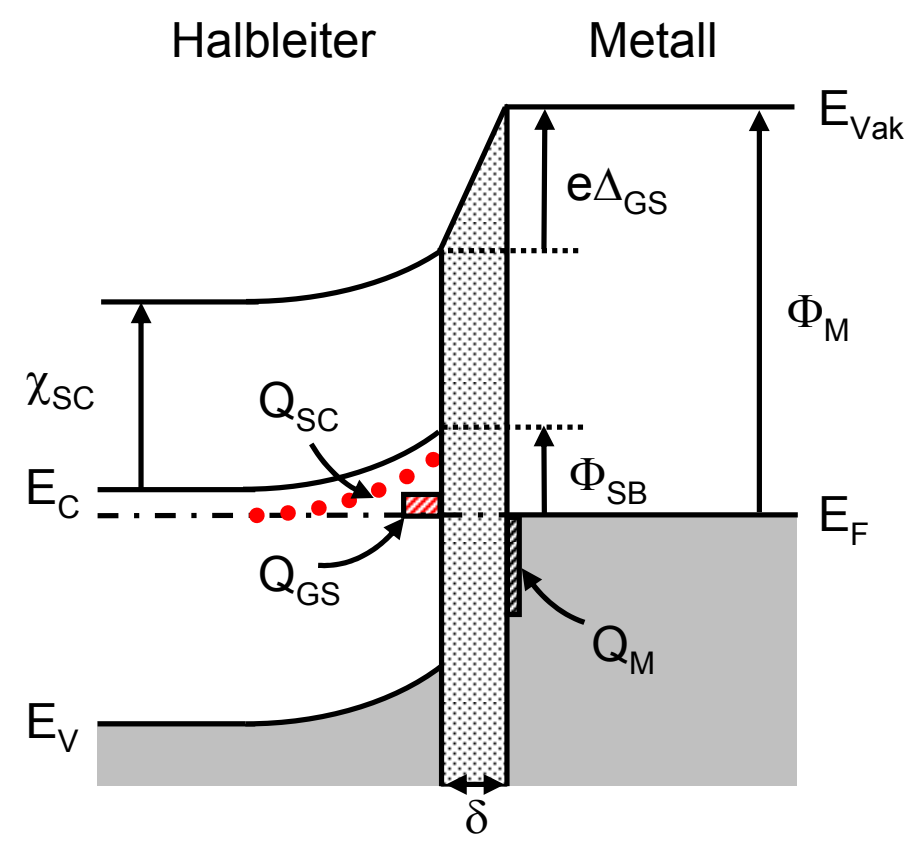

Abbildung 6: Schematische Darstellung eines Schottky-Kontakts mit Bandlückenzuständen. Metall und Halbleiter sind durch eine Grenzflächenschicht der Dicke $\delta$ (einige Ångström dick) voneinander getrennt. Durch die Ladungen $Q_{G S}$ in den Bandlückenzuständen kommt es zu einem zusätzlichen Potentialabfall in der Grenzflächenschicht.

Da ein zusätzlicher Grenzflächendipol, der zu einer Abweichung der SBH von der Schottky-MottRelation (1-2) führt, nur existieren kann, wenn die Bandlückenzustände räumlich von der MetallHalbleiter Grenzfläche getrennt sind, wird angenommen, dass sich eine nur wenige Ångström dicke, dielektrische Grenzflächenschicht zwischen Metall und Halbleiter befindet. Über dieser Schicht fällt die elektrostatische Energie um den Betrag $e \cdot \Delta_{G S}$ ab, um den die Schottky-Mott-Relation (1-2) erweitert werden muss:

$$
\phi_{S B}^{0}=\phi_{M}-\chi_{S C}-e \cdot \Delta_{G S}
$$

Auch die Relation (1-1) muss entsprechend um die Ladungen $Q_{G S}$, die sich in den Bandlückenzuständen befinden, ergänzt werden: 


$$
Q_{S C}+Q_{M}+Q_{G S}=0
$$

Bardeen hat gezeigt, dass bereits eine Dichte der Bandlückenzustände von weniger als $10^{13} / \mathrm{eV} \mathrm{cm}{ }^{2}$ dazu führen kann, dass verschiedene Metalle auf demselben Halbleitersubstrat nahezu identische SBHs besitzen. In diesem Zusammenhang wird häufig der Begriff „Fermi-level pinning“ (FLP) verwendet, da das Fermi-Niveau unabhängig von der Austrittsarbeit des Metalls in einem engen Energiebereich innerhalb der Bandlücke des Halbleiters gehalten wird [20, 24, 56].

Das von Bardeen diskutierte FLP durch Bandlückenzustände ist seit dem der zentrale Punkt vieler Theorien, in denen verschiedene physikalische Ursachen für das Auftreten von Grenzflächenzuständen diskutiert werden.

\section{4 „Metal-I nduced Gap States"}

In der Arbeit von Bardeen wurde das FLP durch Bandlückenzustände erklärt, die im Sinne von intrinsischen Oberflächenzuständen des ungestörten Halbleitersubstrats eingeführt wurden [5]. Der Einfluss des Metallfilms auf die elektronische Struktur des Schottky-Kontakts wurde erst 1965 von Heine explizit in die Betrachtung der Bandlückenzustände einbezogen [6]. Er konnte zeigen, dass der Metallfilm im Bereich der Grenzfläche ein Kontinuum von Zuständen in der Bandlücke des Halbleiters verursacht. Durch diese metallinduzierten Zustände (engl. metal-induced gap states, MIGS) mit einer Dichte von $>3,5 \cdot 10^{14} / \mathrm{eV} \mathrm{cm}^{2}$ besitzt der Halbleiter lokal metallische Eigenschaften, die das experimentell beobachtete FLP erklären können. Strukturelle Veränderungen der Subsysteme im Bereich der Grenzfläche ebenso wie starke chemische Bindungen zwischen Metall und Halbleiter spielen in dem MIGS Modell keine Rolle. Die Überlegungen von Heine wurden in den folgenden Jahren in verschiedenen anderen Arbeiten aufgegriffen und weiter ausgearbeitet [7, 8, 19, 59]. Die wesentlichen Aspekte des MIGS Modells werden im Folgenden kurz zusammengefasst.

An einer freien Metalloberfläche fallen die Wellenfunktionen der Elektronen exponentiell ins Vakuum ab. Grenzt das Metall an einen Halbleiter, so dringen die Wellenfunktionen in den Halbleiter ein. Heine konnte zeigen, dass dies im Energiebereich der fundamentalen Bandlücke des Halbleiters zu einem Kontinuum von Zuständen führt, die im Metall den Charakter von Volumenzuständen besitzen und im Halbleiter exponentiell abklingen [6]. Eine detaillierte Berechnung der elektronischen Struktur im Bereich der Grenzfläche von verschiedenen idealen Metall-Halbleiter Kontakten wurde von Louie et al. präsentiert [7]. Zur numerischen Berechnung wurde eine selbstkonsistente PseudopotentialMethode verwendet, wobei das Metall als Jellium modelliert wurde. Abbildung 7 zeigt die berechnete Zustandsdichte in verschiedenen Bereichen eines idealen Al-GaAs(110) Schottky-Kontakts. 


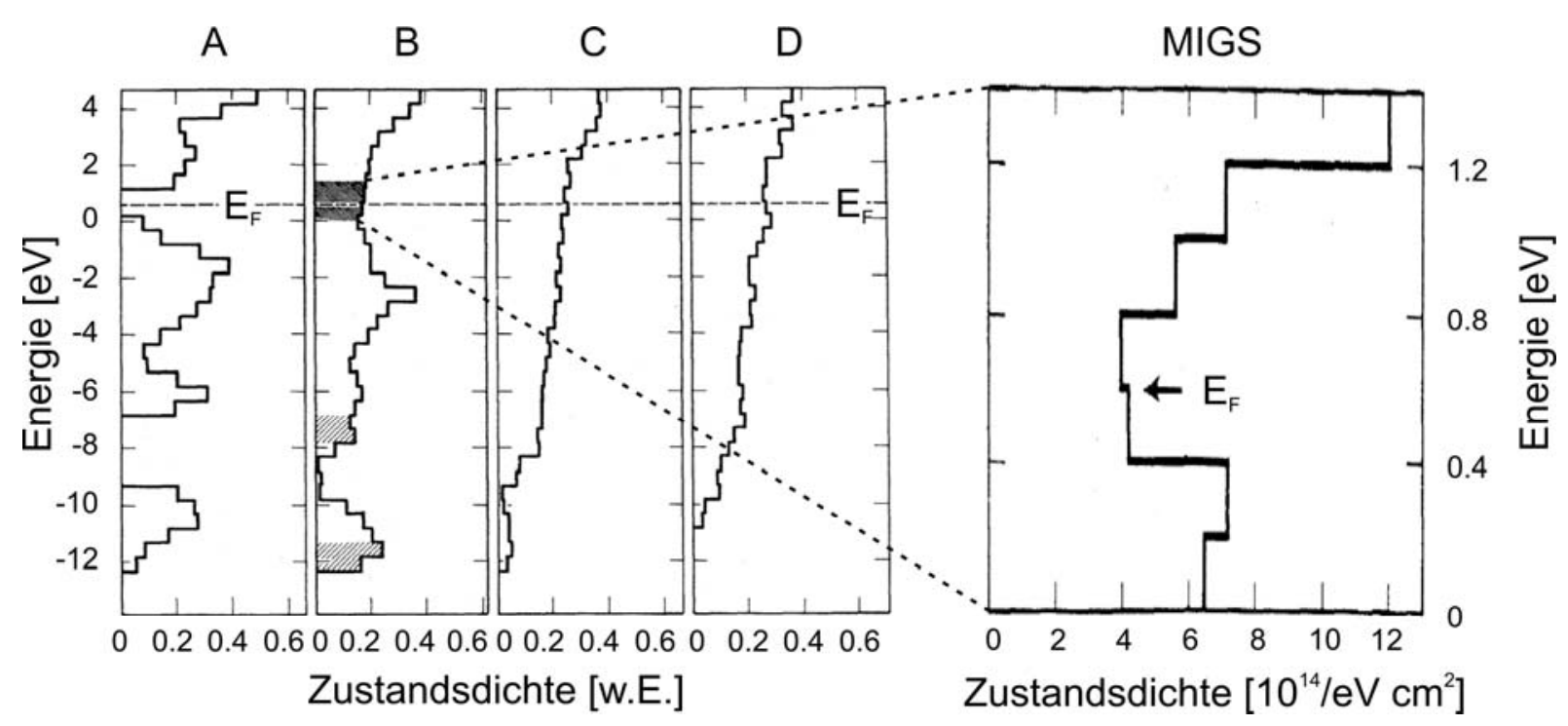

Abbildung 7: (aus [7]) Lokale Zustandsdichte eines Al-GaAs(110) Schottky-Kontakts. Die gezeigten Regionen befinden sich im Zentrum des Halbleiterbereichs (A), an der Grenze zum Metallfilm (B), im Metallfilm an der Grenzfläche zum Halbleiter (C) und in der Mitte des Metallfilms (D). Der Bereich der fundamentalen Bandlücke in Region B ist auf der rechten Seite vergrößert dargestellt.

Region A zeigt die Situation im Zentrum des simulierten Halbleiterbereichs, Region B entspricht der atomaren Lage des Halbleiters direkt an der Grenzfläche, Region C stellt den daran angrenzenden Bereich des Metalls dar und Region D zeigt die Situation in der Mitte des Metalls. Die MetallHalbleiter Grenzfläche verläuft entsprechend zwischen Region B und C. Im Gegensatz zum Metallfilm, in dessen lokaler Zustandsdichte bis zur Grenzfläche hin kaum Veränderungen zu beobachten sind, wird die Zustandsdichte des Halbleiters durch den benachbarten Metallfilm stark beeinflusst. Anstelle der fundamentalen Bandlücke, die in Region A deutlich zu erkennen ist, zeigen die Rechnungen direkt an der Grenzfläche ein Kontinuum von Zuständen. Der entsprechende Energiebereich ist rechts in Abbildung 7 vergrößert dargestellt. Die Zustandsdichte der MIGS nimmt von den Bandkanten zur Mitte der Bandlücke hin ab, sie bleibt aber im gesamten Bereich der Bandlücke größer als $3 \cdot 10^{14} / \mathrm{eV} \mathrm{cm}^{2}$ und ist von daher in der Lage, dass Fermi-Niveau effektiv in der Bandlücke zu stabilisieren [5]. Aus den Rechnungen ergibt sich darüber hinaus die Energie, bei der das FLP stattfindet, so dass es Louie et al. erstmals gelungen ist, die SBH von verschiedenen Metall-Halbleiter Kontakten selbstkonsistent zu berechen. Aus der Lage des Fermi-Niveaus, das rechts in Abbildung 7 eingezeichnet ist, ergibt sich für den Al-GaAs(110) Schottky-Kontakt eine SBH von $\phi_{S B}=0,8 \pm 0,2 \mathrm{eV}$ [7].

Das energieabhängige Abklingverhalten der MIGS wird durch die komplexe Bandstruktur des Halbleiters bestimmt, da es sich wie auch bei Oberflächenzuständen um Bloch-Zustände des Halbleiters mit komplexem Wellenvektor handelt. Der Zusammenhang zwischen der räumlichen Ausdehnung der MIGS und der komplexen Bandstruktur des Halbleiters ist in Abbildung 8 skizziert. 
(a) Komplexe Bandstruktur

(b) Ausdehnung der MIGS

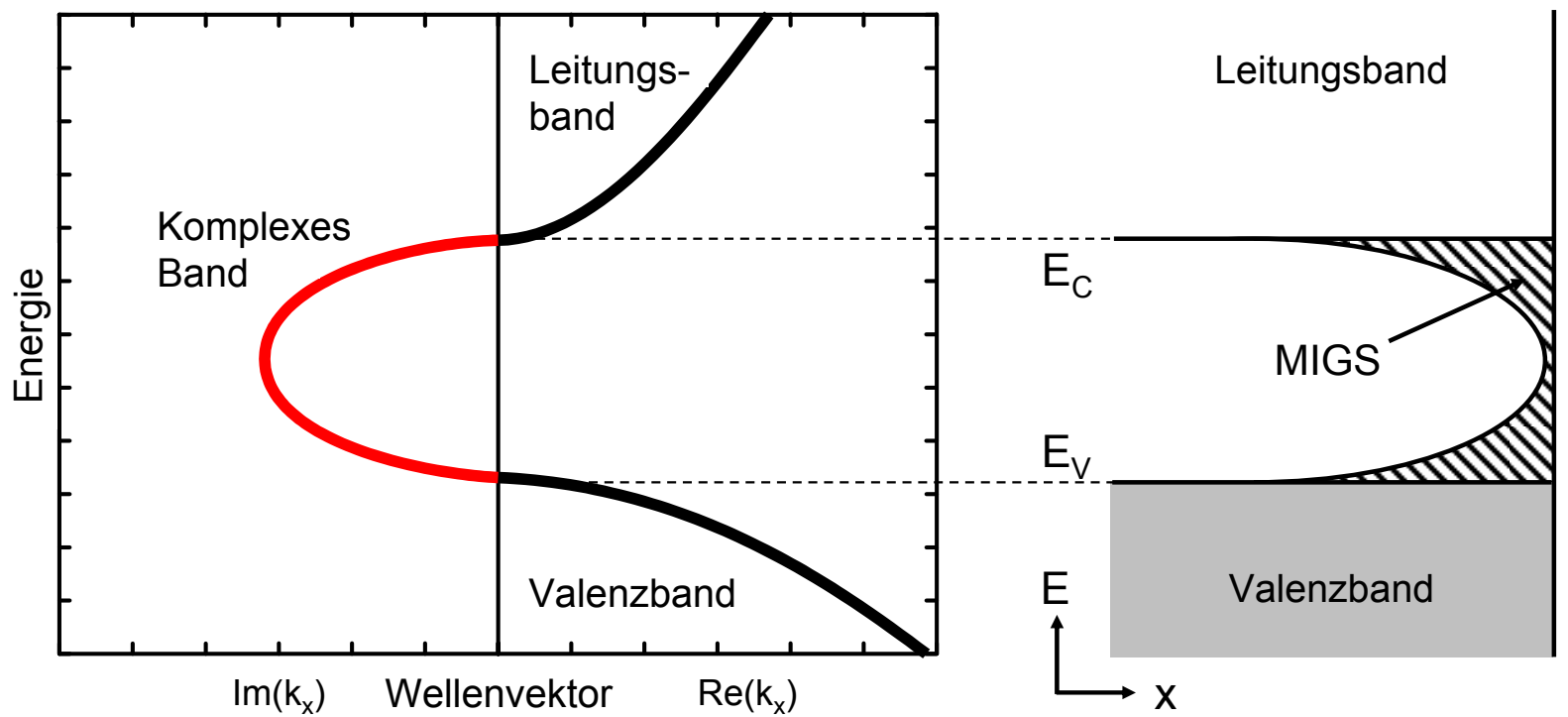

Abbildung 8: (a) Schematische Darstellung der komplexen Bandstruktur eines Halbleiters mit einem Leitungs- und Valenzband, die durch ein komplexes Band mit imaginären Wellenvektoren verbunden werden. Die Abklingkonstante $\kappa$ ist im Zentrum der Bandlücke maximal und nimmt zu den Bandkanten hin ab. (b) Skizze des energieabhängigen Abklingverhaltens der MIGS. Die räumliche Ausdehnung der MIGS nimmt zu den Bandkanten hin zu.

Abbildung 8 (a) zeigt, dass Valenz- und Leitungsband in der komplexen Ebene durch ein komplexes Band verbunden werden. Die Zustände im Energiebereich der Bandlücke besitzen komplexe Wellenvektoren, so dass die Wellenfunktionen exponentiell abklingen. Die Abklingkonstante $\kappa$ nimmt von den Bandkanten zum Zentrum der Bandlücke hin zu. Dies führt dazu, dass die MIGS im Zentrum der Bandlücke am schnellsten abklingen und zu den Bandkanten hin immer weiter in den Bereich des Halbleiters eindringen (Abbildung 8 (b)). Im Fall eines GaAs\{110\} Substrats beträgt die über den Bereich der Bandlücke gemittelte Abklingkonstante $\kappa$ etwa $3 \AA$ [7, 8]. Der Einfluss des Metallfilms auf die Zustandsdichte im Halbleiter besitzt daher nur eine sehr kurze Reichweite, so dass die Zustandsdichte bereits in einem Abstand von nur drei Monolagen im Wesentlichen wieder der im Volumen des Halbleiters entspricht.

Auch der Charakter der MIGS wird durch die Bandstruktur des Halbleiters bestimmt. Sie haben entweder überwiegend Leitungsband- oder überwiegend Valenzbandcharakter, je nachdem ob sie sich in der Bandlücke bei höheren oder niedrigeren Energien befinden [60]. Die Energie, bei der der Übergang stattfindet, befindet sich bei Halbleitern wie GaAs etwa in der Mitte der Bandlücke und kann über die komplexe Volumenbandstruktur berechnet werden [8]. Tersoff hat gezeigt, dass diese intrinsische Übergangsenergie bei dem FLP durch MIGS eine entscheidende Rolle spielt. Da im Halbleiter Ladungsneutralität herrscht, wenn alle Zustände mit dominierendem Valenzbandcharakter besetzt und alle mit überwiegend Leitungsbandcharakter unbesetzt sind, entspricht die Übergangsenergie dem 
Ladungsneutralitätsniveau (engl. charge neutrality level, CNL) der MIGS. Zustände mit Energien unterhalb des CNL können als donatorartig (neutral oder positiv geladen) beschrieben werden, wohingegen sich MIGS oberhalb des CNL akzeptorartig (neutral oder negativ geladen) verhalten. Befindet sich das Fermi-Niveau über bzw. unterhalb des CNL, so existiert im Halbleiter eine zusätzliche negative bzw. positive Ladung $Q_{G S}$ im Sinne von (1-8). Da die MIGS eine hohe Zustandsdichte mit einem kontinuierlichen und glatten Verlauf besitzen führt bereits eine geringfügige Verschiebung des FermiNiveaus relativ zum CNL zu einer hohen zusätzlichen Ladung $Q_{G S}$. Die Energie, bei der das FLP stattfindet, wird daher im MIGS Modell in erster Linie durch die Lage des intrinsischen CNL des Halbleiters bestimmt [8]. Bei den in dieser Arbeit verwendeten GaAs\{110\} Substraten befindet sich das berechnete CNL 0,50 eV über der Valenzbandkante [59].

Die darüber hinausgehende Abhängigkeit des FLP von dem Metallfilm wurde von Mönch untersucht [61]. Er konnte zeigen, dass die Differenz der Elektronegativitäten von Metall und Halbleiter bestimmt, wie viel Ladung in die MIGS transferiert wird. Die Energie, bei der das FLP stattfindet, liegt daher systematisch über, bei oder unter dem CNL, je nachdem ob das Metall eine kleinere, dieselbe oder eine größere Elektronegativität als der Halbleiter besitzt.

Insbesondere in dem in dieser Arbeit untersuchten Regime geschlossener Metallfilme auf GaAs $\{110\}$ Substraten konnte die Relevanz des MIGS Modells bereits in verschiedenen Arbeiten demonstriert werden [7, 8, 59, 61]. In Verbindung mit dem Konzept des CNL und unter Berücksichtigung der Elektronegativität des Metalls ist das MIGS Modell in der Lage, eine Vielzahl von experimentell bestimmten SBHs von Metall-GaAs\{110\} Systemen einheitlich zu erklären.

\section{5 „Unified Defect Model“}

Das „Unified Defect Model“ (UDM), das auf Arbeiten von Spicer et al. zurückgeht, wurde ursprünglich entwickelt, um eine Vielzahl von experimentellen Beobachtungen zu erklären, die unter anderem bei der Untersuchung von Metall-GaAs\{110\} Systemen gemacht wurden [9]:

- Aus der Photoemissions-Spektroskopie (PES) sowie theoretischen Berechnungen geht hervor, dass saubere GaAs\{110\} Oberflächen keinen Oberflächenzustand in der fundamentalen Bandlücke besitzen, der das FLP im Sinne von Bardeen erklären könnte.

- Abweichend von dem MIGS Modell, bei dem das FLP durch Grenzflächenzustände zwischen dem Halbleiter und einem geschlossenen Metallfilm erklärt wird, zeigen PES Messungen von verschiedenen Metall-GaAs\{110\} Systemen, dass es schon nach der Deposition von weit weniger als einer Monolage zu einem FLP kommt. 
- Die energetische Lage, bei der das FLP stattfindet, ist nahezu unabhängig von dem deponierten Metallfilm und folgt keinem chemischen Trend.

Motiviert durch diese verschiedenen experimentellen Beobachtungen schlussfolgern Spicer et al., dass die SBH nicht durch die Eigenschaften der deponierten Metallatome bestimmt werden kann. In dem UDM wird postuliert, dass während des Wachstumsprozesses in der Nähe der Grenzfläche generell sekundäre, für den Halbleiter typische Defekte erzeugt werden. Diese Defekte verursachten Defektzustände bei charakteristischen Energien in der fundamentalen Bandlücke des Halbleiters, in deren Nähe das FLP unabhängig von dem deponierten Metall stattfindet. Die entsprechende Situation ist in Abbildung 9 skizziert.

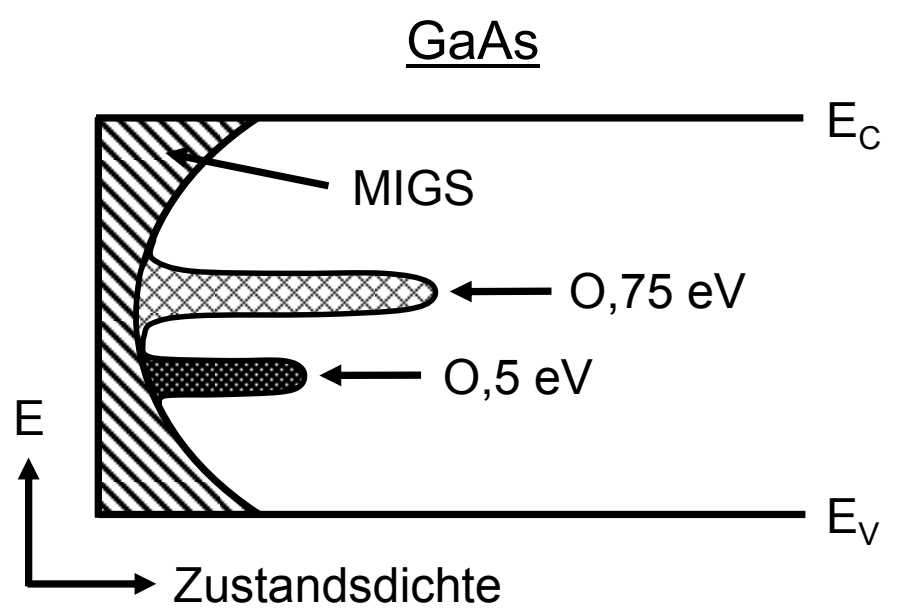

Abbildung 9: (nach [62]) Skizze der lokalen Zustandsdichte im GaAs Substrat an der MetallHalbleiter Grenzfläche. Dargestellt ist der Energiebereich der fundamentalen Bandlücke. Die akzeptor- bzw. donatorartigen Defektzustände befinden sich 0,75 eV bzw. 0,5 eV über der Valenzbandkante.

$\mathrm{Zu}$ der Zustandsdichte im Energiebereich der Bandlücke des Halbleiters tragen an der MetallHalbleiter Grenzfläche sowohl die Defektzustände als auch das Kontinuum der Grenzflächenzustände bei. Neben dem donatorartigen Defektzustand, der sich 0,5 eV über der Valenzbandkante befindet, existiert ein akzeptorartiger Defektzustand bei einer Energie von 0,75 eV über der Valenzbandkante. Im Fall von den in dieser Arbeit verwendeten n-dotierten GaAs\{110\} Substraten geht das UDM davon aus, dass das Fermi-Niveau stets durch den akzeptorartigen Defektzustand bei 0,75 eV stabilisiert wird, so das bei Raumtemperatur (RT) unabhängig von dem verwendeten Metall SBHs von etwa $0,7 \mathrm{eV}$ erwartet werden [62].

Es wird zunächst vermutet, dass atomare Fehlstellen für die Defektzustände verantwortlich sind, da angenommen wird, dass diese Defekte am häufigsten bei den untersuchten Substratoberflächen auftreten [9]. Im Fall von n-GaAs\{110\} Substraten gehen Spicer et al. davon aus, dass As-Fehlstellen den akzeptorartigen Defektzustand bei 0,75 eV verursachen. Bei p-GaAs\{110\} Substraten wird der dona- 
torartige Defektzustand bei 0,5 eV entsprechend mit Ga-Fehlstellen in Verbindung gebracht. Neuere Untersuchungen von GaAs\{110\} Oberflächen mit dem Raster-Tunnel-Mikroskop zeigen allerdings, dass entgegen der ursprünglichen Vermutung bei n-GaAs Substraten im Wesentlichen Ga-Fehlstellen auftreten und bei p-GaAs Substraten vor allem As-Fehlstellen beobachtet werden [63].

In einer Weiterentwicklung des UDMs, dem „Advanced Unified Defect Model“ (AUDM), identifizieren Spicer et al. den für das FLP verantwortlichen Defekt als ein substitutionell auf einem Ga-Gitterplatz eingebautes As-Atom ( $\mathrm{As}_{\mathrm{Ga}}$-Antisite) [10]. Bei diesem Defekt handelt es sich um ein Doppeldonator mit experimentell bestimmten Defektzuständen bei 0,52 eV und 0,77 eV über der Valenzbandkante [64]. Durch diesen Doppeldonator alleine kann das FLP bei n-dotierten Substraten allerdings nicht erklärt werden, so dass im Bereich der Grenzfläche ein zusätzlicher, kompensierender Akzeptor existieren muss. Es wird vermutet, dass es sich hierbei um $\mathrm{Ga}_{\mathrm{As}}$-Antisite Defekte handelt, deren Existenz allerdings bislang nicht nachgewiesen werden konnte [10].

Die Rolle der Defektkonzentration in der Nähe der Metall-Halbleiter Grenzfläche wurde von Zur et al. theoretisch für den Fall eines unendlich dicken, geschlossenen Metallfilms und für den Fall einer Submonolagenbedeckung untersucht [65]. Es wurde angenommen, dass die durch die Defekte hervorgerufenen Grenzflächenzustände im Wesentlichen im Bereich des Halbleiters lokalisiert sind und der Abstand zur Grenzfläche nur wenige Ångström beträgt. Aus den Berechnungen geht hervor, dass in dem in dieser Arbeit untersuchten Regime geschlossener Metallfilme eine Defektdichte von $10^{14} / \mathrm{cm}^{2}$ notwendig ist, um ein FLP zu verursachen.

Im Gegensatz zu dem MIGS Modell wird in dem UDM/AUDM davon ausgegangen, dass es während der Deposition des Metallfilms zu einer starken Wechselwirkung zwischen Metall und Halbleiter kommt, die zur Ausbildung einer hohen extrinsischen Defektdichte im Bereich des Halbleiters führt. ${ }^{4}$ Die Relevanz des UDM/AUDM konnte vor allem im Regime der Submonolagenbedeckungen sowie bei Schottky-Kontakten mit inhomogenen, defektreichen Grenzflächen gezeigt werden [10, 19, 66].

\footnotetext{
${ }^{4}$ Die generelle Präsenz der für den Halbleiter charakteristischen Defekte, die für das FLP verantwortlich gemacht werden, konnte allerdings bislang nicht nachgewiesen werden.
} 


\section{Raster-Tunnel-Mikroskopie}

Die Eigenschaften von Schottky-Kontakten wurden in den vergangenen Jahrzehnten intensiv experimentell untersucht. Bislang existiert allerdings keine allgemein anerkannte Theorie, die die gewonnenen Daten zufrieden stellend erklären kann, da in den meisten Studien wichtige Details über die lokale atomare und elektronische Struktur im Bereich der Grenzfläche nicht ausreichend bekannt sind. Das STM bietet die Möglichkeit, sowohl strukturelle als auch elektronische Eigenschaften eines Systems mit exzellenter räumlicher Auflösung zusammenhängend zu untersuchen. In Bezug auf die in Kapitel 1 diskutierten Modelle können mit dieser Messmethode wichtige Merkmale wie der Verlauf der lokalen Zustandsdichte im Energiebereich der fundamentalen Bandlücke oder die Existenz von charakteristischen Defekten bestimmt werden.

Zu Beginn des Kapitels wird kurz auf das Messprinzip des STMs eingegangen, das die zentrale experimentelle Methode dieser Arbeit darstellt. Der darauf folgende Abschnitt befasst sich zunächst mit der allgemeinen Beschreibung des Tunnelstroms, bevor Besonderheiten im Zusammenhang mit dem STM Experiment diskutiert werden. Für eine ausführliche theoretische Beschreibung des Tunneltransports bei dem STM wird auf die Arbeiten von J. Chen [67], R. Wiesendanger [68] und D. Bonell [69] verwiesen. Die verschiedenen in dieser Arbeit verwendeten Messmodi sowie wichtige Details der Spitzenpräparation werden am Ende des Kapitels dargestellt.

\subsection{Das Messprinzip}

Unter dem Begriff Raster-Sonden-Techniken wird eine Reihe von vergleichsweise jungen Messmethoden zusammengefasst, mit denen Strukturen bis hinunter auf die atomare Skala untersucht werden können. Sie alle beruhen auf demselben Arbeitsprinzip, dass auf Entwicklungen von Gerd Binnig und Heinrich Rohrer aus dem Jahr 1981 zurückgeht [70]:

- Eine möglichst mikroskopische Sonde wird soweit an die zu untersuchende Oberfläche der Probe angenähert, bis es zu einer lokalen, stark abstandsabhängigen Wechselwirkung kommt.

- Diese Wechselwirkung wird gemessen und dazu verwendet, um den Abstand zwischen Sonde und Probe zu kontrollieren.

- In einem Rasterverfahren wird die Sonde über die zu untersuchende Oberfläche bewegt. Mit Hilfe eines Regelkreises wird die Wechselwirkung auf einem bestimmten Wert konstant gehalten, der über den Setzpunkt definiert wird. 
Die Sonde des STMs ist in der Regel eine sehr scharfe, metallische Spitze. Als abstandsabhängige Wechselwirkung zwischen der Spitze und der ebenfalls leitfähigen Probe wird der Tunnelstrom $I_{T}$ verwendet, der durch die Vakuumbarriere zwischen den beiden Elektroden fließt, wenn eine externe Tunnelspannung $U_{\text {Bias }}$ angelegt wird. Diese Spannung in der Größenordnung von typischerweise einigen Volt, die auch als Probenspannung bzw. Bias-Spannung bezeichnet wird, ist definitionsgemäß auf die Probe bezogen, während sich die Spitze auf einem geerdeten Potential befindet. Bedingt durch das exponentielle Abfallen der Zustände in der Vakuumbarriere ist auch die Größe des Tunnelstroms exponentiell von dem Abstand $d$ zwischen Spitze und Probe abhängig, was die extrem hohe räumliche Auflösung des STMs ermöglicht:

$$
I \propto \exp (-2 \kappa d)
$$

$$
\kappa=\frac{\sqrt{2 m \phi_{B}}}{\hbar}
$$

Die Abklingkonstante $\kappa$ hängt in dieser vereinfachten Betrachtung ausschließlich von der Barrierenhöhe $\phi_{B}$ zwischen Spitze und Probe ab. Um Tunnelströme im Bereich von mehreren hundert Picoampère (pA) erreichen zu können, muss die STM Spitze während der Messung bis auf typischer Weise 6-9 Å an die Probenoberfläche angenähert werden [71-73].

\subsection{Der Tunnelstrom}

Bei diesen geringen Spitze-Probe Abständen von weniger als $10 \AA$ ist es nicht mehr möglich, den Tunneltransport im Rahmen von Tunnelprozessen durch eine klassische Kastenpotentialbarriere zu beschreiben. Spitze (engl. tip) und Probe (engl. sample) befinden sind bereits so nah aneinander, dass die Coulomb-Potentiale der letzten Spitzenatome mit denen der Oberflächenatome überlappen (Abbildung 10 (b)).

Das Standardmodell zur theoretischen Beschreibung des Tunneltransports beim STM basiert auf der 1961 von Bardeen entwickelten Transfer-Hamiltonoperator Näherung, die ursprünglich zur Beschreibung von makroskopischen, planaren Tunnelkontakten entwickelt wurde [74]. 


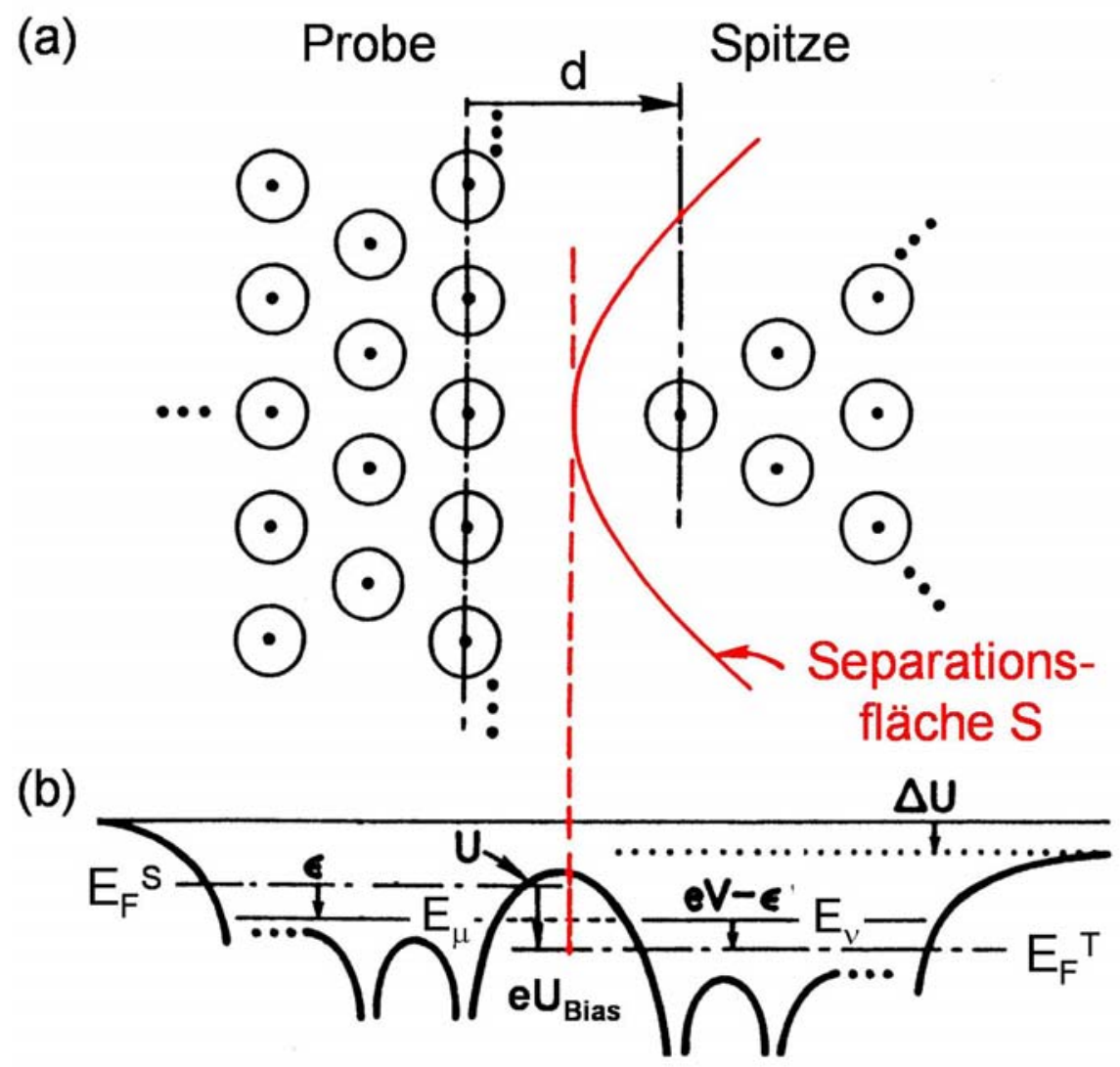

Abbildung 10: (a) Schematische Darstellung des Tunnelkontakts beim STM. Das Gesamtsystem wird durch die Separationsfläche $S$ in die Subsysteme von Spitze und Probe aufgetrennt. (b) Potentiallandschaft des Gesamtsystems. Die Differenz zwischen den Fermi-Energien $E_{F}{ }^{S}$ der Probe und $E_{F}{ }^{T}$ der Spitze entspricht der angelegten Bias-Spannung.

Nach Bardeen wird der Transfer eines Teilchens aus einem Zustand $\psi_{\mu}$ der Probe in einen Zustand $\chi_{\nu}$ der Spitze durch das Transfermatrixelement $M_{\mu \nu}$ beschrieben:

$$
M_{\mu \nu}=-\frac{\hbar^{2}}{2 m} \int_{S}\left(\chi_{\nu}^{*} \nabla \psi_{\mu}-\psi_{\mu} \nabla \chi_{\nu}^{*}\right) \cdot d \vec{S}
$$

Die Integration wird über eine Separationsfläche $S$ zwischen Spitze und Probe ausgeführt (siehe Abbildung 10 (a)). Bei einer gegebenen Probenspannung $U_{\text {Bias }}$ kann der Tunnelstrom $I_{T}$ durch den folgenden Ausdruck berechnet werden:

$$
I_{T}=\frac{2 \pi e}{\hbar} \sum_{\mu, v} f\left(E_{\mu}\right)\left[1-f\left(E_{v}+e U_{\text {Bias }}\right)\right]\left|M_{\mu \nu}\right|^{2} \delta\left(E_{\mu}-E_{\nu}\right)
$$


Der Tunnelstrom ergibt sich aus der Summe der Übergänge zwischen den Zuständen $\psi_{\mu}$ der Probe und den Zuständen $\chi_{v}$ der Spitze, wobei Tunnelprozesse nur stattfinden können, wenn zum einen $E_{\mu}=E_{v}$ gilt ${ }^{5}$ und zum anderen einer der Zustände gemäß der Fermi-Verteilungsfunktion $f(E)$ besetzt und der andere unbesetzt ist. Die Wahrscheinlichkeit eines Übergangs entspricht dabei dem Betragsquadrat des Transfermatrixelements $\left|M_{\mu \nu}\right|^{2}$.

Das Modell von Bardeen wurde 1983 von Tersoff und Hamann erweitert, um auch die bei STM Experimenten übliche Punktkontaktgeometrie analytisch beschreiben zu können [75, 76]. Unter der Voraussetzung, dass die Spitze durch eine sphärisch symmetrische s-Wellenfunktion beschrieben werden kann und dass nur Zustände nahe der Fermi-Energie der Probe an Tunnelprozessen beteiligt sind (kleine Bias-Spannungen und tiefe Temperaturen), gilt für den Tunnelstrom $I_{T}$ :

$$
I_{T} \propto e U_{\text {Bias }} \operatorname{DOS}_{T}\left(E_{F}^{T}\right) \sum_{\mu}\left(\left|\psi_{\mu}\left(\vec{r}_{T}\right)\right|^{2}\right) \delta\left(E_{\mu}-E_{F}{ }^{s}\right)
$$

$D_{T}(E)$ steht in diesem Ausdruck für die Zustandsdichte der Spitze (engl. density of states, DOS). Die Summe entspricht der Zustandsdichte der Probe bei der Fermi-Energie am Ort $\vec{r}_{T}=(x, y, z=d)$. Es handelt sich hierbei um den Schwerpunkt der s-Wellenfunktion der Spitze, der sich aus der lateralen Position $(x, y)$ und dem Tunnelabstand $d$ ergibt. Anhand von (2-5) wird deutlich, dass der Tunnelstrom neben der Abhängigkeit von der Zustandsdichte der Spitze und der Bias-Spannung im Rahmen der oben genannten Näherungen direkt proportional zur Zustandsdichte der Probe am Ort $\vec{r}_{T}$ ist. Diese wird im Allgemeinen als lokale Zustandsdichte (engl. local density of states, LDOS) bezeichnet:

$$
\operatorname{LDOS}\left(\vec{r}_{T}, E\right) \equiv \sum_{\mu}\left(\left|\psi_{\mu}\left(\vec{r}_{T}\right)\right|^{2}\right) \delta\left(E_{\mu}-E\right)
$$

Bei der Untersuchung von Metallen werden die Bedingungen des Tersoff-Hamann Modells häufig erfüllt, da nur selten große Bias-Spannungen verwendet werden. Bei anderen Probensystemen wie z.B. Halbleitern müssen allerdings im Allgemeinen Probenspannungen angelegt werden, die typischerweise in einem Bereich zwischen $\left|U_{\text {Bias }}\right| \approx 1 V-3 V$ liegen. Die Energieabhängigkeit des Transfermatrixelements kann unter diesen Bedingungen nicht mehr vernachlässigt werden. Um den Tunnelstrom beschreiben zu können, haben Hamers et. al. ein vereinfachtes Modell vorgeschlagen, in dem die

5 elastisches Tunneln 
Matrixelemente in Form eines energieabhängigen Transmissionskoeffizienten $T\left(E, e U_{B i a s}, d\right)$ behandelt werden [77].

$$
I_{T} \propto \quad \int_{E_{F}}^{E_{F}+e U_{\text {Bias }}} L D O S\left(\vec{r}_{T}, E\right) \operatorname{DOS}_{T}\left(E-e U_{\text {Bias }}\right) \cdot T\left(E, e U_{\text {Bias }}, d\right) d E
$$

Im Gegensatz zu dem Tersoff-Hamann Model bezeichnet $\vec{r}_{T}$ in diesem Fall die Position $(x, y, z=0)$ an der Probenoberfläche. Zu dem Tunnelstrom tragen sämtliche Zustände in dem Energieintervall zwischen $E_{F}$ und $E_{F}+e U_{\text {Bias }}$ bei. Der Transmissionskoeffizient eines Elektrons mit der Energie $E$ bei einer Probenspannung $U_{\text {Bias }}$ wird im Rahmen der Wentzel-Kramers-Brillouin Näherung (WKB) berechnet:

$$
T\left(E, e U_{\text {Bias }}, d\right)=\exp \left(-\frac{2 d}{\hbar} \sqrt{2 m\left(\frac{\phi_{T}+\phi_{S}}{2}+\frac{e U_{\text {Bias }}}{2}-E\right)}\right)
$$

Der Transmissionskoeffizient berücksichtigt sowohl das energieabhängige Abklingverhalten der Zustände in der Vakuumbarriere als auch die Form der Vakuumbarriere, die von der angelegten BiasSpannung abhängig ist.

In die angegebenen Ausdrücke für den Tunnelstrom (Gl. (2-5) und (2-8)) gehen sowohl die LDOS der Probe als auch die Zustandsdichte der Spitze gleichermaßen ein. Um eine Fokussierung auf die Eigenschaften der Probe zu erreichen, wird die Spitze in der Regel als eine nicht näher bekannte Sonde betrachtet, deren Zustandsdichte in dem relevanten Energiebereich als konstant angenommen wird. ${ }^{6}$ Unter dieser Voraussetzung gilt für den Tunnelstrom $I_{T}$ :

- Bei kleinen Probenspannungen ist der Tunnelstrom direkt proportional zur integralen LDOS der Probe

- Bei größeren Probenspannungen, wie sie in der Regel bei STM Messungen von Halbleitern verwendet werden, hängt der Tunnelstrom nicht mehr einfach von der integralen LDOS der Probe ab, da Zustände bei höheren Energien aufgrund des Transmissionskoeffizienten $T\left(E, e U_{B i a s}, d\right)$ stärker zum Tunnelstrom beitragen.

${ }^{6}$ Ob diese Näherung gerechtfertigt ist, muss im Einzelfall experimentell überprüft werden, da es ansonsten vor allem bei Tunnelspitzen mit stark modulierter Zustandsdichte zu Fehlinterpretationen der Messdaten kommen kann. 


\subsection{Konstantstromtopografie}

Bei der Konstantstromtopografie handelt es sich um den grundlegenden Messmodus der RasterTunnel-Mikroskopie. Während die STM Spitze bei einer festen Bias-Spannung (Setzspannung) zeilenweise über die zu untersuchende Oberfläche bewegt wird, wird die z-Position der Spitze durch einen Regelkreis immer so eingestellt, dass der Tunnelstrom auf einem festgelegten Wert (Setzstrom) konstant gehalten wird. An diskreten Punkten $(x, y)$ wird die z-Position der Spitze aufgenommen, die beispielsweise als Funktion des Ortes farbkodiert aufgetragen werden kann. Die auf diese Art entstehenden $z(x, y)$-Karten werden im Folgenden als Topografie bezeichnet. Da in diesem Messmodus der Tunnelstrom konstant gehalten wird, der den Ausführungen in Kapitel 2.2 entsprechend von den lokalen elektronischen Eigenschaften der Probe anhängig ist, handelt es sich bei einer STM Topografie stets um eine Überlagerung der Oberflächenstruktur der Probe mit ihrem elektronischen Kontrast.

Den Einfluss der Setzspannung auf die Abbildung einer Oberfläche mit dem STM verdeutlicht Abbildung 11. Die besetzten Zustände der Probe zwischen $E_{F}{ }^{S}$ und $E_{F}{ }^{T}$, aus denen Elektronen in unbesetzte Zustände der Spitze tunneln können, werden bei negativen Bias-Spannungen abgebildet. Im Gegensatz dazu werden bei positiven Bias-Spannungen die unbesetzten Zustände der Probe in dem Energieintervall zwischen $E_{F}{ }^{S}$ und $E_{F}{ }^{T}$ abgebildet.

(a)

$$
\mathrm{U}_{\text {Bias }}<0 \mathrm{~V}
$$

$$
\mathrm{U}_{\text {Bias }}>0 \mathrm{~V}
$$

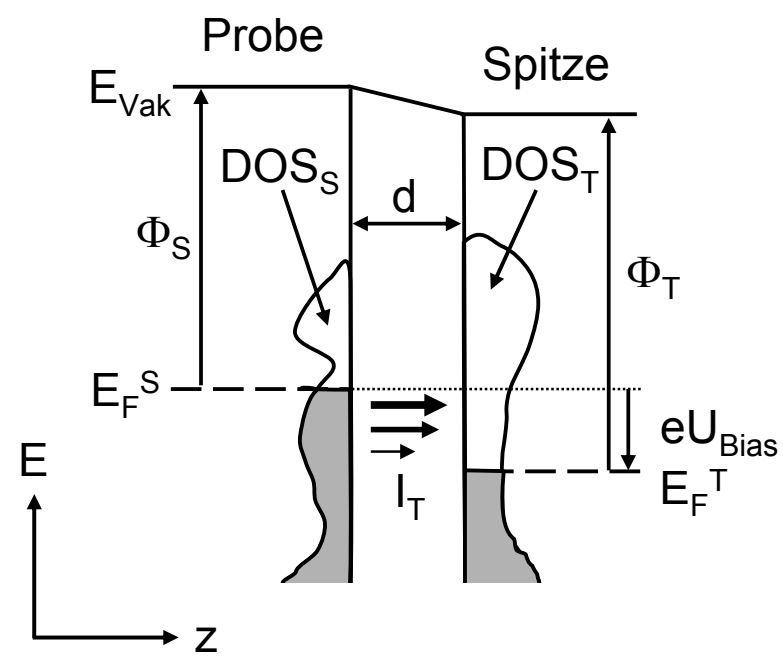

Abbildung 11: Schematische Darstellung des Tunnelkontakts bei (a) negativer und (b) positiver Bias-Spannung. Bei negativer Bias-Spannung kommt es zu Tunnelprozessen aus besetzten Zuständen der Probe in unbesetzte Zustände der Spitze. Bei positiver Bias-Spannung können Elektronen aus besetzten Zuständen der Spitze in unbesetzte Zustände der Probe Tunneln. 
Durch den Vergleich von Konstantstromtopografien, die bei unterschiedlichen Bias-Spannungen aufgenommen wurden, können daher Informationen über die Energieabhängigkeit der LDOS gewonnen werden. Nacheinander aufgenommene Topografien eignen sich für eine solche Analyse nur bedingt, da sie häufig aufgrund von geringfügiger thermischer Drift nicht exakt denselben Ausschnitt der Probenoberfläche zeigen. Um dieses Problem zu umgehen, ist es notwendig, einen Satz von Konstantstromtopografien simultan aufzunehmen. Dies gelingt näherungsweise im Multi-Bias Modus, bei dem jede Scan-Zeile zunächst bei verschiedenen Bias-Spannungen aufgenommen wird, bevor mit dem Scannen der nächsten Zeile begonnen wird. Der zeitliche Unterschied zwischen den Topografien entspricht in diesem Fall der Dauer, die zum Aufnehmen einer Scan-Zeile benötigt wird, so dass ein sehr guter räumlicher Zusammenhang besteht, der einen direkten Vergleich erlaubt.

Aufbauend auf dem Messmodus der Konstantstromtopografie haben sich eine Reihe spektroskopischer Messmethoden etabliert, auf die in den folgenden Abschnitten eingegangen wird.

\subsection{Raster-Tunnel-Spektroskopie}

Die Raster-Tunnel-Spektroskopie (STS) wurde entwickelt, um lokal energieaufgelöste Informationen über die LDOS der Probe zu gewinnen. Dazu wird zunächst der Abstand z zwischen Spitze und Probe an dem Messpunkt $(x, y)$ analog zu dem Konstantstrommodus bei einer Setzspannung und einem Setzstrom eingeregelt. Man spricht hierbei von dem Setzpunkt der Spektroskopie. Im nächsten Schritt wird bei ausgeschaltetem Regelkreis, d.h. bei einem festen Abstand zwischen Spitze und Probe, der Tunnelstrom als Funktion der Bias-Spannung in einem vorgegebenen Intervall aufgezeichnet. Da der Tunnelstrom bei der Untersuchung von Halbleitern in der Nähe der Bandkanten sehr gering wird, kann der Spitze-Probe Abstand durch einen z-Vorschub kontrolliert reduziert werden, während die I(U)Kennlinie aufgezeichnet wird. Die Dynamik der Messung kann auf diesem Weg deutlich erhöht werden. Eine typische Einstellung ist ein linearer Vorschub der Spitze mit kleiner werdender Probenspannung, so dass gemäß (2-1) der Tunnelstrom aufgrund der exponentiellen Abstandsabhängigkeit verstärkt wird.

Aus (2-7) geht hervor, dass es bei größeren Bias-Spannungen nicht mehr möglich ist, die LDOS direkt durch Differenzieren einer I(U)-Kennlinie zu bestimmen. Die differentielle Leitfähigkeit $d I / d U\left(U_{\text {Bias }}\right)$ entspricht in diesem Fall der Zustandsdichte der Probe, überlagert mit einer glatten, langsam mit $U_{\text {Bias }}$ variierenden Funktion [77]. Es ist daher üblich, die differentielle Leitfähigkeit zu normieren. Die gebräuchlichste Darstellungsform der spektroskopischen Informationen ist die von Feenstra vorgeschlagene normierte differentielle Leitfähigkeit [78]. Zur Normierung dient die totale Leitfähigkeit $I / U$, die proportional zum Transmissionskoeffizienten ist: 


$$
\frac{\left(\frac{d I}{d U}\right)}{\left(\frac{I}{U}\right)} \approx L D O S
$$

Obwohl es sich um eine zweckmäßige und weitgehend anerkannte Normierung handelt, ist auch die normierte differentielle Leitfähigkeit kein quantitatives Maß für die LDOS der Probe [77]. Dies liegt unter anderem daran, dass in der Regel weder die LDOS der Spitze noch die energieabhängige Transmission bekannt sind, die darüber hinaus durch eine lokal variierende Austrittsarbeit ortsabhängig sein kann.

\subsection{Spektroskopie der lokalen effektiven Barrierenhöhe}

Die lokale effektive Barrierenhöhe $\phi_{B}$ ist eine wichtige Größe bei der Auswertung von STM und STS Daten. Sie wird unter anderem benötigt, um Spektren gemäß den Gleichungen (2-1) und (2-2) auf einen einheitlichen Spitze-Probe Abstand zu normieren. Eine solche Normierung ist in der Regel notwendig, da der Tunnelabstand bei einer STS Messung über den Setzpunkt definiert wird und daher dem elektronischen Kontrast entsprechend lokal variiert. Zum anderen wird $\phi_{B}$ benötigt, um Spektren normieren zu können, die mit z-Vorschub aufgenommen wurden.

Um $\phi_{B}$ zu bestimmen, wird der Tunnelstrom gemessen, während der Spitze-Probe Abstand moduliert wird. $\phi_{B}$ kann anschießend aus diesen Daten berechnet werden:

$$
\phi_{B}=\frac{\hbar^{2}}{8 m}\left(\frac{d \ln I_{T}}{d z}\right)^{2}
$$

Die Messung der effektiven Barrierenhöhe kann darüber hinaus wichtige Informationen zur Bestimmung der Austrittsarbeit $\phi_{T}$ der Spitze liefern. In Kapitel 3.3.1 wird erläutert, dass die Kenntnis von $\phi_{T}$ eine Grundvoraussetzung darstellt, um sowohl STM als auch STS Untersuchungen von GaAs\{110\} Spaltflächen quantitativ analysieren zu können.

\subsection{Spitzenpräparation}

Die Spitze als Sonde des STMs spielt eine sehr wichtige Rolle im Zusammenspiel der verschiedenen, an der Messung beteiligten Komponenten. Schon die Tatsache, dass nur einige wenige Atome am äußersten Ende der Sonde letztendlich über die Qualität der Messung entscheiden, macht deutlich, wel- 
che Anforderungen an die Spitzenpräparation gestellt werden. Um möglichst stabile Spitzen herzustellen, die routinemäßig atomare Auflösung auf Halbleiterproben wie GaAs liefern, hat sich ein Prozess aus mehreren voneinander unabhängigen Schritten bewährt. ${ }^{7}$

Im ersten Schritt erhält der polykristalline Wolfram Draht, der einen Durchmesser von $250 \mu \mathrm{m}$ besitzt, in einem elektrochemischen Ätzvorgang seine makroskopische Form. Danach wird der Rohling in eine UHV Präparationskammer eingeschleust, in der er nachbearbeitet wird. Zunächst wird die den Rohling umgebende Oxidschicht entfernt, indem er über mehrere Minuten auf etwa $950^{\circ} \mathrm{C}$ aufgeheizt wird. Danach folgt ein etwa zwanzigminütiger frontaler Beschuss der Spitze mit Argon-Ionen, die Energien von typischerweise $4 \mathrm{keV}$ besitzen. Während dieses Prozessschritts werden sowohl eventuell noch verbliebene Reste der Oxidschicht entfernt, als auch bestimmte Kristallfacetten besonders herausgearbeitet. Im letzten Schritt vor der Verwendung im STM wird schließlich der Erfolg der vorangegangenen Prozesse überprüft, indem die Spitze über ihre Feldemission charakterisiert wird. Abbildung 12 zeigt eine Raster-Elektronen-Mikroskop Aufnahme einer typischen Spitze nach den beschriebenen UHV Präparationsschritten. Die für die STM Messung entscheidenden geometrischen Merkmale einer Spitze sind sowohl ihr Krümmungsradius als auch ihr Öffnungswinkel. Trotz des standardisierten Präparationsverfahrens besitzt jede Spitze individuell unterschiedliche Eigenschaften. In Ref. [79] konnte gezeigt werden, dass Spitzen nach den beschriebenen Präparationsschritten in der Regel Krümmungsradien von weniger als $10 \mathrm{~nm}$ und Öffnungswinkel von in der Regel weniger als 40 Grad besitzen.

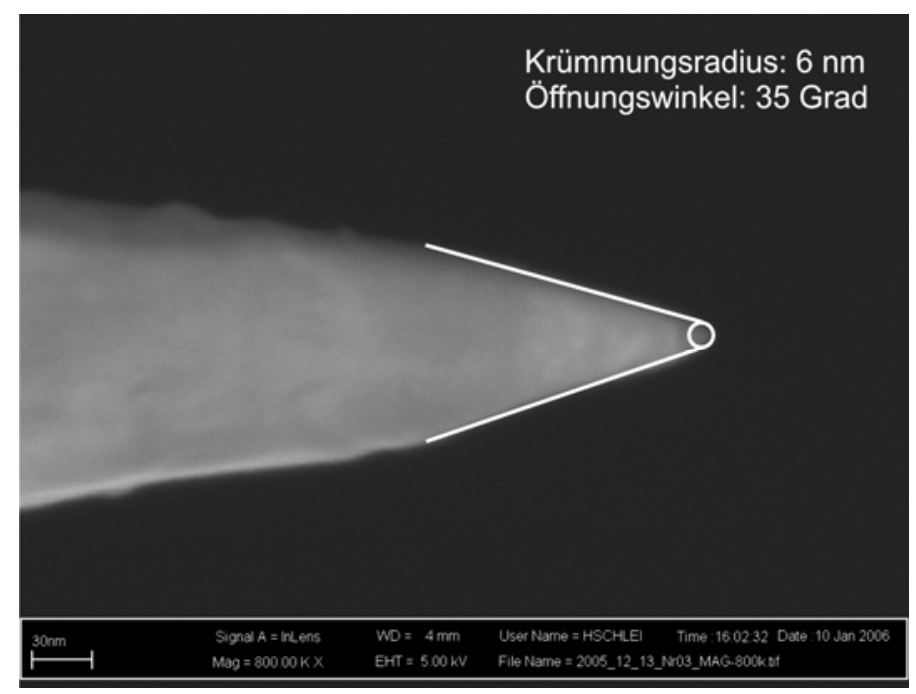

Abbildung 12: Aufnahme einer Wolfram-Spitze nach der UHV Präparation mit dem RasterElektronen-Mikroskop (aus [79]). Der Krümmungsradius der Spitze beträgt 6 nm, der Öffnungswinkel ist 35 Grad.

7 Eine detaillierte Beschreibung der Spitzenpräparation ist in verschiedenen Quellen zu finden [79, 86]. 


\section{STM auf Spaltflächen}

Die Raster-Tunnel-Mikroskopie ist dem Prinzip nach eine oberflächensensitive Methode. Informationen aus Bereichen unter der Oberfläche sind nur sehr bedingt zugänglich. Um das STM zur Untersuchung von Strukturen einsetzen zu können, die sich im Inneren der Probe befinden, bedient man sich einer speziellen Technik, bei der die Probe unter UHV Bedingungen gezielt gespalten wird. Auf diese Weise entstehen saubere, für das STM zugängliche Bruchflächen. Dieses Messverfahren wird als Querschnitts-Raster-Tunnel-Mikroskopie (engl. cross-sectional STM, XSTM) bezeichnet. Das Potential dieser Technik konnte bei der Aufklärung der atomaren und elektronischen Eigenschaften von einer Vielzahl unterschiedlicher Halbleiter Heterostrukturen bereits eindrucksvoll demonstriert werden [80-83].

Zu Beginn dieses Kapitels wird kurz auf die speziellen Anforderungen an den Aufbau eines XSTMs eingegangen. Im Anschluss werden die strukturellen und elektronischen Eigenschaften der im Zentrum dieser Arbeit stehenden GaAs\{110\} Spaltflächen vorgestellt. Am Beispiel der GaAs(110) Spaltfläche wird die Abbildung mit dem STM bei unterschiedlichen Bias-Spannungen unter Berücksichtigung der spitzeninduzierten Bandverbiegung ausführlich diskutiert, bevor auf die ersten STM Messungen von Metall-Halbleiter Kontakten in Querschnittsgeometrie eingegangen wird. Die vorliegende Arbeit baut an vielen Stellen auf Erkenntnissen aus diesen Experimenten auf, so dass neben den Ergebnissen auch die während der Messung und bei der Interpretation der Daten aufgetretenen Probleme diskutiert werden.

\subsection{Experimenteller Aufbau des XSTM}

Im Gegensatz zu herkömmlichen STM Systemen bietet das XSTM neben der Möglichkeit der vertikalen Positionierung der Spitze zur Grobannäherung an die Probe auch die Möglichkeit der lateralen Positionierung in einem relativ großen Bereich. Dies ist insbesondere bei der Untersuchung von Heterostrukturen unerlässlich, die sich in der Regel nur in einem bestimmten Bereich der zu untersuchenden Spaltfläche befinden. ${ }^{8}$

8 Die maximale vertikale Verschiebung beträgt $\pm 1,5 \mathrm{~mm}$, so dass lateral eine Fläche von etwa 16 $\mathrm{mm}^{2}$ erreicht werden kann. 


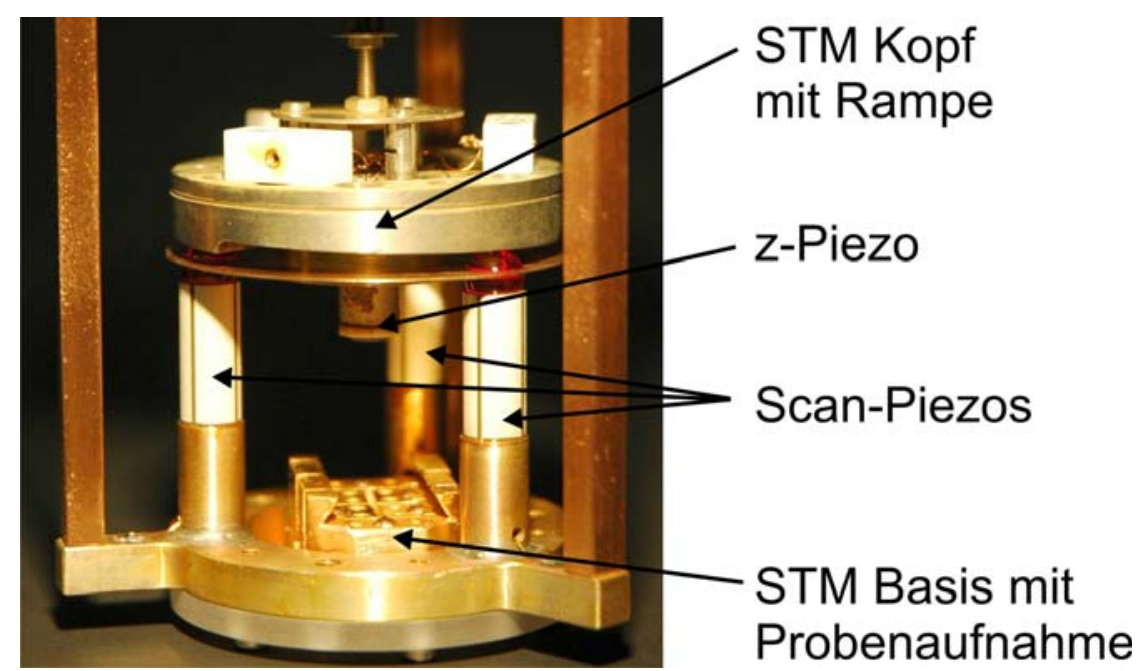

Abbildung 13: XSTM des Beetle-Typs, basierend auf dem Design von Besocke [84]. STM Kopf und Basis können über Slip-Stick-Bewegungen relativ zueinander verschoben werden. Die Probenaufnahme bietet die Möglichkeit, bis zu 6 elektrische Kontakte an die Probe heranzuführen.

Der Aufbau der XSTMs, mit denen alle in dieser Arbeit präsentierten STM Messungen durchgeführt worden sind, ist in Abbildung 13 dargestellt. Er basiert auf dem Design des von Besocke in Jülich entwickelten Beetle-Typs [84]. Ein wesentlicher Vorteil des Beetle-STMs ist die Möglichkeit, weite Bereiche der Probe erreichen zu können, ohne dazu auf mechanische Durchführungen zurückgreifen zu müssen. Dies wird dadurch erreicht, dass der STM Kopf und damit auch die Spitze relativ zur STM Basis, in der sich die Probe befindet, durch Slip-Stick-Bewegungen verschoben werden kann. Durch die an dem STM Kopf befindlichen Rampen kann durch eine Rotation des Kopfes relativ zur Basis eine grobe An- bzw. Abnäherung von Spitze und Probe erreicht werden. Durch die verschiedenen SlipStick-Bewegungen kann die Spitze so nahe an den zu untersuchenden Teil der Probenoberfläche heran manövriert werden, bis sie sich in dem Arbeitsbereich der Piezokeramiken befinden. Wie zu Anfang von Kapitel 2 beschrieben wird während der Messung in einem Rasterverfahren systematisch eine große Zahl von Messpunkten mit der Spitze sehr präzise über die Piezokeramiken angesteuert. Die laterale Bewegung übernehmen dabei die drei äußeren Scan-Piezos, während die vertikale mit dem z-Piezo des STM Kopfes erfolgt.

Um die Kontamination der Probenoberflächen möglichst gering zuhalten, sind die XSTMs in UHV Kammern integriert. Die in dieser Arbeit präsentierten STM Messungen wurden sowohl an einem bei RT arbeitenden System als auch an einem bei $6 \mathrm{~K}$ betriebenen Tieftemperatur STM durchgeführt. Beide Systeme wurden bereits im Detail in anderen Arbeiten beschrieben [73, 85, 86]. Die UHV Kammern, in denen routinemäßig Drücke von unter $3 \mathrm{e}^{-11}$ mbar erreicht werden, zeichnen sich durch ihre guten optischen Zugänge zum Bereich von Probe und Spitze aus. Sie erlauben die Verwendung eines 
externen Mikroskops zur Überwachung der Annäherung der Spitze an die Probe und zum besseren Auffinden der Heterostrukturen.

\subsection{Atomare und elektronische Eigenschaften der GaAs $\{110\}$ Spaltflächen}

Cross-sectional STM beruht auf der Möglichkeit, durch einen Spaltvorgang eine definierte Oberfläche zu erzeugen und so Strukturen zugänglich zu machen, die sich ursprünglich im Volumen der Probe befunden haben. Um mit dem STM effektiv Informationen gewinnen zu können, sollte die erzeugte Bruchfläche spezielle Anforderungen erfüllen. Zum einen sollte die Konfiguration der Atome in der neu entstanden Oberfläche die Volumenkonfiguration widerspiegeln und nicht rekonstruiert sein. Des Weiteren sollte der zu untersuchende Bereich der Spaltfläche für eine hochauflösende STM Untersuchung sauber sein und nur wenige, durch den Spaltvorgang hervorgerufene Defekte wie zum Beispiel Fehlstellen oder Stufen aufweisen. Durch einen Spaltvorgang im UHV lässt sich bei einer begrenzten Zahl von Probensystemen eine Oberfläche erzeugen, die diese Kriterien erfüllt. Es handelt sich hierbei um spröde Materialien, bei denen es bevorzugt zu einem transkristallinen Bruchverlauf entlang von natürlichen Spaltflächen kommt. Ein Beispiel ist GaAs mit seinen natürlichen \{110\} Spaltflächen, die sich ideal zur Untersuchung mit dem XSTM eignen [63, 73, 86, 87]. Auf der Basis eines üblicherweise verwendeten (001)-orientierten GaAs Wafers sind wie in Abbildung 14 gezeigt zwei orthogonal zueinander verlaufende Scharen von $\{110\}$ Bruchflächen zugänglich. Um ein Spaltvorgang auszulösen, wird der GaAs Wafer mechanisch belastet, wobei eine Bruchfläche bevorzugt in Bereichen entsteht, in denen die Struktur des Kristalls bereits geschwächt ist [73]. Dies kann dazu ausgenutzt werden, um gezielt eine der beiden orthogonalen Spaltflächen an einer bestimmten Position zu erzeugen.

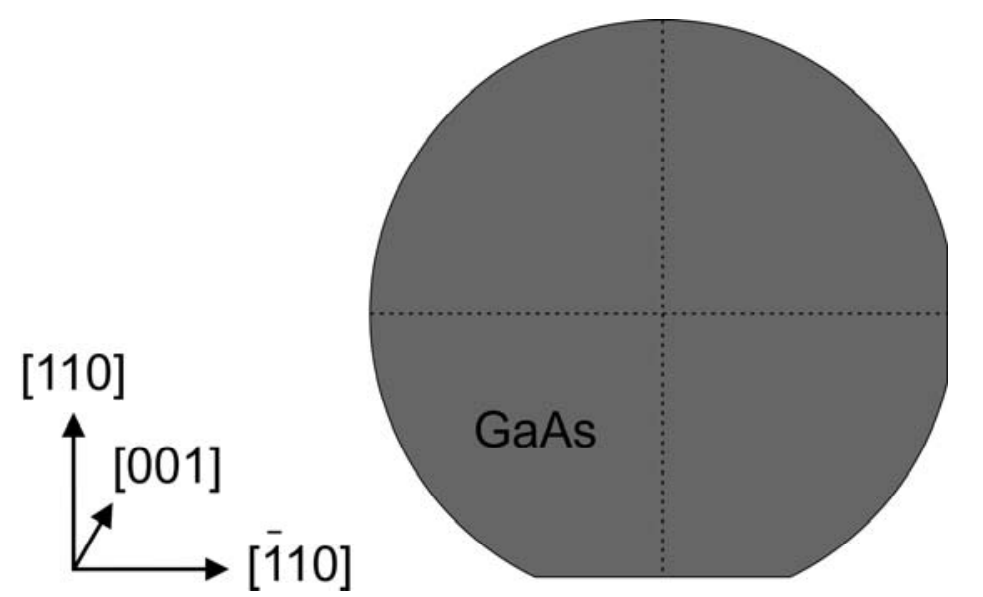

Abbildung 14: (001)-orientierter GaAs Wafer. Zwei orthogonal zueinander verlaufende \{110\} Bruchflächen sind durch die gepunkteten Linien angedeutet. 


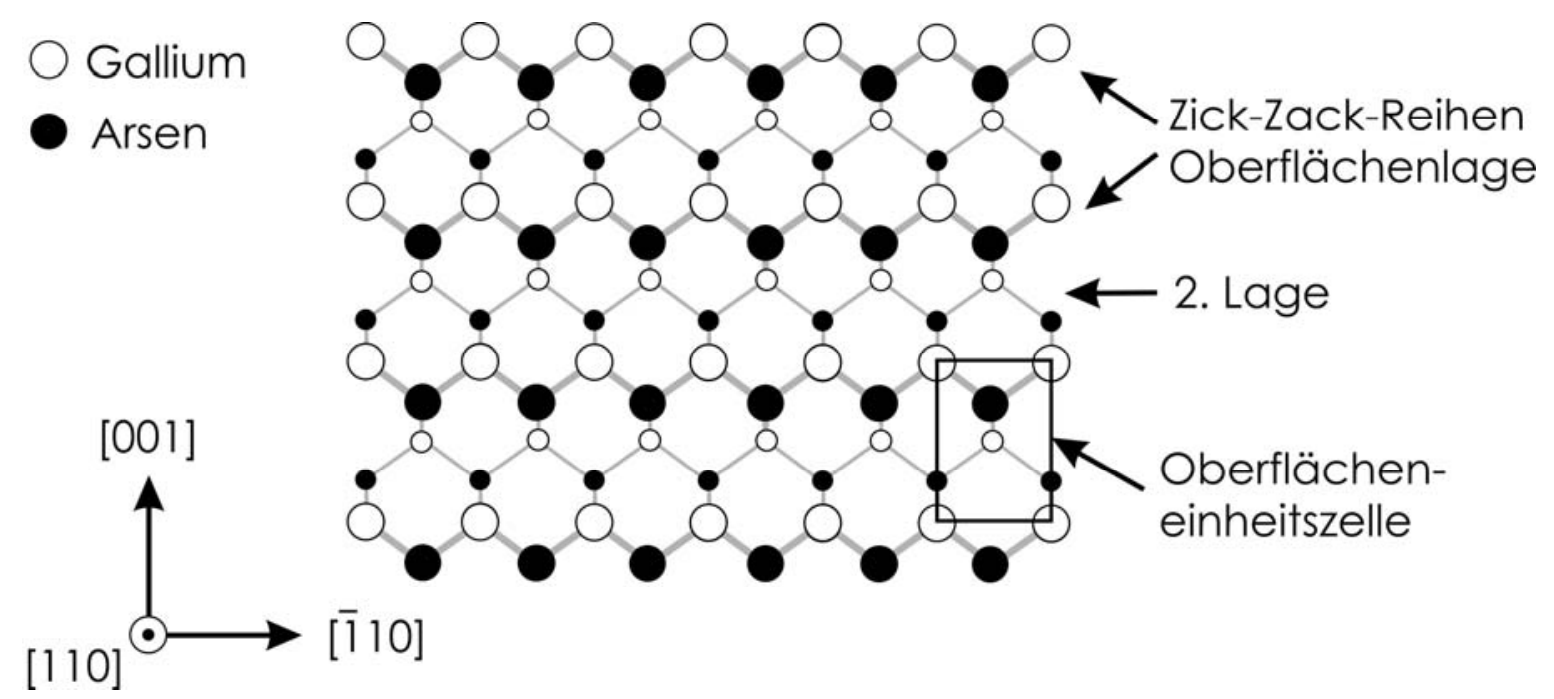

Abbildung 15: „Ball and Stick“ Modell der GaAs(110) Oberfläche (Oberflächenlage und zweite Lage). Die Oberflächeneinheitszelle ist eingezeichnet. Die alternierend aus Ga- und As-Atomen bestehenden Zick-Zack-Reihen in der Oberflächenlage sind gut zu erkennen. Die Oberflächeneinheitszelle von $4 \AA$ x 5,65 $\AA$ ist dargestellt.

Die Oberfläche der GaAs Spaltfläche setzt sich zu gleichen Teilen aus Ga- und As-Atomen zusammen. Diese sind alternierend in Zick-Zack-Reihen angeordnet, die senkrecht zur [001]-Richtung verlaufen. In Abbildung 15 ist dies am Beispiel der atomaren Struktur der GaAs(110) Oberfläche dargestellt.

Durch den Spaltvorgang werden 2 Bindungen pro Oberflächeneinheitszelle aufgebrochen, die als „dangling bonds“ (db) bezeichnet werden. Die ideale Struktur der Oberfläche, bei der sich die Atome an ihren Volumenpositionen befinden, ist energetisch ungünstig und daher instabil. Vor allem in der Oberflächenlage kommt es durch eine Veränderung der Bindungswinkel zu einer leichten Relaxation der Atome („,buckling“), die mit einer Ladungsumverteilung einhergeht [88].

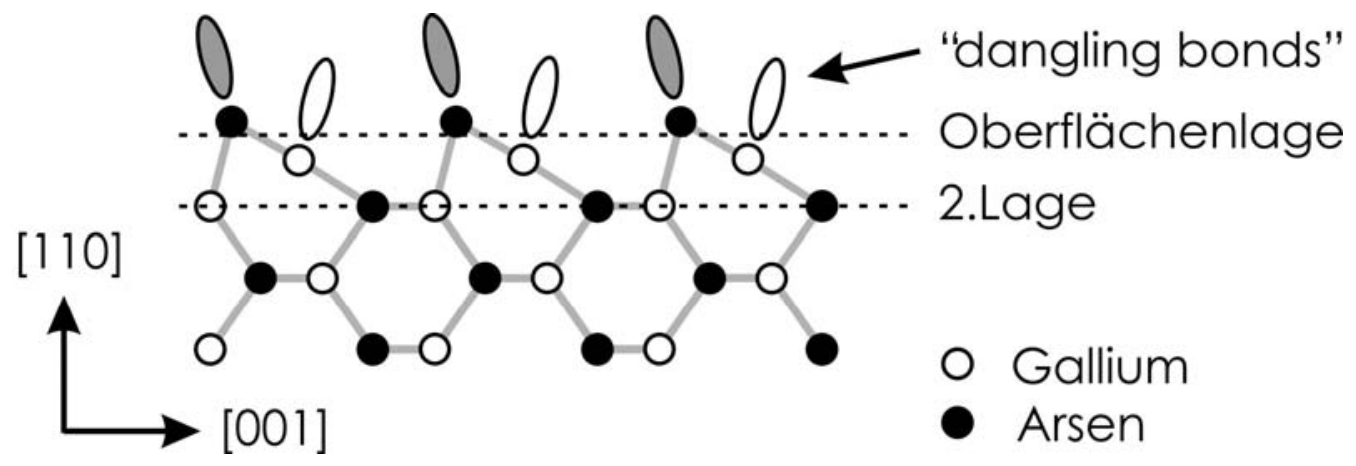

Abbildung 16: GaAs mit relaxierter Oberflächenlage. Die Position der As-Atome verschiebt sich aus der Oberfläche heraus während die Ga-Atome in die entgegengesetzte Richtung relaxieren. Die „dangling bonds“ über den As-Atomen sind besetzt, die der Ga-Atome unbesetzt. 
Wie in Abbildung 16 skizziert bewegen sich die an der Oberfläche befindlichen As-Atome leicht aus der Spaltebene heraus, während die Ga-Atome in die entgegengesetzte Richtung relaxieren. Chelikowsky hat auf der Basis von selbstkonsistenten Pseudopotentialen die durch die Relaxation veränderte LDOS an der GaAs(110) Oberfläche berechnet [89]. Die Zustände der relaxierten Oberfläche werden entsprechend ihrer räumlichen Lokalisierung im Bereich der Anionen ( $A_{4}$ und $A_{5}, A_{6}$ ) bzw. Kationen $\left(C_{3}\right.$ und $\left.C_{4}\right)$ bezeichnet. Abbildung 17 macht deutlich, dass sich die energetische Position der db-Zustände, die sich im Fall der unrelaxierten Oberfläche etwa in der Mitte der fundamentalen Bandlücke befinden würden, zu den Bandkanten hin verschiebt. Die besetzten db-Zustände der As-Atome befinden sich als Oberflächenresonanzen im Bereich des Valenzbands, während sich die freien dbZustände der Ga-Atome als Oberflächenresonanzen im Leitungsband befinden. Die Bandlücke an der Oberfläche ist dadurch nahezu identisch mit der fundamentalen Volumenbandlücke. Das Fehlen eines Oberflächenzustands ist das wohl markanteste und wichtigste Charakteristikum der GaAs\{110\} Spaltflächen, aus der sich sowohl wichtige Konsequenzen für die Abbildung mit dem STM (Kapitel 3.3) als auch für die Eignung als Substrat eines idealen Schottky-Kontakts ergeben (Kapitel 4.1).

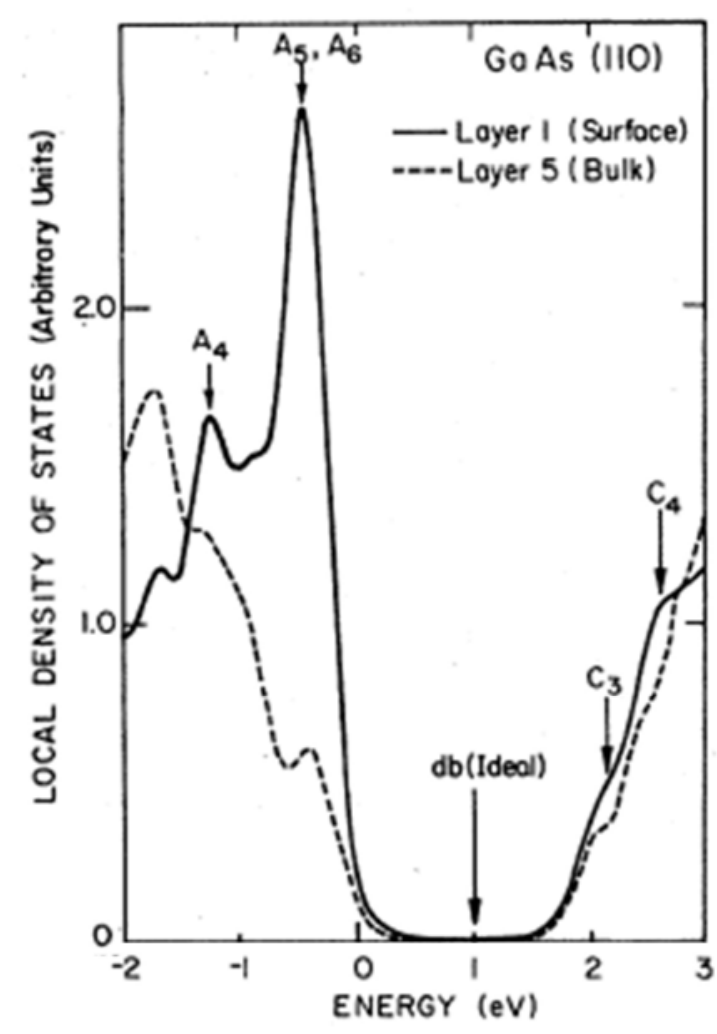

Abbildung 17: (aus [89]) Berechnete LDOS an der GaAs (110) Oberfläche und im Volumen im Energiebereich der fundamentalen Bandlücke. Die an den Anionen lokalisierten Oberflächenresonanzen $A_{4}, A_{5}$ und $A_{6}$ und die an den Kationen lokalisierten Zustände $C_{3}$ und $C_{4}$ der relaxierten Oberfläche sind eingezeichnet. Die Lage der db-Zustände ohne „buckling“ ist angegeben. 


\subsection{Die Abbildung der GaAs(110) Spaltfläche mit dem STM}

Entscheidend für die Abbildung mit dem STM ist gemäß (2-5) die integrale LDOS der Probe am Ort der Spitze. Da bei Messungen typischerweise Bias-Spannungen von bis zu einigen Volt angelegt werden, handelt es sich bei dem in Abbildung 17 dargestellten Energieintervall um den Bereich, der auch für STM Untersuchungen relevant ist. Der Vergleich der in Abbildung 17 dargestellten Volumen- und Oberflächen-LDOS macht bereits deutlich, wie stark die lokale Zustandsdichte der GaAs\{110\} Oberfläche im Bereich der beiden Bandkanten von den Oberflächenresonanzen dominiert wird. Die räumliche Variation der LDOS der Zustände $A_{4}, A_{5}, C_{3}$ und $C_{4}$ wurde für die (110) Oberfläche des III-V Halbleiters Indiumphosphit (InP) von Engels ab initio auf der Basis der Dichte-Funktional-Theorie (DFT) berechnet [90]. Qualitativ treffen die Ergebnisse für die (110) Oberflächen aller III-V Halbleiter zu. Abbildung 18 (a) zeigt die LDOS in einen Abstand von 3,6 A über der InP(110) Oberfläche in den Energieintervallen der verschiedenen Zustände.

(a) Ab initio Berechnung mit DFT
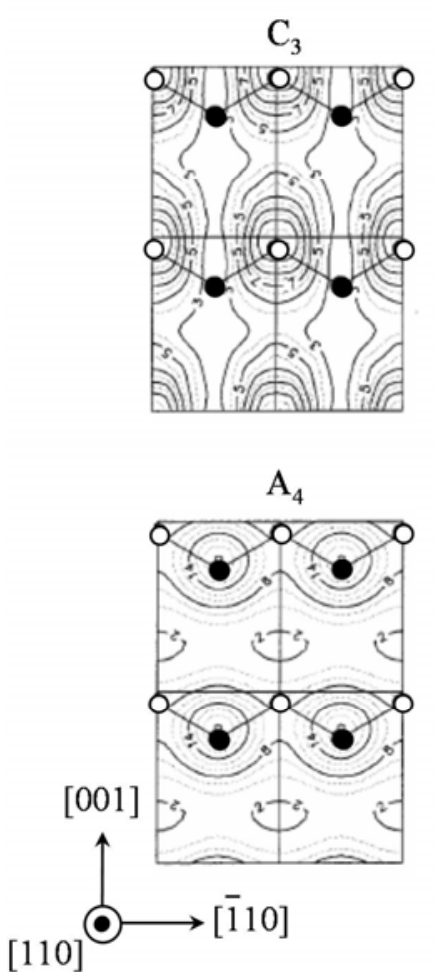
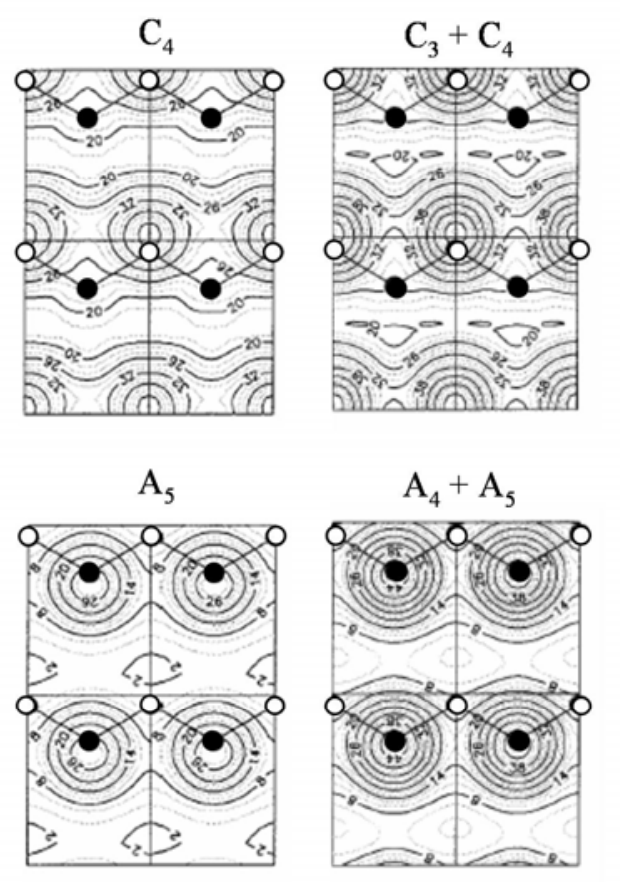

(b) Experiment
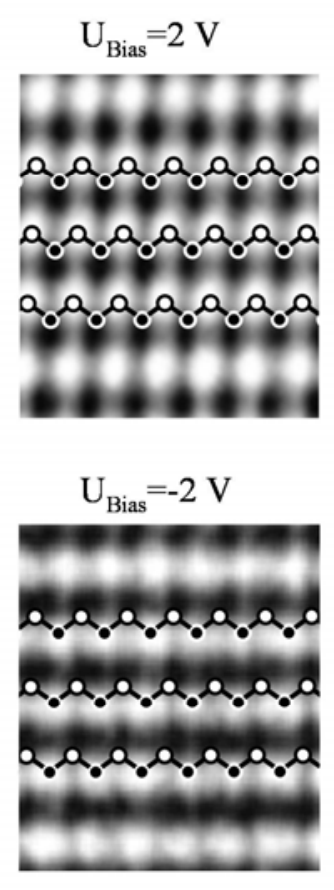

Abbildung 18: (a) zeigt die berechnete laterale Variation der integralen LDOS in einem Abstand von 3,6 Å über der InP(110) Oberfläche. (Die charakteristischen Energieintervalle der Zustände sind: $A_{5}$ : -0,95 eV bis $0 \mathrm{eV}$; $A_{4}$ : -1,77 eV bis -0,95 eV; $C_{3}: 0 \mathrm{eV}$ bis 2,27 eV; $C_{4}: 2,27 \mathrm{eV}$ bis 2,81 eV) Die Positionen der Anionen in der Oberflächenlage werden durch die schwarzen, die der Kationen durch die weißen Kreise symbolisiert (aus [90]). In (b) werden den berechneten lokalen Zustandsdichten entsprechende experimentelle Daten der GaAs(110) Oberfläche gegenüber gestellt, die im Rahmen dieser Arbeit aufgenommen wurden. 
Die räumliche Verteilung der Oberflächenzustände innerhalb der Oberflächeneinheitszelle variiert stark. Es wird deutlich, dass die besetzten $\mathrm{A}_{4}$ - und $\mathrm{A}_{5}$-Zustände an den Anionen der Oberflächenlage lokalisiert sind, während die freien Zustände $\mathrm{C}_{3}$ und $\mathrm{C}_{4}$ im Wesentlichen an den Kationen lokalisiert sind. Darüber hinaus ist in Abbildung 18 deutlich zu erkennen, dass sich die Korrugation der Zustände zum Teil stark unterscheidet. Charakteristisch ist hierbei, dass nur im Fall des $\mathrm{C}_{3}$-Zustands eine starke Korrugation in [1110]-Richtung auftritt. Bei den übrigen Zuständen dominiert die Korrugation senkrecht zu den Zick-Zack-Reihen in [001]-Richtung. Dem gegenübergestellt sind in Abbildung 18 (b) experimentelle Daten, die im Rahmen dieser Arbeit mit dem STM bei positiven und negativen BiasSpannungen gewonnen wurden. Die beiden atomar aufgelösten Topografien wurden im Multi-Bias Modus aufgenommen. Sie zeigen den identischen Ausschnitt der GaAs(110) Probenoberfläche und können daher direkt miteinander verglichen werden. Die spannungsabhängigen Veränderungen in der Morphologie der Topografien sind qualitativ in guter Übereinstimmung mit den Berechnungen von Engels - bei positiven Probenspannungen werden die freien, an den Kationen lokalisierten Zustände abgebildet, bei negativen Spannungen dominieren die besetzten A-Zustände die Abbildung. Auch der charakteristische Verlauf der Korrugation ist in guter Übereinstimung mit den theoretischen Kalkulationen. Es wird deutlich, dass

- die Korrugation in STM Messungen die zugrunde liegende atomare Struktur der Oberfläche widerspiegelt

- durch die Wahl der zur Abbildung mit dem STM gewählten Bias-Spannung selektiv das As-Untergitter bzw. das Ga-Untergitter adressiert werden kann.

- durch den Verlauf der atomaren Korrugation auf den dominanten Tunnelkanal geschlossen werden kann.

Insbesondere die Möglichkeit, das As- und Ga-Untergitter selektiv abzubilden, kann die Charakterisierung von Defekten in der atomaren Struktur einer Oberfläche entscheidend erleichtern (siehe Kapitel $6)$.

\subsubsection{Einfluss der spitzeninduzierten Bandverbiegung}

Die experimentell beobachtete starke Abhängigkeit der Morphologie der GaAs\{110\} Oberfläche von der gewählten Probenspannung kann qualitativ sehr gut durch die räumliche Variation der integralen LDOS innerhalb der Oberflächeneinheitszelle erklärt werden. Das Spannungsintervall, in dem ein Zustand experimentell beobachtet werden kann, stimmt allerdings in der Regel nicht mit dem überein, dass man anhand der energetischen Lage des Zustands erwarten würde [91].

Üblicherweise wird davon ausgegangen, dass die Anwesenheit der STM Spitze über einer Probenoberfläche als eine geringfügige Störung des zu untersuchenden Systems betrachtet werden kann. Die ein- 
gangs beschriebene Diskrepanz ist ein Ausdruck davon, dass diese Annahme im Fall von STM Untersuchungen der GaAs\{110\} Spaltflächen nicht gerechtfertigt ist. Die starke Invasivität der STM Messung kann in Analogie zu dem Gedankenexperiment erklärt werden, das in Kapitel 1.2 im Rahmen der Schottky-Mott-Theorie beschrieben wurde. Abbildung 19 zeigt schematisch die Situation einer metallischen Spitze mit der Austrittsarbeit $\phi_{T}$ im Tunnelabstand vor einer n-GaAs\{110\} Spaltfläche mit der Elektronenaffinität $\chi_{s}$ bei unterschiedlichen Bias-Spannungen.

(a) $\quad U_{\text {Bias }}=0 \mathrm{~V}$

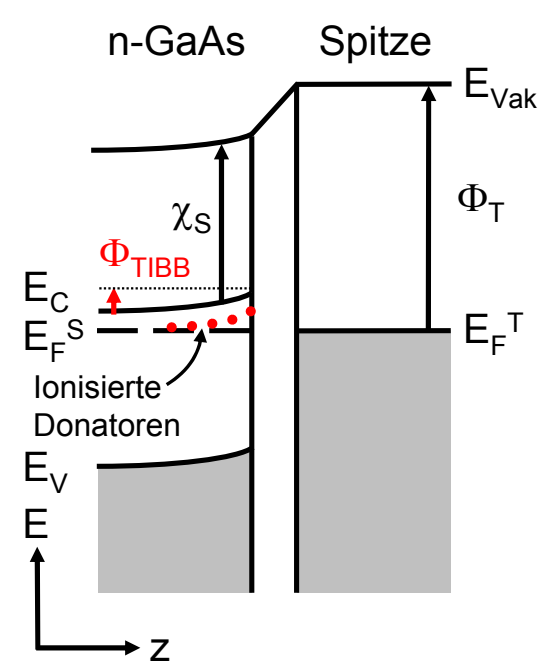

(b)

$\mathrm{U}_{\text {Bias }}>0 \mathrm{~V}$

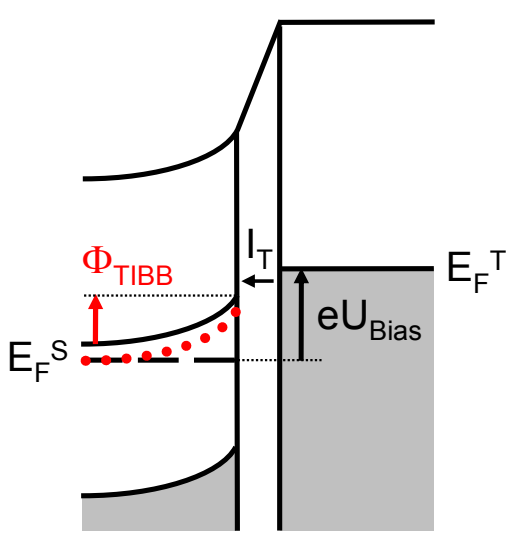

(c) $\quad U_{\text {Bias }}<0 \mathrm{~V}$

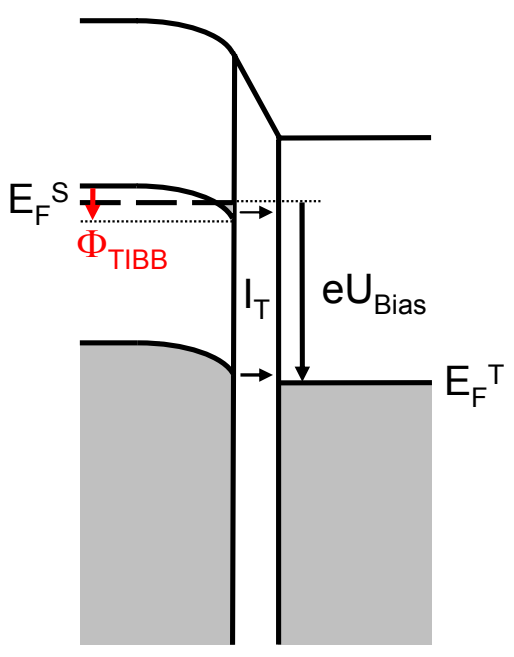

Abbildung 19: Schematische 1D-Bandmodelle des Tunnelexperiments bei verschiedenen BiasSpannungen. Die metallische Spitze mit ihrer Austrittsarbeit $\phi_{T}$ ist durch die Vakuumbarriere von der $n$-GaAs Spaltfläche mit ihrer Elektronenaffinität $\chi_{s}$ getrennt. Die spitzeninduzierte Bandverbiegung $\phi_{T I B B}$, die unter anderem von der gewählten Bias-Spannung abhängig ist, ist jeweils qualitativ eingezeichnet.

Im Allgemeinen sind die Austrittsarbeiten von Spitze und Probe unterschiedlich. In dem in Abbildung 19 dargestellten Beispiel ist $\phi_{T}>\phi_{S} \cdot{ }^{9}$ In Abbildung 19 (a) befinden sich Spitze und n-GaAs Spaltfläche ohne angelegte Bias-Spannung im Tunnelkontakt. Es kommt zum Austausch von Ladungsträgern, bis sich die mit $E_{F}{ }^{T}$ und $E_{F}{ }^{S}$ bezeichneten Fermi-Niveaus der beiden Subsysteme bei derselben Energie befinden. Die GaAs\{110\} Spaltflächen besitzen keinen Oberflächenzustand, der das elektri-

\footnotetext{
${ }^{9}$ Die Diskussion des umgekehrten Falls kann in Analogie geführt werden. Es kommt allerdings ohne Bias-Spannung zunächst zu einer negativen Bandverbiegung, so dass die Flachbandbedingung bei einer anderen Probenspannung erreicht wird.
} 
sche Feld der negativen Oberflächenladungsdichte in der STM Spitze bereits an der Oberfläche des Halbleiters effektiv abschirmen könnte [89]. Es kommt zur Ausbildung einer RLZ, in der die ionisierten, positiv geladenen Donatoren das elektrische Feld abschirmen. Die elektrostatische Energie $\phi_{\text {TIBB }}$, um die die mit $E_{C}$ und $E_{V}$ bezeichneten Leitungs- und Valenzbandkanten an der Oberfläche relativ zu ihrer Volumenposition verschoben werden, wird als spitzeninduzierte Bandverbiegung (engl. tip induced band bending, TIBB) bezeichnet.

Abbildung 19 (b) macht deutlich, dass das TIBB nicht nur von dem Abstand zwischen Probe und Spitze abhängig ist (siehe Gedankenexperiment in Kapitel 1.2), sondern auch von der angelegten BiasSpannung. Dies hat zur Folge, dass immer nur ein Teil des Potentials, das während der STM Messung zwischen Probe und Spitze angelegt wird, über der Vakuumbarriere abfällt. Dementsprechend ist es nicht möglich, einfach über die angelegte Bias Spannung das Energieintervall zu bestimmen, in dem Zustände an dem Tunnelprozess beteiligt sind.

In Abbildung 19 (c) ist die Situation für eine negative Probenspannung skizziert. Die STM Spitze besitzt eine positive Oberflächenladungsdichte, so dass die Bänder im Halbleiter zu niedrigeren Energien hin verschoben werden. An der GaAs Oberfläche befindet sich das Leitungsbandminimum $E_{C}$ unter der Fermi-Energie $E_{F}{ }^{S}$ und es kommt zu einer Akkumulation von Majoritätsladungsträgern, durch die das elektrische Feld abgeschirmt wird. Insbesondere bei hohen negativen Bias Spannungen kann es dazu kommen, dass neben den besetzten Zuständen aus dem Leitungsband auch besetzte Zustände aus dem Valenzband zu dem Tunnelstrom beitragen. Das Gewicht dieser beiden Beiträge verändert sich in Abhängigkeit von der gewählten Probenspannung, so dass bei geringen negativen Spannungen in der Regel das Ga-Untergitter und bei großen negativen Spannungen das As-Untergitter abgebildet wird.

Anhand des in Abbildung 19 gezeigten Beispiels wird deutlich, wie stark STM Messungen auf GaAs $\{110\}$ Spaltflächen durch das TIBB beeinflusst werden können. Eine genaue Kenntnis des TIBB als Funktion der Bias-Spannung ist daher eine Grundvoraussetzung, um STM Daten insbesondere im Hinblick auf die elektronischen Eigenschaften der untersuchten Probe analysieren zu können. Üblicherweise wird davon ausgegangen, dass das TIBB in guter Näherung auf der Basis eines einfachen 1D-Modells berechnet werden kann, bei dem die Oberflächen von Spitze und Probe als planparallel und unendlich ausgedehnt angenommen werden [72, 91-93]. Für die Austrittsarbeiten von Spitze und Probe werden Literaturwerte verwendet und es wird ein mittlerer Tunnelabstand $d$ angenommen. Die Ladungsdichte $\rho$ in der GaAs Probe wird in Abhängigkeit von der Messtemperatur im Rahmen der Effektivmassen-Näherung beschrieben. Der Verlauf des elektrostatischen Potentials $V(z)$ sowie die Ladungsdichte $\rho(z)$ im Halbleiter werden selbstkonsistent berechnet, so dass die Poisson-Gleichung (1-3) erfüllt wird. Die Aussagekraft der auf diese Art berechneten Werte des TIBB ist allerdings aufgrund der groben Näherungen und gemachten Annahmen stark eingeschränkt: 
- Der Tunnelabstand $d$ ist bei einer STM Messung prinzipiell nicht bekannt, so dass der Anteil des Potentialabfalls in der Vakuum-Barriere nur ungenau bestimmt werden kann.

- Die tatsächliche Austrittsarbeit der Spitze entspricht im Allgemeinen nicht dem Literaturwert des Spitzenmaterials [80]. Der Literaturwert bezieht sich auf eine saubere Oberfläche einer bestimmten Orientierung, was in der Regel bei den in dieser Arbeit verwendeten STM Spitzen nicht gegeben ist. Sie werden aus einem polykristallinen W-Draht geätzt, so dass die Orientierung der Kristallfacette am Apex der Spitze nicht bekannt ist. Zum anderen ist die Oberfläche der Spitze stark facettiert, was zu einer Absenkung der Austrittsarbeit führt [94]. Darüber hinaus können sich während der STM Messung Adsorbate an dem Spitzenapex anlagern und auch dadurch die Austrittsarbeit der Spitze stark verändern. Da der Wert der Austrittsarbeit bestimmt, bei welcher BiasSpannung der Übergang zwischen dem Akkumulations- und dem Verarmungsregime stattfindet, hat eine Abweichung vom Literaturwert hat zur Folge, dass das TIBB in einem gewissen Spannungsintervall nicht einmal qualitativ richtig berechnet wird. Dies kann zu erheblichen Fehlern bei der Interpretation der STM Daten führen.

Eine Alternative zur Verwendung des Literaturwertes besteht darin, die Größenordnung der Spitzenaustrittsarbeit aus STM Messungen der lokalen Barrierenhöhe (siehe Kapitel 2.5) zu extrahieren [95]. Eine exakte Bestimmung der Austrittsarbeit kann aber auch auf diesem Weg nicht erreicht werden. Zum einen wird die Spektroskopie der lokalen Barrierenhöhe selbst durch das TIBB modifiziert, dessen Wert nicht bekannt ist. Zum anderen wurde gezeigt, dass die gemessene effektive Barrierenhöhe insbesondere bei hohen Bias-Spannungen, wie sie gewöhnlich bei STM Messungen auf Halbleiteroberflächen verwendet werden, durch Bildladungseffekte um bis zu 30 \% von der Höhe der Rechteckbarriere abweichen kann [96].

- Die Spitzengeometrie hat einen starken Einfluss auf das TIBB, der in dem 1D-Modell nicht berücksichtigt wird. Die Berechnung des TIBB im Rahmen des 1D-Modells kann daher nur bei Tunnelspitzen mit extrem großen Krümmungsradien realistische Werte liefern. Um das TIBB auch für Spitzen mit geringen Krümmungsradien quantitativer berechnen zu können, ist es notwendig, die Potentiallandschaft für eine metallische Spitze im Abstand von wenigen Ångström vor einer halbleitenden Probe dreidimensional zu berechnen. Feenstra hat einen Lösungsansatz entwickelt, der dies unter der Voraussetzung radialer Symmetrie des Systems aus Spitze und Probe erlaubt [97]. Bei einer Spitze mit einem Krümmungsradius von $10 \mathrm{~nm}$ und einem Öffnungswinkel von 40 Grad, wie sie typischerweise im Rahmen dieser Arbeit verwendet wird, ist das TIBB um etwa einen Faktor 4 geringer, als es auf der Basis des 1D-Modells berechnet wird [80]. Diese Diskrepanz macht deutlich, dass eine Berechnung des TIBB generell in 3D durchgeführt werden sollte.

Trotz der konzeptionellen Fortschritte bei der 3D-Berechnung und der näherungsweisen Bestimmung der Spitzenaustrittsarbeit über die effektive Barrierenhöhe bleibt das TIBB in der Regel eine nur unzu- 
reichend bekannte Größe, da weder der Tunnelabstand noch die Geometrie der Spitze oder ihre Austrittsarbeit während der Messung bekannt sind.

Im Verlauf dieser Arbeit wird gezeigt, dass es allerdings in Spezialfällen möglich ist, durch den Vergleich von Messdaten mit 3D-Simulationen des TIBB die unbekannten Parameter zu rekonstruieren, so dass das TIBB auch quantitativ in die Analyse der Messdaten mit einbezogen werden kann (siehe Kapitel 7).

\subsubsection{Simulation des Tunnelstroms bei STM Messungen auf GaAs $\{110\}$ Spaltflächen}

Theoretische Berechnungen des Tunnelstroms können häufig einen essentiellen Beitrag zum grundlegenden Verständnis von STM Messungen leisten. Das von Koenraad und Mitarbeitern entwickelte Programm IVCHAR wurde bereits in verschiedenen Arbeiten erfolgreich eingesetzt, um experimentelle Tunnelspektren zu simulieren, die bei STS Messungen von GaAs\{110\} Spaltflächen aufgenommen wurden [91, 93, 98-100]. Da IVCHAR auch im Rahmen dieser Arbeit verwendet wurde, wird im Folgenden kurz auf die Grundzüge des Programms eingegangen. Für ausführlichere Beschreibungen stehen verschiedene Quellen zur Verfügung [93, 100].

Der verwendete Algorithmus basiert auf einem Ansatz von Feenstra und Stroscio, bei dem das System aus STM Spitze, Vakuumbarriere und Probe in einer 1D-Näherung behandelt wird [72]. Im ersten Schritt der Simulation wird der Verlauf des elektrostatischen Potentials $V(z)$ wie in Kapitel 3.3.1 beschriebenen in Abhängigkeit von der Bias-Spannung selbstkonsistent durch Lösen der PoissonGleichung (1-3) berechnet. Dabei können unter anderem die folgenden Parameter frei variiert werden:

- Tunnelabstand $d$

- Spitzenaustrittsarbeit $\phi_{T}$

- Fläche der Spitze $A_{T}$, durch die der Tunnelstrom fließt

- Volumenkonzentration von Dotieratomen, Ionisierungsenergie

- Temperatur, bei der die Messung durchgeführt wurde

Nachdem der Potentialverlauf unter Berücksichtigung des Einflusses der STM Spitze (TIBB) bestimmt wurde, wird im zweiten Schritt die Tunnelwahrscheinlichkeit als Funktion der Energie in dem durch die Bias-Spannung vorgegebenen Intervall im Rahmen der WKB Näherung berechnet. Eine gegebenenfalls auftretende Akkumulation von Ladungsträgern an der Probenoberfläche wird in Form eines 2D-Elektronen- bzw. Lochgases beschrieben. Über den Parameter $A_{T}$ wird schließlich der Tunnelstrom $I_{T}$ als Funktion der Bias-Spannung bestimmt. 
(a)

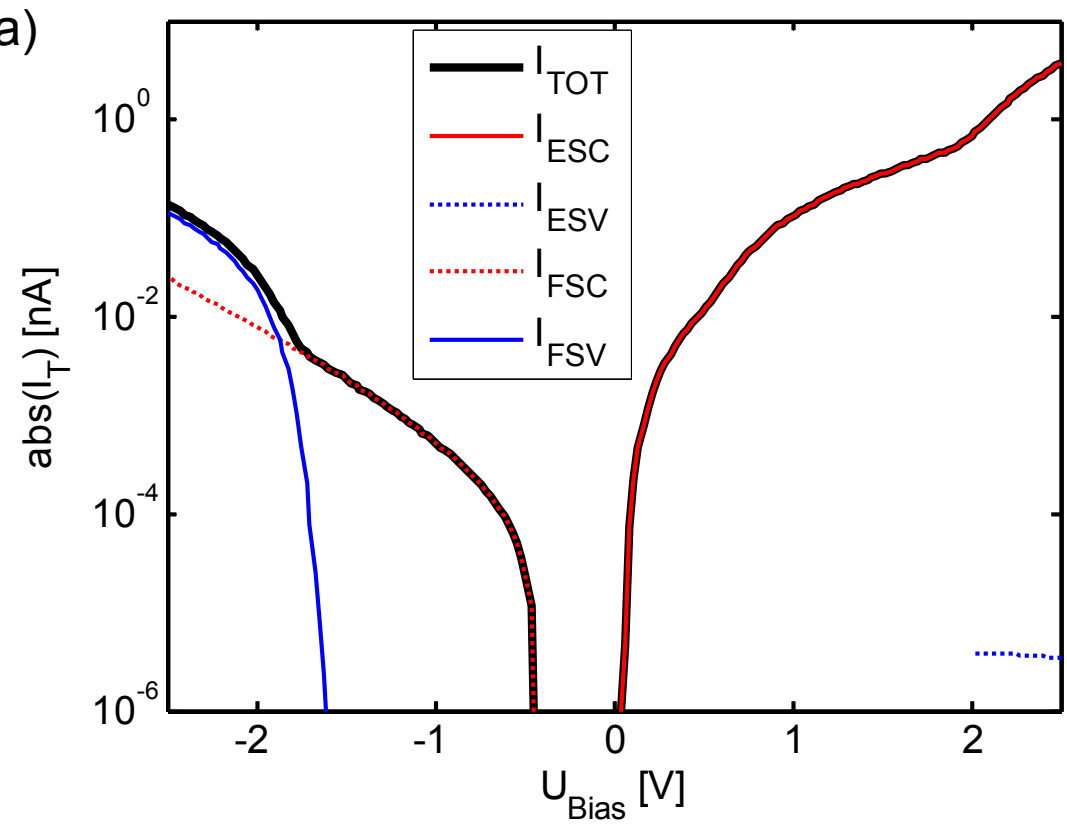

(b)

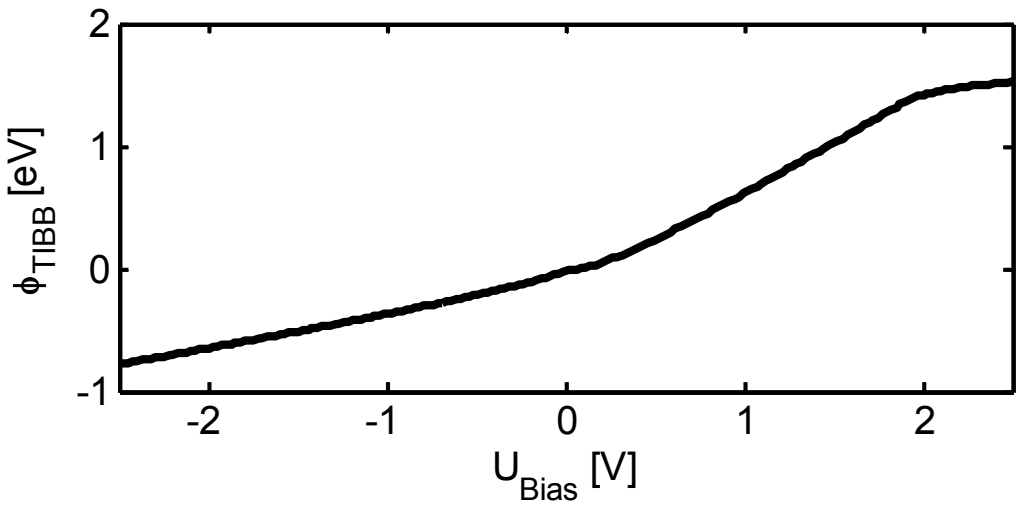

Abbildung 20: (a) Mit IVCHAR berechnete I(U)-Kennlinie in logarithmischer Darstellung. In Abhängigkeit von der Bias-Spannung tragen zu dem Gesamttunnelstrom $I_{\text {тот }}$ leere Leitungsbandzustände $\left(I_{E S C}\right)$, leere Valenzbandzustände $\left(I_{E S V}\right)$, gefüllte Leitungsbandzustände $\left(I_{F S C}\right)$ und gefüllte Valenzbandzustände $\left(I_{F S V}\right)$ bei. (b) $\phi_{\text {TIBB }}$ als Funktion der Bias-Spannung.

Eine solche mit IVCHAR berechnete I(U)-Kennlinie ist in Abbildung 20 (a) dargestellt. Sie wurde für eine bei RT durchgeführte STS Untersuchung einer n-GaAs\{110\} Spaltfläche simuliert $\left(8 \cdot 10^{17} 1 / \mathrm{cm}^{3}\right.$ Si dotiert, $\left.d=8 \AA, \phi_{T}=4 \mathrm{eV}, A_{T}=10 \AA^{2}\right)$. Die Parameter der Simulation wurden in Anlehnung an die Bedingungen gewählt, unter denen die in Kapitel 6 beschriebenen STM Datensätze aufgenommen wurden. Da $\phi_{T}$ in etwa der Elektronenaffinität $\chi_{S}$ von 4,07 eV entspricht, wird schon ab einer geringen positiven Probenspannung ein Tunnelstrom in leere Leitungsbandzustände $\left(I_{E S C}\right)$ berechnet. $I_{T}$ steigt mit zunehmender Bias-Spannung nur vergleichsweise langsam an, da der Anteil des zwischen Probe und Spitze angelegten Potentials, der über der Vakuumbarriere abfällt, durch das 
in Abbildung 20 (b) gezeigte TIBB nur langsam zunimmt. Bei einer Bias-Spannung von etwa $2 \mathrm{~V}$ ist $\phi_{\text {TIBB }}$ bereits so stark angestiegen, dass sich die Valenzbandkante über der Fermi-Energie der Probe befindet. Es kommt zur Akkumulation von Löchern an der Probenoberfläche, durch die das elektrische Feld zwischen Spitze und Probe effektiver abgeschirmt wird. Das TIBB vergrößert sich bei weiter steigenden Bias-Spannungen kaum noch, so dass in der I(U)-Kennlinie in Abbildung 20 (a) ein starkes Ansteigen des Gesamttunnelstroms $I_{\text {TOT }}$ zu erkennen ist, obwohl $I_{E S V}$ selbst kaum zu $I_{\text {ТОT }}$ beiträgt. Darüber hinaus geht aus der Simulation hervor, dass das Spannungsintervall, in dem keine Tunnelprozesse stattfinden können, deutlich kleiner als die GaAs Bandlücke von 1,43 eV bei RT ist. Durch das TIBB existiert bereits ab einer Bias-Spannung von etwa -0,5 V eine Akkumulationszone, aus der Elektronen in unbesetzte Zustände der Probe tunneln können $\left(I_{F S C}\right)$. Der Betrag von $\phi_{\text {TIBB }}$ nimmt bei steigenden negativen Bias-Spannungen weniger stark zu als bei positiven Bias-Spannungen, da das elektrische Feld zwischen Spitze und Probe durch die Elektronen in der Akkumulationszone effektiver abgeschirmt wird als durch die ionisierten Donatoren bei positiven Bias-Spannungen. Erst bei größeren negativen Bias-Spannungen können auch besetzte Zustände aus dem Valenzband $\left(I_{F S V}\right)$ zu $I_{\text {TOT }}$ beitragen.

Die Ergebnisse der IVCHAR Simulation besitzen in erster Linie eine qualitative Aussagekraft, weil der Einfluss der STM Spitze auf die Eigenschaften der zu untersuchenden Probe im Rahmen der 1D-Näherung nur unzureichend modelliert werden kann. Da in dieser Arbeit STM Spitzen mit Krümmungsradien von unter $10 \mathrm{~nm}$ verwendet wurden, wird die Ausprägung des TIBB bei der IVCHAR Simulation im Vergleich zum Experiment stark überbewertet (siehe Kapitel 3.3.1). Dadurch ergeben sich bei der Berechnung des Tunnelstroms im Fall der oben genannten Simulationsparameter die folgenden Tendenzen, die bei der Übertragbarkeit der Simulation auf die experimentellen I(U)Kennlinien beachtet werden müssen:

- Der Anstieg des Tunnelstroms bei positiven Bias-Spannungen wird durch die 1D-Simulation unterschätzt

- Im Experiment wird es erst ab einer erheblich höheren positiven Bias-Spannung zu einer Lochakkumulation kommen, als es durch die Simulation berechnet wird.

- Das Spannungsintervall, in dem experimentell kein Tunnelstrom beobachtet werden kann, ist größer als durch die Simulation berechnet, da es tatsächlich erst ab größeren negativen BiasSpannungen zur Akkumulation von Elektronen an der Probenoberfläche kommt, durch die der Einsatzpunkt des Tunnelstroms bestimmt wird.

- Durch ein geringeres TIBB trägt $I_{F S V}$ schon ab geringeren negativen Bias-Spannungen zu $I_{\text {TOT }}$ bei. 
Durch eine an das Experiment angepasste 3D-Berechnung des TIBB, die z.B. im Fall einer homogenen Probe mit der in Kapitel 3.3.1 beschriebenen Methode von Feenstra [97] erreicht werden kann, können verschiedene Aspekte der 1D-Simulation auf das Experiment übertragen werden. Hierzu zählten unter anderem die Bias-Spannungen, bei denen sich die Probe im Regime der Akkumulation befindet. Im Fall einer Spitzenaustrittsarbeit $\phi_{T}$ von etwa $4 \mathrm{eV}$ und einem Spitzenradius von unter 10 nm können die Tunnelbedingungen bei STM Messungen der GaAs\{110\} Spaltflächen wie folgt identifiziert werden:

- Bei positiven Bias-Spannungen von bis zu 3 V tragen ausschließlich Tunnelprozesse in leere Leitungsbandzustände $\left(I_{E S C}\right)$ zu dem Tunnelstrom bei.

- Bei negativen Bias-Spannungen wird der Einsatzpunkt des Tunnelstroms durch Tunnelprozesse aus der Akkumulationszone $\left(I_{F S C}\right)$ bestimmt.

- Die gefüllten Valenzbandzustände $\left(I_{F S V}\right)$ tragen erst bei größeren negativen Bias-Spannungen zu dem Gesamttunnelstrom bei.

\subsection{XSTM von Schottky-Kontakten}

Die Möglichkeiten der Querschnitts-Raster-Tunnel-Mikroskopie, insbesondere bei der Aufklärung der atomaren und elektronischen Eigenschaften von Halbleiter Heterostrukturen, wurden bereits an einer Vielzahl unterschiedlicher Systeme demonstriert [80-83]. Die prinzipielle Durchführbarkeit von STM Messungen an Metall-Halbleiter Kontakten in Querschnittsgeometrie konnte erstmals in der eigenen Arbeitsgruppe im Rahmen der Dissertation T.C.G. Reusch / Diplomarbeit L. Winking an einem einfachen Modellsystem gezeigt werden [93, 99, 101]. Die zu diesem Zweck entwickelte Messanordnung ist in Abbildung 21 dargestellt. 


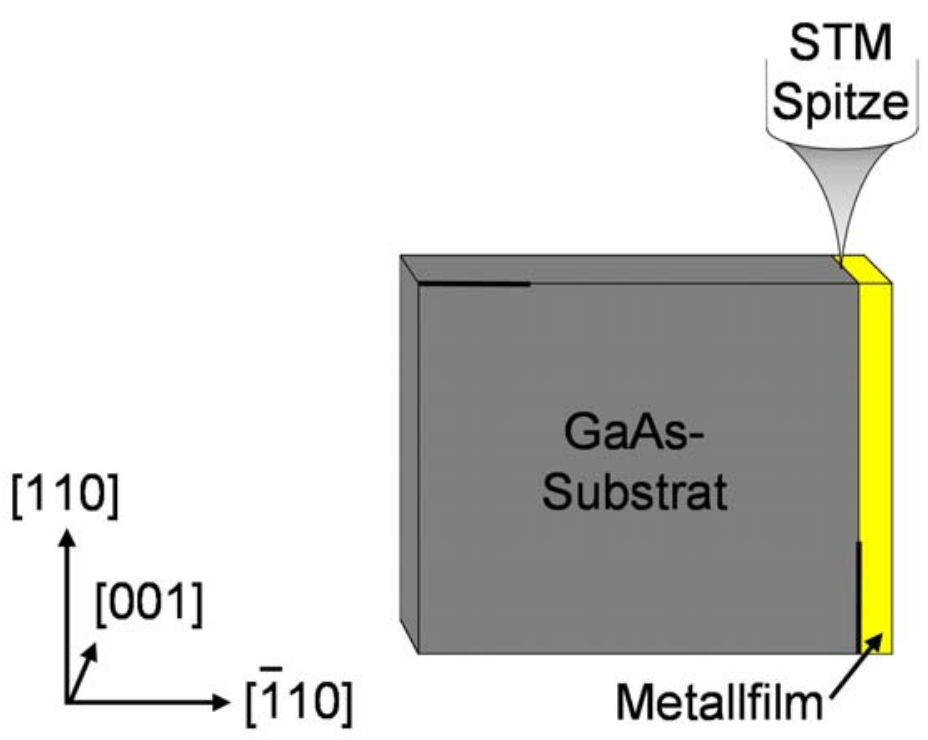

Abbildung 21: Messanordnung zur Untersuchung von Schottky-Kontakten in Querschnittsgeometrie. Auf der einen der orthogonal zueinander liegenden $\{110\}$ Spaltflächen wird der Metallfilm (gelb) aufgewachsen. Durch Spalten entlang der anderen natürlichen Bruchfläche wird die Metall-Halbleiter Grenzfläche mit dem STM zugänglich.

Als Basis diente ein (001)-orientierter n-GaAs Wafer. Wie in Abschnitt 3.2 geschildert können bei einem solchen Wafer zwei orthogonal zueinander liegende $\{110\}$ Spaltflächen präpariert werden. Zunächst wurde eine der Spaltflächen unter Hochvakuum-Bedingungen erzeugt. Bei RT wurde auf diese Spaltfläche ein etwa $10 \mathrm{~nm}$ dicker, polykristalliner Au-Film aufgewachsen. Um die Grenzfläche des so hergestellten Schottky-Kontakts für das STM zugänglich zu machen, wurde die Probe ein zweites Mal entlang der anderen natürlichen Bruchfläche gespalten. Mit dem STM konnten anschließend erstmals verschiedene Charakteristika des Schottky-Kontakts im Realraum auf der atomaren Skala untersucht werden:

- Sowohl spannungsabhängige Konstantstromtopographien als auch die räumlich aufgelöste Tunnelspektroskopie lassen Rückschlüsse auf die Defektdichte im Bereich der Grenzfläche zu.

- In Topographie und Spektroskopie ist der Einfluss der Raumladungszone deutlich zu erkennen.

- Es konnte ein signifikanter Einfluss von strukturellen Defekten auf die Potentiallandschaft in der RLZ beobachtet werden. Durch den Vergleich mit 1D-Tunnelstromsimulationen war es möglich, den Potentialverlauf in der Raumladungszone qualitativ nachzuvollziehen.

- In der Nähe der Grenzfläche zeigt die Tunnelspektroskopie Hinweise auf eine erhöhte Zustandsdichte im Energiebereich der GaAs Bandlücke.

- Defekte an der Metall-Halbleiter Grenzfläche konnten bei dem untersuchten Probensystem als dominierender Mechanismus des FLP ausgeschlossen werden. 
An dem Modellsystem konnte demonstriert werden, dass durch Spalten eines Schottky-Kontakts ein wohl definiertes System erzeugt werden kann, dass sich zur Untersuchung mit dem STM eignet. Darüber hinaus wurde gezeigt, dass durch diesen neuen Zugang sowohl Informationen über die strukturellen als auch über die elektronischen Eigenschaften eines Schottky-Kontakts gewonnen werden können. Abgesehen von den oben genannten Messungen sind keine weiteren XSTM Untersuchungen von Schottky-Kontakten bekannt.

Durch die ersten XSTM Messungen an dem Modellsystem wurde aber auch verdeutlicht, dass durch verschiedene Umstände sowohl die Untersuchung von Schottky-Kontakten mit dem XSTM als auch die Interpretation der Daten stark eingeschränkt werden kann. Dazu zählen:

- Aufgrund der Hochvakuum-Bedingungen, unter denen der Metallfilm deponiert wurde, kann ein signifikanter Einfluss von Restgasatomen auf die Ausbildung des Schottky-Kontakts nicht ausgeschlossen werden.

- Die Probe musste nach der Deposition des Metallfilms an Raumbedingungen für die XSTM Messungen vorbereitet werden. Dabei wurde die Probe unter anderem für einige Minuten einer Temperatur von über $160^{\circ} \mathrm{C}$ ausgesetzt. Um zu verhindern, dass der Bereich der Metall-Halbleiter Grenzfläche durch Oxidation verändert wird, wurde als Metallfilm ein relativ dicker Au-Film gewählt.

- Bei dem Spaltvorgang ist es nicht gelungen, im Bereich der Grenzfläche eine atomar glatte Oberfläche zu erzeugen. Der polykristalline Au-Film ist an den Korngrenzen des Films aufgebrochen, so dass weite Teile der Grenzfläche nicht mit dem STM zugänglich waren.

- Durch die fehlende atomare Auflösung war es nicht möglich, die vorhandenen Defekte im Bereich der Grenzfläche zu identifizieren. Es konnte daher nicht zwischen Defekten, die charakteristisch für den Schottky-Kontakt sind, und Defekten, die lediglich durch den Spaltvorgang hervorgerufen wurden, unterschieden werden. Grundsätzlich wird die strukturelle Charakterisierung der Grenzfläche auch dadurch erschwert, dass der Metallfilm des gewählten Modellsystems polykristallin ist, so dass sich die Komplexität der Struktur erhöht.

- Insbesondere die Unkenntnis des TIBB als Funktion des Ortes und der angelegten Bias-Spannung hat verhindert, dass die SBH sowie die Potentiallandschaft in der RLZ des Schottky-Kontakts durch den Vergleich von experimentellen mit simulierten Tunnelspektren quantitativ bestimmt werden konnten.

Im weiteren Verlauf der Arbeit werden daher zunächst die Strategien und Lösungswege beschrieben, die im Rahmen dieser Arbeit entwickelt wurden, um die XSTM Untersuchung von SchottkyKontakten zu optimieren, bevor in den Kapiteln 5, 6 und 7 auf die experimentellen Daten eingegangen wird. 


\section{Präparation von idealen Schottky-Kontakten zur Untersuchung mit dem XSTM}

Ideale Schottky-Kontakte bieten die besten Voraussetzungen, um Metall-Halbleiter Kontakte auf die fundamentalen Mechanismen hin zu untersuchen, die die Charakteristik ihrer jeweiligen, individuell unterschiedlichen Schottky-Barrieren bestimmen. Im Vergleich zu herkömmlichen Schottky-Kontakten ist die Komplexität von idealen Metall-Halbleiter Kontakten reduziert, so dass die Einflüsse der verschiedenen, in Kapitel 1 diskutierten Mechanismen besser voneinander getrennt werden können. Um derartige Probensysteme auf der Basis einer GaAs\{110\} Spaltfläche unter UHV Bedingungen herzustellen und anschießend in dem „as prepared“ Zustand mit dem STM in Querschnittsgeometrie detailliert untersuchen zu können, wurden verschiedene Aspekte der Probenpräparation grundlegend zu verändert.

Im ersten Abschnitt des Kapitels wird zunächst darauf eingegangen, welche Eigenschaften die GaAs\{110\} Spaltflächen als Substrat für das Wachstum von idealen Schottky-Kontakten auszeichnen und unter welchen Bedingungen diese Eigenschaften optimal genutzt werden können. Am Beispiel der Deposition eines Metallfilms auf einer GaAs(110) Spaltfläche wird in den Abschnitten 4.2 - 4.4 detailliert beschrieben, wie Schottky-Kontakte unter diesen optimalen Bedingungen derart präpariert werden können, dass sie anschließend mit dem STM in Querschnittsgeometrie untersucht werden können.

\subsection{Die GaAs $\{110\}$ Spaltflächen als Substrat für ideale Schottky-Kontakte}

Die GaAs $\{110\}$ Oberflächen sind seit langem beliebte Substrate, um die Ausbildung von SchottkyKontakten unter fundamentalen Gesichtspunkten zu untersuchen [61, 101-106]. Die Gründe dafür sind vielfältig:

- Das Fehlen eines intrinsischen Oberflächenzustands im Energiebereich der fundamentalen Bandlücke ist ein wichtiges Charakteristikum der GaAs\{110\} Spaltflächen [89], das sie als Substrat für die Untersuchung der grundlegenden Mechanismen der Schottky-Barrierenformierung besonders interessant machen. In Kapitel 1 wurde die Rolle von Bandlückenzuständen an der MetallHalbleiter Grenzfläche im Rahmen der verschiedenen gängigen Theorien diskutiert. Sie sind das zentrale Element, dass für die wesentlich schwächere Abhängigkeit der SBH von der Austrittsarbeit der Metalle verantwortlich gemacht wird, als von der Schottky-Mott-Theorie vorhergesagt. Da verschiedene Ursachen für die Existenz von Bandlückenzuständen in Frage kommen, kann 
durch die Wahl einer GaAs\{110\} Spaltfläche ohne intrinsischen Oberflächenzustand die Komplexität der Schottky-Barrierenformierung reduziert werden.

- Die GaAs\{110\} Spaltflächen sind unrekonstruiert [88]. Die Existenz von verschiedenen Oberflächenrekonstruktionen, die häufig nebeneinander existieren können, erhöht die Komplexität einer Metall-Halbleiter Grenzfläche und ist von daher unerwünscht.

- Die GaAs\{110\} Spaltflächen lassen sich relativ einfach erzeugen, da es sich um die natürlichen Bruchflächen des Kristalls handelt. Unter Verwendung geeigneter Spaltverfahren gelingt es, die Anzahl von Oberflächendefekten wie Substratstufen und Fehlstellen auf ein Minimum zu reduzieren [87]. Der Einfluss dieser Defekte auf die Ausbildung der Schottky-Barriere oder das Wachstum des Metallfilms kann in diesen Fällen ausgeschlossen werden [102]. Wird der Spaltvorgang darüber hinaus im UHV durchgeführt, so kann man eine nahezu perfekte, planare Substratoberfläche ohne jegliche Kontamination generieren.

Die GaAs\{110\} Spaltflächen sind daher unter dem Gesichtspunkt einer möglichst homogenen und einfachen atomaren und elektronischen Struktur ein optimales Substrat zum Wachstum von idealen Schottky-Kontakten im Sinne von Kapitel 1.1.

Neben den Eigenschaften der Substratoberfläche bestimmen vor allem die Bedingungen während der Deposition des Metallfilms die Eigenschaften eines Schottky-Kontakts. Durch die Bedingungen muss gewährleistet werden, dass die folgenden Kriterien bei dem Wachstum des Metallfilms erfüllt werden:

- Reaktionen an der Metall-Halbleiter Grenzfläche müssen unterdrückt werden, so dass es zur Ausbildung einer homogenen, abrupten Grenzfläche kommt.

- Epitaktisches Wachstum eines möglichst defektfreien, einkristallinen Films.

Das epitaktische Wachstum von diversen Metallfilmen auf GaAs\{110\} Oberflächen wurde bereits in verschiedenen Arbeiten intensiv untersucht [107, 108]. Üblicherweise kommt es zur Ausbildung eines durchmischten Grenzflächenbereichs, wobei das Ausmaß der Reaktionen an der Grenzfläche von dem jeweiligen Metall abhängt. Es konnte gezeigt werden, dass durch ein Absenken der Substrattemperatur während der Deposition der Grad der Durchmischung an der Grenzfläche reduziert werden kann [109]. Auf der anderen Seite führt eine niedrige Substrattemperatur aufgrund der verringerten Mobilität der deponierten Metallatome in der Regel zu einer erhöhten Defektdichte im Metallfilm [42, 110], so dass beide oben genannten Kriterien durch das alleinige Absenken der Substrattemperatur nicht gleichzeitig erreicht werden können.

Erst durch die Kombination der Tieftemperaturdeposition des Metallfilms mit einem anschließenden Annealing ist es im Fall von Ag-Filmen gelungen, sowohl eine abrupte Grenzfläche als auch einen einkristallinen Metallfilm auf einer GaAs(110) Spaltfläche zu präparieren [111]. Bei diesem in zwei Schritten ablaufenden Wachstumsprozess wurde zunächst ein nur wenige Monolagen dicker Ag-Film 
bei $135 \mathrm{~K}$ deponiert. Bei $145 \mathrm{~K}$ durchgeführte STM Messungen haben gezeigt, dass der Film geschlossen ist und aus Nanoclustern besteht. Anschließend wurde der Film im zweiten Präparationsschritt bis auf RT annealt. Während dieses Prozesses ist es zu einer Umordnung des gesamten Films gekommen und es hat sich ein atomar flacher, einkristalliner Ag-Film formiert. An einigen Stellen war der Ag-Film nicht mehr geschlossen, so dass die GaAs(110) Oberfläche mit dem STM abgebildet werden konnte. Da in diesen Bereichen keine strukturellen Veränderungen der Substratoberfläche beobachtet werden konnten ist davon auszugehen, dass es bei dem Wachstumsprozess zu keiner Durchmischung an der Metall-Halbleiter Grenzfläche gekommen ist. Ein ähnliches Wachstumsverhalten konnte auch bei Cu-Filmen beobachtet werden, die nach dem beschriebenen Schema präpariert worden sind [112]. Diese Ergebnisse deuten an, dass die GaAs\{110\} Spaltflächen erst in Verbindung mit dem aus Tieftemperaturdeposition und anschließendem Annealing bestehenden Wachstumsprozess als Substrat zur Herstellung von idealen Schottky-Kontakten genutzt werden können.

\subsection{Vorbereitung des GaAs Substrats für die UHV Präparation}

Der Probenpräparation kommt im Rahmen dieser Arbeit eine wichtige Rolle zu, um zum einen ideale Schottky-Kontakte herstellen zu können, und um zum anderen den Zugang mit dem STM in Querschnittsgeometrie zu gewährleisten.

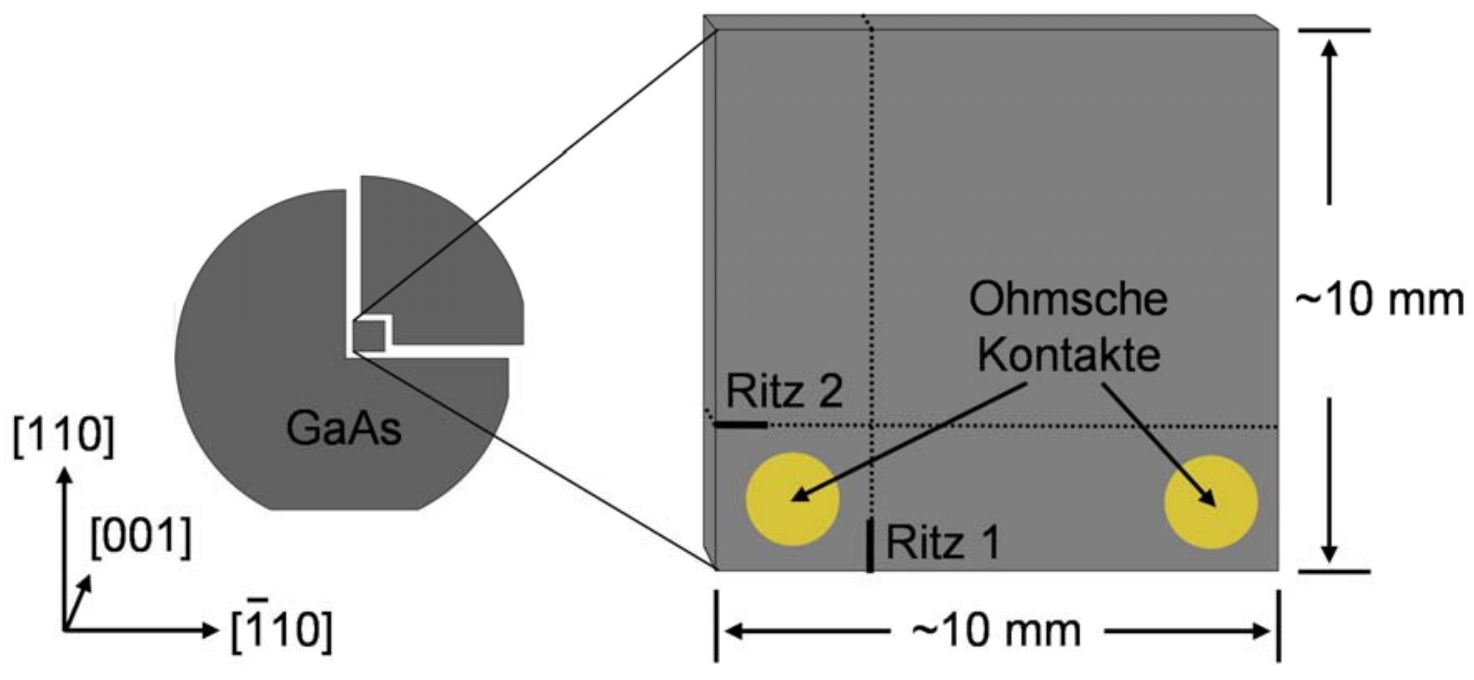

Abbildung 22: Prozessschritte vor dem Einschleusen in die UHV Präparationskammer. Durch die Ritze können unter UHV Bedingungen zwei orthogonal zueinander liegende Spaltflächen (angedeutet durch die gepunkteten Linien) nacheinander erzeugt werden. Für die STM Messung befinden sich zwei Ohmsche Kontakte in den Ecken der Probe. 
In Abbildung 22 sind die Prozessschritte zusammengefasst, die vor dem Einbau des GaAs Substrats in den Probenhalter durchgeführt werden müssen.

- Unter Ausnutzung der natürlichen Spaltflächen des GaAs Kristalls wird aus dem (001)-orientierten GaAs Wafer ein etwa 10 x $10 \mathrm{~mm}^{2}$ großes Element heraus gebrochen.

- Die Probe wird bis auf eine Dicke von etwa $100 \mu \mathrm{m}$ gedünnt. Erfahrungsgemäß sollte die Probe möglichst dünn sein, um beim Spalten im UHV möglichst perfekte Bruchflächen mit einer geringen Defektdichte zu erhalten [87]. Unterhalb einer Dicke von etwa $100 \mu \mathrm{m}$ wird die Probe allerdings für die folgenden Prozessschritte zu fragil.

- In zwei Ecken der Probe werden tieftemperaturtaugliche Ohmsche Kontakte durch Aufdampfen und anschließendes Annealen präpariert ${ }^{10}$, so dass die Probe für die STM Messung kontaktiert werden kann.

- Die Probe wird an zwei Stellen mit orthogonal zueinander verlaufenden Ritzen versehen. Diese beiden Ritze sind essentiell, um die zwei nacheinander im UHV durchzuführenden Spaltvorgänge kontrollieren zu können.

Durch die Initialritze wird die Struktur der Probe an den entsprechenden Stellen beschädigt. Da eine mechanisch belastete Probe bevorzugt an den Stellen spalten wird, an denen die Struktur bereits geschwächt ist, kann durch die Ritze beeinflusst werden, wo die Bruchflächen im UHV erzeugt werden [73]. Zur Präparation der Ritze wird eine Wolfram-Carbid Nadel mit einem Krümmungsradius von etwa $10 \mu \mathrm{m}$ verwendet, die mit einer Kraft von typischerweise $20 \mathrm{~g}$ auf der (001) Oberfläche der Probe aufliegt. Die Nadel wird nun in einer geraden Linie etwa $500 \mu \mathrm{m}$ weit senkrecht zu der Normalen der gewünschten Bruchfläche über die Probe bewegt. Dadurch wird im Bereich des Ritzes sowohl die Oberfläche lokal beschädigt als auch ein ausgedehntes Versetzungsnetzwerk in der zu präparierenden Bruchfläche erzeugt [87]. Eine der Schwierigkeiten bestand darin, dass die Probe bereits nach dem Anbringen des ersten Ritzes extrem fragil wird und schon durch geringe mechanische Belastungen, wie z.B. bei dem Präparieren des zweiten Ritzes, der Spaltvorgang ausgelöst werden kann. Durch die Anpassung der Auflagekraft der Ritznadel an die jeweilige Dicke der gedünnten Probe konnte dieses Problem beseitigt werden.

Die zweifach geritzte Probe wird im nächsten Schritt in einen speziell angefertigten Probenhalter eingebaut (Abbildung 23). In dem Probenhalter wird lediglich die linke untere Ecke der Probe fixiert,

\footnotetext{
${ }^{10}$ Im Fall von n-GaAs Substraten, die in dieser Arbeit verwendet wurden, wurde als Depositmaterial ein AuGe-Eutektikum verwendet. Details zur Präparation der Ohmschen Kontakte auf n-GaAs finden sich z.B. in [100].
} 
indem sie durch eine Klemmschraube und eine CuBe-Feder leicht gegen die feststehende $\mathrm{Cu}$ Klemmbacke gedrückt wird.

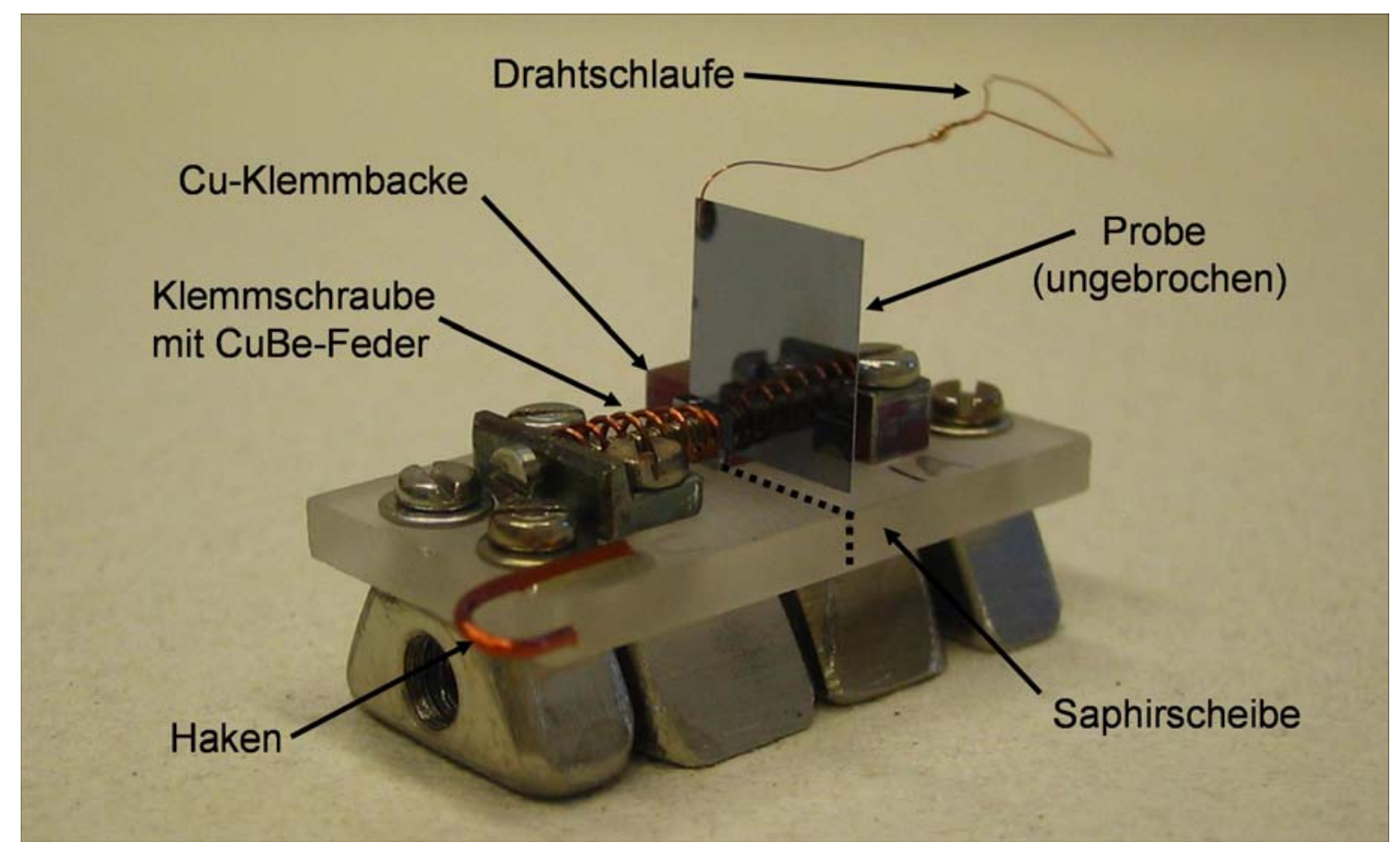

Abbildung 23: Fotografie eines 4-fach segmentierten Probenhalters mit eingebauter Probe vor dem Einschleusen in die UHV Präparationskammer. Die zweifach geritzte Probe wird durch eine Klemmschraube und eine CuBe-Feder gegen die feststehende Cu-Klemmbacke gedrückt. Um den zweiten Spaltvorgang direkt im STM bei 6 K durchführen zu können, befindet sich an der Probe eine Drahtschlaufe und an dem Probenhalter ein Haken. Eine detaillierte Beschreibung findet sich in Kapitel 4.4 .

Um die Probe optimal im UHV spalten zu können, müssen bei der Positionierung der Probe relativ zu der feststehenden Klemmbacke folgende Punkte beachtet werden:

- Beide Ritze dürfen sich nicht im Bereich der Klemmbacke befinden, da ansonsten stark gestufte, defektreiche und damit unbrauchbare Bruchflächen entstehen.

- Die Ritze sollten sich möglichst dicht an den Kanten der Cu-Klemmbacke befinden, da so die besten Spaltflächen erzeugt werden können.

- Die Spaltflächen müssen komplett über bzw. neben der Klemmbacke verlaufen. Die GaAs\{110\} Richtungen müssen dafür möglichst parallel zu den Kanten der Klemmbacke ausgerichtet werden.

- Das GaAs Substrat darf die Saphirscheibe nicht berühren.

- Die rechte untere Ecke und die linke obere Ecke müssen frei zugänglich sein, da auf die Probe an diesen Stellen im UHV eine Kraft ausgeübt werden muss, um den jeweiligen Spaltvorgang auszulösen (siehe Kapitel 4.3 und 4.4). 
Um sicherzustellen, dass diese Kriterien erfüllt werden, wurde zum Einbau der Proben eine Stereolupe verwendet.

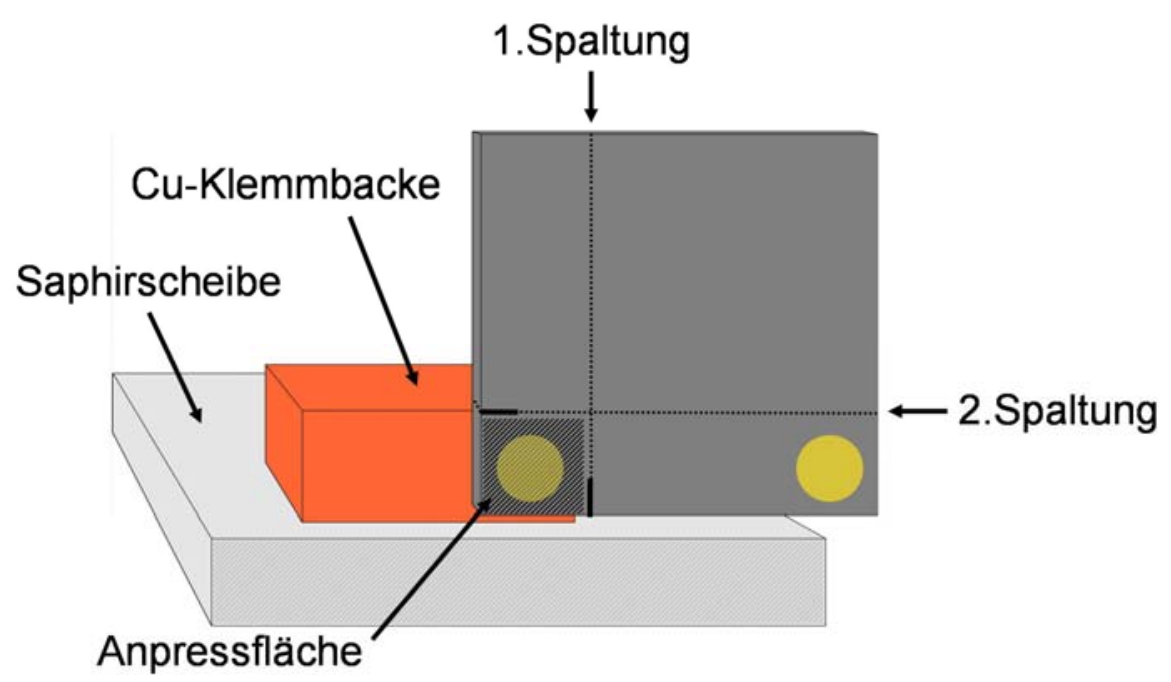

Abbildung 24: Schnitt durch den Probenhalter mit optimal eingebauter Probe. Aus Übersichtsgründen sind lediglich Saphirscheibe, Cu-Klemmbacke und zweifach geritzte Probe dargestellt. Die Ebenen, in denen die erste bzw. zweite Spaltfläche erzeugt werden, sind durch die gepunkteten Linien angedeutet.

In Abbildung 24 ist ein Schnitt durch den Probenhalter mit optimal eingebauter Probe skizziert (die Position des Schnitts wird in Abbildung 23 durch die gestrichelte Linie angedeutet). Der Ritz für den ersten Spaltvorgang liegt etwa $100 \mu \mathrm{m}$ rechts von der Kante der Klemmbacke, der Ritz für den zweiten Spaltvorgang befindet sich etwa $100 \mu$ m über der oberen Kante der Klemmbacke. Die untere Kante der Probe befindet sich etwa $500 \mu \mathrm{m}$ über der Saphirscheibe.

Durch die geschilderte Vorbereitung des GaAs Substrats und den speziellen Einbau in den Probenhalter können alle weitern Präparationsschritte bis hin zur Untersuchung des Schottky-Kontakts in Querschnittsgeometrie unter UHV Bedingungen durchgeführt werden.

\subsection{UHV Präparation des Schottky-Kontakts auf der GaAs(110) Spaltfläche bei tiefen Temperaturen}

Ideale Metall-Halbleiter Kontakte zeichnen sich durch eine homogene, abrupte Grenzfläche zwischen dem einkristallinen Halbleiter Substrat und dem epitaktisch aufgewachsenen einkristallinen Metallfilm aus. Eine Grundvoraussetzung, um dies zu erreichen, ist sowohl eine saubere und defektfreie Substratoberfläche als auch die Vermeidung jeglicher Kontamination während der Deposition des Metallfilms, die zu neuen chemischen, elektronischen, und morphologischen Eigenschaften führen kann [24]. Zur Präparation idealer Schottky-Kontakte kommen daher ausschließlich UHV Methoden in Frage. Der im 
Folgenden geschilderte UHV Präparationszyklus entspricht in den Grundzügen der von Smith et al. publizierten Präparation dünner, einkristalliner Ag-Filme auf der GaAs(110) Spaltfläche [111]:

- Deposition des Metallfilms bei $135 \mathrm{~K}$ auf einer GaAs $\{110\}$ Spaltfläche

- Langsames Erwärmen der Probe zu RT
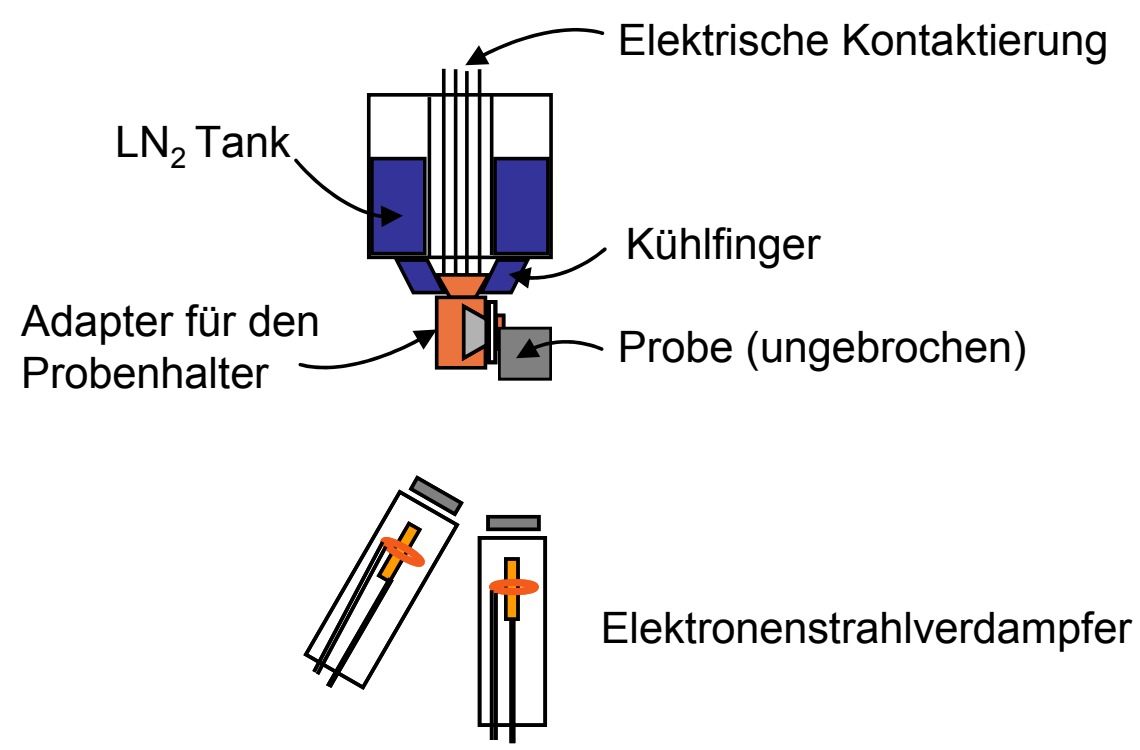

Abbildung 25: Skizze der UHV Präparationskammer. Dargestellt sind sowohl zwei Elektronenstrahlverdampfer als auch die Probenaufnahme. Zur Deposition des Schottky-Kontakts auf der ersten Spaltfläche muss ein Adapter verwendet werden, durch den der Probenhalter um 90 Grad gedreht wird.

Die in dieser Arbeit verwendete UHV Präparationskammer wurde bereits in verschiedenen anderen Arbeiten im Detail beschrieben, so dass hier nur kurz auf die wesentlichen Charakteristika eingegangen wird [113, 114]. Abbildung 25 zeigt eine Skizze der UHV Präparationskammer. Die UHV Kammer arbeitet bei einem Basisdruck von unter $5 \mathrm{e}^{-11}$ mbar und kann direkt mit der STM Kammer verbunden werden, in der die spätere Untersuchung in Querschnittsgeometrie stattfindet. Die Präparationskammer ist unter anderem mit zwei flussstabilisierten Elektronenstrahlverdampfern der Firma Tectra ausgerüstet, die mit verschiedenen Depositmaterialien bestückt werden können. Zur Präparation der in den folgenden Kapiteln diskutierten Proben wurde der zentrale Verdampfer mit einem Fe-Stab ${ }^{11}$ ausgerüstet, in dem zweiten Verdampfer befand sich ein Molybdän-Tiegel mit Bi ${ }^{12}$. Die Flüsse der beiden Verdampfer wurden im Laufe dieser Arbeit mehrfach geeicht, indem bei den später verwendeten Setzparametern Filme von etwa $30 \mathrm{~nm}$ Dicke auf GaAs Spaltflächen deponiert wurden. Die tatsächlichen Dicken der Filme wurden anschließend mit einem AFM bestimmt. Kontinuierliche

\footnotetext{
${ }^{11}$ Alfa Aesar, puratronic, 99,995 \% (metals basis)

${ }^{12}$ Alfa Aesar, 99,997 \% (metals basis)
} 
Veränderungen in den Charakteristiken der Verdampfer sorgen dafür, dass die Abweichungen von den in den Kapiteln 5 und 6 angegeben Schichtdicken bis zu 10 \% betragen können. Über den Verdampfern befindet sich die Probenaufnahme, deren Position relativ zu den Verdampfern über Mikrometerschrauben verändert werden kann. In der Probenaufnahme kann der Probenhalter elektrisch kontaktiert werden und bei Bedarf geheizt bzw. heruntergekühlt werden. Zur Kühlung der Probe wird die Probenaufnahme, die thermisch an ein $\mathrm{LN}_{2}$ Tank angekoppelt ist, als Kühlfinger verwendet. In Abhängigkeit von dem thermischen Kontakt zwischen dem jeweiligen Probenhalter und der Probenaufnahme kann die Probe bis auf 130(5) K heruntergekühlt werden. Um den Schottky-Kontakt auf der ersten Spaltfläche der Probe präparieren zu können, muss ein Adapter verwendet werden, so dass der Probenhalter um 90 Grad gedreht eingebaut werden kann.

Für den weiteren Verlauf der Probenpräparation ist es essentiell, gezielt nur den ersten der beiden Spaltvorgänge zur Präparation der Substratoberfläche auszulösen. Dabei sind sowohl der Angriffspunkt als auch die Richtung der Kraft entscheidend, die dazu auf die Probe ausgeübt wird. Wie in Abbildung 26 (a) skizziert darf eine Kraft ausschließlich in der durch den roten Pfeil markierten Ecke senkrecht auf die (001) Oberfläche der Probe ausgeübt werden, um eine optimale erste Spaltfläche erzeugen zu können. Wird die Probe an anderen Stellen belastet, kann es zur Auslösung beider Spaltvorgänge kommen und es entstehen in der Regel stark gestufte Spaltflächen, die als Substrat zum Wachstum von idealen Schottky-Kontakten ungeeignet sind.

(a)

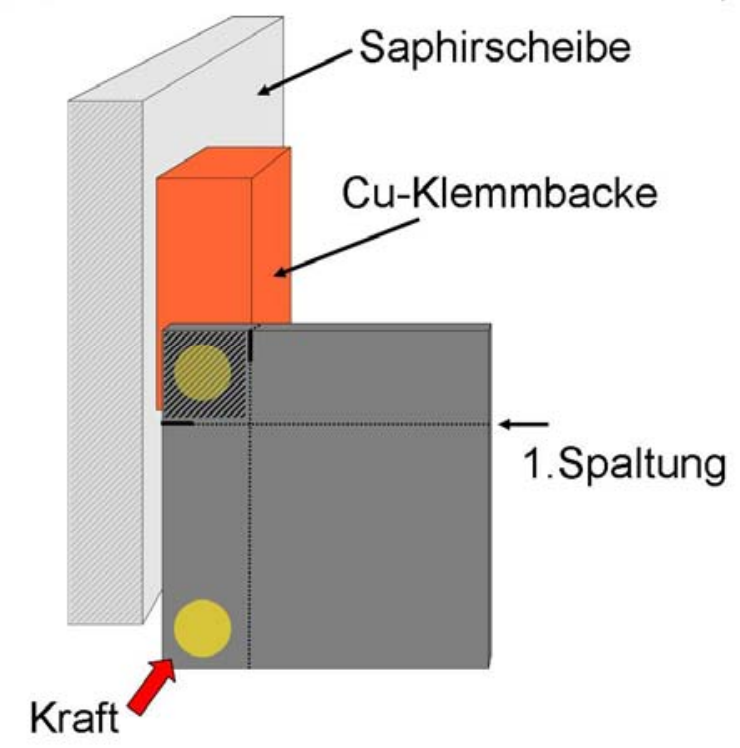

(b)

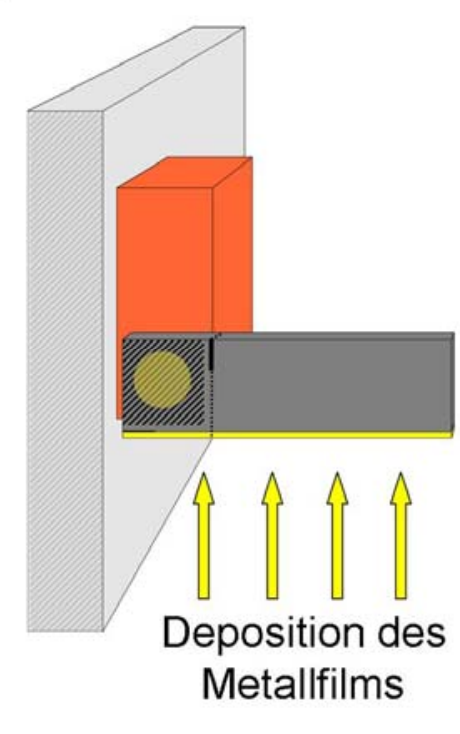

Abbildung 26: Zur Präparation des Schottky-Kontakts wird der Probenhalter um 90 Grad gedreht eingebaut und auf etwa 130(5) K heruntergekühlt. (a) Der erste Spaltvorgang wird ausgelöst, indem eine Kraft ausschließlich auf die rechte untere Ecke der Probe ausgeübt wird. (b) Direkt nach dem Spaltvorgang wird der Metallfilm auf die saubere Spaltfläche deponiert. 
Zum Zeitpunkt des ersten Spaltvorgangs befindet sich die Probe bereits bei 130(5) K, so dass direkt im Anschluss mit der Deposition des Metallfilms auf die saubere GaAs(110) Spaltfläche begonnen werden kann (Abbildung 26 (b)). Durch diesen Ablauf wird zum einen verhindert, dass es durch das Restgas zu einer Kontamination der frischen Spaltfläche kommt. Zum anderen wird vermieden, dass es nach dem Spaltvorgang durch Langmuir-Desorption zur Ausbildung von Oberflächendefekten wie Fehlstellen und Adatomen kommt [63]. Zur Deposition der in den folgenden Kapiteln diskutierten Fe- und Bi-Filme wurden Flüsse von etwa 1 ML / Minute verwendet. Während der Deposition steigt der Druck in der UHV Kammer nicht über $5 \mathrm{e}^{-10} \mathrm{mbar}$, in der Regel bleibt er unter $3 \mathrm{e}^{-10}$ mbar. Nach der Deposition des Metallfilms wird der Probenhalter aus der Probenaufnahme ausgebaut und erwärmt sich langsam innerhalb von 2 Stunden an einem UHV Manipulator zu RT. Direkt im Anschluss wird die Probe unter UHV Bedingungen in die angeschlossene STM Kammer transferiert. Da der Initialritz zur Erzeugung der orthogonalen zweiten Spaltfläche für die XSTM Messung bereits vorhanden ist, muss die Probe nicht mehr zwischen den beiden Spaltvorgängen ex-situ präpariert werden.

\subsection{Spalten des Schottky-Kontakts bei $6 \mathrm{~K}$}

Direkt vor der Messung mit dem STM in Querschnittsgeometrie (Abbildung 27 (b)) muss die Probe ein zweites Mal in der STM Kammer über die Grenzfläche des präparierten Schottky-Kontakts hinweg gespalten werden. Dieser Spaltvorgang ist von daher besonders heikel, da die Qualität der erzeugten Bruchfläche darüber entscheidet, wie gut sich die Probe für die Untersuchung mit dem XSTM eignet. Im Idealfall sollte durch den Spaltvorgang eine atomar glatte, defektfreie Oberfläche über die MetallHalbleiter Grenzfläche hinweg erzeugt werden.

(a)

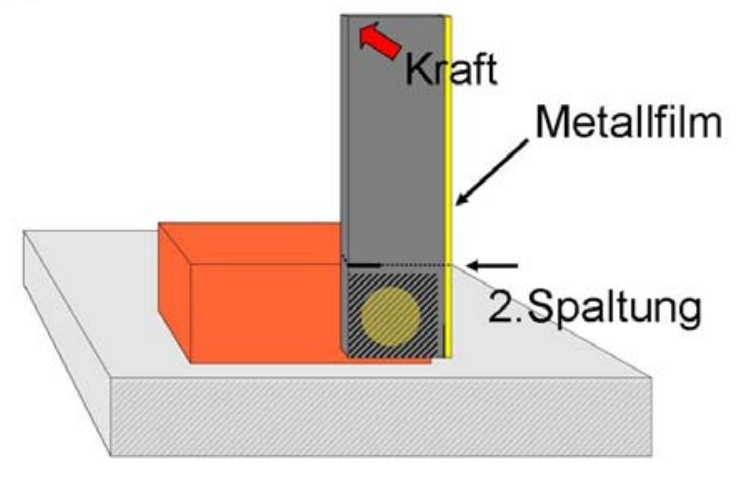

(b)

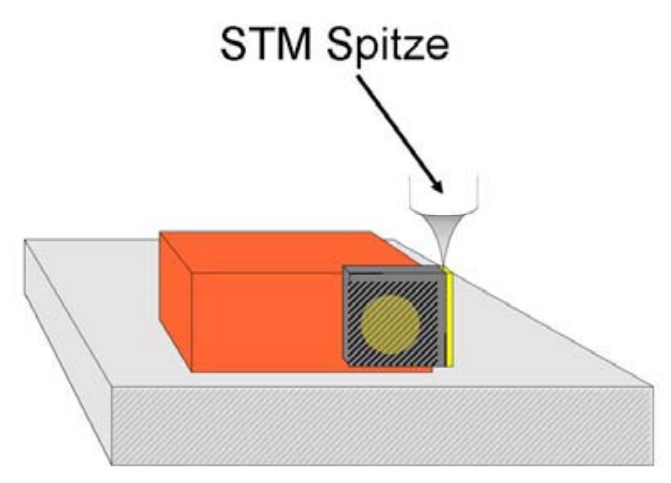

Abbildung 27: (a) Durch das Ausüben einer Kraft in der oberen linken Ecke der Probe wird der zweite Spaltvorgang ausgelöst. (b) Die Grenzfläche des Schottky-Kontakts kann mit dem XSTM auf der atomaren Skala untersucht werden. 
Um den Spaltvorgang auszulösen, wird die Probe wie in Abbildung 27 (a) gezeigt in der linken oberen Ecke belastet. Die im Vorfeld dieser Arbeit durchgeführten ersten Spaltversuche von MetallHalbleiter Kontakten (siehe Kapitel 3.4) haben gezeigt, dass das Spaltverhalten des GaAs Substrats durch den Metallfilm nur geringfügig beeinflusst wird [93]. Abbildung 28 zeigt eine AFM Topografie der Spaltfläche des Au-GaAs(110) Schottky-Kontakts. Bei der richtigen Anordnung von Initialritz und Belastung der Probe kommt es im Bereich des Substrats zu einem Sprödbruch des GaAs Kristalls und es wird eine $\{110\}$ Oberfläche erzeugt, die sich zur Untersuchung mit dem XSTM eignet. Verspannungen durch die Gitterfehlanpassung zwischen Substrat und Depositmaterial können allerdings dazu führen, dass vermehrt monoatomare Stufen im Bereich der GaAs Spaltfläche auftreten, die die STM Messungen beeinträchtigen. ${ }^{13}$

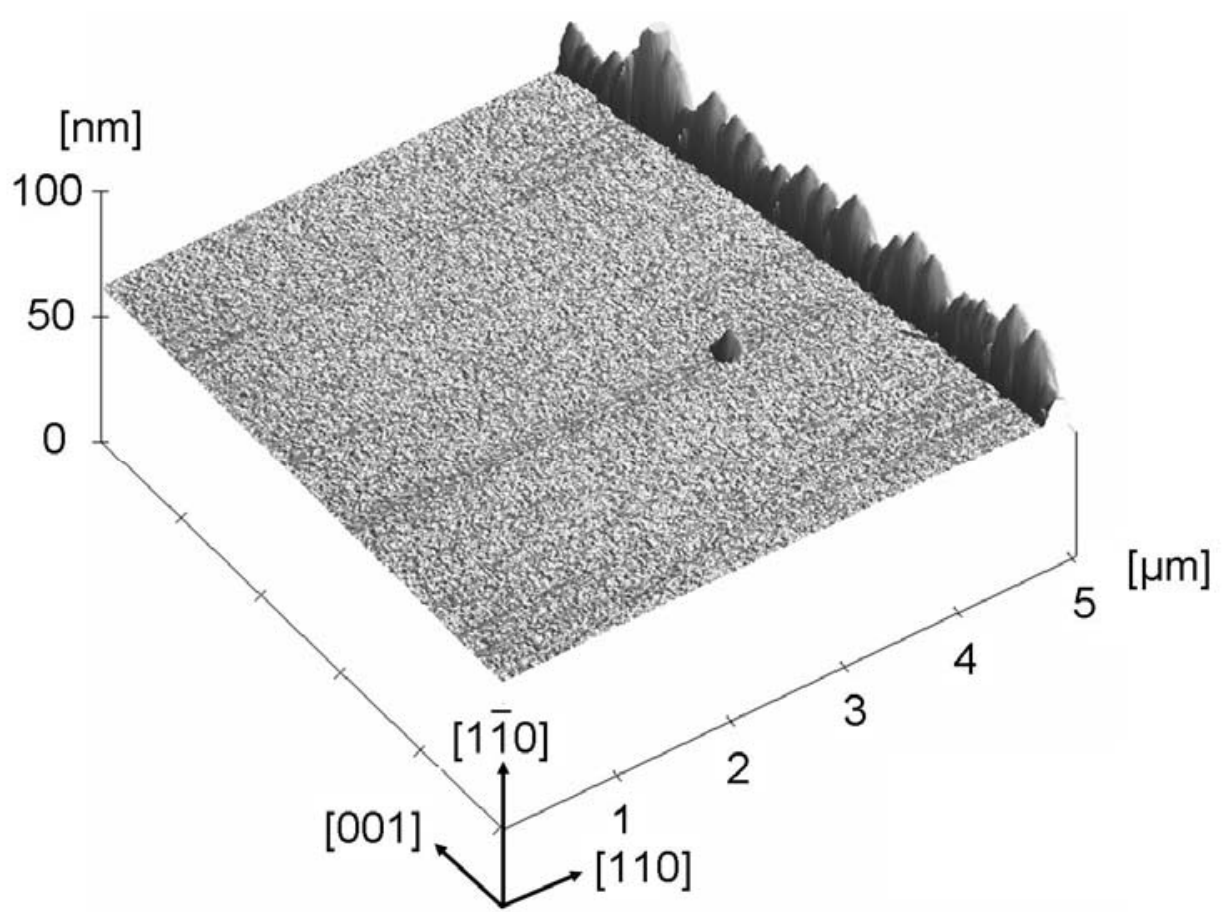

Abbildung 28: AFM Topografie der Au-GaAs(110) Spaltfläche. Das GaAs Substrat ist trotz des Metallfilms atomar glatt gespalten. Bei dem duktilen Au-Film ist es zu einem Verformungsbruch gekommen, so dass der Metallfilm über die Spaltebene des Substrats hinausragt.

Im Gegensatz zu dem Substrat konnte im Bereich des Metallfilms keine geeignete Oberfläche erzeugt werden. Die Morphologie des gespaltenen Au-Films deutet darauf hin, dass es bei dem Metallfilm zu einem Verformungsbruch gekommen ist, der für duktile Materialien typisch ist. Charakteristisch für diesen Bruch ist eine starke Einschnürung im Bereich der Bruchzone, da es zunächst zu einer größeren

\footnotetext{
${ }^{13}$ Aus Untersuchungen von Halbleiter-Heterostrukturen mit dem XSTM ist bekannt, dass bereits leichte Verspannungen des Kristalls im Bereich der Heterostruktur zu stark gestuften Oberflächen führen können [80-83].
} 
plastischen Deformation kommt, bevor das Material bricht. Die Struktur des Metallfilms wird durch den Spaltvorgang verändert und lässt daher keine Rückschlüsse auf die vor dem Spaltvorgang im Volumen der Probe herrschende Situation zu. Oberflächen, die durch einen Verformungsbruch erzeugt werden, sind daher für XSTM Untersuchungen ungeeignet. Darüber hinaus ragt der Metallfilm als Folge der plastischen Deformation entlang der Metall-Halbleiter Grenzfläche nahezu überall weit über die GaAs Spaltebene hinaus. Die entsprechende Situation und ihre Auswirkung auf die Untersuchung mit dem STM ist in Abbildung 29 skizziert.

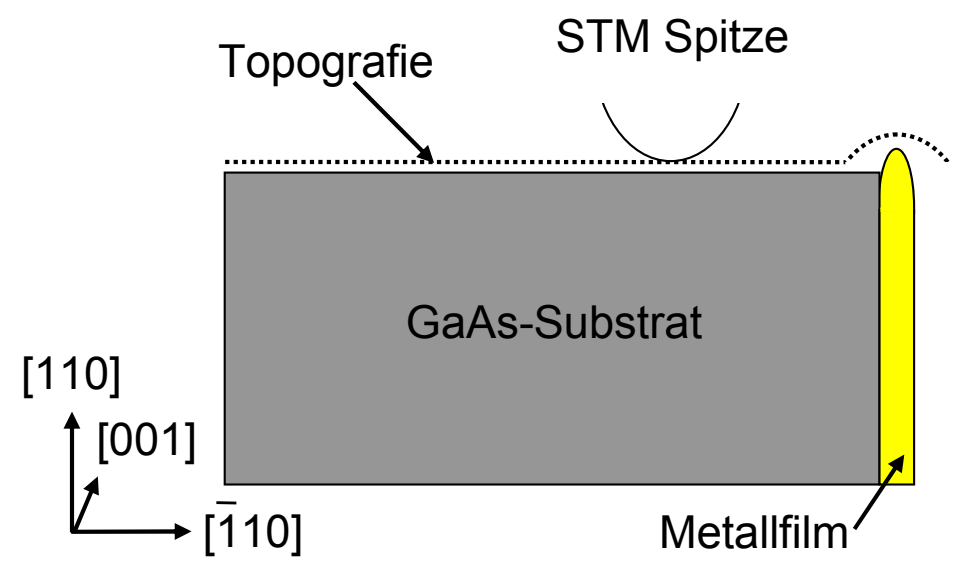

Abbildung 29: STM Messung eines nicht ideal gespaltenen Schottky-Kontakts. Durch die Ausdehnung der STM Spitze kann die Grenzfläche in Bereichen, in denen der Metallfilm über die Ebene der GaAs Spaltfläche hinausragt, nicht abgebildet werden. Die gepunktete Linie deutet die mit dem STM gemessene Topografie an.

Da es im Fall des Au-GaAs(110) Schottky-Kontakts nicht gelungen ist, das System in einer Ebene zu spalten, konnte auch das atomar glatte gespaltene GaAs Substrats nicht optimal mit dem STM untersucht werden. Wie in Abbildung 29 durch die gepunktete Linie angedeutet, ist es durch die Ausdehnung der STM Spitze nicht möglich, den aus der Sicht der Schottky-Physik besonders interessanten Bereich der Grenzfläche abzubilden. ${ }^{14}$

Um die Möglichkeiten, die die Untersuchung von Schottky-Kontakten mit dem XSTM prinzipiell bietet, besser ausnutzen zu können, war es daher entscheidend, vor allem das Spaltverhalten des Metallfilms zu optimieren. Eine deutliche Verbesserung konnte zum einen dadurch erreicht werden, dass die Dicke der im UHV deponierten Metallfilme durch die veränderten Abläufe während der Pro-

\footnotetext{
${ }^{14}$ STM Topografien vermitteln den Eindruck, dass der Metallfilm an diesen Stellen in den Bereich des GaAs Substrats hineinragt. Es handelt sich hierbei um ein Abbildungsartefakt, dass durch die Ausdehnung der Spitze hervorgerufen wird. In Kapitel 6.2 wird dieses Artefakt genutzt, um geometrische
} Eigenschaften der Tunnelspitze zu bestimmen. 
benpräparation reduziert werden konnte. Nach dem zweiten Spaltvorgang ragt der Metallfilm nur noch geringfügig über die Spaltebene des Substrats hinaus, so dass die GaAs Spaltfläche an vielen Stellen bis hin zur Metall-Halbleiter Grenzfläche mit dem STM zugänglich ist (Kapitel 6.1). Der Verformungsbruch des Metallfilms verhindert aber weiterhin, dass Informationen über die Struktur des Metallfilms vor dem Spaltvorgang gewonnen werden können. Um auch dies zu ermöglichen, muss es gelingen, sowohl das GaAs Substrat als auch den Metallfilm spröde zu spalten. Bei duktilen Metallen können Sprödbrüche z.B. bei sehr tiefen Temperaturen oder bei sehr hohen Verformungsgeschwindigkeiten vorkommen. Daher wurde eine Technik entwickelt, die es ermöglicht, die Probe vor dem zweiten Spaltvorgang auf $6 \mathrm{~K}$ abzukühlen. An der zweifach geritzten Probe wurde dazu vor dem Einschleusen in die UHV Präparationskammer eine Drahtschlaufe befestigt (siehe Abbildung 23). Der Angriffspunkt des Drahtes entspricht der Position, an der bei dem zweiten Spaltvorgang die Belastung der Probe stattfinden muss. Der Schottky-Kontakt wird wie in Kapitel 4.3 beschrieben präpariert. Nach der Präparation wird der Probenhalter in das Tieftemperatur XSTM eingebaut, so dass die Probe auf die Arbeitstemperatur von $6 \mathrm{~K}$ abgekühlt wird. Da sich an jedem Probenhalter (siehe Abbildung 23) ein Haken befindet, kann ein zweiter Probenhalter auf den UHV Manipulator aufgenommen werden, um an der Drahtschlaufe zu ziehen und so den zweiten Spaltvorgang bei 6 K auszulösen.

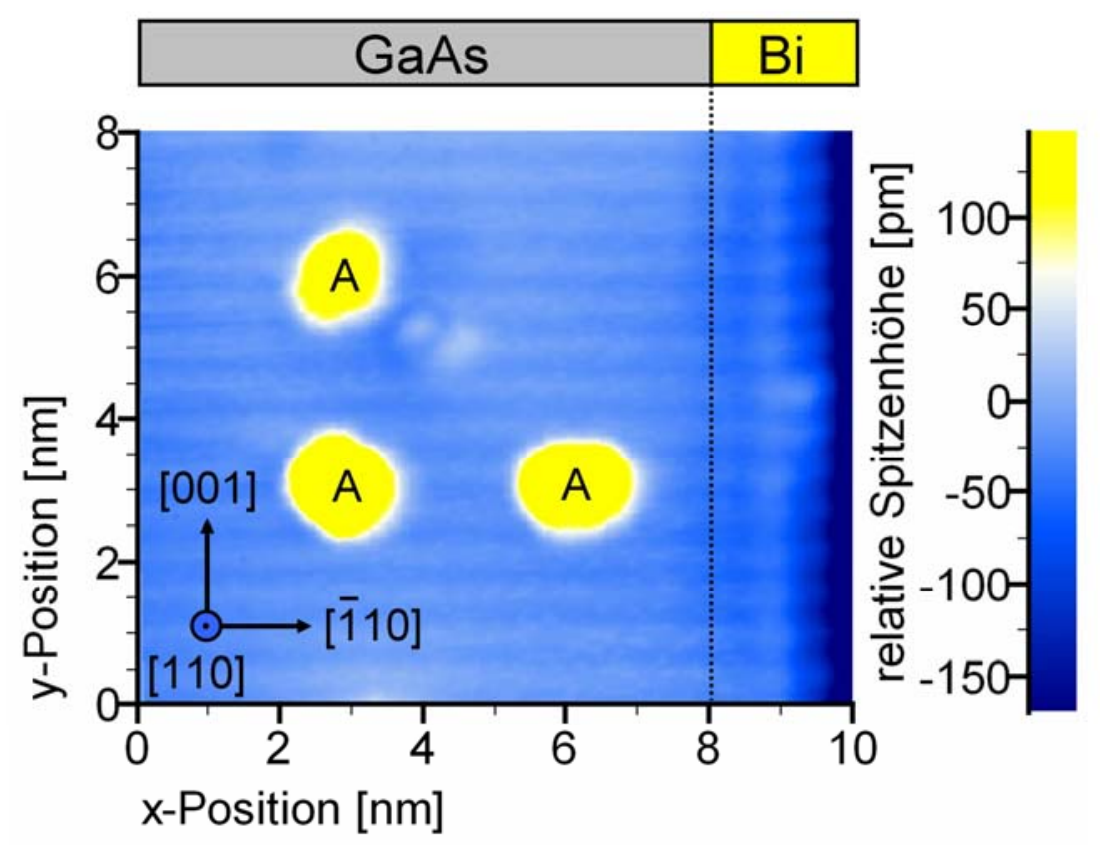

Abbildung 30: XSTM Topografie eines Bi-GaAs(1110) Schottky-Kontakts. Der zweite Spaltvorgang wurde bei $6 \mathrm{~K}$ durchgeführt. Bei den Erhöhungen auf der ansonsten atomar glatten Oberfläche handelt es sich um einzelne Adatome (A).

Abbildung 30 zeigt am Beispiel eines Bi-GaAs(110) Schottky-Kontakts, dass es durch dieses Verfahren gelungen ist, eine atomar glatte Spaltfläche über die Grenzfläche hinweg zu erzeugen. In der 
XSTM Topografie kann der Bereich des Bi-Films lediglich durch eine geringe Änderung des Kontrasts von nur wenigen pm von dem Bereich des Substrats unterschieden werden. Sowohl das GaAs Substrat als auch der $22 \AA$ dicke epitaktische Bi-Film konnten in derselben Ebene gespalten werden. Eine derart präparierte Probenoberfläche bietet im Gegensatz zu den in Kapitel 3.4 beschriebenen Spaltflächen ideale Voraussetzungen, um das Potential des STMs zur Untersuchung von Schottky-Kontakten in Querschnittsgeometrie voll ausnutzen zu können. 


\section{Der Fe-GaAs $\{110\}$ Schottky-Kontakt}

Das System Fe-GaAs hat insbesondere seit dem ersten Bericht über das epitaktische Wachstum eines Fe-Films auf einem (001) orientierten n-GaAs Substrat viel Aufmerksamkeit auf sich gezogen [105]. Das epitaktische Wachstum von Fe-Filmen konnte seitdem auf verschiedenen niedrig indizierten GaAs Oberflächen demonstriert werden [42, 105, 115]. Durch die Möglichkeit, Spintronik Bauelemente auf der Basis von Ferromagnet-Halbleiter Systemen zu entwickeln, hat sich das Interesse an diesem System seit den 90er Jahren noch verstärkt [34]. $\mathrm{Fe}-\mathrm{GaAs}$ ist derzeit eines der am besten und intensivsten untersuchten Metall-Halbleiter Systeme [43].

Zu Beginn des Kapitels werden zunächst die bisherigen Erkenntnisse über das epitaktische Wachstum von dünnen Fe-Filmen auf dem in dieser Arbeit untersuchten GaAs\{110\} Substrat zusammengefasst. Von besonderem Interesse sind hierbei Untersuchungen der strukturellen und magnetischen Eigenschaften, die in einem direkten Zusammenhang mit den im weitern Verlauf des Kapitels geschilderten eigenen Untersuchungen stehen. Die Untersuchungen umfassen die Charakterisierung der Filmmorphologie mit dem STM, die strukturelle Analyse mit LEED sowie die Messung des MOKE.

\subsection{Epitaktisches Wachstum - Stand der Wissenschaft}

Das epitaktische Wachstum von Fe auf einem GaAs\{110\} Substrat konnte erstmals 1981 demonstriert werden [110]. Der Fe-Film wächst in diesem Fall in der bcc-Phase auf. Zwischen dem GaAs Substrat und dem Fe-Film existiert die epitaktische Beziehung Fe(110)[001]//GaAs(110)[001]. Abbildung 31 zeigt sowohl die Einheitszelle des GaAs Kristalls als auch die des bcc Fe-Films, die jeweils entlang der (11̄0) Fläche aufgeschnitten wurden. 

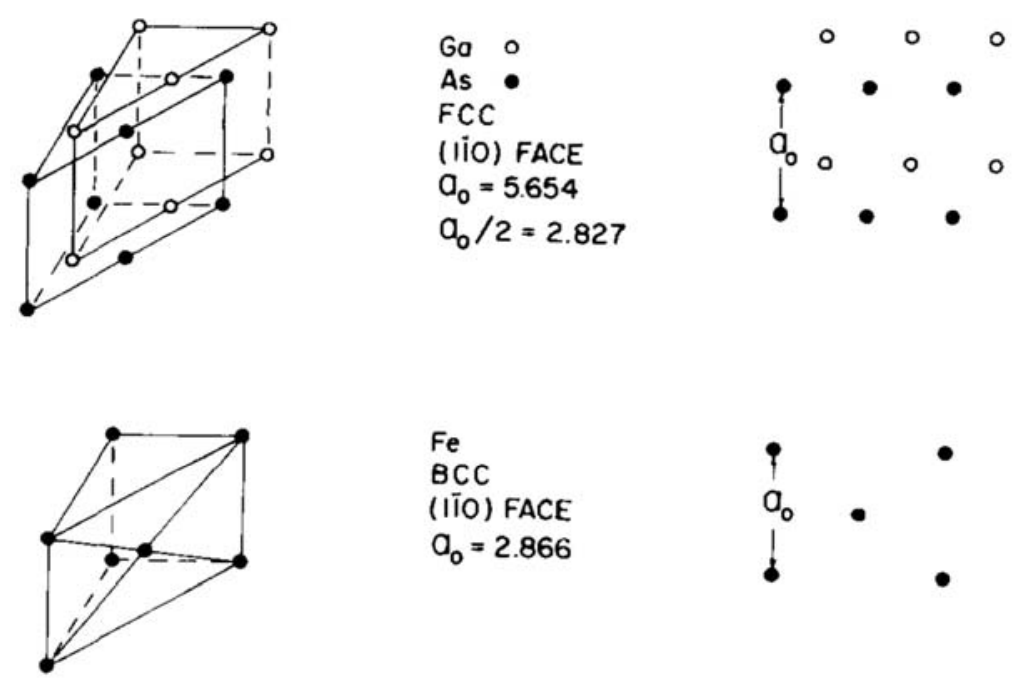

$\mathrm{Fe}$

BCC

(IIT) FACE

$a_{0}=2.866$

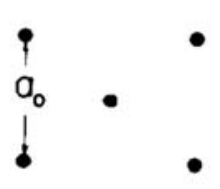

Abbildung 31: Schnitte durch die Einheitszellen des GaAs Substrats und des bcc Fe-Films. Dargestellt sind jeweils die (110) Flächen (aus [110]).

Die Gitterkonstante $\mathrm{a}_{0}$ des GaAs Substrats ist etwa doppelt so groß wie die des bcc Fe-Films. Aufgrund dieser geringen Gitterfehlanpassung der beiden Materialien von nur 1,35 \% sind die Voraussetzungen für das epitaktische Wachstum sehr gut. Das epitaktische Wachstum konnte entsprechend in einem weiten Bereich von Substrattemperaturen zwischen $5^{\circ} \mathrm{C}$ und $225^{\circ} \mathrm{C}$ nachgewiesen werden [110, 116, 124]. Die strukturell besten Filme wurden bei Substrattemperaturen zwischen $175^{\circ} \mathrm{C}$ und $225^{\circ} \mathrm{C}$ gewachsen [110]. Das in der Literatur beschriebene Wachstum von Fe-Filmen auf einer GaAs $\{110\}$ Oberfläche kann in mehrere charakteristische Abschnitte unterteilt werden:

- Zu Beginn des Wachstums bilden sich dreidimensionale Fe-Inseln auf der Substratoberfläche [110, 117]. Das Beugungsmuster bei LEED Untersuchungen zeigt die Bragg-Peaks des Substrats, deren Intensität mit steigender nomineller Schichtdicke des Fe-Films abnimmt [118]. Die Photoelektronen-Spektroskopie (PES) macht deutlich, dass sich im Fe-Film sowohl Ga- als auch As-Atome in signifikanten Mengen befinden und es generell zu einem Aufbrechen der Substratstruktur kommt $[44,116]$. Im Bereich der Grenzfläche wird die Bildung von $\mathrm{Fe}_{2} \mathrm{As}-, \mathrm{Fe}_{3} \mathrm{Ga}-$ und $\mathrm{Fe}_{3} \mathrm{Ga}_{2-\mathrm{x}} \mathrm{As}_{\mathrm{x}^{-}}$ Phasen beobachtet $[44,119,120]$, wobei erhöhte Wachstumstemperaturen die Bildung der Phasen begünstigen [45, 46].

- Zwischen etwa 1 ML und 4 ML nomineller Schichtdicke werden bei LEED Untersuchungen weder die Bragg-Peaks des Substrats noch die des Fe-Films beobachtet [118, 121]. Es konnte gezeigt werden, dass die Bildung der verschiedenen Phasen an der Grenzfläche dazu führt, dass kein Beugungsmuster des Fe-Films beobachtet wird [46]. Aus PES Messungen geht hervor, dass der Anteil von Ga-Atomen im Fe-Film bei steigender Schichtdicke deutlich schneller als der der As-Atome abnimmt. 
- Ab etwa 4 ML Schichtdicke ist der Fe-Film geschlossen und bei LEED Messungen wird das Beugungsmuster des epitaktischen Fe-Films beobachtet [118, 121]. Aus MOKE Untersuchungen geht hervor, dass der Film ab dieser Schichtdicke bei RT ferromagnetisch geordnet ist [118]. Die magnetische Anisotropie besitzt ein uniaxialen Charakter, wobei die leichte Richtung (engl. easy axis, EA) in der Filmebene (engl. in-plane magnetic anisotropy, IPMA) entlang der $<110>$-Richtung des Substrats ausgerichtet ist.

- In Abhängigkeit von der Wachstumstemperatur findet zwischen 24 ML und 50 ML eine Reorientierung der IPMA statt. Nach dieser Reorientierung befindet sich die leichte Richtung in der $<001>$-Richtung [118].

Die geschilderten experimentellen Ergebnisse zeigen, dass es bei dem konventionellen epitaktischen Wachstum von Fe-Filmen auf GaAs\{110\} Substraten generell zur Ausbildung von Schottky-Kontakten mit einem inhomogenen, durchmischten Grenzflächenbereich kommt, die sich darüber hinaus durch nahezu ohmsche I(U)-Kennlinien auszeichnen [122, 123].

Um die Eignung von Fe-GaAs\{110\} Schottky-Kontakten zur Spin-Injektion und -Detektion in möglichen Spintronik Bauelementen zu verbessern, wurde in den vergangen Jahren ein hoher experimenteller Aufwand betrieben, wobei das Hauptaugenmerk auf der Unterdrückung von Reaktionen an der Grenzfläche gelegen hat. Eine deutliche Verbesserung konnte insbesondere durch die Verringerung der Substrattemperatur auf $5^{\circ} \mathrm{C}$ erreicht werden [124]. Dieser experimentelle Befund wird durch DFT Rechnungen der Nukleation von Fe-Atomen auf unterschiedlich terminierten GaAs(001) Substraten unterstützt. Die Berechnungen zeigen, dass es im Regime der Submonolagenbedeckung bis hin zu einer Schichtdicke von 2 ML aufgrund der starken Fe-As-Bindungen generell zu einer Durchmischung der Grenzfläche kommt, sofern eine ausreichend hohe Temperatur gegeben ist [48]. Ab einer Filmdicke von 2 ML stellt hingegen eine abrupte Grenzfläche die energetisch günstigste Konfiguration dar [39, 48]. Die weitere Reduktion der Substrattemperatur, insbesondere während der frühen Phase des Wachstums bis zu einer Schichtdicke von 2 ML, ist demnach von zentraler Bedeutung, um Interdiffusionsprozesse an der Grenzfläche noch effizienter unterdrücken zu können. Unterhalb von $5^{\circ} \mathrm{C}$ wurde das Wachstum von Fe-Filmen auf einer GaAs\{110\} Oberfläche bislang noch nicht untersucht.

\subsection{Fe-GaAs(110) Schottky-Kontakte durch Tieftemperatur- Deposition und anschließendes Annealen: Neue struktu- relle und magnetische Eigenschaften}

Um sowohl eine Durchmischung an der Fe-GaAs(110) Grenzfläche zu vermeiden als auch die strukturellen Eigenschaften des epitaktischen Fe-Films zu optimieren, wurden dünne Fe-Filme mit Dicken von bis zu 10 ML wie in Kapitel 4.3 beschrieben auf der GaAs(110) Spaltfläche präpariert, d.h. im 
UHV bei 130(5) K deponiert und anschließend innerhalb von 2 Stunden bis auf RT annealt. ${ }^{15}$ Sowohl die strukturellen als auch die magnetischen Eigenschaften dieser Filme wurden anschließend im UHV nacheinander mit verschiedenen oberflächensensitiven Messmethoden charakterisiert. Neben dem STM wurden die Filme mit LEED und MOKE untersucht. Um für die LEED und MOKE Messungen Filme mit einer ausreichend großen Oberfläche wachsen zu können, wurden n-GaAs Substrate $\left(2 \cdot 10^{18} 1 / \mathrm{cm}^{3}\right.$ Si dotiert) mit einer Dicke von $380 \mu \mathrm{m}$ verwendet. In den folgenden Kapiteln wird gezeigt, dass mit den verwendeten Messmethoden eine Reihe von Eigenschaften beobachtet werden konnten, die bei konventionell gewachsenen Fe-Filmen nicht auftreten. Insbesondere lassen verschiedene experimentelle Ergebnisse übereinstimmend darauf schließen, dass es durch den verwendeten Wachstumsprozess gelungen ist, einen idealen Fe-GaAs(110) Schottky-Kontakt zu präparieren.

Ein Großteil der hier präsentierten Ergebnisse wurde unter dem Titel „Fe films grown on GaAs(110) in a two-step process: Improved structural and magnetic properties“ in Applied Physics Letters veröffentlicht [54].

\subsubsection{STM}

Um detaillierte Informationen über die Oberflächenmorphologien der Fe-Filme zu gewinnen, wurden STM Messungen an einer Vielzahl von Proben mit unterschiedlichen Schichtdicken durchgeführt. Aus den STM Topografien geht hervor, dass die Fe-Filme im Gegensatz zu konventionell gewachsenen Filmen bereits ab einer Schichtdicke von 2 ML geschlossen sind. Makroskopische Defekte im Fe-Film, die bei niedrigen Substrattemperaturen im Rahmen des konventionellen Wachstums vermehrt auftreten, wurden trotz der LT-Deposition bei keiner der untersuchten Proben beobachtet [42]. In Kapitel 3.1 wurde erläutert, dass es durch den Aufbau des verwendeten XSTM möglich ist, kontrolliert unterschiedliche Bereiche der Probenoberfläche mit der STM Spitze anzusteuern. Dies kann in Verbindung mit Proben, bei denen ein Fe-Film mit leicht ansteigender Filmdicke präpariert worden ist, dazu verwendet werden, um Veränderungen der Oberflächenmorphologie in Abhängigkeit von der Schichtdicke des Fe-Films mit dem STM zu untersuchen. Dieser Ansatz besitzt den Vorteil, dass unterschiedliche Filmdicken unter identischen Bedingungen präpariert werden, so dass der Zusammenhang zwischen der Filmdicke und der Oberflächenstruktur optimal untersucht werden kann. Da die laterale Position der Spitze über ein optisches Mikroskop mit einer Auflösung im Mikrometerbereich bestimmt werden kann, ist eine präzise Zuordnung der Schichtdicke gewährleistet.

\footnotetext{
${ }^{15}$ Die Schichtdicken werden in Fe(110) ML angegeben (2 Å).
} 

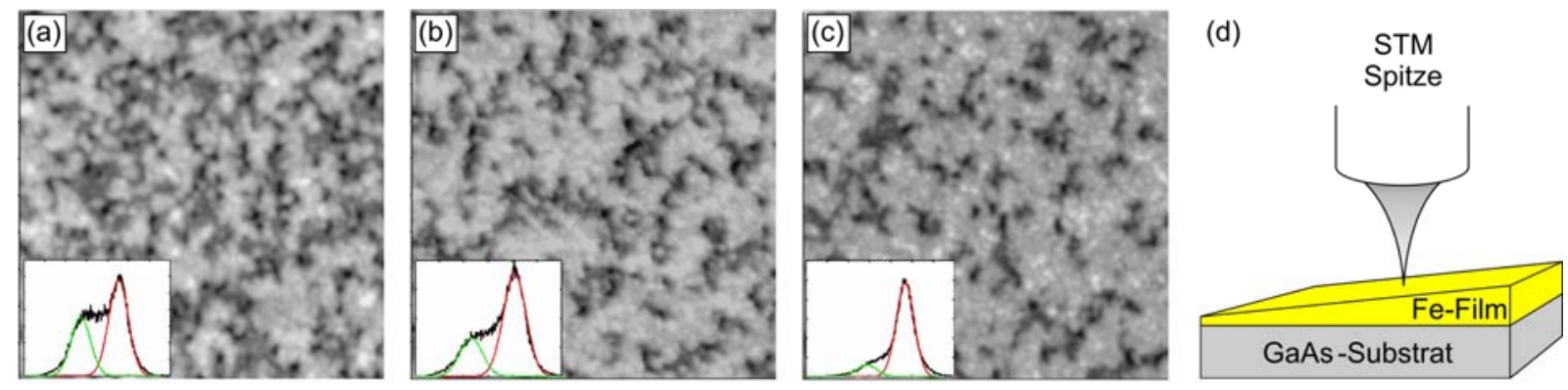

Abbildung 32: (a) - (c) STM Topografien ((100 x 100) nm $\left.\mathrm{nm}^{2}, U_{\text {Bias }}=1,1 \mathrm{~V}, I_{T}=50 \mathrm{pA}\right)$ eines keilförmig aufgewachsenen Fe-Films. Die Topografien wurden in Bereichen mit nominellen Schichtdicken von (a) 6,6 ML, (b) 6,7 ML und (c) 6,9 ML aufgenommen. Die Dynamik der Grauskala beträgt 300 pm. Die Insets zeigen die jeweiligen Histogramme. (d) Skizze der Messanordnung des STM Experiments.

Abbildung 32 (a) - (c) zeigt STM Topografien aus unterschiedlichen Bereichen eines keilförmigen FeFilms mit nominellen Schichtdicken von 6,6 ML, 6,7 ML und 6,9 ML. Der Fe-Film ist in den untersuchten Bereichen atomar flach und die Histogramme der Topografien zeigen lediglich zwei Maxima im Abstand einer Fe(110) Monolage. Es wird deutlich, dass mit zunehmender Schichtdicke zunächst die oberste Fe-Lage aufgefüllt wird. Dies ist charakteristisch für ein 2D-Lagenwachstum, dass bei konventionell gewachsenen Fe-Filmen bislang nicht beobachtet werden konnte.

Neben der Oberflächenmorphologie des jeweiligen Fe-Films kann durch die STM Topografien auch die Qualität der durch den Spaltvorgang erzeugten Bruchfläche charakterisiert werden. Obwohl Substrate mit einer Dicke von $380 \mu$ m verwendet werden mussten, d.h. erheblich dicker als das Optimum zur Erzeugung von defektfreien Spaltflächen von $100 \mu$ m [87], wurden in den STM Topografien nur vereinzelt monoatomare Substratstufen beobachtet. Ein signifikanter Einfluss dieser Defekte auf das Wachstum der Fe-Filme kann daher ausgeschlossen werden.

\subsubsection{LEED}

Die schichtdickenabhängige Evolution des LEED Beugungsmusters bei dem konventionellen Wachstum von Fe-Filmen auf einer GaAs\{110\} Oberfläche wurde in Kapitel 5.1 dargestellt. Charakteristisch ist das Fehlen eines Beugungsmusters bei Schichtdicken zwischen etwa 1 ML und 4 ML, was auf die Ausbildung verschiedener As- und Ga-haltiger Phasen im Bereich der Grenzfläche zurückgeführt werden kann [46]. Im Gegensatz dazu zeigt Abbildung 33 die schichtdickenabhängigen Veränderungen der LEED Beugungsmuster von Fe-GaAs(1110) Schottky-Kontakten, die in dem zweistufigen Präparationsverfahren hergestellt wurden. 

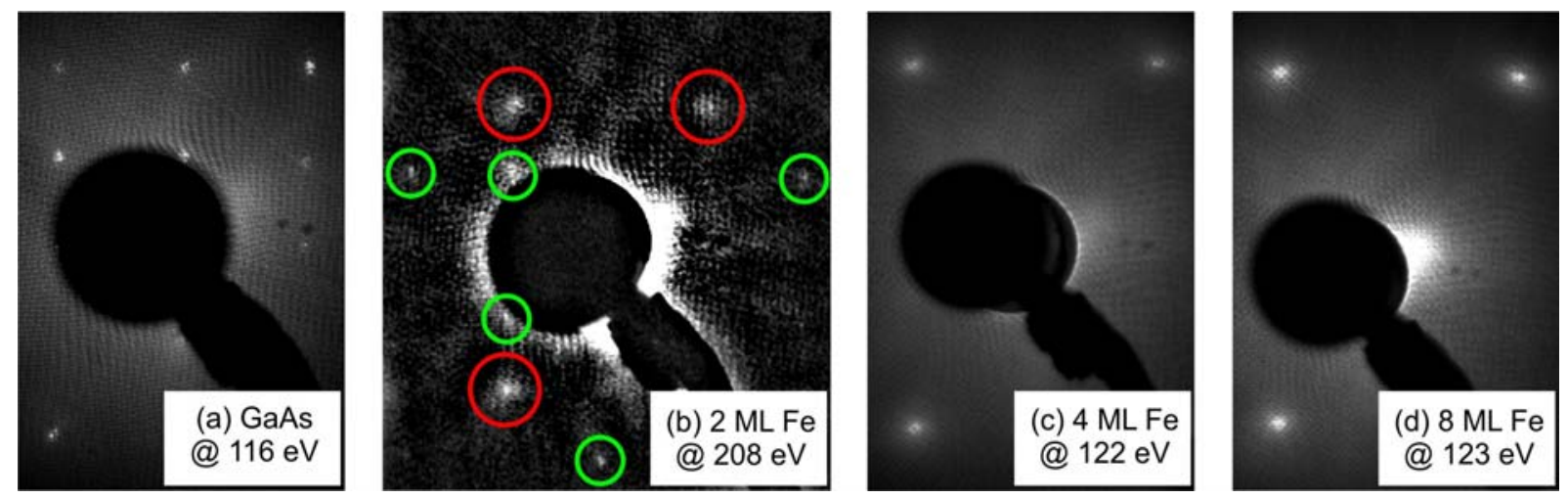

Abbildung 33: LEED Beugungsbilder (a) der freien GaAs(1110) Spaltfläche und (b) - (d) nach dem Wachstum von Fe-Filmen mit verschiedenen nominellen Schichtdicken. Bei einer Schichtdicke von $2 M L$ ist sowohl das Beugungsmuster des GaAs Substrats (grüne Kreise) als auch das des Fe-Films (rote Kreise) zu erkennen. Die Verbreiterung des Fe-Beugungsmusters ist bei einer Schichtdicke von etwa 8 ML am geringsten.

Neben dem Beugungsbild der freien GaAs(110) Spaltfläche sind die Beugungsbilder von Fe-Filmen mit unterschiedlichen nominellen Schichtdicken dargestellt. ${ }^{16}$ Sie lassen sowohl Aussagen über die Metall-Halbleiter Grenzfläche als auch über die strukturellen Eigenschaften der Fe-Filme zu:

- Es ist trotz der LT-Deposition zur Ausbildung epitaktischer bcc Fe-Filme gekommen. Die epitaktische Beziehung ist identisch mit der von Fe-Filmen, die bei RT oder höheren Substrattemperaturen gewachsen wurden.

- Im Gegensatz zu konventionell gewachsenen Fe-Filmen ist bei einer Schichtdicke von 2 ML sowohl das Beugungsmuster des GaAs Substrats als auch bereits das des Fe-Films zu erkennen. Entsprechend existiert kein Schichtdickenbereich, in dem kein Beugungsmuster beobachtet werden kann. Eine signifikante Bildung von $\mathrm{Fe}_{2} \mathrm{As}-, \mathrm{Fe}_{3} \mathrm{Ga}$ - oder $\mathrm{Fe}_{3} \mathrm{Ga}_{2-\mathrm{x}} \mathrm{As}_{\mathrm{x}}-\mathrm{Phasen}$ im Bereich der Grenzfläche kann daher ausgeschlossen werden. Das Beugungsbild deutet an, dass die Struktur des Substrats bei dem Wachstumsprozess nicht aufgebrochen wurde.

- Verglichen mit der apparativen Auflösung, die anhand der Verbreiterung von Beugungspunkten der nahezu perfekten GaAs(110) Spaltfläche bestimmt werden kann, beträgt die Verbreiterung des Fe-Beugungsbilds bei einer Schichtdicke von 8 ML nur 30 \%. Diese geringfügige Verbreiterung zeigt wie auch die STM Topografien, dass der Fe-Film in diesem Schichtdickenbereich im Gegensatz zu konventionell gewachsenen Filmen einen ausgeprägten 2D-Charakter besitzt.

${ }^{16}$ Die in diesem Kapitel präsentierten Messdaten wurden an dem LEED-Auger-System ErLEED3000D der Firma Spex gewonnen. 


\subsubsection{MOKE}

Neben den strukturellen Eigenschaften wurden auch die magnetischen Eigenschaften der Fe-Filme anhand von MOKE Messungen charakterisiert. Um diese Messungen in-situ durchführen zu können, d.h. ohne den Einfluss einer für ex-situ Messungen notwendigen Passivierungsschicht, wurde im Rahmen dieser Arbeit / Masterarbeit D. Lyzwa eine UHV Kammer entwickelt, die direkt mit der zur Präparation der Fe-Filme verwendeten UHV Präparationskammer verbunden werden kann [125]. Der Aufbau der Kammer erlaubt es, den longitudinalen Kerr-Effekt bei RT zu messen, d.h. die Magnetisierung in der Filmebene parallel zur Ebene des einfallenden Lichtes. Die Probe kann relativ zu dem angelegten externen Feld gedreht werden, so dass Hysteresekurven in den in der Filmebene liegenden Hauptanisotropie-Richtungen [110] und [001] von konventionell gewachsenen Fe-GaAs(110) Filmen aufgenommen werden können [118, 126].
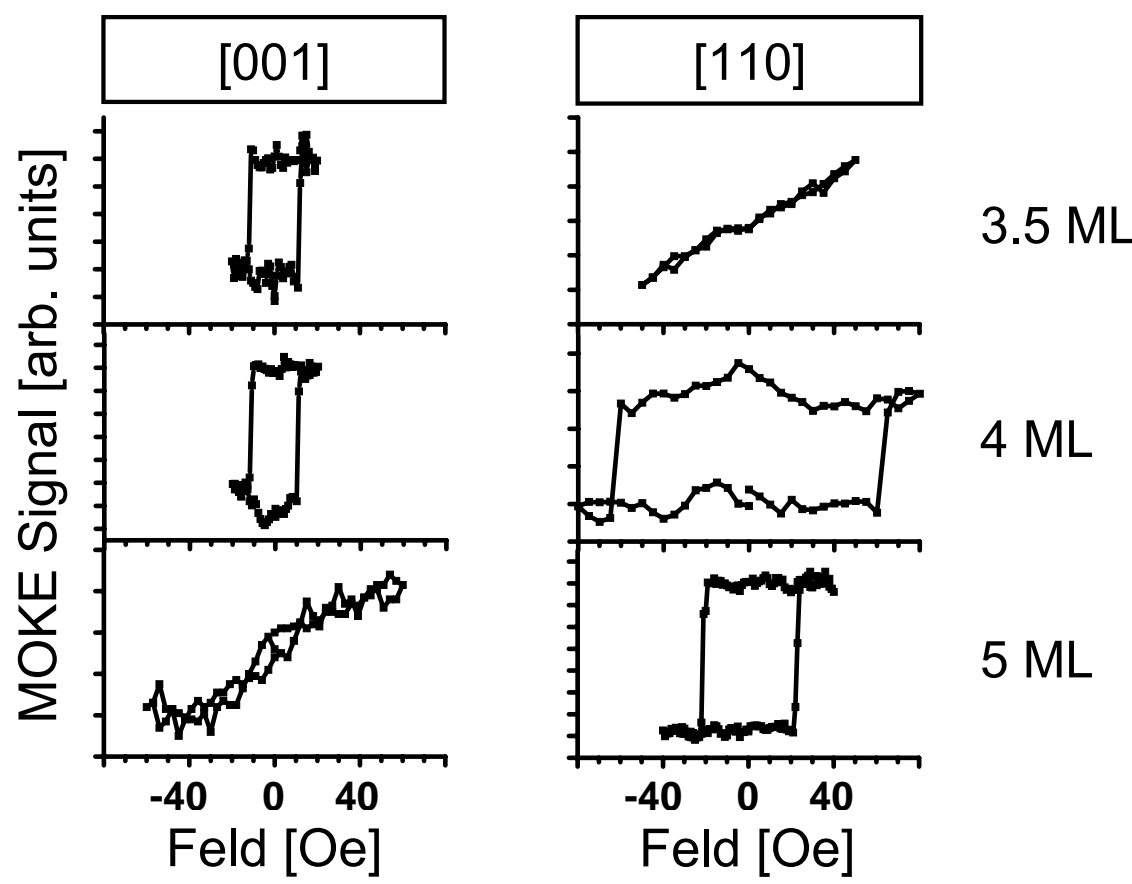

Abbildung 34: Hysteresekurven der in-plane Magnetisierung von Fe-Filmen mit 3,5 ML, 4 ML und 5 ML Dicke. Das externe Feld wurde in der [001]- und [110]-Richtung der Probe angelegt.

Hysteresekurven von Fe-Filmen unterschiedlicher Dicken zeigt Abbildung 34. Im Gegensatz zu FeFilmen, die konventionell bei RT auf einer GaAs $\{110\}$ Oberfläche gewachsen wurden, sind die Filme 
bereits ab einer Schichtdicke von 3,5 ML bei RT ferromagnetisch geordnet [118]. ${ }^{17}$ Ein sprunghaftes Umklappen der Magnetisierung findet statt, wenn das externe Feld in der [001]-Richtung der Probe angelegt wird. In der [110]-Richtung wird hingegen keine Remanenzmagnetisierung beobachtet. Im Rahmen des Stoner-Wohlfahrt-Modells, das das Magnetisierungsverhalten einer homogen magnetisierten Probe mit uniaxialer Anisotropie durch eine kohärente Rotation beschreibt, entspricht die leichte bzw. harte Richtung der [001]- bzw. [110]-Richtung der Probe [127]. Bei einer Schichtdicke von 4 ML werden in beiden Richtungen rechteckige Hystereseschleifen mit unterschiedlichen Koerzitivfeldern beobachtet. Das Ummagnetisierungsverhalten des 5 ML dicken Films ist schließlich entgegengesetzt zu dem des 3,5 ML dicken Films, d.h. die leichte bzw. harte Richtung der Magnetisierung ist bei dieser Schichtdicke entlang der [110]- bzw. [001]-Richtung der Probe orientiert. Die Hysteresekurven in Abbildung 34 machen deutlich, dass bei etwa 4 ML Filmdicke eine Reorientierung der IPMA stattfindet, die bei konventionell gewachsenen Fe-Filmen nicht beobachtet wurde. Sie zeigen darüber hinaus, dass bei den jeweiligen Koerzitivfeldern eine Erhöhung des angelegten Feldes um nur 1 Oersted zu einem Umklappen der Magnetisierung führt. Eine graduelle Veränderung der Magnetisierung findet nicht statt, die bei RT gewachsenen Fe-Filmen beobachtet wird und auf Defekte bzw. die Bildung von Ga- bzw. As-haltigen Phasen an der Grenzfläche zurückgeführt wird [128].

Mit den drei verwendeten Messmethoden konnten verschiedene magnetische und strukturelle Eigenschaften beobachtet werden, die sich zum Teil stark von denen unterscheiden, die für konventionell gewachsene Fe-Filme auf einer GaAs $\{110\}$ Oberfläche typisch sind. Insbesondere konnten Charakteristika, wie z.B. das Fehlen eines Beugungsmusters bei Schichtdicken zwischen 1 ML und 4 ML oder die graduelle Veränderung der Magnetisierung bei dem Koerzitivfeld, die bei RT gewachsenen Filmen auf das Vorhandensein von verschiedenen Phasen bzw. Defekten im Bereich der durchmischten Grenzfläche zurückgeführt werden, nicht festgestellt werden [118, 121, 128]. Aus den experimentellen Ergebnissen kann übereinstimmend geschlossen werden, dass es durch das verwendete Präparationsverfahren gelungen ist, Reaktionen an der Grenzfläche effektiv zu unterdrücken. Es handelt sich demnach um ideale Fe-GaAs\{110\} Schottky-Kontakte mit einer abrupten Grenzfläche, bei denen darüber hinaus auch die strukturellen Eigenschaften der epitaktischen Fe-Filme verbessert werden konnten.

${ }^{17}$ Bei geringeren Schichtdicken konnte bei RT keine ferromagnetische Ordnung beobachtet werden, obwohl die Filme bereits geschlossen sind. Im Fall von epitaktischen Fe-GaAs(001) Filmen wurde gezeigt, dass die Curie-Temperatur mit geringer werdender Filmdicke abnimmt und sich bei einer Schichtdicke von 3,6 ML bereits im Bereich von RT befindet [144]. Es ist daher wahrscheinlich, dass auch bei den hier untersuchten Filmen eine Curie-Temperatur unterhalb von RT dafür verantwortlich ist, dass bei Schichtdicken unterhalb von 3,5 ML keine Hystereseschleifen beobachtet werden konnten. 


\section{Atomare strukturelle Eigenschaften: XSTM}

In Kapitel 5 wurden verschiedene Messergebnisse präsentiert, die übereinstimmend darauf schließen lassen, dass durch die LT-Deposition eines dünnen Fe-Films auf der GaAs(110) Spaltfläche und das anschließende Annealen bis auf RT ideale Schottky-Kontakte mit einer abrupten, homogenen Metall-Halbleiter Grenzfläche präpariert werden können. Die derzeit einzige Messmethode, um diese strukturellen Eigenschaften lokal auf der atomaren Skala zu verifizieren, ist die Raster-Tunnel-Mikroskopie in Querschnittsgeometrie.

Im Verlauf des Kapitels werden anhand der Morphologie der Fe-GaAs(110) Spaltfläche zunächst die Bereiche identifiziert, die ausreichend gut gespalten sind, um durch die XSTM Messungen Informationen über die atomare Struktur und die elektronischen Eigenschaften des Schottky-Kontakts zu gewinnen. Nachdem sowohl die Tunnelspitze als auch die lokalen Tunelbdingungen in der RLZ charakterisiert wurden, wird detailliert auf die Bestimmung der strukturellen Eigenschaften des untersuchten Schottky-Kontakts eingegangen.

Um ein Fe-GaAs(110) Schottky-Kontakt, wie er bereits mit LEED, STM und MOKE charakterisiert wurde, für die Untersuchung mit dem XSTM zu präparieren, wurde das in den Kapiteln 4.2 und 4.3 beschriebe Verfahren verwendet. Als Substrat wurde ein n-GaAs Wafer mit einer Si-Konzentration von $8 \cdot 10^{17} 1 / \mathrm{cm}^{3}$ gewählt. Bei dieser Dotierung ist gewährleistet, dass zum einen die Verteilung von Dotieratomen in der RLZ ausreichend diskret ist, um den Effekt einzelner Dotieratome auf den Potentialverlauf in der RLZ untersuchen zu können. Zum anderen wird erreicht, dass die Ausdehnung der RLZ kleiner als $50 \mathrm{~nm}$ ist, so dass der gesamte Bereich mit dem XSTM zusammenhängend atomar aufgelöst abgebildet werden kann. Um einen möglichst homogenen Fe-Film zu erhalten, wurde aufgrund der in den LEED und STM Messungen erkennbaren Tendenzen ein Film mit einer Schichtdicke von $7 \mathrm{ML}$ deponiert. Abgesehen von der Dotierkonzentration des Substrats wurde der SchottkyKontakt unter denselben Bedingungen präpariert, bei denen auch die mit LEED, STM und MOKE untersuchten Proben gewachsen wurden. Sowohl der für die Querschnittsgeometrie notwendige zweite Spaltvorgang als auch die XSTM Messungen wurden bei RT durchgeführt.

\subsection{Charakterisierung der Spaltfläche}

STM Untersuchungen in Querschnittsgeometrie beruhen auf der Möglichkeit, durch einen Spaltvorgang eine Oberfläche zu erzeugen, deren atomare Struktur die der Probe vor dem Spaltvorgang widerspiegelt. In Kapitel 4.4 wurde bereits dargelegt, dass diese idealen Voraussetzungen bei komplexen Probensystemen wie Metall-Halbleiter Kontakten nur sehr schwer zu erreichen sind. Es ist daher zu- 
nächst notwendig, anhand der Morphologie der Bruchfläche zu überprüfen, ob bzw. in welchen Bereichen die Spaltfläche die oben genannten Bedingungen erfüllt. Abbildung 35 zeigt dazu eine XSTM Topografie aus dem Bereich der Grenzfläche in einer 3D-Darstellung.

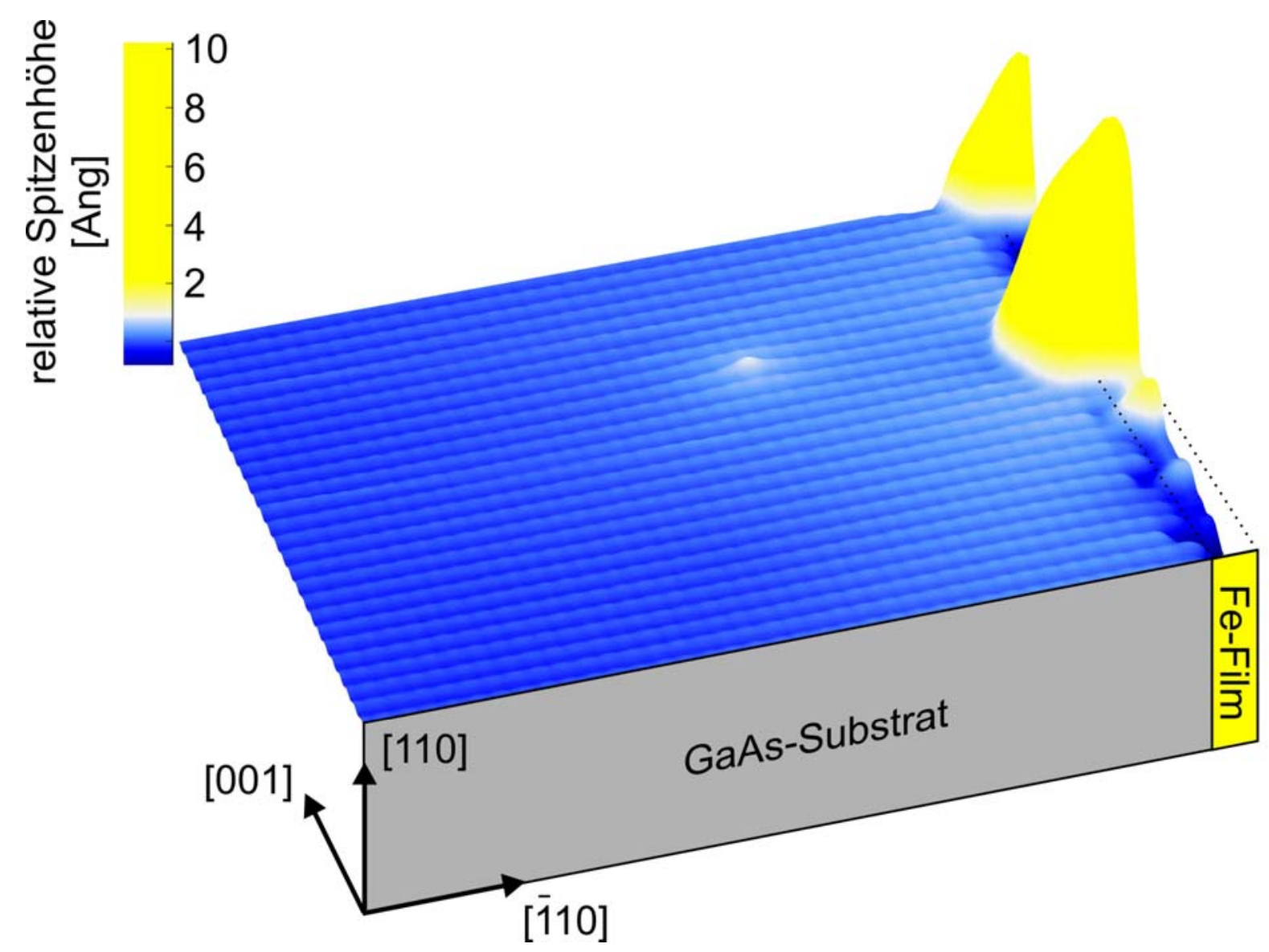

Abbildung 35: XSTM Topografie ((15 x 15) $\left.\mathrm{nm}^{2}, U_{\text {Bias }}=-2,4 \mathrm{~V}, \mathrm{I}_{T}=100 \mathrm{pA}\right)$ der Bruchfläche des $\mathrm{Fe}-\mathrm{GaAs}(\overline{1} 10)$ Schottky-Kontakts, die durch den zweiten Spaltvorgang erzeugt wurde. Der Ausschnitt zeigt den Bereich der Metall-Halbleiter Grenzfläche in einer 3D-Darstellung.

Der Bereich des n-GaAs Substrats kann durch verschiedene charakteristische Eigenschaften der GaAs(110) Spaltfläche, die mit dem STM abgebildet werden können (Kapitel 3.2 und 3.3), identifiziert werden. In STM Topografien ist ein wesentlicher Punkt die deutliche atomare Korrugation der unrekonstruierten Oberflächenlage (im Fall der in Abbildung 35 gezeigten Topografie wird bei der gewählten Bias-Spannung von -2,4 V das As-Untergitter abgebildet). Es ist deutlich zu erkennen, dass es im Bereich des GaAs Substrats gelungen ist, bei dem zweiten Spaltvorgang eine atomar glatte Oberfläche zu präparieren, die sich optimal zur strukturellen Analyse mit dem XSTM eignet. Inhomogenitäten, die in der Topografie in Abbildung 35 zu erkennen sind (leichte Erhöhung in der Mitte des Ausschnitts), können direkt auf atomare Defekte im Kristallgitter des GaAs Substrats zurückgeführt 
werden. Auf die Identifizierung der verschiedenen Defekte, die im Bereich des GaAs Substrats beobachtet werden, wird in Kapitel 6.4 detailliert eingegangen.

In Bezug auf den Fe-Film zeigt die in Abbildung 35 dargestellte Topografie, dass es bei dem bei RT durchgeführten zweiten Spaltvorgang nicht gelungen ist, den 7 ML dicken Fe-Film in der Ebene des GaAs Substrats zu spalten. Die Morphologie lässt darauf schließen, dass der Fe-Film plastisch deformiert wurde, so dass keine Informationen über die atomare Struktur vor dem Spaltvorgang gewonnen werden können. Entlang der Grenzfläche ragt der Fe-Film an einigen Stellen maximal $1 \mathrm{~nm}$ über die Spaltebene des GaAs Substrats hinaus. Die XSTM Topografie vermittelt den Eindruck, dass der Fe-Film an diesen Stellen bis in den Bereich des GaAs Substrats ausgedehnt ist. In Abbildung 35 ist deutlich zu erkennen, dass dieser Effekt umso ausgeprägter ist, je weiter der Fe-Film über die Spaltebene des Substrats hinausragt. Darüber hinaus fällt auf, dass auch die scheinbare Form des Fe-Films an diesen Stellen nahezu identisch ist. Es handelt sich hierbei um ein Artefakt der Abbildung, das wie bereits anhand von Abbildung 29 erläutert durch die Ausdehnung der STM Spitze verursacht wird. Da die STM Spitze einen größeren Krümmungsradius als der über die Spaltebene hinausragende Fe-Film besitzt, wird an diesen Stellen jeweils die STM Spitze abgebildet. Es gibt keinen Hinweis darauf, dass das GaAs Substrat in diesen Bereichen nicht atomar glatt gespalten ist.

Aufgrund der geringen Dicke des Fe-Films sind die Abbildungsartefakte nicht so stark ausgeprägt, wie bei dem im Kapitel 3.4 beschriebenen Au-GaAs(110) Schottky-Kontakt, so dass der Übergangsbereich von dem GaAs Substrat zu dem Fe-Film an vielen Stellen mit dem STM zugänglich ist. In diesen Bereichen ist es erstmals möglich, sowohl die strukturellen als auch die elektronischen Eigenschaften eines Fe-GaAs(110) Schottky-Kontakts von der RLZ bis hin zur Metall-Halbleiter Grenzfläche mit atomarer Auflösung zu untersuchen.

\subsection{Charakterisierung der Tunnelspitze}

In Kapitel 3.3.1 wurde geschildert, dass es bei XSTM Untersuchungen von GaAs\{110\} Spaltflächen generell zu dem Effekt des TIBB kommt. Die Interpretation der Messdaten wird erheblich erschwert, da die Tunnelbedingungen durch das TIBB modifiziert werden und nicht allein über die angelegte Bias-Spannung bestimmt werden kann, welche Zustände zu dem Tunnelstrom beitragen. Wie stark das TIBB ausgeprägt ist, hängt dabei sehr stark von dem Krümmungsradius der STM Spitze ab [97]. Da diese Größe bei einer STM Messung im Allgemeinen nicht zugänglich ist, können beispielsweise die in Kapitel 2.6 beschrieben REM Untersuchungen von identisch präparierten Spitzen einen ersten Anhaltspunkt liefern [79]. Eine quantitative Bestimmung ist auf diesem Weg allerdings nicht möglich, da STM Spitzen trotz eines identischen Präparationsprozesses sehr unterschiedliche Geometrien aufweisen können, die sich darüber hinaus auch im Laufe der Untersuchung einer Probe stark verändern kön- 
nen. Eine belastbare Bestimmung der 3D-Geometrie der Spitze kann daher nur durch eine Charakterisierung der Spitze während der Messung erfolgen.

Im vorangegangen Kapitel wurde beschrieben, dass es im Bereich des Metallfilms an einigen Stellen zu einem Abbildungsartefakt kommt, bei dem jeweils die STM Spitze abgebildet wird. Durch dieses Artefakt wird es ermöglicht, den tatsächlich während der Messung vorhandenen Krümmungsradius der Spitze zu bestimmen.

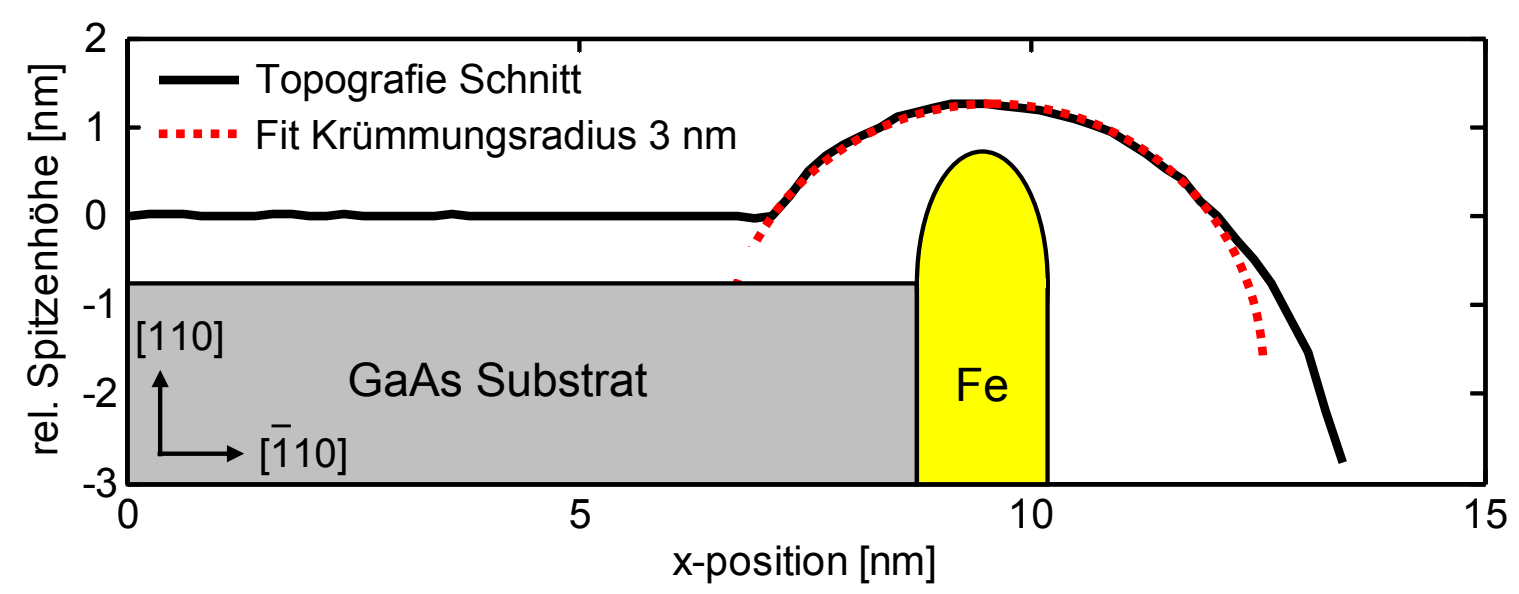

Abbildung 36: Bestimmung des Krümmungsradius der STM Spitze durch eine XSTM Topografie aus dem Bereich der Metall-Halbleiter Grenzfläche. Unter dem senkrecht auf die Grenzfläche zulaufenden Schnitt durch die Topografie befindet sich eine schematische Darstellung der Struktur der Probe. Die Position der Grenzfläche stimmt mit der experimentell bestimmten Lage überein.

Abbildung 36 zeigt dazu einen Schnitt durch eine XSTM Topografie des Fe-GaAs(110) Grenzflächenbereichs. Schnitte aus anderen Bereichen der Topografie, in denen es ebenfalls zu dem Abbildungsartefakt kommt, zeigen im Bereich des Metallfilms einen nahezu identischen Verlauf. Der Fit an die Messdaten zeigt, dass der Krümmungsradius der STM Spitze in diesem Fall lediglich rund 3 nm beträgt. Vergleichbare Analysen der Spitzengeometrie im Bereich des Tunnelkontakts können bei vielen XSTM Topografien des Fe-GaAs(110) Schottky-Kontakts durchgeführt werden. Es zeigt sich, dass der Krümmungsradius der Spitze von anfänglich rund 2,5 nm im Laufe von etwa 12 Stunden Messbetrieb nur geringfügig auf zirka $3 \mathrm{~nm}$ zunimmt.

\subsection{Charakterisierung der lokalen Tunnelbedingungen in der Raumladungszone}

Das TIBB bei STM Untersuchungen von GaAs $\{110\}$ Spaltflächen hängt neben dem Krümmungsradius der STM Spitze auch von ihrer Austrittsarbeit $\phi_{T}$ ab, die in der Regel nur näherungsweise durch die 
Messung der lokalen effektiven Barrierenhöhe bestimmt werden kann (siehe Kapitel 3.3.1). Dies kann zu erheblichen Problemen bei der Interpretation von Messdaten führen, da von $\phi_{T}$ unter anderem abhängt, ob es bei einer gegebenen Bias-Spannung zu einer positiven oder negativen Bandverbiegung kommt. Dies hat zur Folge, dass anhand der Bias-Spannung nicht mit Bestimmtheit festgestellt werden kann, welche Tunnelkanäle in welchem Umfang zu dem Tunnelstrom beitragen. Es ist daher üblich, derartige Informationen durch charakteristische Merkmale wie die in Kapitel 3.3 beschriebene Spannungsabhängigkeit der atomaren Korrugation aus den Messdaten heraus zu rekonstruieren [130]. Bei den XSTM Messungen im Bereich der RLZ des Fe-GaAs(110) Schottky-Kontakts ist zu beachten, dass sich die Bedingungen des Tunnelexperiments durch das elektrostatische Potential in der RLZ lokal verändern. Um die XSTM Daten richtig interpretieren und auswerten zu können, ist es daher erforderlich, die Tunnelbedingungen auch in Abhängigkeit von dem Abstand zwischen STM Spitze und Metall-Halbleiter Grenzfläche zu charakterisieren.

Am Ende des Kapitels wird gezeigt, dass die Ausdehnung $w_{\text {STM }}$ des Bereichs, in dem veränderte Tunnelbedingungen beobachtet werden, darüber hinaus erste Anhaltspunkte über die Weite der RLZ und die damit gemäß (1-5) zusammenhängende SBH des Systems beinhaltet.

Um zunächst die lokale Variation der Tunnelbedingungen bei positiven Probenspannungen charakterisieren zu können, zeigt Abbildung 37 (a) im oberen Teil eine XSTM Topografie des Bereichs der RLZ. Am rechten Rand der Topografie ist der Fe-Film zu erkennen. Die relative Spitzenhöhe ist farbkodiert dargestellt. Die Originaldaten wurden um die Verkippung der Probe relativ zum Koordinatensystem des STM Scanners korrigiert. Derart modifizierte Topografien werden im Folgenden als normierte Topografien bezeichnet. Die Verkippung resultiert sowohl aus dem Einbau der Probe in den Probenhalter als auch aus dem Einbau in den STM Scanner und wurde anhand von STM Topografien ermittelt, die in einigen $\mu \mathrm{m}$ Entfernung von dem Metall-Halbleiter Übergang aufgenommen wurden, so dass ein Einfluss des Schottky-Kontakts ausgeschlossen werden kann. 


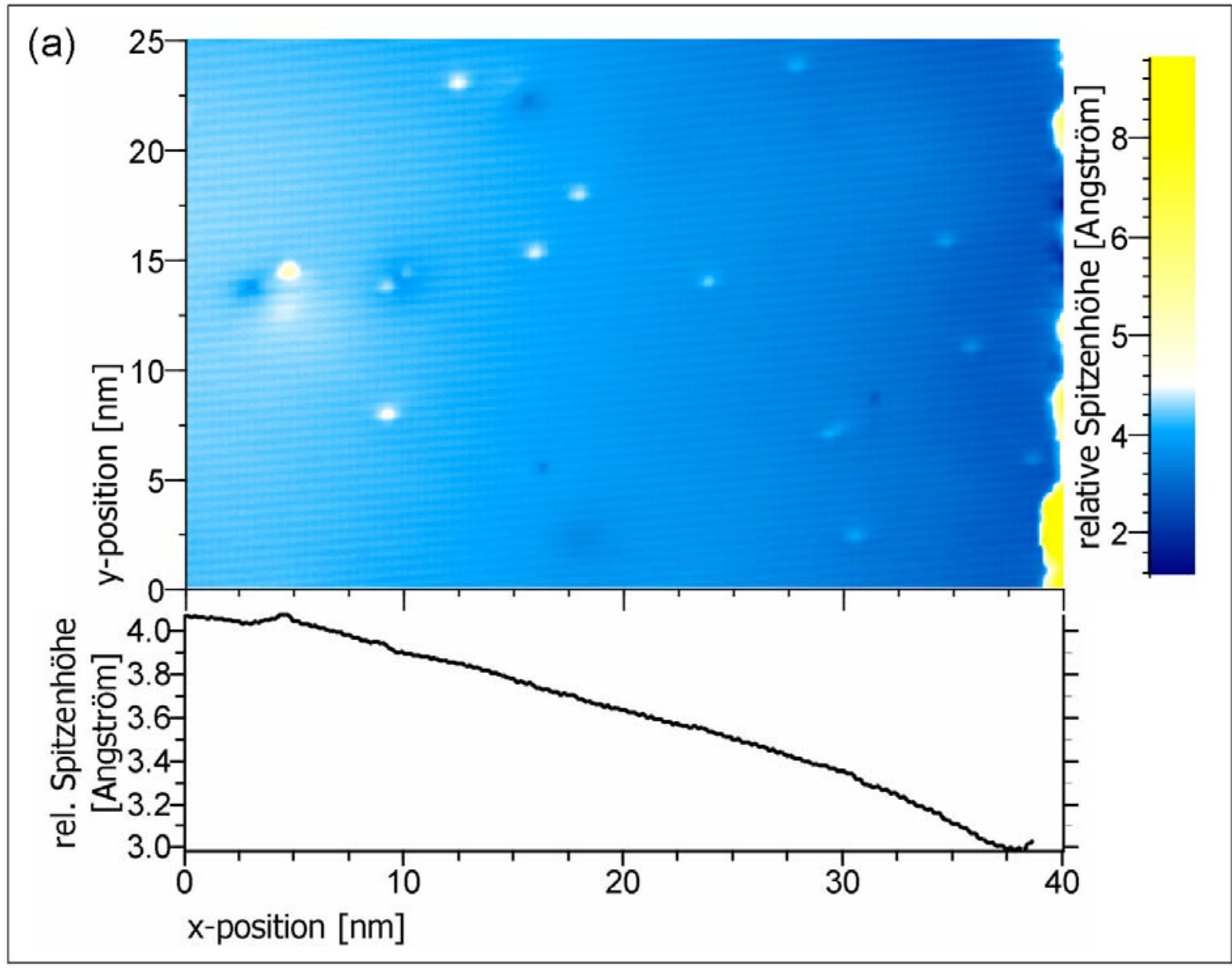

(b)

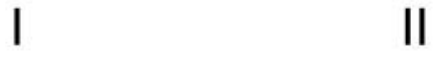

III
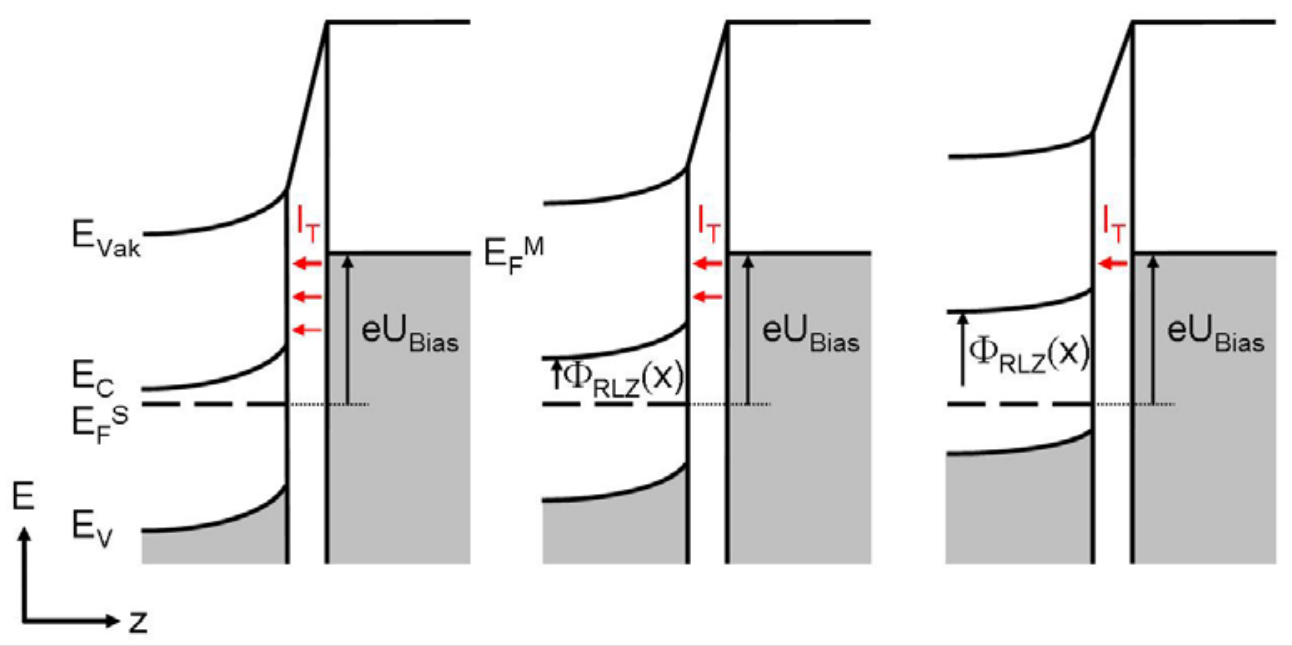

Abbildung 37: (a) XSTM Topografie bei positiver Bias-Spannung ( $(40 \times 25) \mathrm{nm}^{2}, U_{\text {Bias }}=1,8 \mathrm{~V}$, $I_{T}=100 \mathrm{pA}$ ). Sowohl die RLZ als auch der angrenzende Metallfilm sind zu erkennen. Der untere Teil zeigt ein in x-Richtung verlaufenden Schnitt durch die Topografie im Bereich des GaAs Substrats, bei dem über alle Messpunkte in y-Richtung gemittelt wurde. (b) Schematische 1D-Bandmodelle des Tunnelexperiments in der RLZ bei geringer werdenden Abständen (I - III) zum Metallfilm. 
In der normierten Topografie in Abbildung 37 (a) verursacht die atomar glatt gespaltene GaAs(110) Oberfläche keinen strukturellen Kontrast. Bei der Verringerung der relativen Spitzenhöhe zur Grenzfläche hin handelt sich daher ausschließlich um einen elektronischen Kontrast. ${ }^{18}$ Der unter der Topografie gezeigte Schnitt macht deutlich, dass die relative Spitzenhöhe im Bereich der RLZ durchschnittlich um mehr als 1 Ångström abnimmt (der leichte Anstieg einige nm vor dem Fe-Film wird in Kapitel 7.1 ausführlich diskutiert). Dieser starke elektronische Kontrast kann wie folgt durch den Einfluss des elektrostatischen Potentials auf die Tunnelbedingungen in der RLZ erklärt werden kann:

- Anhand der IVCHAR Simulationen in Kapitel 3.3.2 wurde gezeigt, dass außerhalb der RLZ ausschließlich leere Leitungsbandzustände zu dem Tunnelstrom beitragen (Bandschema I in Abbildung 37 (b)).

- In der RLZ werden die Bänder im Halbleiter durch das lokale elektrostatische Potential $V_{R L Z}(x)$ relativ zur Fermi-Energie um den Betrag $\phi_{R L Z}(x)=-e V_{R L Z}(x)$ affin verschoben. $\phi_{R L Z}(x)$ nimmt mit geringer werdendem Abstand zum Metallfilm zu, so dass immer weniger unbesetzte Zustände der Probe zu dem Tunnelstrom beitragen können (Bandschema II und III in Abbildung 37 (b)). Um den Setzstrom der Konstantstromtopografie zu erreichen, muss die Spitze durch den Regelkreis weiter an die Probenoberfläche angenähert werden, so dass die relative Spitzenhöhe bei positiver Bias-Spannung umso geringer wird, je dichter sich die Spitze an dem Fe-Film befindet.

Durch den geringen Krümmungsradius der Spitze von nur $3 \mathrm{~nm}$ kann davon ausgegangen werden, dass die Oberflächenbandverbiegung $\phi_{S B B}\left(x_{\text {Spize }}\right)$ direkt unter dem Apex der Spitze, die sich aus $\phi_{\text {TIBB }}$ und $\phi_{R L Z}\left(x_{\text {Spizze }}\right)$ zusammensetzt, auch in der Nähe der Metall-Halbleiter Grenzfläche weniger als 1,4 eV beträgt. In Kapitel 7.1.2 wird dies durch 3D-Simulationen des TIBB verifiziert. Sie ist damit stets geringer als die fundamentale Bandlücke von GaAs bei RT, so dass bei der verwendeten Setzspannung von 1,8 V im gesamten Bereich der RLZ ausschließlich die unbesetzten, an den Ga-Atomen lokalisierten $\mathrm{C}_{3}$ - und $\mathrm{C}_{4}$-Zustände zum Tunnelstrom beitragen.

\footnotetext{
${ }^{18}$ Auf die lokalisierten Erhebungen und Vertiefungen, die durch atomare Defekte verursacht werden, wird im Kapitel 6.4 detailliert eingegangen.
} 


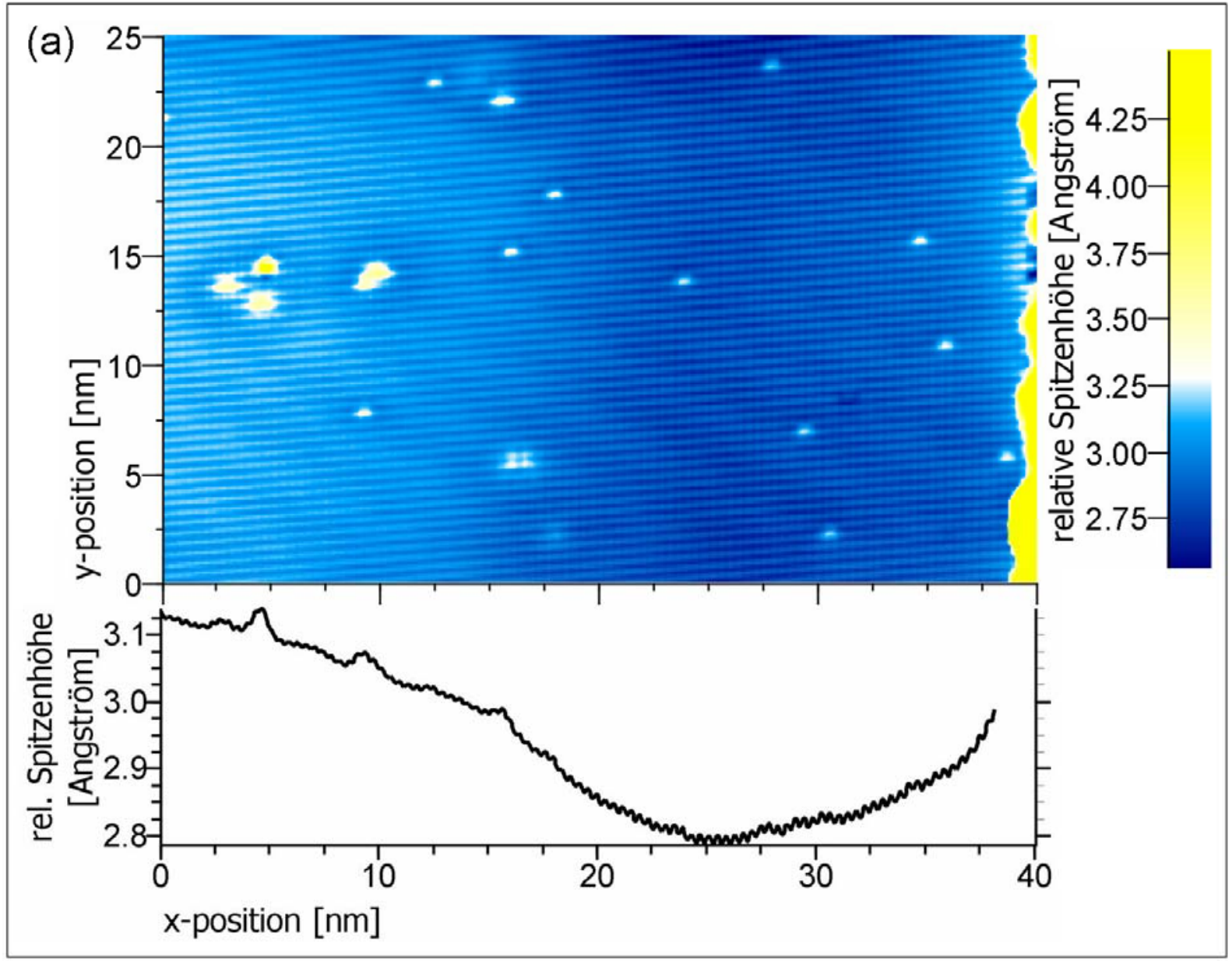

(b)

II

III
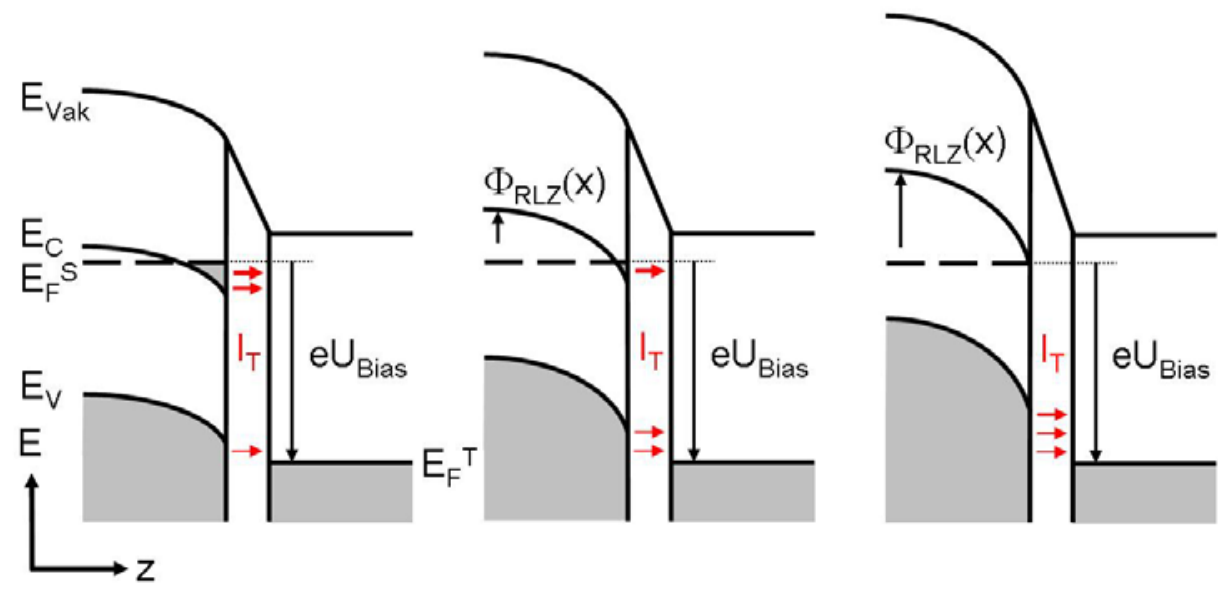

Abbildung 38: (a) XSTM Topografie bei negativer Bias-Spannung (selber Ausschnitt wie in Abbildung 37, $U_{\text {Bias }}=-2,4 \mathrm{~V}, I_{T}=100 \mathrm{pA}$ ). (b) 1D-Bandmodelle des Tunnelexperiments.

Um auch die lokale Variation der Tunnelbedingungen bei negativen Probenspannungen untersuchen zu können, wurde der in Abbildung 37 (a) gezeigte Bereich der Probenoberfläche zusätzlich bei einer Bias-Spannung von -2,4 V abgebildet. Die entsprechende normierte XSTM Topografie ist in 
Abbildung 38 (a) dargestellt. Auch bei der Abbildung mit negativer Bias-Spannung ist in der Topografie ein ausgeprägter elektronischer Kontrast zu erkennen. Der Schnitt durch die Topografie macht deutlich, dass die relative Spitzenhöhe im Durchschnitt wie auch bei positiver Bias-Spannung zunächst mit geringer werdendem Abstand zum Fe-Film abnimmt. In einem Abstand von etwa $15 \mathrm{~nm}$ zum Fe-Film beginnt die Topografie allerdings wieder anzusteigen. Die Höhenvariation ist mit etwa 0,3 Ångström deutlich geringer als bei positiver Bias-Spannung. In den Kapiteln 3.3.1 und 3.3.2 wurde erläutert, dass bei negativer Probenspannung durch das TIBB sowohl besetzte Zustände aus dem Valenzband als auch besetzte Zustände aus der Akkumulationszone an der Oberfläche der GaAs(110) Spaltfläche zu dem Tunnelstrom beitragen können. Der topografische elektronische Kontrast in Abbildung 38 (a) kann dadurch erklärt werden, dass das relative Gewicht dieser beiden Anteile in der RLZ durch das lokale elektrostatische Potential verändert wird:

- Am Rand der RLZ überwiegt zunächst der Anteil aus den Zuständen der Akkumulationszone (Bandmodell I in Abbildung 38 (b)).

- Das Ansteigen von $\phi_{R L Z}(x)$ bewirkt eine affine Verschiebung der Bänder, so dass das Energieintervall der besetzten Zustände im Leitungsband kleiner und das der besetzten Zustände im Valenzband, die zu den Tunnelstrom beitragen können, um denselben Betrag größer wird (Bandmodell II in Abbildung 38 (b)). Da die Zustände der Akkumulationszone gemäß (2-8) einen höheren Transmissionskoeffizienten besitzen nimmt der Anteil des Tunnelstroms aus der Akkumulationszone stärker ab, als der Anteil aus dem Valenzband zunimmt. Um den Setzstrom zu erreichen, muss der Tunnelabstand reduziert werden, so dass das steigende elektrostatische Potential in der RLZ anfangs zu einer Verringerung der relativen Spitzenhöhe führt.

- Erst wenn Zustände aus dem Valenzband den Tunnelstrom dominieren (Bandmodell III in Abbildung 38 (b)) nimmt der Abstand zwischen Spitze und Probe aufgrund des größer werdenden Energieintervalls wieder zu.

Die Analyse des elektronischen Kontrasts zeigt, dass die atomare Korrugation bei negativen BiasSpannungen am Rand der RLZ durch die an den Ga-Atomen lokalisierten Zustände bestimmt wird, wohingegen in der Nähe des Fe-Films die an den As-Atomen lokalisierten Zustände $A_{4}$ und $A_{5}$ den Tunnelstrom dominieren.

Neben der Bestimmung der Zustände, die bei den betrachteten Bias-Spannungen in Abhängigkeit von dem Abstand zur Metall-Halbleiter Grenzfläche zu dem Tunnelstrom beitragen, bieten die in Abbildung 37 und Abbildung 38 gezeigten Topografien erste Anhaltspunkte über die Weite der RLZ und die damit gemäß (1-5) zusammenhängende SBH des Systems. Es kann abgeschätzt werden, dass die Ausdehnung $w_{\text {STM }}$ des Bereichs, in dem veränderte Tunnelbedingungen beobachtet werden, im Durchschnitt etwa $40 \mathrm{~nm}$ beträgt. Ohne den Effekt des TIBB entspräche dies der Weite $w_{R L Z}$ der 
Raumladungszone (siehe auch Kapitel 7.1.2.5). Da das TIBB wegen des geringen Krümmungsradius der Tunnelspitze nur schwach ausgeprägt ist (vergleiche Kapitel 3.3.1), kann davon ausgegangen werden, dass sich $w_{R L Z}$ tatsächlich nur geringfügig von $w_{\text {STM }}$ unterscheidet. Unter der Annahme, dass die durchschnittliche Weite der RLZ darüber hinaus nur unwesentlich durch die vereinzelt auftretenden Oberflächendefekte verändert wird, kann gemäß Relation (1-5) abgeschätzt werden, dass die mittlere SBH des untersuchten Fe-GaAs(110) Schottky-Kontakts etwa $0.9 \mathrm{eV}$ beträgt. Auf eine quantitativere Bestimmung der SBH, bei der sowohl der Einfluss lokaler Defekte als auch der des TIBB berücksichtigt werden, wird in Kapitel 7.2 ausführlich eingegangen.

\subsection{Strukturelle Eigenschaften des Fe-GaAs(110) Schottky- Kontakts}

Ein zentraler Aspekt bei der Untersuchung des Fe-GaAs(110) Schottky-Kontakts ist die Bestimmung der lokalen atomaren Struktur im Bereich der Grenzfläche. Wichtige Gesichtspunkte sind hierbei die Homogenität der Grenzfläche, die Ausdehnung des Übergangsbereichs zwischen Metall und Halbleiter sowie die Bestimmung von charakteristischen grenzflächennahen Defekten, die mit der Deposition des Metallfilms zusammenhängen. Im Zusammenhang mit der Analyse der lokalen elektronischen Struktur in Kapitel 7 ist darüber hinaus die Bestimmung der diskreten Verteilung von Dotieratomen und anderen elektrisch aktiven Defekten in der RLZ von besonderem Interesse.

Um entsprechend die atomare Struktur vom Bereich der RLZ bis hin zur Fe-GaAs(110) Grenzfläche bestimmen zu können, eignen sich insbesondere hochaufgelöste XSTM Topografien, die bei unterschiedlichen Probenspannungen im Multi-Bias Modus aufgenommen wurden. Durch die Verwendung dieses Messmodus wird gewährleistet, dass die Topografien jeweils denselben Ausschnitt der Probenoberfläche bei verschiedenen Tunnelbedingungen zeigen (siehe Kapitel 2.3). In Kapitel 6.3 wurde gezeigt, dass durch die Verwendung von positiven und negativen Bias-Spannungen im Bereich der Grenzfläche das Ga- und das As-Untergitter räumlich zusammenhängend abgebildet werden können. Eine solche Multi-Bias Messung aus dem Bereich der Grenzfläche ist in Abbildung 39 dargestellt. Topografie (a) wurde bei einer Bias Spannung von 1,6 V abgebildet und zeigt im Bereich des GaAs Substrats das Ga-Untergitter. Dem gegenübergestellt wird in (b) das bei $U_{\text {Bias }}=-2,4 \mathrm{~V}$ abgebildete As-Untergitter. 
(a)

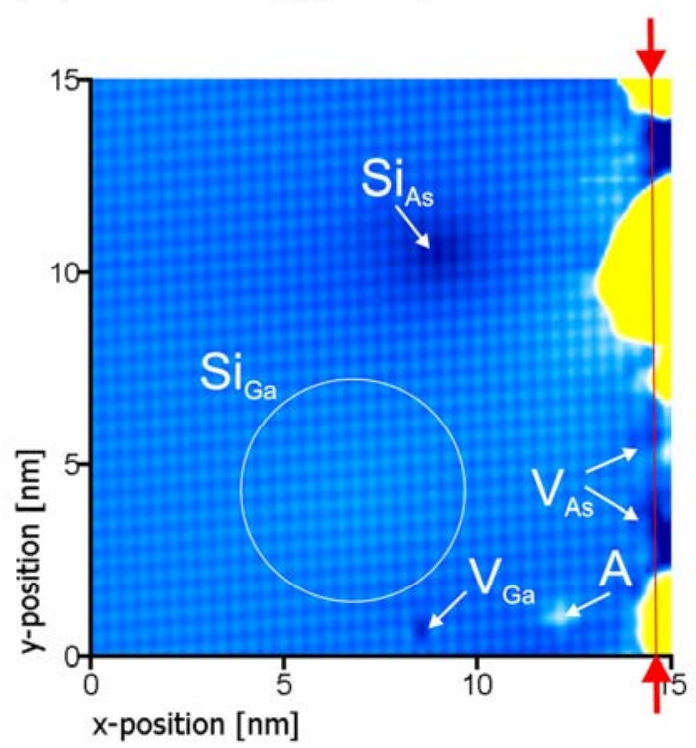

(b) $\quad U_{\text {Bias }}=-2,4 \mathrm{~V}$

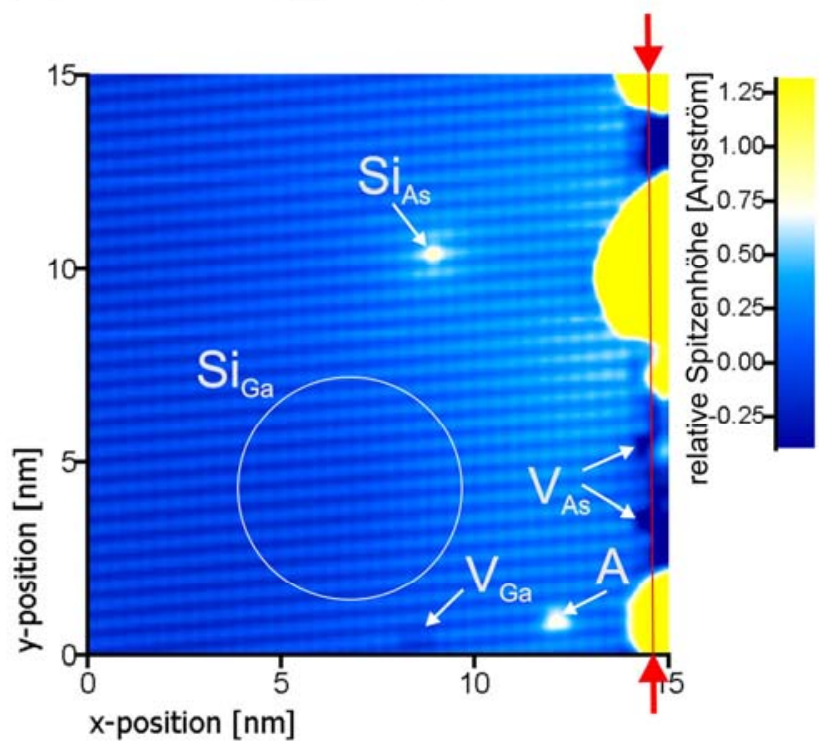

Abbildung 39: Atomar aufgelöste XSTM Topografien desselben Ausschnitts ((15 x 15) nm²), die im Multi-Bias Modus bei unterschiedlichen Setzpunkten aufgenommen wurden ((a) bei $U_{\text {Bias }}=1,6 \mathrm{~V}$, $I_{T}=100 \mathrm{pA}$, (b) bei $U_{\text {Bias }}=-2,4 \mathrm{~V}, I_{T}=100 \mathrm{pA}$ ). Der Verlauf der Grenzfläche wird jeweils durch die Verbindungslinie zwischen den roten Pfeilen markiert. Die Positionen von verschiedenen atomaren Defekten im Bereich der GaAs Spaltfläche sind gekennzeichnet.

Im Bereich des atomar glatt gespaltenen GaAs Substrats sind an verschiedenen Stellen elektronische Kontraste erkennbar, die auf unterschiedliche atomare Defekte im Kristallgitter zurückgeführt werden können. Der Einfluss, den die jeweiligen Defekte auf die STM Topografien haben, ist zum Teil sehr lokal, er kann aber auch über viele Oberflächeneinheitszellen ausgedehnt sein. Ein Vergleich der beiden in Abbildung 39 gezeigten Topografien macht deutlich, dass die Kontraste der Defekte darüber hinaus sehr stark von der jeweils verwendeten Bias-Spannung abhängen. In verschiedenen Arbeiten konnte gezeigt werden, dass dies als „Fingerabdruck“ verwendet werden kann, um atomare Defekte eindeutig zu identifizieren und um ihre Position im Kristallgitter zu bestimmen [63, 80, 129, 130]. Bei der Identifikation von Defekten, die sich innerhalb der RLZ des Schottky-Kontakts befinden, muss zusätzlich das lokale elektrostatische Potential berücksichtigt werden, durch das sich die Spannungsabhängigkeit der jeweiligen Signatur stark verändern kann. Dies kann dazu führen, dass ein Defekt bei einer festen Bias-Spannung in der Nähe der Metall-Halbleiter Grenzfläche einen anderen Kontrast verursacht als ein identischer Defekt am Rand der RLZ. Bei Defekten, deren Ladungszustand durch das elektrostatische Potential in der RLZ verändert wird, ist dieser Effekt besonders ausgeprägt. 


\subsubsection{Identifikation der Defekte}

Die Kontraste, die in den Topografien in Abbildung 39 markiert sind, können aufgrund ihrer bekannten Spannungsabhängigkeit sowie der Abstandsabhängigkeit zum Fe-Film auf die folgenden Defekte zurückgeführt werden:

$\mathbf{S i}_{\text {Ga }}$ : Einige atomare Lagen unterhalb der GaAs(110) Oberfläche befindet sich ein Si-Atom der Dotierung des n-GaAs Substrats, das substitutionell auf einem Ga-Gitterplatz eingebaut wurde [63]. Bei niedrigen bis moderaten Konzentrationen wird Si bevorzugt in dieser Konfiguration in GaAs eingebaut, in der es als flacher Donator wirkt [131]. Der Kontrast, den ein Si-Donator bei negativen Probenspannungen verursacht, ist stark von seiner Position in der RLZ abhängig. Um dies zu verdeutlichen, zeigt Abbildung 40 die Spannungsabhängigkeit der Kontraste von zwei Si-Donatoren, die sich in unterschiedlichen Abständen zur Metall-Halbleiter Grenzfläche befinden.

(a)

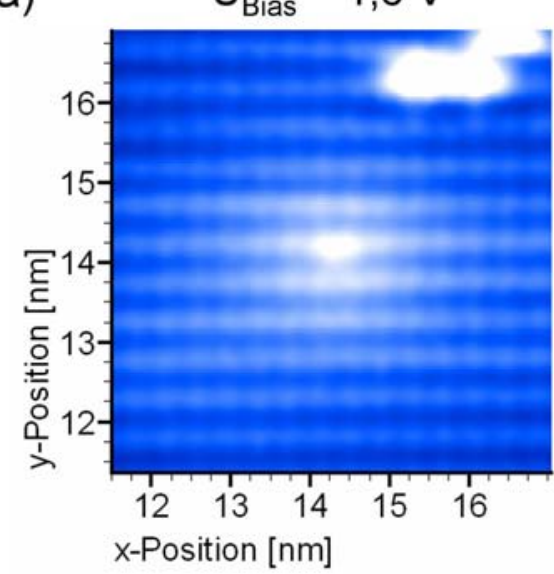

(b)

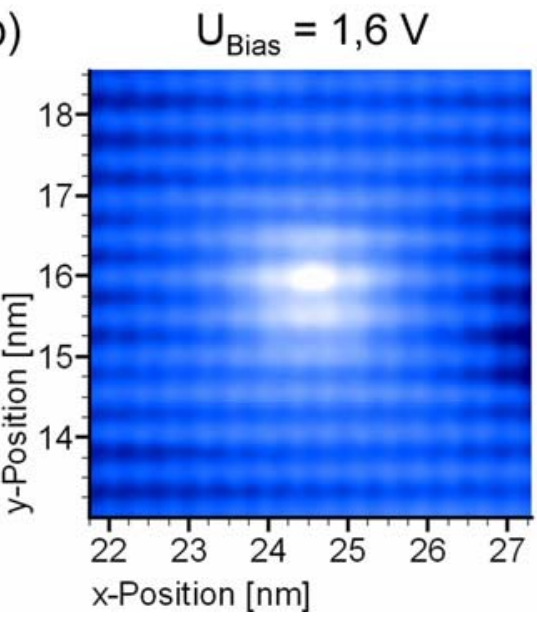

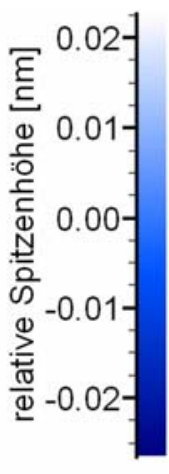

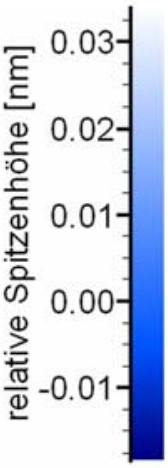

$$
U_{\text {Bias }}=-2,0 \mathrm{~V}
$$
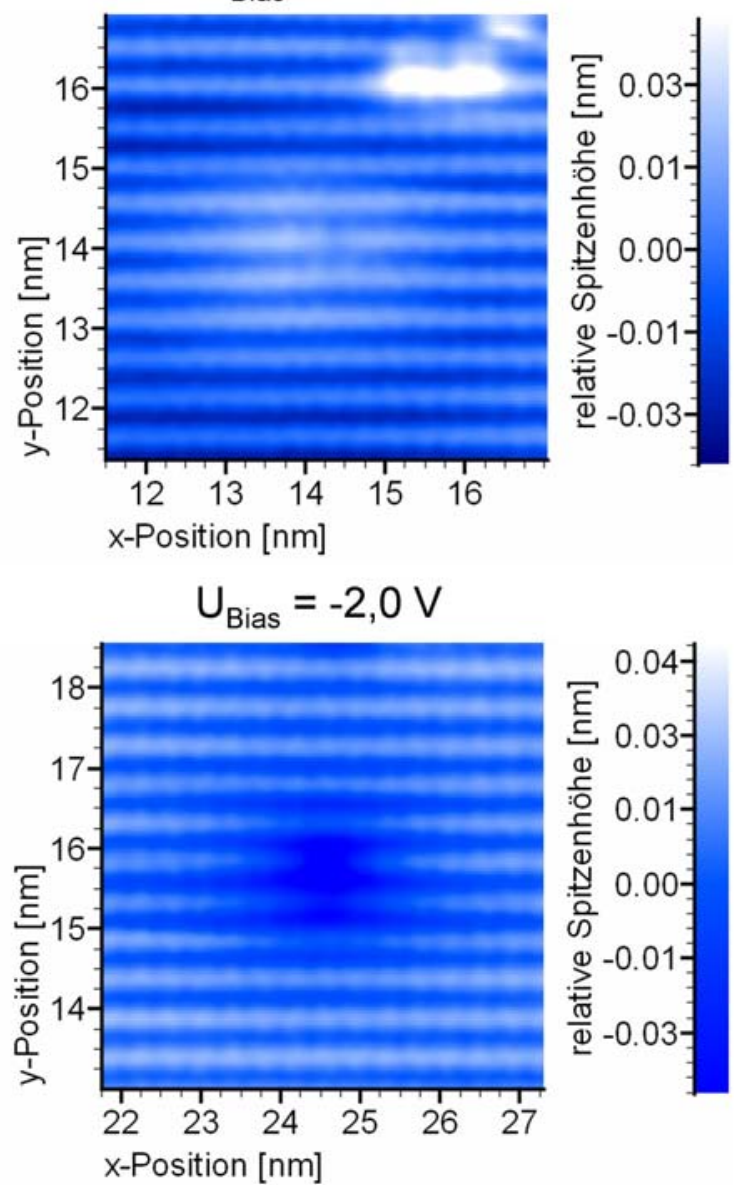

Abbildung 40: Spannungsabhängigkeit der Kontraste von zwei Si-Donatoren, die sich (a) etwa $14 \mathrm{~nm}$ und (b) etwa $3 \mathrm{~nm}$ von der Grenzfläche entfernt befinden. Die Topografien wurden im MultiBias Modus bei den angegebenen positiven und negativen Bias-Spannungen abgebildet. In (a) befinden sich Adatome in der rechten oberen Ecke, die allerdings keinen signifikanten Einfluss auf den Kontrast des Si-Donators haben. 
Während beide Donatoren bei einer positiven Bias-Spannung von 1,6 V unabhängig von ihrem Abstand zum Metallfilm nahezu identische Kontraste verursachen, ist bei der gezeigten negativen BiasSpannung von -2,0 V ein deutlicher Unterschied erkennbar. Abbildung 40 (a) zeigt, dass bis zu einem Abstand von etwa $15 \mathrm{~nm}$ zur Metall-Halbleiter Grenzfläche wie in der Literatur beschrieben eine Erhöhung beobachtet wird, die von einer leichten Vertiefung umgeben ist [100, 132]. Im Gegensatz dazu führt ein Si-Donator bei geringeren Abständen zu einer ausgedehnten Vertiefung in der Topografie. Abbildung 40 (b) verdeutlicht dies an einem oberflächennahen Donator, der sich in einem Abstand von etwa 3 nm zur Grenzfläche befindet. ${ }^{19}$

$\mathbf{S i}_{\mathbf{A s}}$ : In der Oberflächenlage kann ein Si-Dotieratom identifiziert werden, dass als Akzeptor substitutionell auf einem As-Gitterplatz eingebaut wurde [129]. Die Häufigkeit der $\mathrm{Si}_{\mathrm{As}}$-Akzeptoren ist generell geringer als die der $\mathrm{Si}_{\mathrm{Ga}}$-Donatoren. Bei der Dotierkonzentration des verwendeten Substrats kann davon ausgegangen werden, dass sich etwa $10 \%$ der Si-Dotieratome auf As-Gitterplätzen befinden [133].

$\mathbf{V}_{\text {Ga }}$ : Eine Ga-Fehlstelle in der Oberflächenlage ist bei positiver Bias-Spannung in Abbildung 39 (a) als lokalisierter Kontrast deutlich zu erkennen. Bei der Abbildung mit negativer Probenspannung (Abbildung 39 (b)) ist der Einfluss des Defekts auf die db-Zustände der direkt benachbarten As-Atome beschränkt. Der lokalisierte Kontrast bei negativer Probenspannung zeigt, dass der Defekt ungeladen ist. Aufgrund der spannungsabhängigen Signatur könnte es sich daher um einen Komplex aus einer Ga-Fehlstelle und einem Si-Donator handeln [129]. Die Auswertung verschiedener Topografien zeigt allerdings, dass offenbar auch der Ladungszustand einzelner Ga-Fehlstellen durch das elektrostatische Potential in der RLZ verändert wird. Ga-Fehlstellen, die sich in der Nähe der Metall-Halbleiter Grenzfläche befinden, sind generell ungeladen, wohingegen Fehlstellen, die sich am Rand bzw. außerhalb der RLZ befinden, in der Regel wie in der Literatur beschrieben geladen sind [63]. Diese Abhängigkeit des Kontrasts von der Position der Fehlstelle innerhalb der RLZ verdeutlichen die Topografien in Abbildung 41. Die beschriebene Veränderung des Kontrasts bei negativen Bias-Spannungen ist deutlich zu erkennen. Die Analyse der Kontraste zeigt, dass es sich bei dem mit $\mathrm{V}_{\mathrm{Ga}}$ bezeichneten Defekt um eine einzelne Ga-Fehlstelle handelt.

\footnotetext{
${ }^{19}$ Der Abstand zur Metall-Halbleiter Grenzfläche, bei dem die Änderung des Kontrasts beobachtet wird, ist von dem TIBB abhängig und daher nicht konstant. Er verringert sich mit steigenden negativen Spannungen.
} 
(a) $\quad U_{\text {Bias }}=1,8 \mathrm{~V}$

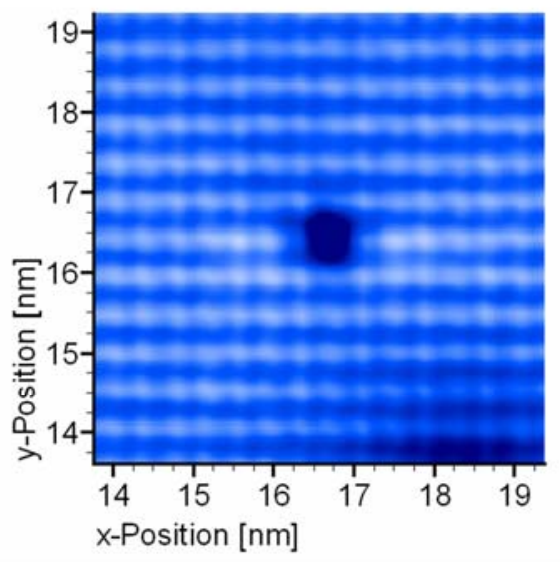

(b)

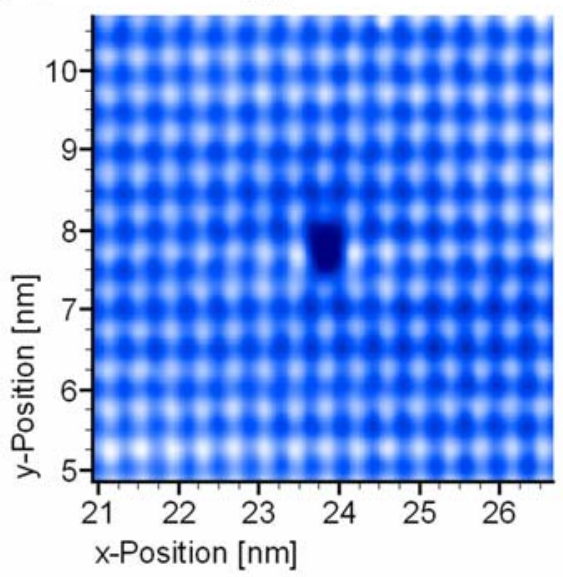

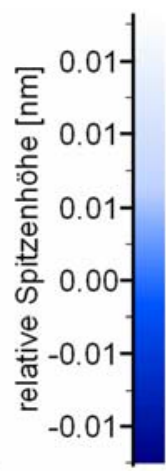

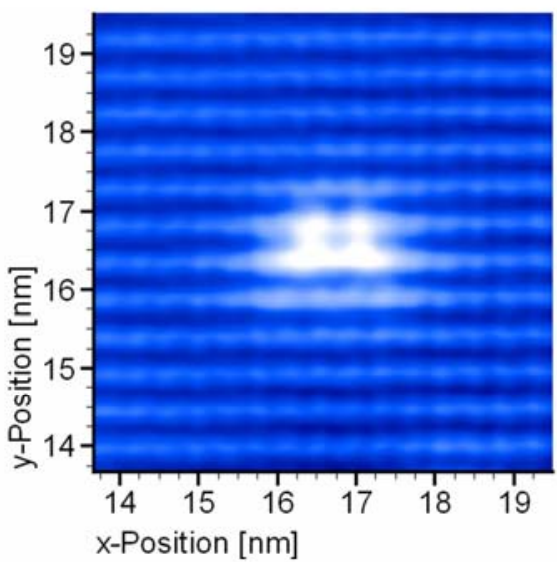

$$
U_{\text {Bias }}=-2,4 \mathrm{~V}
$$

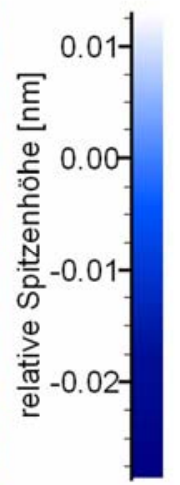

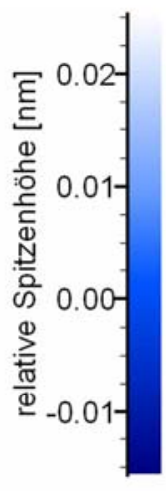
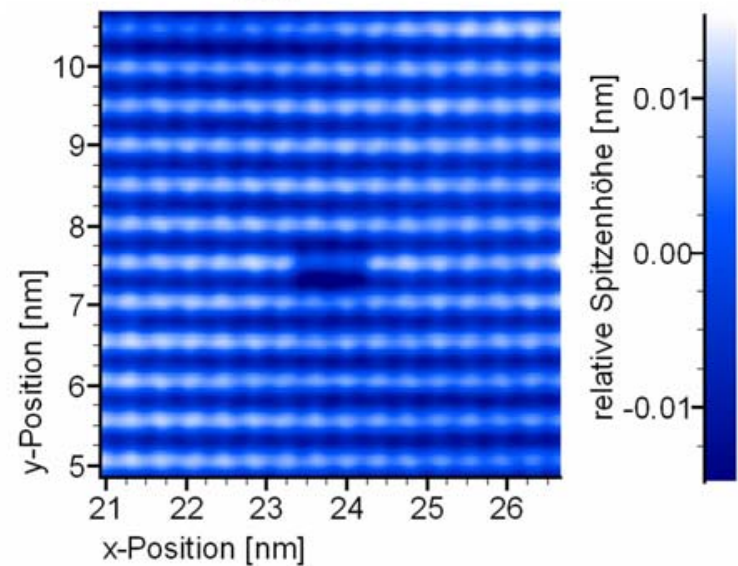

Abbildung 41: Spannungsabhängigkeit der Kontraste von zwei Ga-Fehlstellen, die sich (a) etwa $13 \mathrm{~nm}$ und (b) etwa $5 \mathrm{~nm}$ von der Grenzfläche entfernt befinden. Die Topografien wurden im MultiBias Modus bei den angegebenen positiven und negativen Bias-Spannungen abgebildet.

$\mathbf{V}_{\text {As: }}$ : Im Bereich der Grenzfläche können in Analogie zu der Ga-Fehlstelle zwei As-Fehlstellen in der Oberflächenlage identifiziert werden. Der Einfluss, den die Fehlstellen auf die Abbildung des Ga-Untergitters (Abbildung 39 (a)) besitzen, ist jeweils auf die db-Zustände der unmittelbar benachbarten Ga-Atome beschränkt.

A: Auf der GaAs(110) Spaltfläche befindet sich ein Adatom. Während der STM Messung verändern diese Adatome relativ häufig ihre Position, was von Ga-Adatomen bekannt ist [63]. In diesen Fällen wird der Bereich der GaAs(110) Oberfläche, der sich zuvor unter dem Adatom befunden hat, zugänglich und es kann gezeigt werden, dass die Oberfläche unter dem Adatom defektfrei ist. 


\subsubsection{Klassifizierung der Defekte}

Die Ausbildung von Oberflächendefekten, die mit der Erzeugung der Spaltflächen in Zusammenhang stehen, kann trotz des hohen präparativen Aufwands nicht ausgeschlossen werden. Nachdem die Defekte identifiziert wurden ist es daher notwendig, sorgfältig zu analysieren, welche der Defekte vor dem Spaltvorgang noch nicht vorhanden waren und dementsprechend nicht im Zusammenhang mit dem untersuchten Fe-GaAs(110) Schottky-Kontakt stehen. Die fraglichen Defekte in der Oberflächenlage sind:

$\mathbf{V}_{\mathbf{G a}}$, A: Von n-GaAs Substraten ist bekannt, dass Ga-Fehlstellen in der Oberflächenlage nach dem Spaltvorgang spontan durch Langmuir-Desorption entstehen [63]. Die freigesetzten Ga-Atome befinden sich zunächst als Adatome auf der Oberfläche bevor sie desorbieren. Sowohl die Anzahl der Ga-Fehlstellen als auch die der Adatome nimmt entsprechend mit der Zeit zu. Dieses Verhalten konnte auch bei der hier untersuchten Probe beobachtet werden. Da es sich bei der Ga-Fehlstelle und dem Adatom um Defekte handelt, die für n-GaAs Spaltflächen typisch sind, kann davon ausgegangen werden, dass sie durch den experimentellen Zugang hervorgerufen wurden.

$\mathbf{V}_{\text {As }}$ : Die As-Fehlstellen befinden sich sowohl in der Oberflächenlage als auch unmittelbar an der Metall-Halbleiter Grenzfläche. Als Ursache für diese Defekte kommt daher a) der erste Spaltvorgang, b) der deponierte Fe-Film oder c) der zweite Spaltvorgang in Frage.

a) Aus STM Untersuchungen von GaAs $\{110\}$ Spaltflächen ist bekannt, dass bei stark gestuften, defektreichen Oberflächen gelegentlich einzelne As-Fehlstellen in der Oberflächenlage auftreten können [134]. Es erscheint allerdings sehr unwahrscheinlich, dass bei dem ersten Spaltvorgang nur As-Fehlstellen erzeugt wurden, ohne dass auch vermehrt andere Defekte aufgetreten sind, für die es in Abbildung 39 aber keine Hinweise gibt. Es kann daher ausgeschlossen werden, dass die As-Fehlstellen mit dem ersten Spaltvorgang in Zusammenhang stehen.

b) In Kapitel 5.1 wurden verschiedene experimentelle Ergebnisse zusammengefasst, die übereinstimmend zeigen, dass es bei konventionell gewachsenen Fe-GaAs\{110\} Schottky-Kontakten generell zu Reaktionen an der Grenzfläche kommt. In verschiedenen Arbeiten wurde berichtet, dass sich im Bereich des Metall-Halbleiter Übergangs As- und Ga-haltige Fe-Phasen bilden und es zu einer Segregation von As-Atomen an die Oberfläche des Fe-Films kommt [44-46, 116, 119]. Auch in dem in Kapitel 1.5 vorgestellten UDM/AUDM wird davon ausgegangen, dass es durch die Deposition von Metallatomen auf Halbleitersubstraten generell zur Ausbildung von grenzflächennahen Defekten kommt, die für das FLP der Schottky-Kontakte verantwortlich gemacht werden [9, 10, 62]. Aufgrund der Erkenntnisse von konventionell gewachsenen Fe-GaAs\{110\} SchottkyKontakten kann nicht ausgeschlossen werden, dass die As-Fehlstellen durch den Metallfilm verursacht wurden, obwohl der untersuchte Fe-GaAs(110) Schottky-Kontakt bei LT deponiert wurde. Aus selbstkonsistenten DFT-Rechnungen der relaxierten Fe-GaAs Grenzflächenstruktur, die sehr 
gut mit den in Kapitel 5.1 zusammengefassten experimentellen Erkenntnissen des epitaktischen Wachstums von Fe-GaAs Systemen übereinstimmen, geht hervor, dass sich Fe-Atome als Folge von Reaktionen an der Grenzfläche bevorzugt auf einem Zwischengitterplatz oder substitutionell auf einem Ga-Gitterplatz befinden [48, 49]. TEM Untersuchungen von Fe-GaAs(001) Grenzflächen bestätigen die theoretischen Ergebnisse [47, 57]. Da im Fall der in Abbildung 39 gezeigten Topografien keine derartigen Defekte beobachtet werden, kann davon ausgegangen werden, dass eine Durchmischung des Grenzflächenbereichs effektiv unterdrückt werden konnte. Die beiden As-Fehlstellen stehen demnach in keinem kausalen Zusammenhang mit dem Wachstum des FeFilms.

c) In Kapitel 6.1 wurde dargelegt, dass es während des zweiten Spaltvorgangs im Bereich des Fe-Films zu starken strukturellen Veränderungen gekommen ist. Aufgrund von Fe-As- bzw. FeGa-Bindungen an der Grenzfläche kann davon ausgegangen werden, dass dies auch Auswirkungen auf den angrenzenden Bereich des GaAs Substrats gehabt hat. Es ist daher wahrscheinlich, dass es durch das Spaltverhalten des Fe-Films zu einer vermehrten Ausbildung von Defekten an der Grenzfläche gekommen ist. Die Tatsache, dass in Abbildung 39 nur As-Fehlstellen beobachtet werden, kann in diesem Zusammenhang durch die stärkere Fe-As-Bindung erklärt werden [49].

Bei allen Oberflächendefekten, die durch ihre Kontraste in Abbildung 39 identifiziert werden konnten, handelt es sich demnach um Defekte, die mit der Erzeugung der zweiten Spaltfläche und nicht mit dem untersuchten Fe-GaAs(110) Schottky-Kontakt im Zusammenhang stehen.

\subsubsection{Atomare Struktur des Fe-GaAs(110) Schottky-Kontakts}

Abgesehen von den beiden As-Fehlstellen ist der Verlauf der Grenzfläche des Fe-GaAs(110) SchottkyKontakts, der in Abbildung 39 durch die Verbindungslinie zwischen den roten Pfeilen markiert wird, abrupt und atomar glatt. Dies zeigt, dass es bei dem ersten Spaltvorgang gelungen ist, eine ideale planare Substratoberfläche zum Wachstum des Schottky-Kontakts zu präparieren. Aus dem lateralen Versatz der Korrugationsmaxima des As-Untergitters relativ zu den Maxima des Ga-Untergitters, der anhand der Multi-Bias Topografien in Abbildung 39 bestimmt werden kann, kann die Anordnung der Ga- und As-Atome an der Metall-Halbleiter Grenzfläche identifiziert werden. Eine Skizze der atomaren Struktur des Fe-GaAs(1110) Schottky-Kontakts zeigt Abbildung 42. ${ }^{20}$

\footnotetext{
${ }^{20}$ Die Darstellung ist stark vereinfacht und soll lediglich die Abfolge von As- und Ga-Atomen im Bereich der Grenzfläche illustrieren.
} 


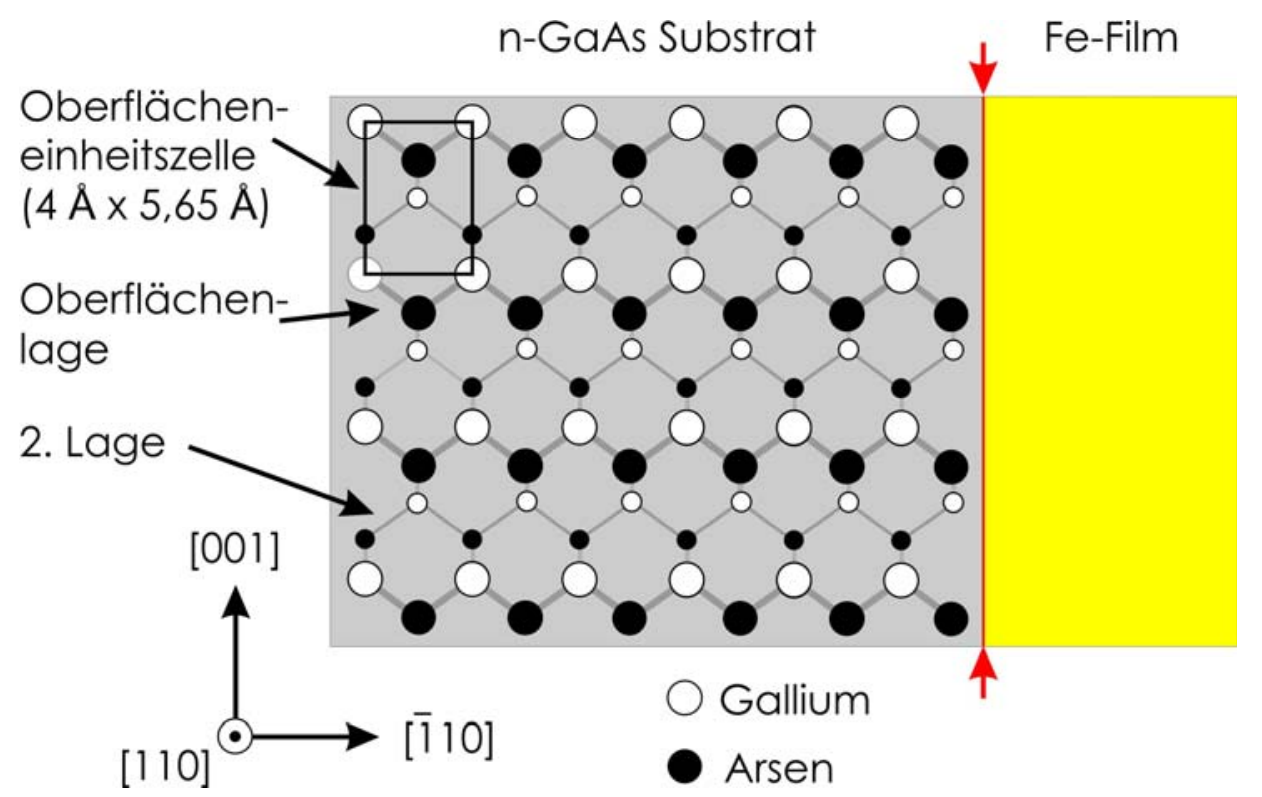

Abbildung 42: Schematische Darstellung der Struktur des Fe-GaAs(110) Schottky-Kontakts im Bereich der Grenzfläche. Die Zick-Zack-Reihen der Oberflächenlage enden an der Metall-Halbleiter Grenzfläche jeweils mit einem As-Atom.

Aus den in Abbildung 39 gezeigten XSTM Topografien geht hervor, dass die auf den Fe-Film zulaufenden Zick-Zack-Reihen der GaAs(110) Oberflächenlage an der Grenzfläche stets in derselben (110) Ebene enden. Es handelt sich bei dieser Ebene um die Spaltfläche, die durch den ersten Spaltvorgang erzeugt wurde. Wie in Abbildung 42 gezeigt ist das letzte Atom in der Oberflächenlage jeweils ein As-Atom. Es ist davon auszugehen, dass in dem in Abbildung 39 gezeigten Ausschnitt zwei dieser As-Atome während des zweiten Spaltvorgangs herausgerissen wurden.

Abgesehen von den beiden Si-Atomen der Dotierung, die sich als Donator bzw. Akzeptor innerhalb der RLZ des Schottky-Kontakts befinden und als systemspezifische Defekte die Eigenschaften des Fe-GaAs(110) Schottky-Kontakts lokal beeinflussen, konnten insbesondere im Bereich der Grenzfläche keine Defekte identifiziert werden, die mit dem Wachstum des Fe-Films in Verbindung stehen. 


\section{Lokale elektronische Eigenschaften: XSTS}

Durch die Verbindung von Querschnitts-Raster-Tunnel-Mikroskopie und -Spektroskopie können neben der atomaren Struktur auch die elektronischen Eigenschaften eines SchottkyKontakts im Ortsraum auf der atomaren Skala untersucht werden. In Kapitel 1 wurde bereits erläutert, dass die elektronischen Eigenschaften mit der Existenz von Bandlückenzuständen an der Grenzfläche zu dem Metallfilm in Zusammenhang gebracht werden, die zu dem Phänomen des „Fermi-level pinning“ führen. Um die diskutierten Ursachen des FLP kritisch überprüfen zu können, spielt neben der Charakterisierung dieser Zustände auch die Bestimmung der Schottky-Barrierenhöhe eine zentrale Rolle. Darüber hinaus bieten die XSTS Messungen die Möglichkeit, den Einfluss der diskreten Verteilung von ionisierten Dotieratomen auf die Potentiallandschaft in der RLZ zu untersuchen [27, 28].

$\mathrm{Zu}$ Beginn des Kapitels wird zunächst auf die I(U)-Spektroskopie im Bereich der RLZ des Fe-GaAs(110) Schottky-Kontakts eingegangen. Die charakteristischen Veränderungen in den I(U)Kennlinien, die in Abhängigkeit von dem Abstand zum Metallfilm beobachtet werden, werden im weiteren Verlauf des Kapitels sowohl im Rahmen von Tunnelstromsimulationen als auch im Rahmen von 3D-Simulationen der elektrostatischen Potentiallandschaft diskutiert. In Kapitel 7.2 wird erläutert, wie aus den experimentellen Daten und der 3D-Simulation des TIBB die Schottky-Barrierenhöhe sowie das lokale elektrostatische Potential in der RLZ extrahiert werden kann. Auf die Identifikation und Charakterisierung der Grenzflächenzustände wird in Kapitel 7.3 eingegangen.

Um die elektronischen Eigenschaften des Fe-GaAs(110) Schottky-Kontakts im Hinblick auf die verschiedenen angesprochen Gesichtspunkte untersuchen zu können, wurden Raster-TunnelSpektroskopie (STS) Messungen mit hoher räumlicher Auflösung durchgeführt. Ein STS-Datensatz besteht aus einer Topografie, einem Satz von I(U)-Kennlinien (siehe Kapitel 2.4), die an jedem Messpunkt der Topografie aufgezeichnet wurden, sowie der lokalen effektiven Barrierenhöhe $\phi_{B}$ (siehe Kapitel 2.5), die ebenfalls an jedem Messpunkt der Topografie aufgezeichnet wurde. Bei den STSMessungen entspricht der Setzpunkt der Spektroskopien dem Setzpunkt der Topografie, so dass der Tunnelabstand $d(x, y)$ zwischen Spitze und Probe, bei dem die Spektren aufgezeichnet wurden, aus der normierten Topografie hervorgeht. Um die I(U)-Kennlinien eines Datensatzes quantitativ miteinander vergleichen zu können, wurden die Spektren bei allen in dieser Arbeit gezeigten STS Messungen 
gemäß (2-1) mit dem lokal ermittelten $\phi_{B}$ auf einen einheitlichen Tunnelabstand $d$ normiert. ${ }^{21}$ Da bei der STS-Messung die spektroskopischen Daten zusammen mit der topografischen Information aufgezeichnet werden, können die lokalen elektronischen Eigenschaften direkt mit den strukturellen Gegebenheiten in Zusammenhang gebracht werden.

\subsection{I (U)-Spektroskopie in der Raumladungszone}

In Kapitel 6.3 wurde bereits gezeigt, dass in der RLZ des Schottky-Kontakts lokal variierende Tunnelbedingungen vorliegen. Die Auswirkungen können sowohl in XSTM Topografien beobachtet werden, in denen in Abhängigkeit vom Abstand zum Metallfilm ein ausgeprägter elektronischer Kontrast erkennbar ist, als auch bei der Untersuchung mit XSTS. Die charakteristischen Veränderungen, die bei den I(U)-Kennlinien in Abhängigkeit von dem Abstand zum Fe-Film beobachtet werden, verdeutlicht Abbildung 43.

\footnotetext{
${ }^{21}$ Das Verfahren kann angewendet werden, da die Messung von $\phi_{B}$ selbst nur in einem sehr geringem Maß von dem Tunnelabstand $d$ abhängt. In verschiedenen Arbeiten konnte demonstriert werden, dass die effektive Barrierenhöhe durch das Verringern des Tunnelabstandes $d$ um mehr als 1 A nicht signifikant verändert wird $[95,145]$.
} 
(a)

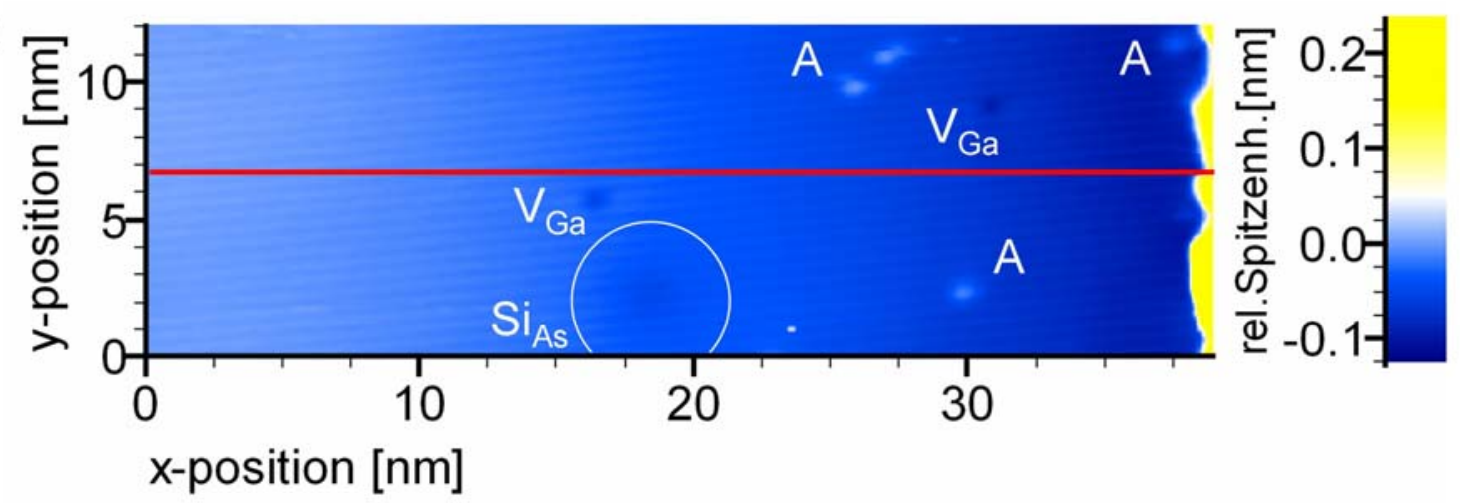

(b)

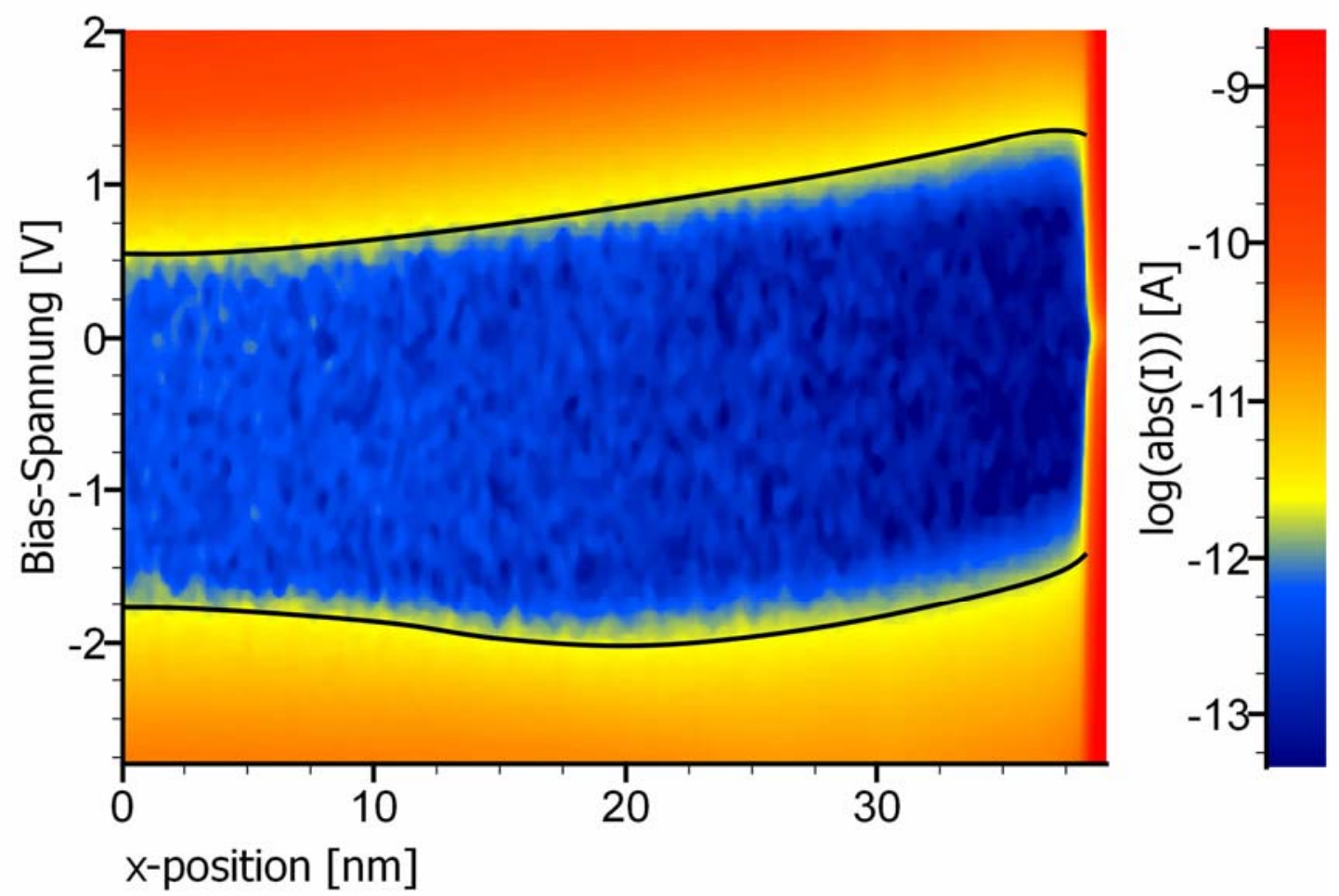

Abbildung 43: (a) Normierte XSTM Topografie aus dem Bereich der RLZ (etwa (39 x 12) $\mathrm{nm}^{2}$, $\left.U_{\text {Bias }}=2,0 \mathrm{~V}, I_{T}=100 \mathrm{pA}\right)$. Am rechten Rand ist der Fe-Film zu erkennen. (b) I(U)-Spektroskopie entlang der roten Linie in (a). Die Farbskala zeigt den Betrag von $I_{T}$ in einer logarithmischen Darstellung als Funktion der Probenspannung an den jeweiligen Messpunkten. Parallel zur Grenzfläche wurde jeweils über 3 Spektren gemittelt.

Die XSTM Topografie in Abbildung 43 (a) zeigt den Bereich der RLZ, der mit STS untersucht wurde. Neben verschiedenen lokalisierten Kontrasten, die von Adatomen, Ga-Fehlstellen und einem Si-Akzeptor verursacht werden, ist der elektronische Kontrast der RLZ deutlich erkennbar (vergleiche Abbildung 37). Die rote Linie markiert einen senkrecht auf die Metall-Halbleiter Grenzfläche zulaufenden Schnitt durch den Messbereich, der nur unwesentlich durch die strukturellen Defekte beeinflusst wird. Die STS Daten, die entlang dieses Schnittes aufgenommen wurden, sind in Abbildung 43 (b) dargestellt. Die Abbildung setzt sich aus insgesamt 250 aneinander gereihten I(U)-Kennlinien zu- 
sammen, bei denen der Logarithmus des Tunnelstroms jeweils farbkodiert dargestellt ist. Da die Metall-Halbleiter Grenzfläche bei dem gezeigten Datensatz durch das Spitzenartefakt nicht direkt zugänglich ist, wird dieser Bereich gesondert in Kapitel 7.3 diskutiert.

Bei den Spektren aus dem Bereich der RLZ existiert aufgrund der Bandlücke des Halbleiter Substrats ein Spannungsintervall, in dem der Tunnelstrom unter das Grundrauschen der Messapparatur von etwa 1 pA fällt. Sobald bei positiven bzw. negativen Bias-Spannungen Tunnelprozesse in die bandartigen Zustände des Substrats möglich werden, ist bei den I(U)-Kennlinien ein deutlicher Anstieg des Tunnelstroms erkennbar. Die Bias-Spannung, bei der ein gut messbarer Tunnelstrom von 3 pA beobachtet wird, wird im Folgenden als Einsatzpunkt bezeichnet. Sie ist stark davon abhängig, in welchem Abstand zum Metallfilm die Spektren aufgenommen wurden. Die in Abbildung 43 (b) eingezeichneten schwarzen Linien geben die Tendenzen wider:

- Bei positiven Bias-Spannungen verschiebt sich der Einsatzpunkt mit geringer werdendem Abstand zum Metallfilm zunächst zu immer höheren Spannungen. Im Abstand von einigen Nanometern zur Metall-Halbleiter Grenzfläche kehrt sich die Tendenz um und es wird ein Absinken des Einsatzpunktes beobachtet.

- Bei negativen Bias-Spannungen verschiebt sich der Einsatzpunkt mit geringer werdendem Abstand zum Metallfilm zunächst zu größeren negativen Spannungen, bevor ab einem Abstand von etwa 15 nm zur Metall-Halbleiter Grenzfläche ein Absinken des Einsatzpunktes beobachtet wird. Kurz vor der Grenzfläche ist eine verstärkte Abnahme erkennbar.

Für diese charakteristischen Veränderungen in den I(U)-Kennlinien kommen verschiedene physikalische Ursachen in Frage, die in den folgenden Kapiteln im Detail diskutiert werden.

\subsubsection{Einfluss des elektrostatischen Potentials in der RLZ: 1D- Simulation von I(U)-Kennlinien}

Um in einem ersten Schritt die Auswirkungen von $\phi_{R L Z}(x)$ auf die XSTS Messung nachvollziehen zu können, bietet das in Kapitel 3.3.2 vorgestellte Programm IVCHAR die Möglichkeit, eine elektrostatische Offsetenergie $\phi_{\text {Offset }}$ in die Simulation von I(U)-Kennlinien mit einzubeziehen [93, 98, 99]. Da die Simulationen in einer 1D-Näherung durchgeführt werden, kann auf diesem Weg keine quantitative Übereinstimmung zwischen den theoretisch berechneten und den experimentellen I(U)-Kennlinien erreicht werden. Es ist allerdings möglich, durch die Simulationen tendenzielle Veränderungen in den XSTS Daten zu identifizieren, die durch $\phi_{R L Z}(x)$ hervorgerufen werden. In Abbildung 44 ist zunächst schematisch dargestellt, wie $\phi_{\text {Offset }}$ den Verlauf der Bandkanten modifiziert. 


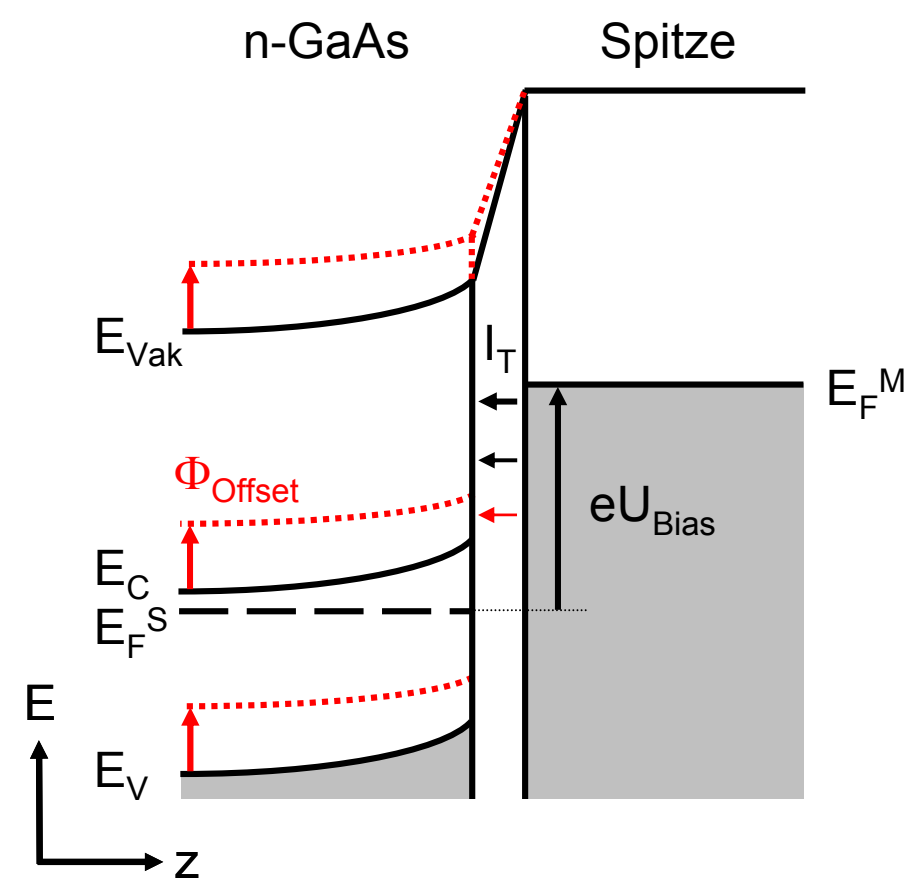

Abbildung 44: Skizze der Veränderungen, die durch $\phi_{\text {Offset }}$ bei einer positiven Bias-Spannung hervorgerufen werden. In dem 1D-Bandmodell werden die Bandkanten im Volumen des Substrats durch $\phi_{\text {Offset }}$ relativ zur Fermi-Energie affin verschoben.

Im Fall einer elektrostatischen Offsetenergie wird der Potentialverlauf selbstkonsistent für eine Situation berechnet, in der die Volumenbandstruktur des Halbleiters wie in Abbildung 44 skizziert um $\phi_{\text {Offset }}$ relativ zur Fermi-Energie affin verschoben ist. In dem gezeigten Beispiel nimmt sowohl der Potentialabfall über der Vakuumbarriere als auch im Substrat ab. Die energetische Lage der Bandkanten an der Oberfläche des GaAs Substrats verschiebt sich zu höheren Energien, so dass unter anderem bei derselben positiven Bias-Spannung ein geringerer Tunnelstrom berechnet wird.

Um derartige Modifikationen in den simulierten I(U)-Kennlinien, die in diesem Fall ausschließlich durch das zusätzliche $\phi_{\text {Offset }}$ hervorgerufen werden, mit den Veränderungen in den experimentellen Spektren in Abbildung 43 (b) vergleichen zu können, können Simulationen für ansteigende $\phi_{\text {Offset }}(x)$ durchgeführt werden. In Analogie zu dem experimentellen Spektrenschnitt zeigt Abbildung 45 (a) simulierte I(U)-Kennlinien, bei denen das in Abbildung 45 (b) dargestellte $\phi_{\text {Offset }}(x)$ vorgegeben wurde. $\phi_{\text {Offset }}(x)$ ist in diesem Fall identisch mit einem $\phi_{R L Z}(x)$, das in Anlehnung an die grobe Bestimmung der SBH in Kapitel 6.3 gemäß (1-5) und (1-6) für eine SBH von 0,9 eV und eine Si-Dotier- 
konzentration von $8 \cdot 10^{17} 1 / \mathrm{cm}^{3}$ berechnet wurde. Der rechte Rand in Abbildung 45 (a) entspricht der Position der Metall-Halbleiter Grenzfläche.

(a)

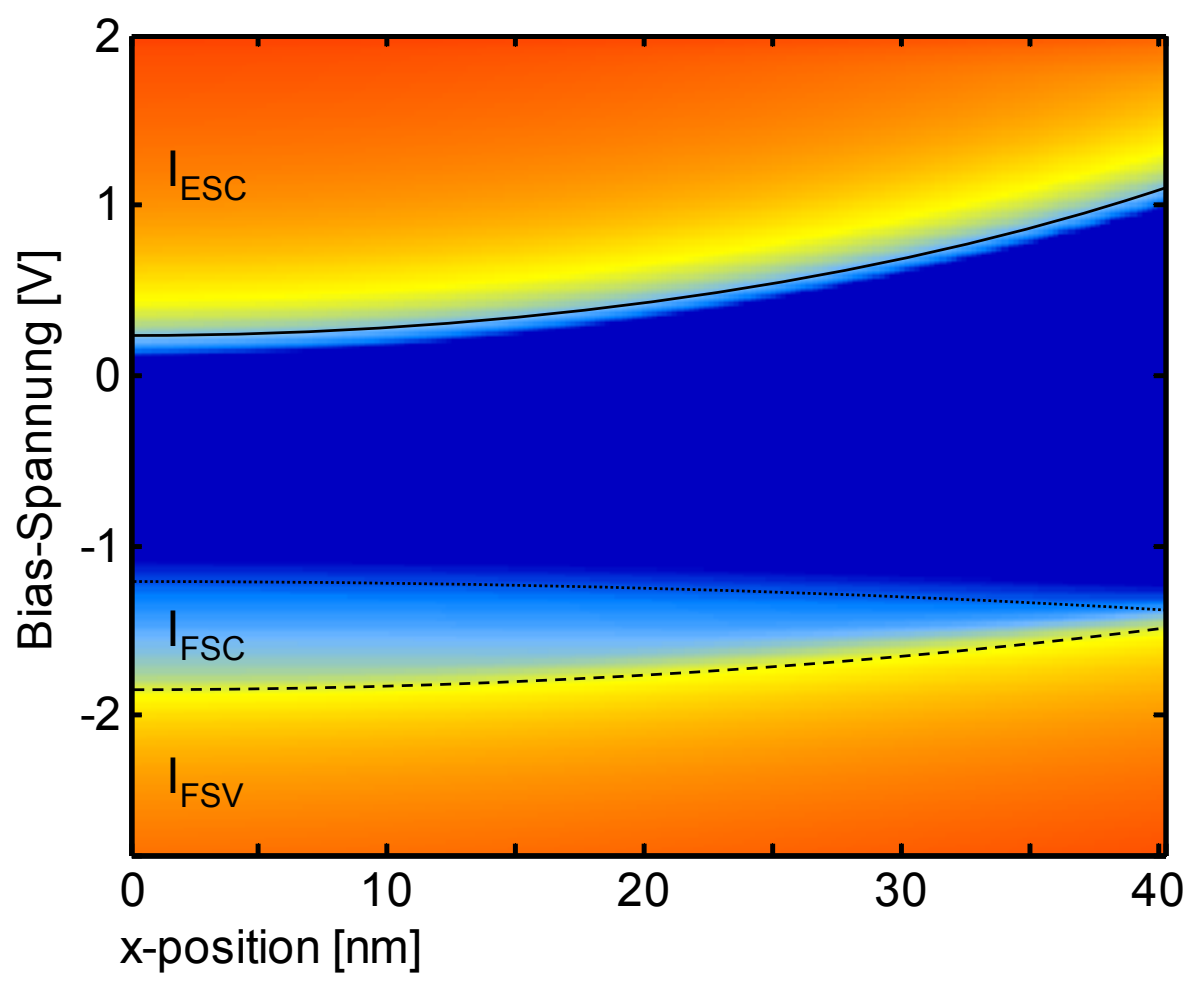

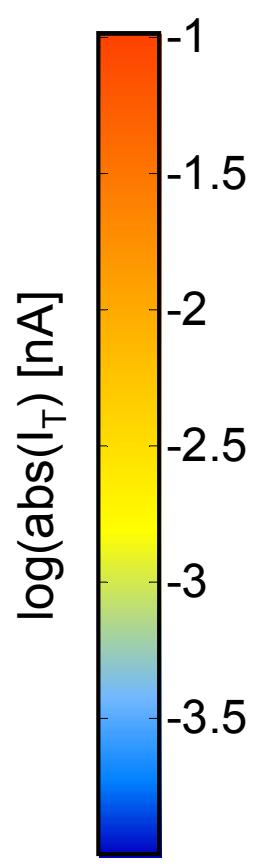

(b) $\underset{\circlearrowright}{>}$

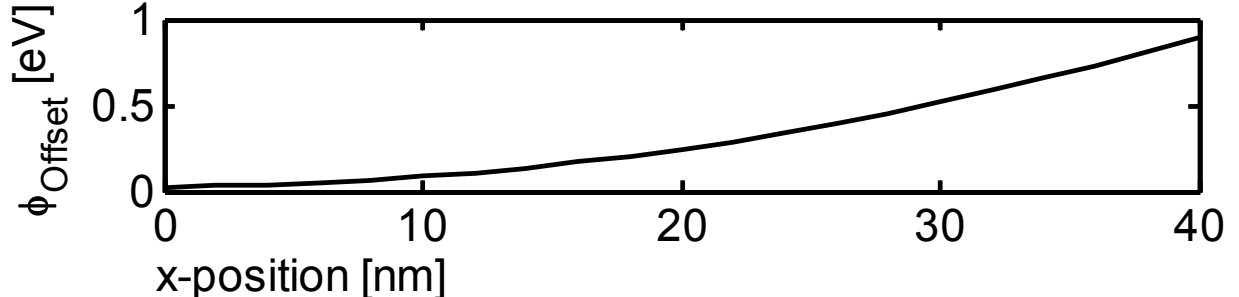

Abbildung 45: (a) Mit IVCHAR simulierte I(U)-Kennlinien, die für das in (b) gezeigte ansteigende $\phi_{\text {Offset }}(x)$ berechnet wurden. Der Logarithmus des Tunnelstroms ist jeweils farbkodiert dargestellt. Die zu dem Gesamttunnelstrom beitragenden Tunnelkanäle sind eingezeichnet. Die übrigen Parameter der Simulation sind bei allen I(U)-Kennlinien identisch (n-GaAs Substrat bei $293 \mathrm{~K}, 8 \cdot 10^{17} 1 / \mathrm{cm}^{3}$ Si dotiert, 0,8 nm Tunnelabstand, $4 \mathrm{eV}$ Spitzenaustrittsarbeit). (b) Elektrostatische Offsetenergie $\phi_{\text {Offset }}(x)$, die bei der Berechnung der I(U)-Spektren zugrunde gelegt wurde. Sie entspricht dem $\phi_{R L Z}(x)$ eines Schottky-Kontakts mit einer SBH von $0,9 \mathrm{eV}$.

Durch die Berücksichtigung von $\phi_{\text {Offset }}(x)$ werden bei der IVCHAR Simulation die folgenden tendenziellen Veränderungen in den I(U)-Kennlinien hervorgerufen: 
- Bei positiven Bias-Spannungen steigt mit steigendem $\phi_{\text {Offset }}(x)$ auch der Einsatzpunkt des Tunnelstroms kontinuierlich an. Es handelt sich hierbei um Tunnelprozesse in leere Leitungsbandzustände $\left(I_{E S C}\right)$.

- Im Fall einer festen positiven Bias-Spannung, z.B. 2 V, nimmt der Tunnelstrom bei gleich bleibendem Tunnelabstand $d$ mit steigendem $\phi_{\text {Offset }}(x)$ kontinuierlich ab.

- Bei negativen Bias-Spannungen kommt es für kleine $\phi_{\text {Offset }}(x)$ zunächst zu Tunnelprozessen aus besetzten Zuständen der Akkumulationszone $\left(I_{F S C}\right)$, die den Einsatzpunkt des Tunnelstroms bestimmen. Erst bei größeren negativen Bias-Spannungen tragen gefüllte Valenzbandzustände signifikant zu dem Tunnelstrom bei $\left(I_{F S V}\right)$. Mit steigendem $\phi_{O f f s e t}(x)$ steigt der Einsatzpunkt kontinuierlich an, da immer größere negative Bias-Spannungen notwendig werden, um eine Akkumulationszone zu erzeugen. Im Gegensatz dazu wird die Bias-Spannung, ab der $I_{F S V}$ zu dem Tunnelstrom beiträgt, immer geringer.

- Das Spannungsintervall, in dem aufgrund der GaAs Bandlücke keine Tunnelprozesse stattfinden können, wird mit steigendem $\phi_{\text {Offset }}(x)$ kontinuierlich größer.

Diese Tendenzen werden auch bei dem experimentellen Spektrenschnitt in Abbildung 43 (b) in einem weiten Bereich der RLZ beobachtet. Eine Ausnahme stellt der nur wenige Nanometer weite Bereich vor dem Fe-Film dar. Abgesehen von diesem Bereich können die Veränderungen in den I(U)Kennlinien wie folgt durch den Einfluss von $\phi_{R L Z}(x)$ auf die lokalen Tunnelbedingungen erklärt werden:

- Die bei positiven Bias-Spannungen auftretende Verschiebung des Einsatzpunktes zu größeren Spannungen wird dadurch verursacht, dass durch das ansteigende $\phi_{R L Z}(x)$ auch die Bandkanten an der Oberfläche des Substrats affin zu höheren Energien hin verschoben werden. Da bei den betrachteten positiven Bias-Spannungen von bis zu 2,0 V ausschließlich Tunnelprozesse in die unbesetzten Leitungsbandzustände des GaAs Substrats stattfinden (vergleiche Kapitel 3.3.2), müssen mit steigendem $\phi_{R L Z}(x)$ immer höhere Bias-Spannungen angelegt werden, bis ein Tunnelstrom zustande kommen kann. Es fällt allerdings auf, dass der Anstieg in den experimentellen Daten nicht exakt wie bei den simulierten I(U)-Kennlinien gemäß Relation (1-6) verläuft.

- Die bei negativen Bias-Spannungen beobachtete Verschiebung des Einsatzpunktes kann dadurch erklärt werden, dass zunächst $I_{F S C}$ am Rand der RLZ den Einsatzpunkt des Tunnelstroms bestimmt. Der Simulation entsprechend wird mit steigendem $\phi_{R L Z}(x)$ eine Verschiebung des Einsatzpunktes zu höheren negativen Bias-Spannungen beobachtet. Ab einem Abstand von etwa 
$15 \mathrm{~nm}$ zur Grenzfläche bestimmt hingegen $I_{F S V}$ den Einsatzpunkt, so dass der Tendenz aus der Simulation entsprechend wieder eine Verschiebung zu niedrigeren Spannungen stattfindet. Die Diskrepanz zwischen der Simulation und dem Experiment wird dadurch verursacht, dass das TIBB bei den Tunnelstromsimulationen nur in einer 1D-Näherung mit in die Berechnungen einbezogen wird. Dies hat unter anderem zur Folge, dass die Bias-Spannungen, ab denen $I_{F S C}$ und $I_{F S V}$ experimentell zum Gesamttunnelstrom beitragen, nicht realistisch wiedergegeben werden (vergleiche Kapitel 3.3.2).

Abgesehen von dem wenige Nanometer weiten Bereich vor dem Fe-Film können sowohl die Veränderungen bei positiven als auch bei negativen Bias-Spannungen in Verbindung mit den 1D-Simulationen einheitlich durch den Einfluss von $\phi_{R L Z}(x)$ auf die Tunnelbedingungen erklärt werden. Die Simulationen bestätigen darüber hinaus den in Kapitel 6.3 beschriebenen Zusammenhang zwischen $\phi_{R L Z}(x)$ und dem topografischen elektronischen Kontrast bei positiven und negativen Bias-Spannungen (siehe Abbildung 37 und Abbildung 38).

\subsubsection{Das TIBB in der Raumladungszone des Schottky-Kontakts: 3D- Simulation der elektrostatischen Potentiallandschaft}

In Kapitel 3.3.2 wurde erläutert, dass die im vorangegangenen Kapitel gezeigten IVCHAR Simulationen prinzipiell nur dann verlässliche Ergebnisse liefern können, wenn auch das TIBB im Rahmen der 1D-Näherung exakt berechnet werden kann. Bereits die Verwendung von STM Spitzen mit geringen Krümmungsradien bewirkt, dass die simulierten I(U)-Kennlinien generell nur qualitativ mit den experimentell gewonnenen Spektren übereinstimmen können. Sofern der Bereich der Probe homogen ist, in dem es zu dem TIBB kommt, kann der Fehler der IVCHAR Simulation durch die 3D-Berechnung des TIBB mit der von Feenstra entwickelten Methode abgeschätzt werden [97]. Bei der XSTM Untersuchung des Fe-GaAs(110) Schottky-Kontakts ist diese Voraussetzung allerdings nur weit außerhalb der RLZ gegeben. Schon am Rand der RLZ bewirkt das ansteigende elektrostatische Potential, dass das TIBB nicht mehr zufrieden stellend mit den bislang verfügbaren Methoden rekonstruiert werden kann. Das TIBB wird in diesem Fall neben der Bias-Spannung zusätzlich durch das lokale elektrostatische Potential beeinflusst. Dabei ist nicht nur $\phi_{R L Z}(x)$ am Ort der Spitze von Bedeutung, sondern der Verlauf des elektrostatischen Potentials in dem gesamten Bereich, in dem es zu dem TIBB kommt. Bei der IVCHAR Simulation kann dieser Zusammenhang nicht angemessen berücksichtigt werden, da durch $\phi_{\text {Offset }}(x)$ im Rahmen der 1D-Näherung lediglich simuliert werden kann, wie sich ein räumlich konstantes Offsetpotential auf die Tunnelbedingungen auswirkt. Mit geringer werdendem Abstand zur Metall-Halbleiter Grenzfläche muss darüber hinaus beachtet werden, dass die hohe Ladungsträgerkon- 
zentration des angrenzenden Metallfilms immer stärker zur Abschirmung des Potentials zwischen Spitze und Probe beiträgt und das TIBB entsprechend verändert. Da auch dieser Einfluss auf das TIBB bei der IVCHAR Simulation nicht berücksichtigt wird, treten mit abnehmendem Abstand zum Metallfilm immer größere Abweichungen zwischen den berechneten und den experimentellen I(U)Kennlinien auf (vergleiche Abbildung 43 (b) und Abbildung 45 (a)).

Eine über die IVCHAR Simulationen hinausgehende Analyse der XSTM und XSTS Daten aus dem Bereich der RLZ kann daher nur erreicht werden, wenn auch das TIBB den experimentellen Gegebenheiten entsprechend berechnet werden kann. Um dies zu erreichen wurde im Rahmen dieser Arbeit ein neues Simulationsverfahren entwickelt. Es basiert auf der Anwendung einer Finite Elemente Methode (FEM), mit der partielle Differentialgleichungen wie die Poisson-Gleichung (1-3) auch im Fall von komplexen, dreidimensionalen Problemen numerisch gelöst werden können. Dieser Ansatz besitzt keine der oben genannten konzeptionellen Einschränkungen, so dass das TIBB als Funktion des Ortes vom Rand der RLZ bis hin zur Metall-Halbleiter Grenzfläche simuliert werden kann, wobei alle experimentell bekannten Parameter wie z.B. der Krümmungsradius der Spitze oder die Dicke des Metallfilms berücksichtigt werden. ${ }^{22}$ Als Basis dient das kommerziell erhältliche Programmpaket COMSOL Multiphysics. Im Folgenden werden die wichtigsten Aspekte der Simulation des TIBB mit der Finite Elemente Methode erläutert.

\subsubsection{3D-Geometrie}

Ein großer Vorteil der Finite Elemente Methode besteht darin, dass die Geometrie des Berechnungsgebiets ohne Einschränkungen an das Experiment angepasst werden kann. Die Geometrie zur 3DSimulation des TIBB bei der XSTM Untersuchung des Fe-GaAs(110) Schottky-Kontakts ist in Abbildung 46 dargestellt. Die Aufteilung des Berechnungsgebiets ist der experimentellen Messanordnung nachempfunden und besteht aus der metallischen STM Spitze, die von Vakuum umgeben ist und sich im Tunnelabstand von wenigen Ångström vor der Metall-Halbleiter Spaltfläche befindet. Die Geometrie der Spitze wird über ihren Krümmungsradius und ihren Öffnungswinkel definiert. Ein weiterer relevanter Parameter der Simulationsgeometrie ist die Position der Spitze relativ zur MetallHalbleiter Grenzfläche. Darüber hinaus ist zu beachten, dass das Berechnungsgebiet ausreichend groß gewählt wird, um Artefakte durch die Randbedingungen zu vermeiden (siehe auch Kapitel 7.1.2.4).

\footnotetext{
${ }^{22}$ Den Ausführungen in Kapitel 7.1.3 entsprechend ist es dabei ausreichend, das bei positiven BiasSpannungen auftretende TIBB zu simulieren.
} 


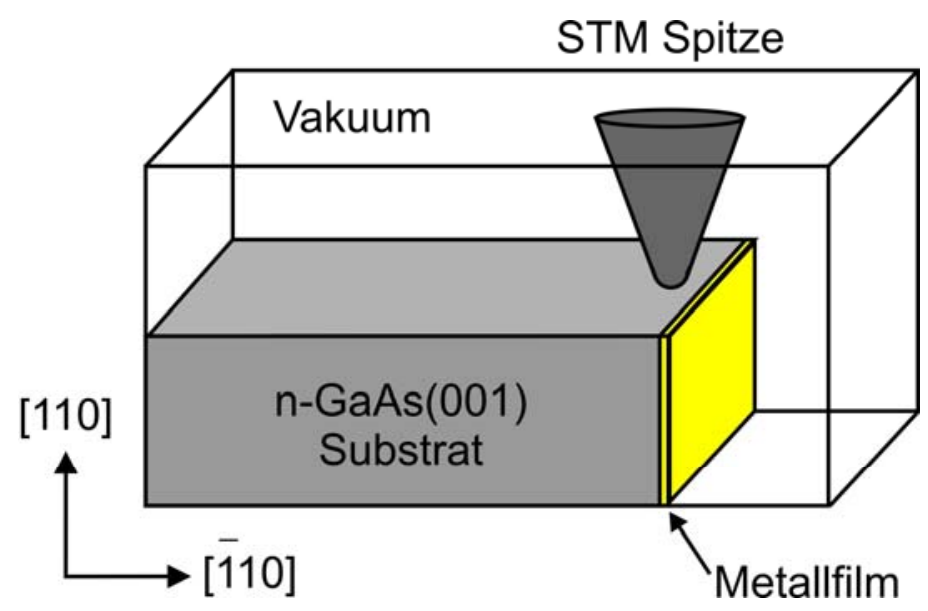

Abbildung 46: Geometrie zur Simulation der dreidimensionalen Potentiallandschaft. Entsprechend der Messanordnung während des XSTM Experiments befindet sich eine metallische Spitze im Vakuum nur wenige Ångström vor der zu untersuchenden Metall-Halbleiter Spaltfläche. Das Volumen des Berechnungsgebiets beträgt $(400 \times 500 \times 200) \mathrm{nm}^{3}$.

\subsubsection{Finite Elemente}

Das Prinzip der Finite Elemente Methode beruht darauf, dass das über die Geometrie definierte Berechnungsgebiet durch ein dreidimensionales Netz theoretisch in eine beliebige Anzahl von endlich großen Elementen unterteilt werden kann. Praktisch wird die Anzahl der Elemente durch die Rechenkapazität des verwendeten PC begrenzt. Bei den in dieser Arbeit durchgeführten Simulationen hat sich eine Anzahl von etwa $7 \cdot 10^{4}$ Elementen als praktikabel erwiesen, da bei einer feineren Unterteilung die Berechnungsdauer stark ansteigt. Artefakte durch die Diskretisierung können minimiert werden, indem die Maschenweite des Netzes insbesondere in den Bereichen des Tunnelkontakts, der spitzeninduzierten RLZ und der RLZ des Schottky-Kontakts reduziert wird, da hier die größten Potentialgradienten auftreten. Ein derart modifiziertes Netz ist in Abbildung 47 dargestellt. 


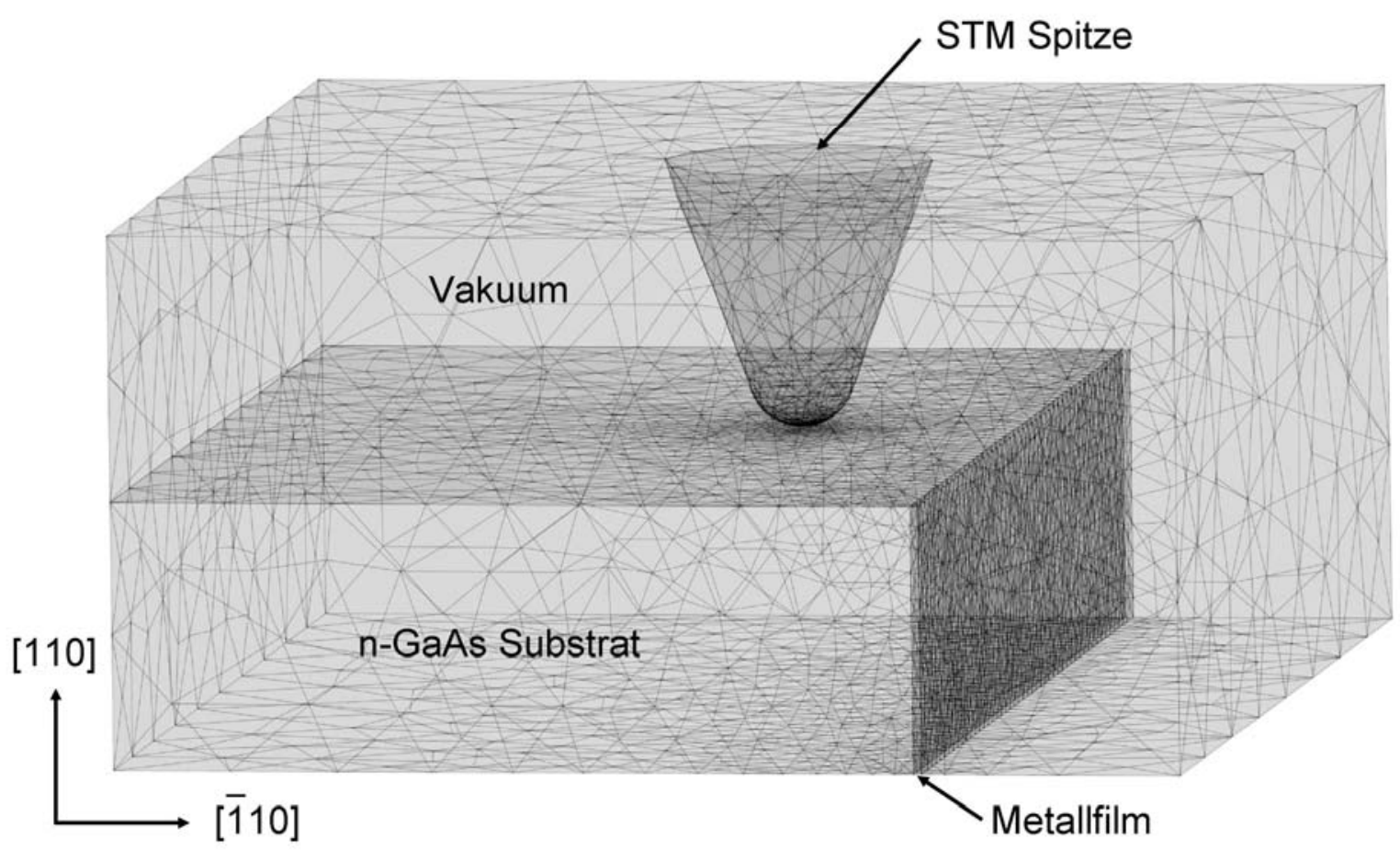

Abbildung 47: 3D-Netz, mit dem das Berechnungsgebiet in etwa $7 \cdot 10^{4}$ finite Elemente aufgeteilt wird. Die Maschenweite im Bereich des Tunnelkontakts und den Raumladungszonen von STM Spitze und Schottky-Kontakt wurde an die auftretenden Potentialgradienten angepasst.

Die lokale Verringerung der Maschenweite ist deutlich zu erkennen. Die Kantenlänge der Elemente im Bereich des Tunnelkontakts beträgt maximal $5 \AA$ und nimmt in der spitzeninduzierten RLZ von Element zu Element um maximal 2 Prozent zu. An der Metall-Halbleiter Grenzfläche wurde die Kantenlänge auf maximal $2 \mathrm{~nm}$ begrenzt.

\subsubsection{Berechnung der Ladungsdichte im Halbleiter Substrat}

Um die Poisson-Gleichung im Berechnungsgebiet numerisch lösen zu können, muss in jedem Element neben der Dielektrizitätskonstante die Ladungsdichte $\rho$ definiert werden. Im n-GaAs Substrat wird $\rho$ im Rahmen der Effektivmassen-Näherung durch die hier zusammengefassten Gleichungen beschrieben [135]. Für die Elektronendichte $n$ im Leitungsband und die Lochdichte $p$ im Valenzband des Halbleiters gilt:

$$
\begin{aligned}
& n=N_{C} \frac{2}{\sqrt{\pi}} F_{1 / 2}\left(\frac{E_{F}-E_{C}}{k T}\right), N_{C}=2\left(2 \pi m_{d e} k T / h^{2}\right)^{3 / 2} \\
& p=N_{V} \frac{2}{\sqrt{\pi}} F_{1 / 2}\left(\frac{E_{V}-E_{F}}{k T}\right), N_{V}=2\left(2 \pi m_{d h} k T / h^{2}\right)^{3 / 2}
\end{aligned}
$$


$\mathrm{E}_{\mathrm{F}}$ ist die Fermienergie, $\mathrm{E}_{\mathrm{C}}$ ist das Leitungsbandminimum, $\mathrm{E}_{\mathrm{V}}$ ist das Valenzbandmaximum, $\mathrm{T}$ ist die Temperatur, $k$ ist die Bolzmann-Konstante, $F_{1 / 2}$ ist das Fermi-Dirac-Integral der Ordnung 1/2, $N_{C}$ ist die effektive Zustandsdichte im Leitungsband mit der effektiven Elektronenmasse $\mathrm{m}_{\mathrm{de}}, \mathrm{N}_{\mathrm{V}}$ ist die effektive Zustandsdichte im Valenzband (entartetes Schwer- und Leichtlochband) mit der effektiven Lochmasse $m_{d h}$, h ist die Plank'sche Konstante. Der Faktor 2 in den Ausdrücken von $\mathrm{N}_{\mathrm{C}}$ und $\mathrm{N}_{\mathrm{V}}$ wird durch die Spin-Entartung hervorgerufen. Für die Dichte von ionisierten Donatoren gilt:

$$
N_{D}^{+}=\frac{N_{D}}{1+2 \exp \left(\frac{E_{F}-E_{D}}{k T}\right)}
$$

$\mathrm{N}_{\mathrm{D}}$ ist die Konzentration der Donatoren und $\mathrm{E}_{\mathrm{D}}$ ist die Ionisierungsenergie der Donatoren. Der Faktor 2 wird durch die Spin-Entartung bedingt.

Die Gesamtladungsdichte im n-GaAs Substrat setzt sich aus den einzelnen Beiträgen zusammen und ist entsprechend eine Funktion von $E_{F}$ :

$$
\rho\left(E_{F}\right)=e\left[p\left(E_{F}\right)+N_{D}^{+}\left(E_{F}\right)-n\left(E_{F}\right)\right]
$$

Im Volumen des Halbleiters kann die Lage von $E_{F}$ über die Bedingung $\rho\left(E_{F}\right) \stackrel{!}{=} 0$ bestimmt werden. Der entsprechende Wert von $E_{F}$ ist eine Funktion der Temperatur und wird als chemisches Potential $\mu_{c}$ bezeichnet.

Der Effekt des lokalen elektrostatischen Potentials $V(\vec{r})$ in der Raumladungszone auf die Ladungsdichte im Halbleiter wird im Rahmen einer semiklassischen Näherung (Rigid-Band-Modell) beschrieben. Es wird angenommen, dass $E_{F}$ im gesamten Bereich des Substrats konstant ist [97]. Die mit der Bandstruktur des Halbleiters assoziierten Energien $E_{C}, E_{V}$ und $E_{D}$ werden affin um $\phi(\vec{r})=-e V(\vec{r})$ relativ zu $E_{F}$ verschoben. Um die Ladungsdichte in der Raumladungszone zu erhalten, muss entsprechend $\rho\left(\mu_{c}-\phi(\vec{r})\right)$ berechnet werden.

\subsubsection{Randbedingungen}

Neben der Berechnung der Ladungsdichte im n-GaAs Substrat ist auch die Wahl der Anfangs-, Randund Übergangsbedingungen der Simulation entscheidend. Im Einzelnen sind dies:

- Die elektrostatische Energie $\phi_{T S}$ der Spitze relativ zum Halbleitersubstrat. $\phi_{T S}$ entspricht der Differenz zwischen dem Vakuumniveau im Bereich der Spitze und dem Vakuumniveau im Volumen des Halbleiters. Letzteres wird als Referenz auf Null gesetzt, so dass bei $\phi_{T S}=0$ Flachband- 
Bedingungen herrschen. $\phi_{T S}$ hängt über die folgende Beziehung mit der experimentellen BiasSpannung und den Austrittsarbeiten der Spitze $\left(\phi_{T}\right)$ und der Probe $\left(\phi_{S}=\chi_{S}+\left(E_{C}-\mu_{c}\right)\right)$ zusammen:

$$
\phi_{T S}=e U_{\text {Bias }}+\phi_{T}-\phi_{S}
$$

- Die elektrostatische Energie $\phi_{F S}$ des Metallfilms relativ zum Halbleitersubstrat. Sie entspricht der Differenz zwischen dem Vakuumniveau des Metallfilms und dem Vakuumniveau im Volumen des Halbleiters und hängt folgendermaßen mit der Schottky-Barrierenhöhe $\phi_{S B}$ zusammen:

$$
\phi_{F S}=\phi_{S B}+\left(E_{C}-\mu_{c}\right)
$$

- An den inneren Grenzflächen, z.B. zwischen Halbleiter und Vakuum, muss die Normalkomponente der elektrischen Flussdichte $\vec{D}=\varepsilon_{0} \varepsilon_{r} \vec{E}$ erhalten bleiben:

$$
\vec{n}\left(\vec{D}_{1}-\vec{D}_{2}\right)=0
$$

- An den äußeren Grenzflächen (ohne die Bereiche von Spitze und Metallfilm) wird die Randbedingung $\vec{n} \vec{D}=0$ gefordert (Neumann-Randbedingung). Da die Normalkomponente des elektrischen Feldes an den äußeren Grenzflächen in der Regel nicht verschwinden würde, stellt dies eine Näherung dar. Um ausschließen zu können, dass das zentrale Resultat der Simulation, d.h. das durch die STM Spitze verursachte TIBB, durch diese Näherung signifikant beeinflusst wird, wurden Simulationen für unterschiedlich große Berechnungsgebiete durchgeführt. Demnach ist das verwendete Berechnungsgebiet mit (400 x 500 x 200) $\mathrm{nm}^{3}$ ausreichend groß, so dass auch bei der Simulation von STM Spitzen mit Krümmungsradien von bis zu 40 nm Artefakte durch die Randbedingungen ausgeschlossen werden können.

\subsubsection{Simulation des TIBB in der Raumladungszone des Schottky-Kontakts}

Wie in Abschnitt 7.1.2.2 beschrieben ist die Ladungsdichte $\rho(\vec{r})$ im n-GaAs Substrat eine Funktion von $\phi(\vec{r})$, dessen räumlicher Verlauf berechnet werden soll. $\rho(\vec{r})$ und $\phi(\vec{r})$ müssen daher im Halbleiter in einem Iterationsverfahren unter Einhaltung der Rand- und Übergangsbedingungen selbstkonsistent berechnet werden. Abbildung 48 zeigt exemplarisch ein mit der Finite Elemente Methode berechneten Verlauf der elektrostatischen Energie in einer Ebene senkrecht zum Metallfilm. Die STM Spitze (Krümmungsradius: $20 \mathrm{~nm}$, Öffnungswinkel: $40 \mathrm{Grad}$ ) befindet sich 0,8 nm über der Probenoberfläche und wird zentral geschnitten. Der Abstand zwischen dem Apex der Spitze und der MetallHalbleiter Grenzfläche beträgt $80 \mathrm{~nm}$. 


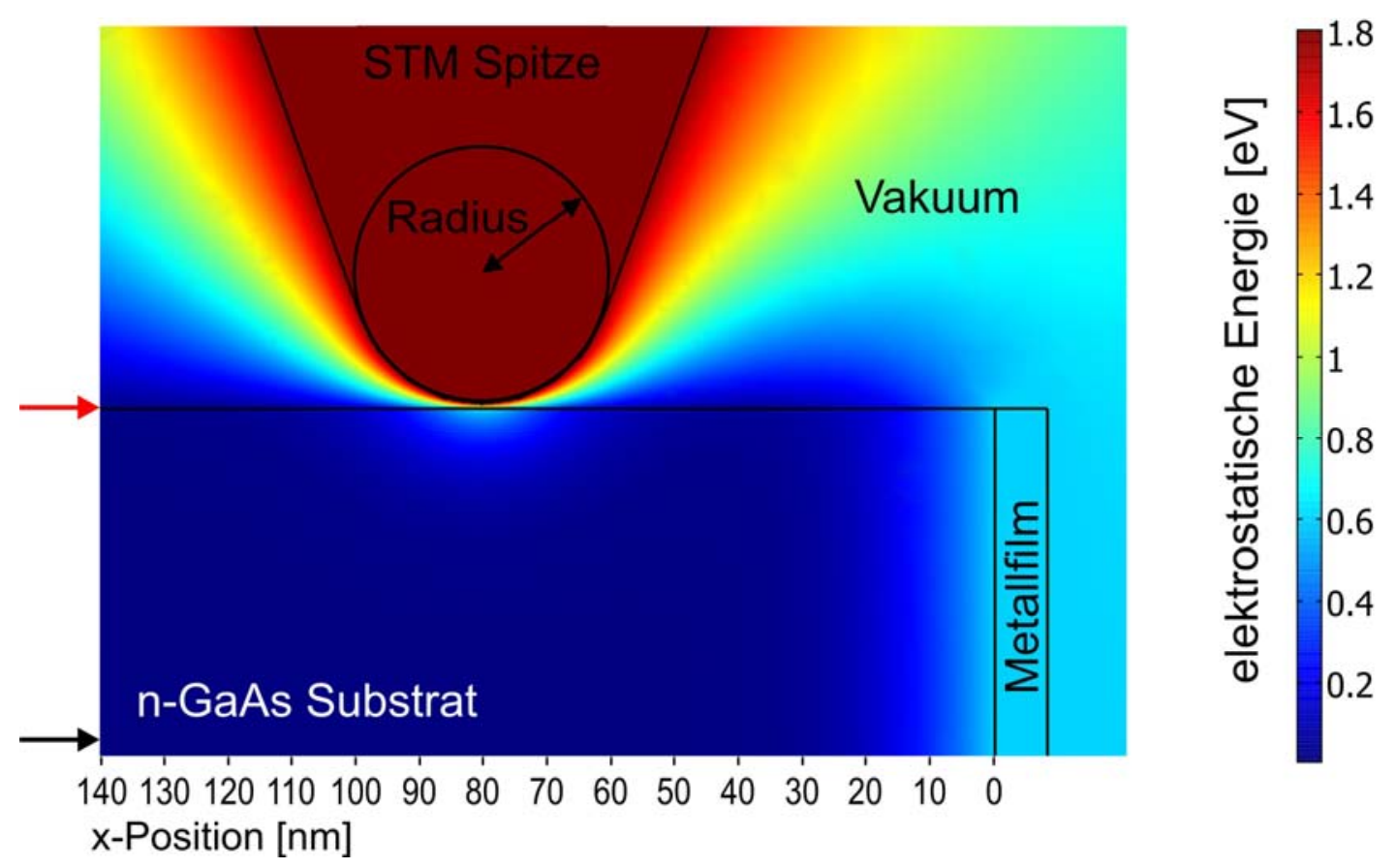

Abbildung 48: Berechneter Verlauf der elektrostatischen Energie (farbkodiert) in einer Ebene senkrecht zum Metallfilm. Die STM Spitze befindet sich in einem Abstand von $80 \mathrm{~nm}$ zum Metallfilm und wird zentral geschnitten. Im Halbleitersubstrat sind die Raumladungszonen unter der STM Spitze und am Metall-Halbleiter Kontakt gut zu erkennen.

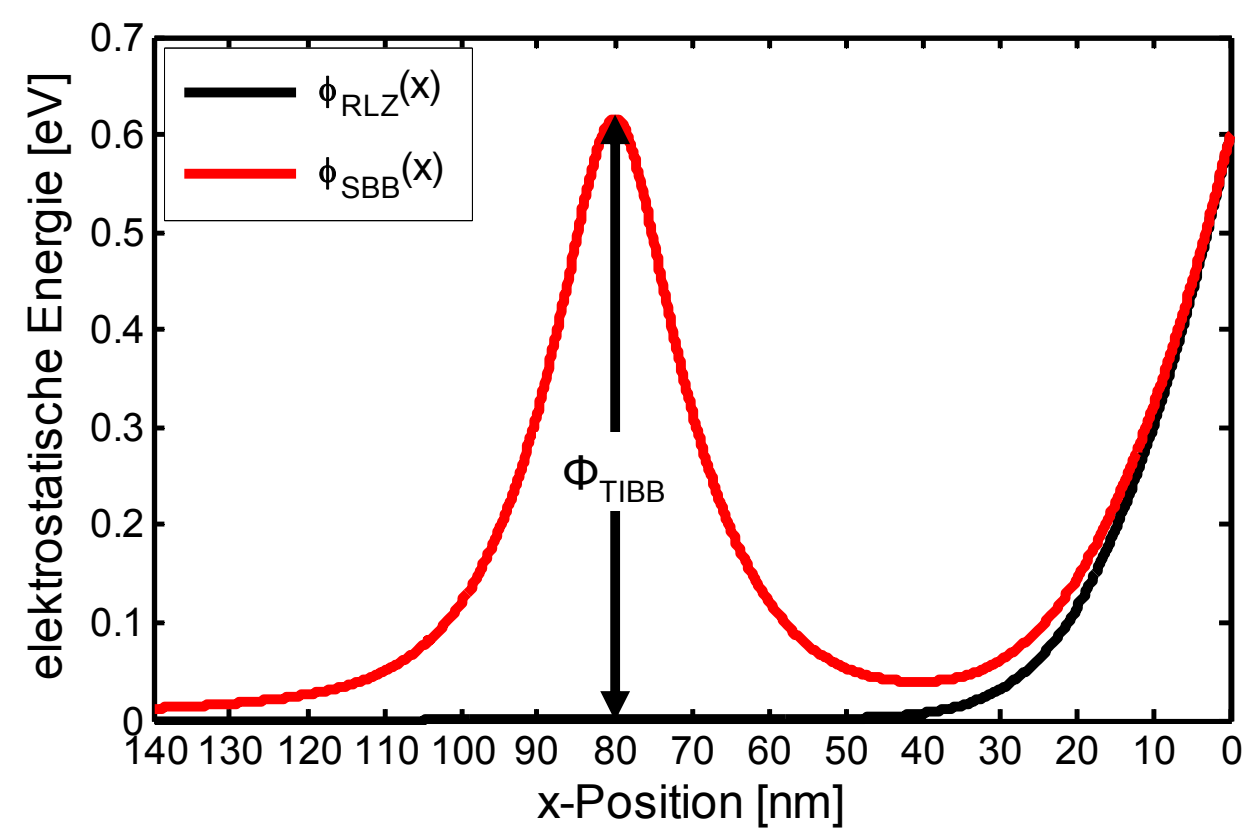

Abbildung 49: Verlauf der elektrostatischen Energie in Höhe der in Abbildung 48 durch die Pfeile markierten Positionen im Bereich des n-GaAs Substrats. Die rote Kurve zeigt den Verlauf direkt an der Oberfläche des Substrats. Im Vergleich dazu zeigt die schwarze Kurve den Verlauf im Volumen, der nicht durch die STM Spitze beeinflusst wird. Das TIBB entspricht der Differenz der beiden Kurven an der x-Position der Spitze. 
Die elektrostatische Energie $\phi_{T S}$ der Spitze relativ zum Halbleitersubstrat beträgt 1,8 eV, die elektrostatische Energie $\phi_{F S}$ des Metallfilms beträgt bei dieser Simulation 0,6 eV. Die Ladungsdichte $\rho(\vec{r})$ wurde für ein n-GaAs Substrat $\left(8 \cdot 10^{17} 1 / \mathrm{cm}^{3}\right.$ Si dotiert) bei $293 \mathrm{~K}$ berechnet. Den Definitionen von $\phi_{T S}$ und $\phi_{F S}$ entsprechend gibt die berechnete elektrostatische Energie, die in Abbildung 48 farbkodiert dargestellt ist, den räumlichen Verlauf des Vakuumniveaus im Berechnungsgebiet an. Im Bereich des GaAs Substrats entspricht dies dem Verlauf der Bandkanten (Rigid-Band-Modell). Sowohl die Raumladungszone des Schottky-Kontakts als auch die spitzeninduzierte Raumladungszone sind deutlich zu erkennen. Nur etwa zwei Drittel des zwischen Spitze und Probe angelegten Potentials fällt über der Vakuumbarriere ab. Der verbleibende Teil wird durch die ionisierten Donatoren in der spitzeninduzierten Raumladungszone abgeschirmt. Abbildung 49 zeigt die elektrostatische Energie im n-GaAs Bereich entlang von Schnitten in Höhe der zwei Pfeile in Abbildung 48. Der Verlauf weit unter der Probenoberfläche, d.h. ohne den Einfluss der STM Spitze, entspricht dem selbstkonsistent berechneten $\phi_{R L Z}(x)$ des Schottky-Kontakts bei $293 \mathrm{~K}$ (schwarze Kurve). Im Volumen des Substrats befindet sich das Vakuum-Niveau den Randbedingungen entsprechend bei $0 \mathrm{eV}$. In einem Abstand von etwa $50 \mathrm{~nm}$ zum Metallfilm beginnt die RLZ des Schottky-Kontakts, in der die elektrostatische Energie bis zum Betrag von $\phi_{F}$ an der Metall-Halbleiter Grenzfläche ansteigt. Die rote Kurve zeigt die Bandverbiegung an der Oberfläche des Substrats (engl. surface band bending, SBB) und wird als $\phi_{S B B}(x)$ bezeichnet. $\phi_{S B B}\left(x_{S p i z z}\right)$ gibt die Bandverbiegung an der x-Position des Spitzenapex an. Den Ausführungen in Kapitel 3.3.1 entsprechend kann $\phi_{\text {TIBB }}$ als Differenz von $\phi_{S B B}\left(x_{\text {Spitze }}\right)$ und $\phi_{R L Z}\left(x_{\text {Spitze }}\right)$ berechnet werden.

Um sowohl den Einfluss des räumlich ansteigenden $\phi_{R L Z}(x)$ als auch die Auswirkungen des Metallfilms auf das TIBB untersuchen zu können, wurden Simulationen für verschiedene Abstände zwischen der STM Spitze und dem Metallfilm durchgeführt. Zur systematischen Variation der Spitzenposition wurde ein Matlab-Skript verwendet, über das der FEM Solver angesprochen werden kann. Um den Einfluss der verschiedenen Parameter der Simulation auf den Verlauf des TIBB in der RLZ zu verdeutlichen, wurde in dem in Abbildung 50 gezeigten Beispiel eine STM Spitze mit einem sehr großen Krümmungsradius von $40 \mathrm{~nm}$ angenommen. Die übrigen Parameter der Simulation sind: Öffnungswinkel der STM Spitze: $30 \mathrm{Grad}$, Tunnelabstand: 0,8 nm, $\phi_{T S}: 1,6 \mathrm{eV}, \phi_{F S}: 0,8 \mathrm{eV}, \rho(\vec{r})$ wurde für ein $\mathrm{n}-\mathrm{GaAs}$ Substrat $\left(8 \cdot 10^{17} \mathrm{1} / \mathrm{cm}^{3}\right.$ Si dotiert $)$ bei $293 \mathrm{~K}$ berechnet. 
(a)

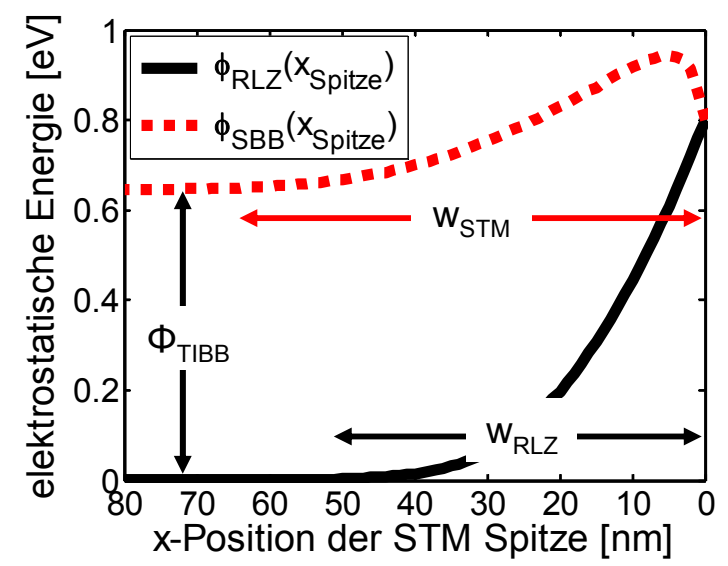

(b)

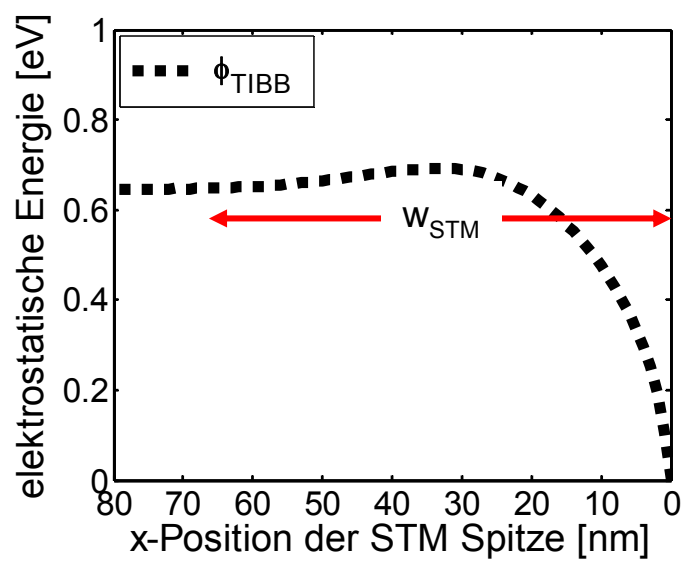

Abbildung 50: (a) Berechnung von $\phi_{S B B}\left(x_{\text {Spitze }}\right)$ direkt unter dem Apex der STM Spitze für unterschiedliche Abstände zwischen Spitze und Metall-Halbleiter Grenzfläche. $\phi_{S B B}\left(x_{\text {Spitze }}\right)$ wird bereits in dem Abstand $w_{S T M}$, der deutlich größer als die Ausdehnung der Raumladungszone $w_{R L Z}$ ist, durch das elektrostatische Potential der Raumladungszone beeinflusst. (b) Berechnung von $\phi_{\text {TIBB }}$ in Abhängigkeit von der x-Position der STM Spitze. Im Abstand $w_{\text {STM }}$ beginnt $\phi_{\text {TIBB }}$ zunächst leicht anzuwachsen, bevor das TIBB aufgrund der hohen Ladungsdichte im Metallfilm auf Null zurückgeht.

Abbildung 50 (a) zeigt $\phi_{S B B}\left(x_{\text {Spitze }}\right)$ in Abhängigkeit von dem Abstand zwischen dem Apex der STM Spitze und der Metall-Halbleiter Grenzfläche. Der Einfluss der Raumladungszone und des Metallfilms ist deutlich zu erkennen. Aufgrund der lateralen Ausdehnung der spitzeninduzierten Raumladungszone (siehe Abbildung 49, rote Kurve) wird $\phi_{S B B}\left(x_{S p i t z e}\right)$ bereits in dem Abstand $w_{S T M}$, d.h. ohne dass sich der Apex der STM Spitze im Bereich der Raumladungszone mit der Ausdehnung $w_{R L Z}$ befindet, signifikant beeinflusst. Wie in Abbildung 50 (b) zu sehen ist, steigt $\phi_{\text {TIBB }}$ als Differenz zwischen $\phi_{S B B}\left(X_{\text {Spitze }}\right)$ und $\phi_{R L Z}\left(X_{\text {Spizze }}\right)$ mit geringer werdendem Abstand zunächst an. Auf der anderen Seite wird die Abschirmung des Potentials zwischen Spitze und Probe umso effektiver, je näher sich die Spitze an dem Metallfilm mit seiner hohen Ladungsdichte befindet. $\phi_{\text {ТІвB }}$ wird erwartungsgemäß wieder geringer und geht im Bereich der Metall-Halbleiter Grenzfläche nahezu auf Null zurück. Eine Zusammenstellung der Auswirkungen, die die verschiedenen freien Parameter der Simulation auf den Verlauf von $\phi_{\text {TIBB }}$ in der RLZ haben, findet sich in Anhang A.

Die Simulationen zeigen, dass es durch die Verwendung der Finite Elemente Methode gelungen ist, neben der dreidimensionalen Spitzengeometrie auch den Einfluss des Metallfilms in die selbstkonsistente Berechnung der elektrostatischen Potentiallandschaft einzubeziehen. Insbesondere wird deut- 
lich, wie stark das TIBB in der RLZ durch den angrenzenden Metallfilm modifiziert wird. Auf den konkreten Einfluss von $\phi_{\text {ТІвB }}$ auf die I(U)-Spektroskopie in der RLZ wird detailliert in Kapitel 7.1.3 eingegangen.

\subsubsection{Vergleich zwischen der Berechnung des TIBB durch die Finite Ele- mente Methode mit dem Ansatz von Feenstra}

Bei der FEM Simulation handelt es sich um eine näherungsweise numerische Berechnung der 3DPotentiallandschaft, deren Güte stark von der Diskretisierung der finiten Elemente abhängig ist. Um die Eignung dieser Methode zur Berechnung des TIBB zu überprüfen, zeigt Abbildung 51 einen Vergleich mit der allgemein anerkannten 3D-Simulation des TIBB nach Feenstra für den Fall eines homogenen n-GaAs Substrats [80, 95, 97].

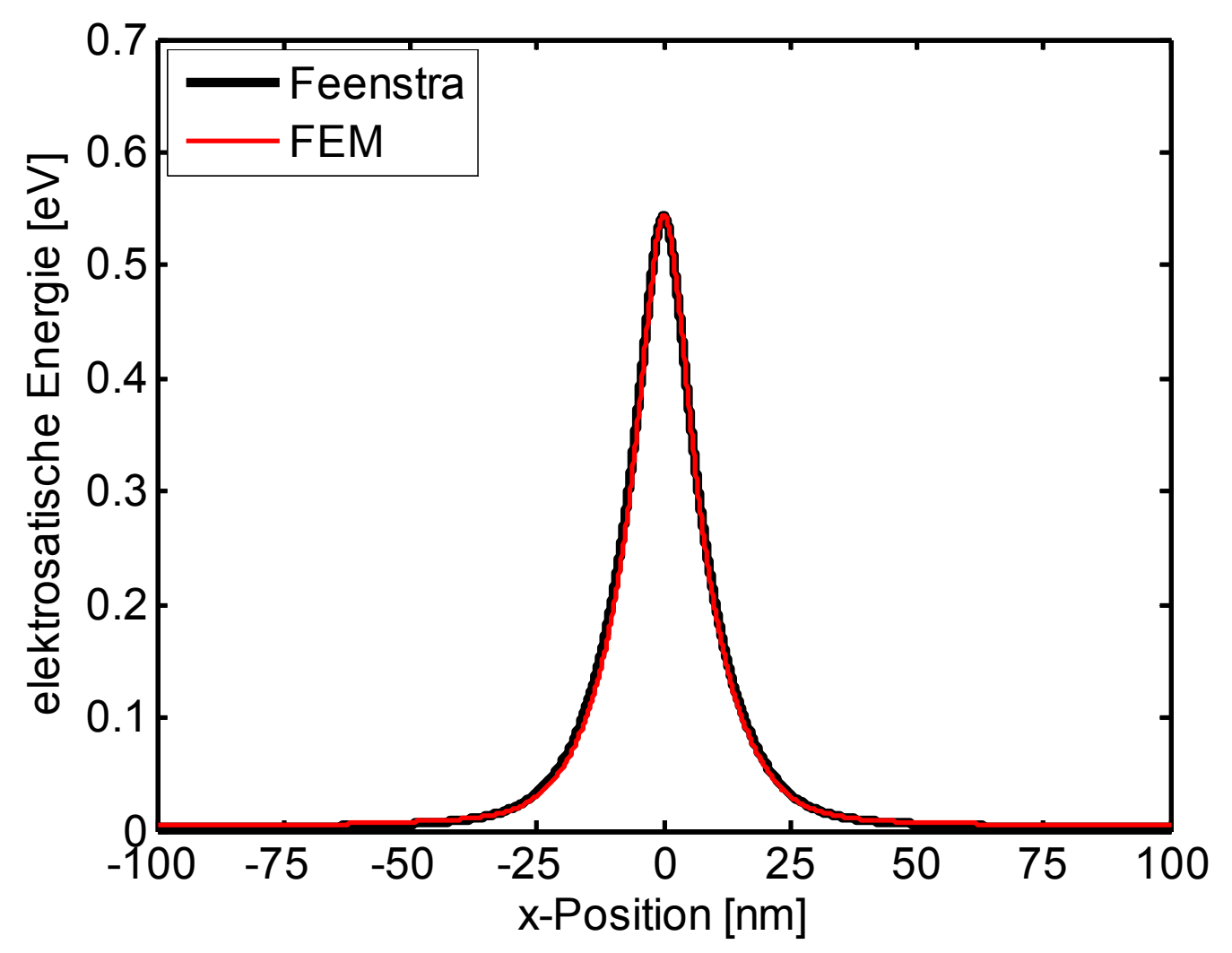

Abbildung 51: Vergleich des mit der Finite Elemente Methode berechneten Verlaufs der elektrostatischen Energie $\phi_{S B B}(x)$ an der Oberfläche eines homogenen $n$-GaAs Substrats mit der Lösung nach Feenstra [97].

Abbildung 51 stellt die mit den beiden Methoden berechneten Verläufe der elektrostatischen Energie $\phi_{S B B}(x)$ gegenüber. Für die Berechnung wurden jeweils dieselben Parameter gewählt (Spitzenradius: 10 nm, Öffnungswinkel: 60,9 Grad, Tunnelabstand: $8 \AA, \phi_{T S}: 2 \mathrm{eV}$, Si-Konzentration: $8 \cdot 10^{17} 1 / \mathrm{cm}^{3}$, 
Temperatur: $6 \mathrm{~K}){ }^{23}$ Beide Methoden liefern sowohl qualitativ als auch quantitativ nahezu identische Ergebnisse. Der Betrag von $\phi_{T I B B}$, d.h. $\phi_{S B B}\left(x_{\text {Spitze }}\right)$, unterscheidet sich um lediglich 3 meV. Die Varianz in dem gesamten in Abbildung 51 dargestellten Bereich beträgt $1,48 \cdot 10^{-5} \mathrm{meV}^{2}$. Die exzellente Übereinstimmung zeigt, dass der Einsatz der Finite Elemente Methode in der beschriebenen Art und Weise eine verlässliche Berechnung des TIBB erlaubt.

\subsubsection{Die Oberflächenbandverbiegung bei positiven Bias-Spannungen als Verbindung zwischen Simulation und Experiment}

Gemäß Relation (2-7) können bei einer STM Messung Zustände in dem durch die Bias-Spannung vorgegebenen Energieintervall zwischen der Fermi-Energie der Spitze und der Fermi-Energie der Probe zu dem Tunnelstrom beitragen. Im Fall einer halbleitenden Probe verringert sich das Energieintervall bei positiven Bias-Spannungen um $E_{C}-\mu_{c}$, da in dem Bereich der Volumenbandlücke keine Tunnelprozesse stattfinden können. Bei der hier untersuchten n-GaAs(110) Spaltfläche muss darüber hinaus beachtet werden, dass der Verlauf der Bandkanten durch das TIBB und gegebenenfalls auch durch das elektrostatische Potential in der RLZ modifiziert wird. Abbildung 52 zeigt in diesem Zusammenhang schematische 1D-Bandmodelle aus dem Bereich des Tunnelkontakts, in denen sowohl die Situation ohne als auch mit einem $\phi_{R L Z}$ bei einer festen positiven Bias-Spannung skizziert wird.

\footnotetext{
${ }^{23}$ Die Spitzengeometrien stimmen nicht exakt überein, da in [97] eine hyperbolische Spitzenform verwendet wird, die nur näherungsweise durch die Parameter Krümmungsradius und Öffnungswinkel beschrieben werden kann.
} 

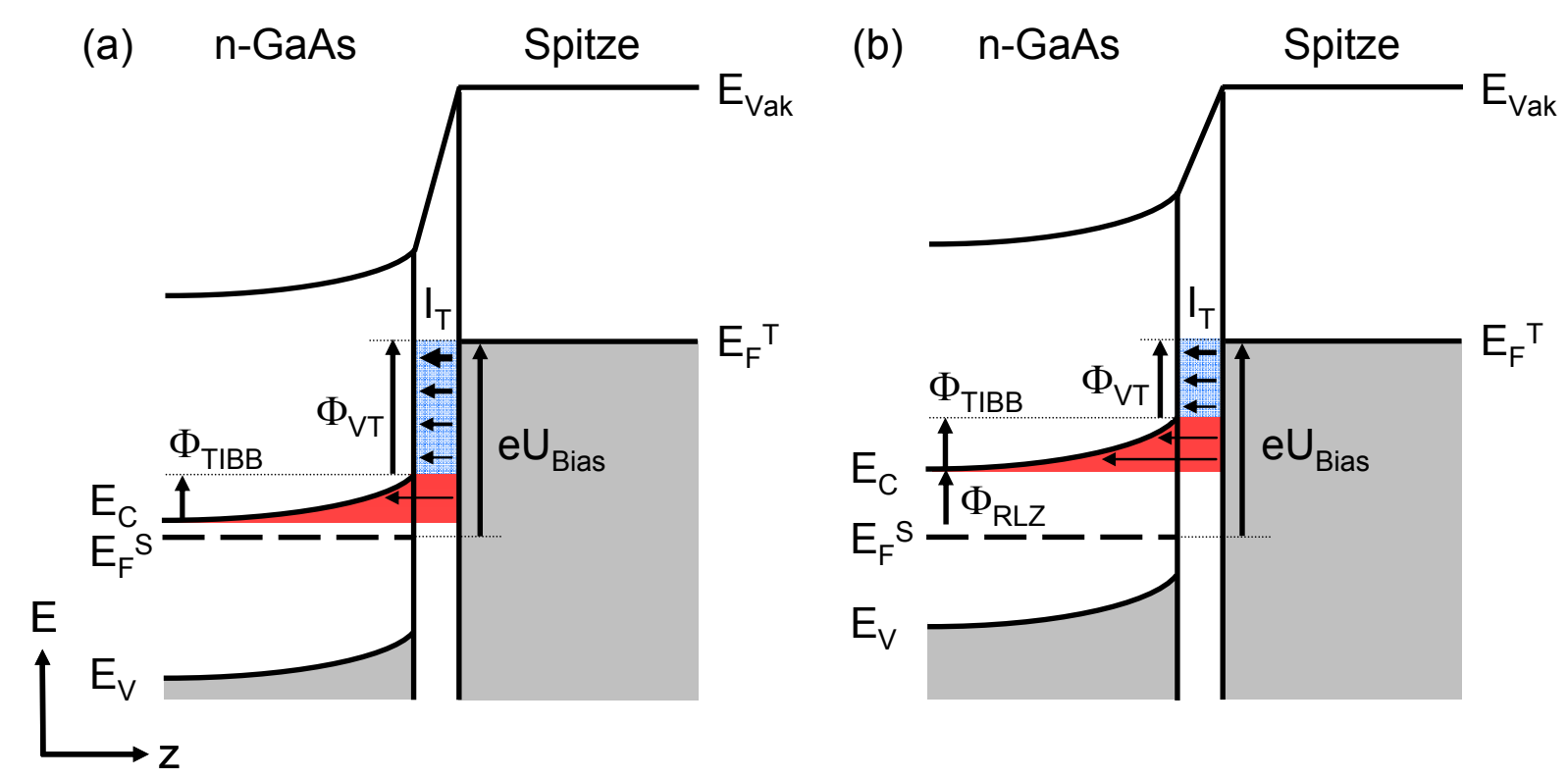

Abbildung 52: Schematische 1D-Bandmodelle des Tunnelexperiments bei einer festen positiven Bias-Spannung. (a) Außerhalb der RLZ des Schottky-Kontakts bestimmt das TIBB die Oberflächenbandverbiegung. Neben dem blau skizzierten Energiebereich, in dem ausschließlich die Vakuumbarriere durchtunnelt werden muss, können auch in dem rot dargestellten Energieintervall Tunnelprozesse stattfinden. In diesem Fall muss zusätzlich die spitzeninduzierte Raumladungszone durchtunnelt werden. (b) In der RLZ des Schottky-Kontakts wird die Oberflächenbandverbiegung zusätzlich durch $\phi_{R L Z}$ bestimmt.

Das Energieintervall, in dem es zu Tunnelprozessen kommen kann, kann in zwei Bereiche unterteilt werden:

- Bereich 1: Zwischen der Fermi-Energie der Spitze und der Leitungsbandkante an der Oberfläche des Substrats kann der Tunnelstrom in dem Modell nach Hamers (vergleiche Kapitel 2.2) durch (2-7) und (2-8) beschrieben werden, da ausschließlich die Vakuumbarriere durchtunnelt wird (blau hinterlegter Bereich in Abbildung 52). Im Folgenden wird dieses Energieintervall mit $\phi_{V T}$ bezeichnet.

$$
\phi_{V T}=e U_{\text {Bias }}-\phi_{T I B B}-\phi_{R L Z}-\left(E_{C}-\mu_{c}\right)
$$

- Bereich 2: Zwischen der energetischen Position der Leitungsbandkante an der Oberfläche des Substrats und der Volumenposition muss zusätzlich zur Vakuumbarriere die spitzeninduzierte Raumladungszone durchtunnelt werden (rot hinterlegter Bereich in Abbildung 52). Die Ausdehnung des Energieintervalls entspricht $\phi_{\text {TIBB }}$. 
Abbildung 52 (b) zeigt, dass zum einen das Gesamtintervall, in dem es zu Tunnelprozessen kommen kann, durch $\phi_{R L Z}$ verringert wird. Zum anderen verändert sich aber auch der relative Anteil, den Zustände in den beiden Teilbereichen zu dem Gesamttunnelstrom beitragen, da $\phi_{\text {TIBB }}$ im Gegensatz zu $\phi_{V T}$ in der RLZ des Schottky-Kontakts mit geringer werdendem Abstand zum Metallfilm zunächst zunimmt (siehe Kapitel 7.1.2.5). Um quantitativ berechnen zu können, wie sich derartige Veränderungen der Tunnelbedingungen auf die I(U)-Spektroskopie auswirken, kann die IVCHAR Simulation mit der im vorangegangenen Kapitel beschriebenen FEM Simulation verknüpft werden. Bei diesem Verfahren muss zunächst der Potentialverlauf im Bereich von Vakuumbarriere und Halbleitersubstrat unter Berücksichtigung der experimentellen Gegebenheiten mit der Finite Elemente Methode simuliert werden. Für diesen Potentialverlauf kann der Tunnelstrom in einem zweiten Schritt mit IVCHAR energieabhängig berechnen werden [91].

Im Fall der in Kapitel 7.1 präsentierten XSTS Messung kann auf diesem Weg unter anderem nachvollzogen werden, bis zu welchem Umfang Tunnelprozesse durch die spitzeninduzierte RLZ zu dem Tunnelstrom der Spektren beigetragen haben. Um dies zu erreichen, muss zunächst bestimmt werden, in welcher Konstellation das maximale $\phi_{\text {TIBB }}$ aufgetreten ist. Abbildung 53 zeigt dazu den mit der Finite Elemente Methode berechneten Verlauf von $\phi_{\text {TIBB }}$ in der RLZ bei der maximal angelegten positiven Bias-Spannung von $2 \mathrm{~V}$.

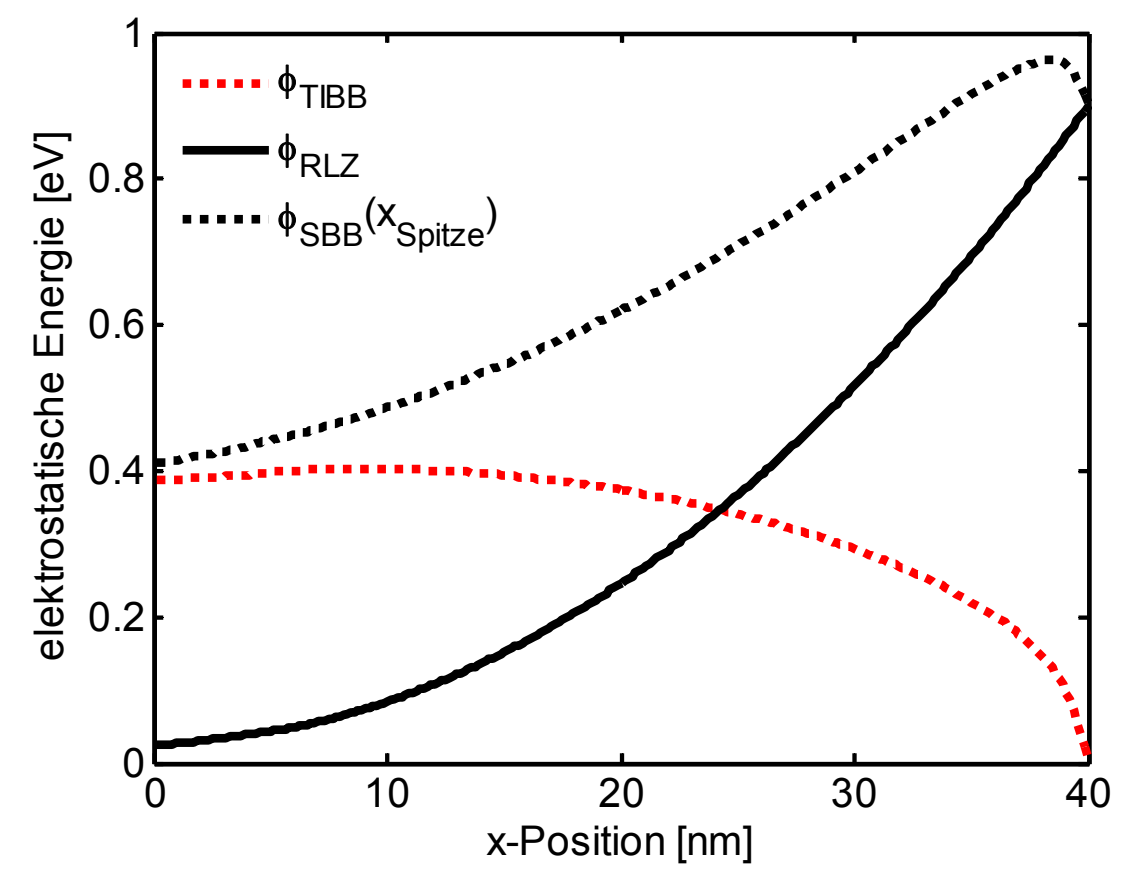

Abbildung 53: Simulierter Verlauf von $\phi_{\text {TIBB }}$ im Bereich der RLZ (Details im Text). Die MetallHalbleiter Grenzfläche befindet sich in der Simulation bei $40 \mathrm{~nm}$. Die Verläufe von $\phi_{R L Z}$ und $\phi_{S B B}\left(X_{\text {Spitze }}\right)$ sind ebenfalls dargestellt. 
Bei der SBH des simulierten Schottky-Kontakts wurde in Anlehnung an die Abschätzung in Kapitel 6.3 ein Wert von 0,9 eV angenommen. Im Fall der STM Spitze wurde der experimentell bestimmte Krümmungsradius von $3 \mathrm{~nm}$ (siehe Kapitel 6.2), ein Öffnungswinkel von 40 Grad (siehe Kapitel 2.6) und eine Spitzenaustrittsarbeit $\phi_{T}=4 \mathrm{eV}$ vorgegeben [80, 95]. Bei dem Tunnelabstand $d$ wurde ein typischer Wert von $8 \AA$ angenommen [72, 93, 100]. Die Ladungsdichte im Halbleitersubstrat wurde für eine Si-Dotierung von $8 \cdot 10^{17} 1 / \mathrm{cm}^{3}$ (Herstellerangabe) bei $293 \mathrm{~K}$ berechnet.

Aus der Simulation geht hervor, dass bei der in Kapitel 7.1 gezeigten XSTS Messung das maximale $\phi_{\text {тівв }}$ von etwa 0,4 eV in einem Abstand von $30 \mathrm{~nm}$ zur Metall-Halbleiter Grenzfläche aufgetreten ist. Der Tunnelstrom, der unter diesen Bedingungen geflossen ist, kann anschließend mit IVCHAR berechnet werden, indem die Simulation sowohl an die FEM Simulation als auch an das Experiment angepasst wird:

- Die freien Parameter der IVCHAR Simulation werden so gewählt, dass der selbstkonsistent berechnete Potentialverlauf bei einer Bias-Spannung von 2 V dem Ergebnis der FEM Simulation sowohl im Bereich der Vakuumbarriere als auch in Bezug auf die relevanten Größen $\phi_{\text {TIBB }}$ und $\phi_{R L Z}$ entspricht.

- Über die Fläche $A_{T}$ der Spitze, durch die der Tunnelstrom fließt, wird der berechnete Tunnelstrom an den experimentell bestimmten Tunnelstrom von 85 pA angepasst, der in einem Abstand von $30 \mathrm{~nm}$ zum Metallfilm bei einer Bias-Spannung von $2 \mathrm{~V}$ gemessen wurde. ${ }^{24}$

Durch die beschriebene Verknüpfung der FEM Simulation mit der IVCHAR Simulation kann gezeigt werden, dass Tunnelprozesse durch die spitzeninduzierte RLZ in der berechneten Situation lediglich 2,9 $\cdot 10^{-3}$ pA zu dem Gesamttunnelstrom beigetragen haben. Es handelt sich demnach um einen Beitrag, der deutlich unter der experimentellen Auflösungsgrenze von etwa 1 pA liegt. Da die Simulation bereits die Konstellation beschreibt, in der im Fall der in Kapitel 7.1 präsentierten STS Messung das maximale $\phi_{\text {TIBB }}$ aufgetreten ist, kann dieser Anteil bei der Analyse der I(U)-Kennlinien generell vernachlässigt werden.

Der bei positiven Bias-Spannungen auftretende Tunnelstrom hängt demnach unmittelbar mit dem Energieintervall $\phi_{V T}$ zwischen der Fermi-Energie der Spitze und der energetischen Lage der Leitungsbandkante an der Oberfläche des Substrats zusammen (blau hinterlegter Bereich in Abbildung 52). Da dieser Zusammenhang unabhängig von dem Abstand zum Metallfilm gilt, entsprechen die positiven Bias-Spannungen, bei denen im Fall des experimentellen Spektrenschnitts in Abbildung 43 (b) jeweils

${ }^{24}$ Zur Bestimmung des Tunnelstroms werden die normierten I(U)-Kennlinien verwendet. 
derselbe Tunnelstrom $I_{T, R E F}$ gemessen wurde, auch den Bias-Spannungen, bei denen jeweils dasselbe Energieintervall $\phi_{V T, R E F}$ vorgelegen hat:

$$
U_{\text {Bias }}\left(x, I_{T, R E F}\right) \cong U_{\text {Bias }}\left(x, \phi_{V T, R E F}\right)
$$

Diese Äquivalenz erlaubt es, eine direkte Verbindung zwischen den experimentellen Daten und den FEM Simulationen herzustellen, ohne dass eine konkrete Berechnung des Tunnelstroms erforderlich ist. Das Energieintervall $\phi_{V T}$ spielt daher bei der in den folgenden Abschnitten diskutierten Analyse der XSTS Daten eine zentrale Rolle.

\subsubsection{Auswirkungen des TIBB auf die I(U)-Spektroskopie in der Raum- ladungszone des Schottky-Kontakts}

Um Veränderungen in den in Kapitel 7.1 gezeigten experimentellen I(U)-Kennlinien identifizieren zu können, die durch das ansteigende $\phi_{R L Z}$ in Verbindung mit dem lokal variierenden $\phi_{\text {TIBB }}$ verursacht werden, ist es gemäß Relation (7-9) ausreichend, $\phi_{V T}$ in Abhängigkeit von der Bias-Spannung und der Position in der RLZ des Schottky-Kontakts im Rahmen der FEM Simulation zu berechnen. Um dies zu verdeutlichen, zeigt Abbildung 54 (a) zunächst den relevanten Teil des normierten Spektrenschnitts aus Abbildung 43 (b), der bei positiven Bias-Spannungen im Bereich des GaAs Substrats aufgenommen wurde. Der dargestellte Ausschnitt endet 1,1 nm vor der Metall-Halbleiter Grenzfläche, da es bei geringeren Abständen durch das in Kapitel 6.1 beschriebene Spitzenartefakt bereits zu Tunnelprozessen in den Fe-Film gekommen ist. Die in die I(U)-Kennlinien eingezeichnete Konturlinie $U_{\text {Bias }}\left(x, I_{T}=10 p A\right)$ gibt an, bei welchen Bias-Spannungen jeweils ein Tunnelstrom von 10 pA gemessen wurde. Gemäß Relation (7-9) entspricht diese Konturlinie im Rahmen der experimentellen Auflösung dem Verlauf von $U_{B i a s}\left(x, \phi_{V T, R E F}\right)$, wobei der Wert von $\phi_{V T, R E F}$, der unter anderem von der Spitzenaustrittsarbeit $\phi_{T}$ abhängt, selbst nicht bekannt ist. 

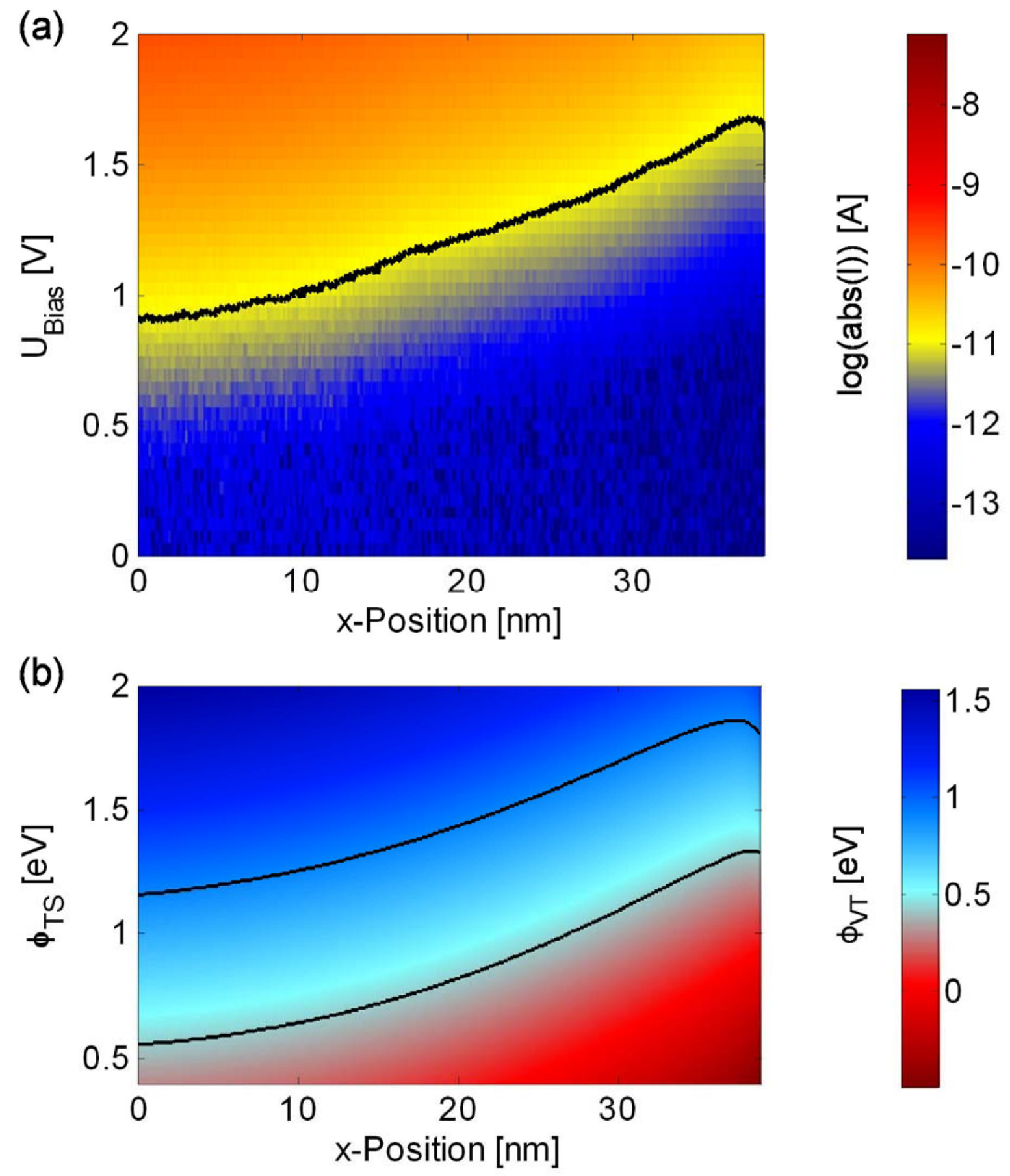

Abbildung 54: (a) Ausschnitt des Spektrenschnitts aus Abbildung 43 (b), der im Bereich des GaAs Substrats aufgenommen wurde. Die Darstellung ist auf den Teil der I(U)-Kennlinien beschränkt, der bei positiven Bias-Spannungen aufgezeichnet wurde. Die schwarze Linie markiert die BiasSpannungen, bei denen jeweils ein Referenzstrom von 10 pA gemessen wurde. (b) FEM Simulation von $\phi_{V T}$ (farbkodiert) als Funktion von $\phi_{T S}$ in Abhängigkeit von dem Abstand zum Metallfilm. Die Parameter der Simulation sind identisch mit denen, die zur Simulation der in Abbildung 53 gezeigten Daten verwendet wurden. Die eingezeichneten Konturlinien entsprechen dem Verlauf von zwei unterschiedlich großen $\phi_{V T}$. 
Abbildung 54 (b) stellt den experimentellen Daten die FEM Simulation von $\phi_{V T}$ in Abhängigkeit von $\phi_{T S}$ gegenüber. Gemäß Relation (7-5) entspricht $\phi_{T S}$ bis auf einen konstanten Betrag, der sich aus der Differenz der Austrittsarbeiten von Spitze und Probe ergibt, $e \cdot U_{\text {Bias }}$. Da der Wert von $\phi_{V T, R E F}$ aus den experimentellen Daten nicht hervorgeht, geben die eingezeichneten Konturlinien exemplarisch die Verläufe $\phi_{T S}\left(x, \phi_{V T}=\right.$ konst. $)$ von zwei unterschiedlich großen Energieintervallen $\phi_{V T}$ wieder. Sie zeigen, dass im Bereich des GaAs Substrats in Abhängigkeit von der Größe des Energieintervalls $\phi_{V T}$ eine sehr gute qualitative Übereinstimmung zwischen dem Verlauf von $U_{B i a s}\left(x, I_{T}=10 p A\right)$ und den simulierten Verläufen von $\phi_{T S}\left(x, \phi_{V T}=\right.$ konst.) existiert. Im Gegensatz zu den in Kapitel 7.1.1 gezeigten IVCHAR Simulationen gilt dies auch für den an den Metallfilm angrenzenden Bereich, in dem die experimentell beobachtete Verschiebung des Einsatzpunktes zu geringeren Bias-Spannungen durch die FEM Simulationen reproduziert wird.

Aus der generellen Übereinstimmung kann geschlossen werden, dass die charakteristischen Veränderungen der I(U)-Kennlinien in der RLZ eines Schottky-Kontakts im Wesentlichen durch das ansteigende $\phi_{R L Z}$ in Verbindung mit dem lokal variierenden $\phi_{T I B B}$ hervorgerufen werden. Im folgenden Kapitel wird gezeigt, dass tatsächlich alleine die Variation der SBH des simulierten Systems ausreicht, um bei einem definierten $\phi_{V T}$ eine quantitative Übereinstimmung mit dem experimentell bestimmten Verlauf zu erzielen.

\subsection{Bestimmung der Schottky-Barrierenhöhe}

Eine zentrale Größe zur Beschreibung der elektronischen Eigenschaften eines Schottky-Kontakts ist seine charakteristische Schottky-Barrierenhöhe. Da es sich um eine Größe handelt, die unmittelbar mit dem Phänomen des FLP verknüpft ist, bauen die meisten experimentellen Arbeiten zur Untersuchung der Schottky-Barrierenformierung auf der Messung von SBHs auf. Sie wird üblicherweise durch Strom-Spannungs-Kennlinien (I-V) bzw. Kapazitäts-Spannungs-Kennlinen (C-V) oder Methoden der Photo-Emissions-Spektroskopie (PES) in Form einer makroskopischen mittleren SBH bestimmt [2, 20, 55]. Die Einführung von lokal aufgelösten Methoden wie der Ballistischen-ElektronenEmissions-Mikroskopie (BEEM) hat allerdings gezeigt, dass es sich hierbei in der Regel um eine effektive SBH handelt, die durch laterale Inhomogenitäten auf der Nanometerskala beeinflusst wird [21-23, 136]. Die quantitative Bestimmung der SBH eines Systems ist daher eine wichtige Vorraussetzung, um die Relevanz der in Kapitel 1 diskutierten Modelle experimentell überprüfen zu können.

Im weiteren Verlauf des Kapitels wird gezeigt, dass die XSTS Untersuchung eines Schottky-Kontakts eine ideale Messmethode darstellt, um nicht nur die SBH mit hoher Genauigkeit bestimmen zu kön- 
nen. Sie bietet darüber hinaus die Möglichkeit, auch den Einfluss lokaler Defekte auf den Potentialverlauf in der RLZ zu untersuchen, was mit den bislang verfügbaren experimentellen Methoden nicht erreicht werden kann.

In Kapitel 7.1 wurde durch verschiedene Simulationen gezeigt, dass das elektrostatische Potential in der RLZ des Fe-GaAs(110) Schottky-Kontakts in Verbindung mit dem variierenden TIBB zu charakteristischen Veränderungen in der I(U)-Spektroskopie führt. Da diese Modifikationen nicht nur von dem Verlauf von $\phi_{R L Z}$ abhängen, sondern darüber hinaus von allen Parametern, durch die das TIBB beeinflusst wird, kann die SBH des Schottky-Kontakts nicht direkt aus den experimentellen Daten bestimmt werden. Durch den in Kapitel 7.1.3 geschilderten Zusammenhang zwischen dem experimentellen Verlauf von $U_{\text {Bias }}\left(x, I_{T, R E F}\right)$ und der FEM Simulation ist es allerdings möglich, die Auswirkungen der verschiedenen Parameter auf die I(U)-Kennlinien zusammenhängend zu berechnen, so dass die SBH über den Vergleich zwischen Simulation und Experiment bestimmt werden kann. Dabei kann ausgenutzt werden, dass einige Parameter der Messanordnung mit hoher Genauigkeit experimentell zugänglich sind. Dazu zählen sowohl die Position der Metall-Halbleiter Grenzfläche (Kapitel 6.4) als auch der Krümmungsradius und ggf. der Öffnungswinkel der STM Spitze (Kapitel 6.2). Dies ermöglicht es, den Parameterraum der Simulationen auf die Barrierenhöhe $\phi_{S B}$, die Austrittsarbeit der Spitze $\phi_{T}$ und den Tunnelabstand $d$ einzugrenzen.

Da im Rahmen der FEM Simulation von einem homogenen n-GaAs Substrat ausgegangen wird, kann eine Übereinstimmung zwischen Simulation und Experiment prinzipiell nur dann erreicht werden, wenn lokale Defekte keinen Einfluss auf den experimentellen Verlauf von $U_{B i a s}\left(x, I_{T, R E F}\right)$ haben. Um diese Voraussetzung weitestgehend erfüllen zu können, sollte der Verlauf entlang eines Schnittes bestimmt werden, in dessen Nähe sich möglichst wenige Defekte befinden. Da die Position des in Kapitel 7.1 diskutierten Spektrenschnitts bereits nach diesem Kriterium ausgewählt wurde, eignet sich dieser Datensatz ideal, um im Folgenden die Barrierenhöhe des Fe-GaAs(110) Schottky-Kontakts zu bestimmen.

Wie in Kapitel 7.1.4 beschrieben werden dazu zunächst abstandsabhängige FEM Simulation von $\phi_{V T}$ als Funktion von $\phi_{T S}$ für unterschiedliche $\phi_{S B}$ bei einem festen $d$ von $8 \AA$ durchgeführt. Bei dem Krümmungsradius und dem Öffnungswinkel der Spitze werden die experimentell bestimmten Werte von $3 \mathrm{~nm}$ und 40 Grad zugrunde gelegt. Zur Berechnung der Ladungsdichte im GaAs Substrat wird gemäß der Herstellerangabe eine Si-Dotierung von $8 \cdot 10^{17} 1 / \mathrm{cm}^{3}$ bei $293 \mathrm{~K}$ verwendet. In diesen Datensätzen werden die Verläufe $\phi_{T S}\left(x, \phi_{V T}=\right.$ konst. $)$ von unterschiedlich großen Intervallen berechnet und mit dem experimentellen Verlauf verglichen. 


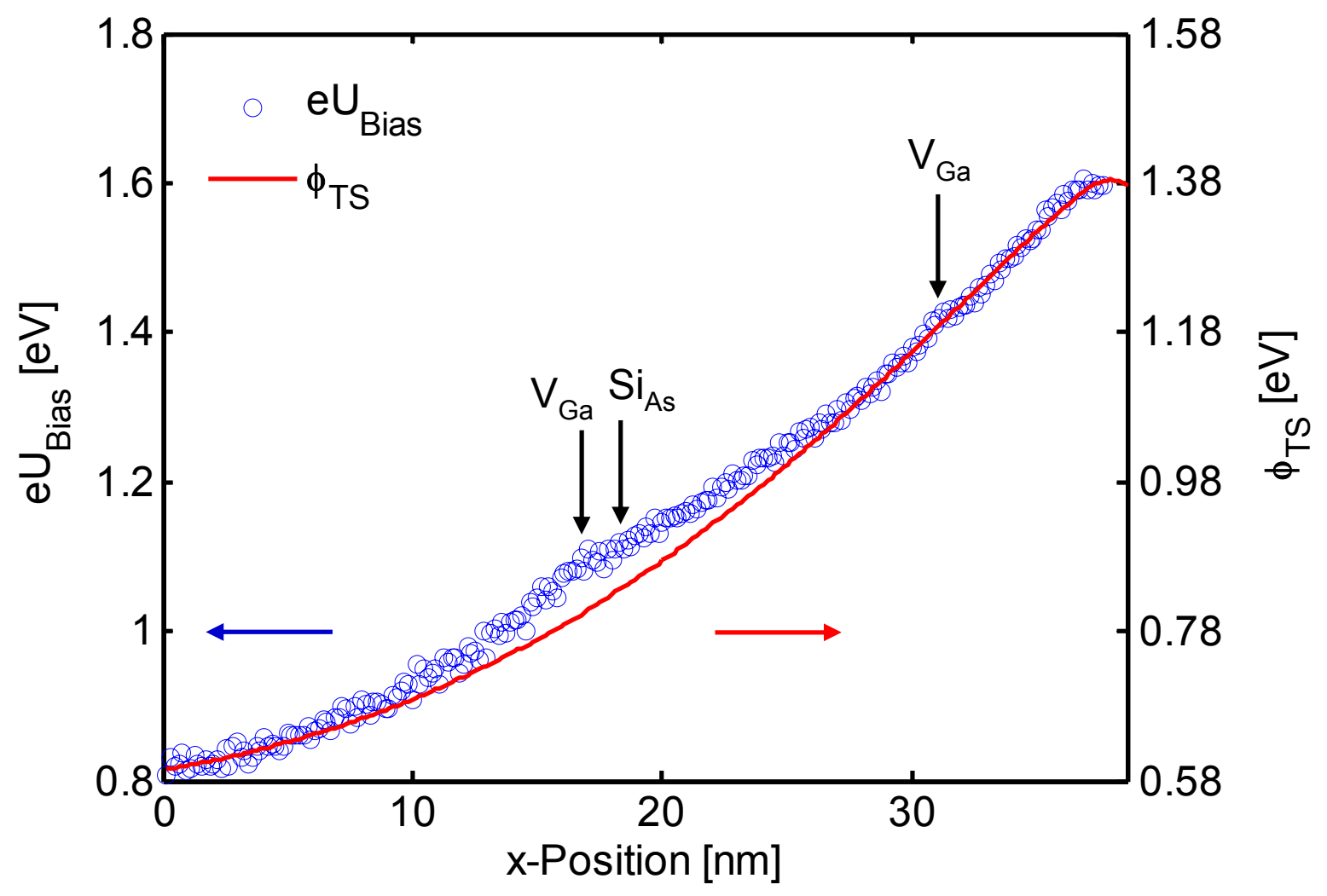

Abbildung 55: Vergleich des experimentell bestimmten Verlaufs von e $U_{\text {Bias }}\left(x, I_{T}=10 p A\right)$ (entspricht der Konturlinie in dem Spektrenschnitt in Abbildung 54 (a)) mit dem berechneten Verlauf von $\phi_{T S}\left(x, \phi_{S B}=0,92 \mathrm{eV}, \phi_{V T}=0,44 \mathrm{eV}, d=8 \mathrm{Ang}\right)$. Die $x$-Positionen der Defekte, die sich in der Nähe des Spektrenschnitts befinden, sind eingezeichnet (siehe Abbildung 43 (a)).

Abbildung 55 zeigt, dass im Fall eines Tunnelabstands von $8 \AA$ eine weitgehende Übereinstimmung mit dem experimentellen Verlauf von $e U_{\text {Bias }}\left(x, I_{T}=10 p A\right)$ bei einer SBH von 0,92 eV und einem Energieintervall $\phi_{V T}$ von 0,44 eV erreicht wird. Die Bereiche, in denen Abweichungen zwischen dem experimentellen und dem berechneten Verlauf verbleiben, können den in Abbildung 43 (a) identifizierten Defekten zugeordnet werden, die sich in der Nähe des Spektrenschnitts befinden. Dem in Kapitel 6.4 beschriebenen Kontrast der Defekte entsprechend ist der Einfluss der Ga-Fehlstellen ( $\mathrm{V}_{\mathrm{Ga}}$ ) lokal sehr begrenzt, da sie in der RLZ des Schottky-Kontakts ungeladen sind. Im Gegensatz dazu beeinflussen Dotieratome wie der $\mathrm{Si}_{\mathrm{As}}$-Akzeptor den Verlauf von $e U_{\text {Bias }}\left(x, I_{T}=10 \mathrm{pA}\right)$ auf einer Längenskala von etwa $10 \mathrm{~nm}[80,137]$.

Anhand der beiden Skalen von Abbildung 55 kann die ansonsten konstante Differenz von 0,22 eV zwischen dem Verlauf von $e U_{\text {Bias }}\left(x, I_{T}=10 p A\right)$ und $\phi_{T S}\left(x, \phi_{S B}=0,92 \mathrm{eV}, \phi_{V T}=0,44 \mathrm{eV}\right)$ bestimmt werden. Sie entspricht gemäß Relation (7-5) dem Unterschied der Austrittsarbeiten von Spitze $\phi_{T}$ und Probe $\phi_{S}$. Eine quantitative Übereinstimmung des berechneten Verlaufs von 
$\phi_{T S}\left(x, \phi_{S B}=0,92 \mathrm{eV}, \phi_{V T}=0,44 \mathrm{eV}, d=8 \mathrm{Ang}\right)$ mit dem experimentellen Verlauf existiert demnach im Fall einer Spitzenaustrittsarbeit von $\phi_{T}=3,85 \mathrm{eV}$.

Um die Abweichung zwischen dem experimentellen Verlauf von $e U_{\text {Bias }}\left(x, I_{T}=10 p A\right)$ und einem berechneten Verlauf von $e U_{B i a s}\left(x, \phi_{S B}, \phi_{V T}, d\right)$ zu quantifizieren, wird im Folgenden in Analogie zur Standardabweichung die Wurzel der mittleren quadratischen Abweichung zwischen dem Messwert von $e U_{\text {Bias }}\left(x_{i}, I_{T}=10 p A\right)$ am Ort $x_{i}$ und dem berechneten Wert von $e U_{B i a s}\left(x_{i}, \phi_{S B}, \phi_{V T}, d\right)$ am Ort $x_{i}$ verwendet:

$$
\sigma=\sqrt{\frac{1}{n-1} \sum_{i=1}^{n}\left(e U_{\text {Bias }}\left(x_{i}, I_{T}=10 p A\right)-e U_{\text {Bias }}\left(x_{i}, \phi_{S B}, \phi_{V T}, d\right)\right)^{2}}
$$

Abbildung 56 macht deutlich, dass die Übereinstimmung zwischen Simulation und Experiment entscheidend von $\phi_{S B}$ abhängt.

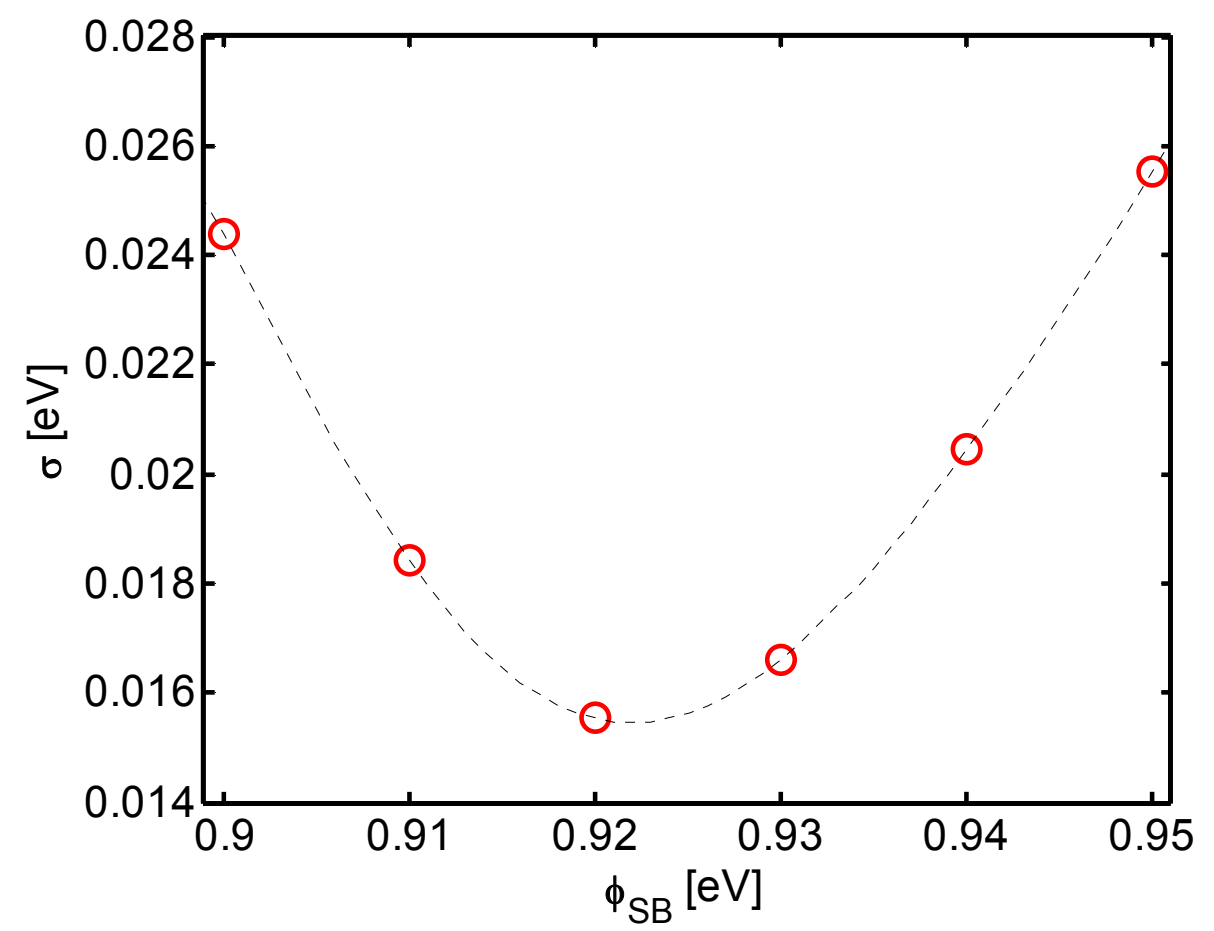

Abbildung 56: Abweichung zwischen den Messdaten $e U_{\text {Bias }}\left(x, I_{T}=10 p A\right)$ und simulierten Verläufen von $e U_{B i a s}\left(x, \phi_{S B}, \phi_{V T}=k o n s t ., d=8 \mathrm{Ang}\right)$, die für unterschiedliche $\phi_{S B}$ bei einem festen Tunnelabstand berechnet wurden. Um die geringstmögliche Abweichung zwischen Simulation und Experiment zu gewährleisten, wurde $\phi_{V T}$ jeweils optimiert. Zur Bestimmung wurden ausschließlich die Bereiche des experimentellen Verlaufs verwendet, die nicht erkennbar durch Defekte beeinflusst werden. 
Bei einem Tunnelabstand von $d=8 \AA$ A existiert die geringste Abweichung im Fall einer simulierten SBH von $\phi_{S B}=0,92 \mathrm{eV}$. Es handelt sich hierbei um den in Abbildung 55 gezeigten simulierten Verlauf bei $\phi_{V T}=0,44 \mathrm{eV}$. Um zu überprüfen, in wieweit dieses Ergebnis von dem Tunnelabstand abhängt, wurden Simulationen für $\phi_{S B}=0,92 \mathrm{eV}$ bei unterschiedlichen Spitze-Probe Abständen durchgeführt.

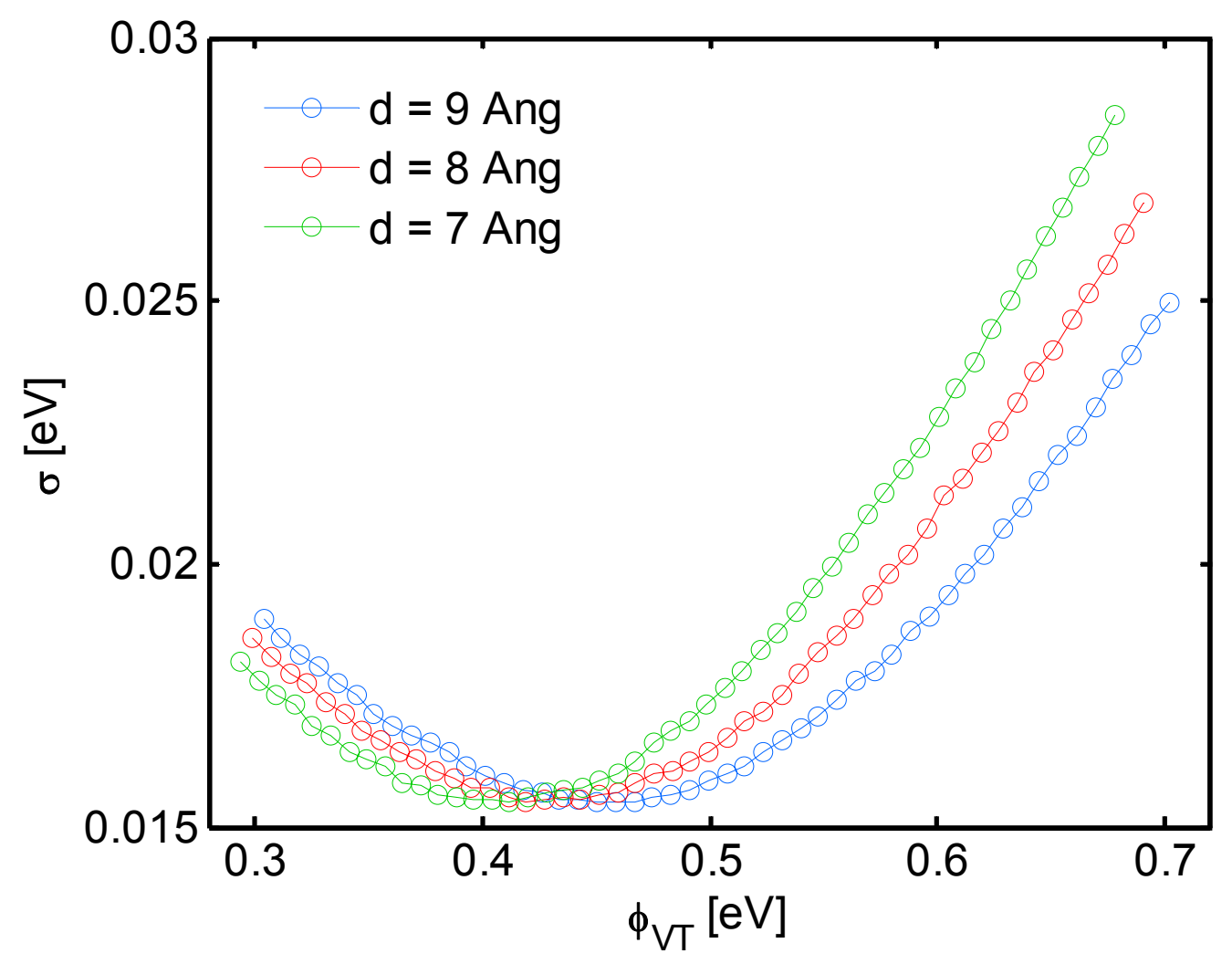

Abbildung 57: Abweichung zwischen den Messdaten $e U_{\text {Bias }}\left(x, I_{T}=10 p A\right)$ und simulierten Verläufen von $e U_{B i a s}\left(x, \phi_{S B}=0,92 \mathrm{eV}, \phi_{V T}, d\right)$, die für variierende $\phi_{V T}$ bei jeweils festen Tunnelabständen von $7 \AA$, $8 \AA$ und $9 \AA$ berechnet wurden.

Aus der Auftragung in Abbildung 57 geht hervor, dass eine Veränderung des simulierten Tunnelabstands im Rahmen der experimentellen Ungenauigkeit (vergleiche Kapitel 2.1) keinen signifikanten Einfluss auf die maximal erreichbare Übereinstimmung hat. Bei einem festen $\phi_{S B}$ existiert offenbar unabhängig von dem Tunnelabstand jeweils ein Energieintervall $\phi_{V T}$, bei dem eine nahezu identische minimale Abweichung zwischen dem experimentellen Verlauf und einem simulierten Verlauf besteht. Gleiches gilt bei einer Veränderung des Öffnungswinkels der Spitze (siehe Abbildung 58), der durch das in Kapitel 6.2 beschriebene Verfahren zum Teil nicht exakt bestimmt werden kann. 


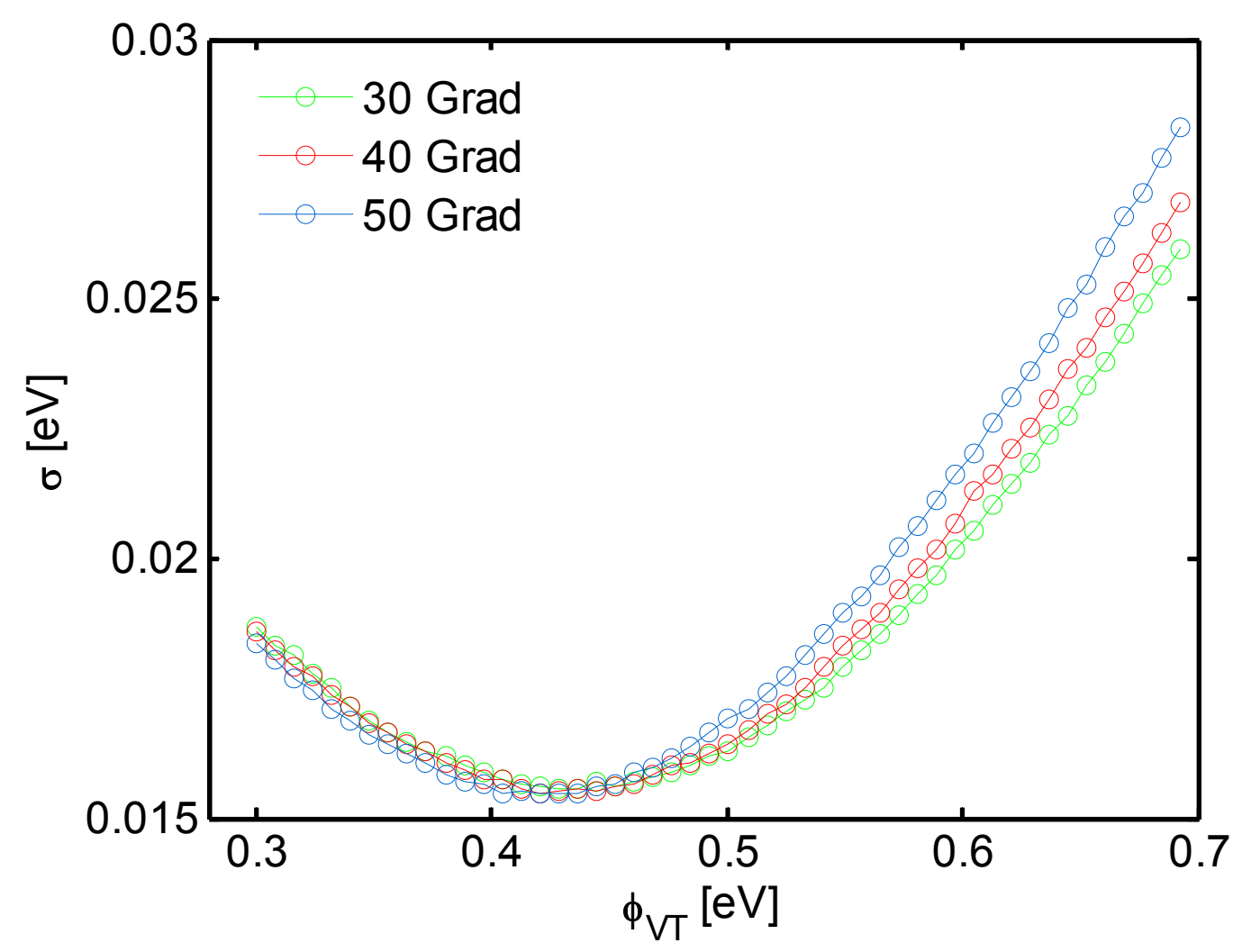

Abbildung 58: Abweichung zwischen den Messdaten $e U_{\text {Bias }}\left(x, I_{T}=10 p A\right)$ und simulierten Verläufen von $e U_{B i a s}\left(x, \phi_{S B}=0,92 \mathrm{eV}, \phi_{V T}, d=8 \mathrm{Ang}\right)$, die für variierende $\phi_{V T}$ bei unterschiedlichen Öffnungswinkeln der STM Spitze berechnet wurden.

Anhand von Abbildung 57 und Abbildung 58 wird deutlich, dass die Übereinstimmung zwischen dem experimentellen und dem simulierten Verlauf weder von dem exakten Tunnelabstand $d$ noch von dem Öffnungswinkel der Spitze signifikant abhängt. Die Unkenntnis dieser beiden Parameter hat folglich keinen Einfluss auf die Bestimmung von $\phi_{S B}$, da die geringste Abweichung stets bei einem $\phi_{S B}$ von 0,92 $\mathrm{eV}$ auftritt. Sie führt lediglich dazu, dass die Spitzenaustrittsarbeit $\phi_{T}$ nicht exakt bestimmt werden kann, die wie beschrieben jeweils aus der konstanten Differenz zwischen dem Verlauf von $\phi_{T S}\left(x, \phi_{S B}, \phi_{V T}, d\right)$ und $e U_{B i a s}\left(x, I_{T}=10 p A\right)$ hervorgeht.

Das im Rahmen dieser Arbeit entwickelte Verfahren erlaubt es demnach, die SBH des untersuchten Fe-GaAs(110) Schottky-Kontakts über die FEM Simulationen der experimentellen XSTS Daten eindeutig zu identifizieren. Der Fehler, mit dem die SBH bestimmt werden kann, hängt zum einen davon ab, wie stark der experimentelle Verlauf $e U_{\text {Bias }}\left(x, I_{T}=10 p A\right)$ um den simulierten idealen Verlauf 
von $e U_{\text {Bias }}\left(x, \phi_{S B}=0,92 \mathrm{eV}\right)$ streut. ${ }^{25}$ Diese Größe wird durch $\sigma$ repräsentiert, dass gemäß (7-10) in Analogie zur Standardabweichung ein Maß für die Streuung der Messwerte um die Funktion $e U_{\text {Bias }}\left(x, \phi_{S B}=0,92 \mathrm{eV}\right)$ darstellt. Zum anderen hängt der Fehler davon ab, wie stark $\phi_{S B}$ den simulierten Verlauf von $e U_{B i a s}\left(x, \phi_{S B}\right)$ verändert.

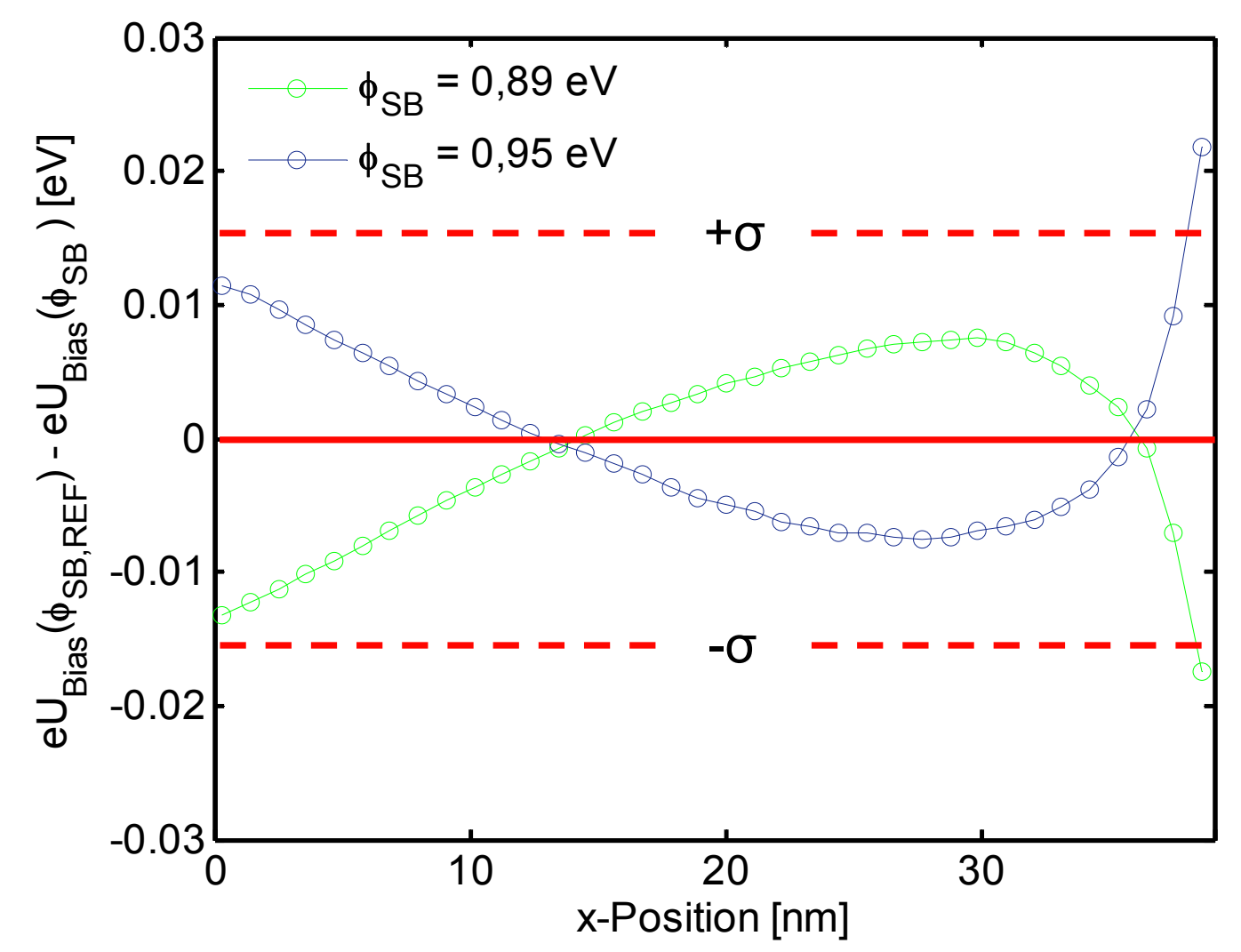

Abbildung 59: Differenz zwischen dem idealen Verlauf von $e U_{B i a s}\left(x, \phi_{S B}=0,92 \mathrm{eV}\right)$ und simulierten Verläufen für $\phi_{S B}=0,89 \mathrm{eV}$ und $\phi_{S B}=0,95 \mathrm{eV}$. Der Bereich von $\pm \sigma=0,0155 \mathrm{eV}$ ist angedeutet.

Aus Abbildung 59 geht hervor, dass eine Veränderung der simulierten $\mathrm{SBH}$ um $\pm 0,03 \mathrm{eV}$ bereits dazu führt, dass die Abweichung zwischen den simulierten Verläufen und dem Referenzverlauf den Bereich von $\pm \sigma$ überschreitet. Die SBH des idealen Fe-GaAs(110) Schottky-Kontakts beträgt demnach $0,92(3) \mathrm{eV}$.

Sie liegt damit deutlich über den Werten von konventionell bei RT gewachsenen Fe-GaAs $\{110\}$ Schottky-Kontakten, bei denen SBHs von $0,74-0,78 \mathrm{eV}$ gemessen wurden [138, 139]. Mönch

\footnotetext{
${ }^{25}$ Bei den im Folgenden angegebenen simulierten Verläufen $e U_{B i a s}\left(x, \phi_{S B}\right)$ handelt es sich stets um die Verläufe bei dem $\phi_{V T}$, bei dem die geringeste Abweichung zum experimentellen Verlauf auftritt.
} 
konnte zunächst am Beispiel von Metall-Silizium und Silizid-Silizium Schottky-Kontakten zeigen, dass derartige Unterschiede in den SBHs in einem direkten Zusammenhang mit der Defektdichte in der Grenzflächenregion stehen [19]:

- Im Fall einer hohen Defektdichte wird die Ausprägung der SBH durch Defektzustände dominiert und Defekt-Modelle wie das in Kapitel 1.5 diskutierte UDM/AUDM sind relevant.

- Bei idealen Schottky-Kontakten mit abrupten, homogenen Grenzflächen führen MIGS zu dem experimentell beobachteten FLP (Kapitel 1.4).

In einer späteren Arbeit hat Mönch die Relevanz dieses Modell auch im Fall von Metall-GaAs $\{110\}$ nachweisen können [140-142]. Demnach wird die SBH des idealen Fe-GaAs(110) Schottky-Kontakts alleine durch die Eigenschaften der MIGS sowie die Differenz der Elektonegativitäten von Substrat und Metallfilm bestimmt. Enstprechend der Lage des CNL des GaAs Substrats (0,50 eV über der Valenzbandkante [59]) sollte die SBH des Fe-GaAs(110) Schottky-Kontakts bei RT etwa 0,95 eV betragen. Die experimentell bestimmte $\mathrm{SBH}$ von 0,92(3) $\mathrm{eV}$ stimmt sehr gut mit dem theoretischen Wert des MIGS Modells überein. Der deutlich geringere Wert von 0,74-0,78 eV, der bei konventionell gewachsenen Fe-GaAs\{110 Schottky-Kontakten bestimmt wurde, wird in dem von Mönch entwickelten Modell auf den Einfluss von Defekten im Bereich der Grenzfläche zurückgeführt [140]. Die Existenz dieser Defekte konnte bereits in verschiedenen Untersuchungen des konventionellen epitaktischen Wachstums von Fe-GaAs\{110\} Systemen nachgewiesen werden (siehe Kapitel 5.1).

Die Bestimmung der SBH des idealen Fe-GaAs(110) Schottky-Kontakts bestätigt das von Mönch entwickelte Modell, in dem MIGS als primäre Ursache des FLP identifiziert werden [18, 142]. Neben den MIGS spielen sekundäre Effekte wie z.B. präparationsbedingte Defekte oder die Bildung von neuen Phasen im Bereich der Grenzfläche ebenfalls eine wichige Rolle bei der Ausprägung der SBH, da sie zu starken Abweichungen von dem MIGS Modell führen können.

\subsubsection{Variation des lokalen elektrostatischen Potentials in der Raumla- dungszone}

Bei der Beschreibung des Potentialverlaufs in der RLZ eines Schottky-Kontakts wird üblicherweise angenommen, dass die Ladungsdichte der ionisierten Dotieratome als homogen betrachtet werden kann. In verschiedenen theoretischen Arbeiten wurde bereits darauf hingewiesen, dass die diskrete Verteilung von Dotieratomen in der Verarmungszone einen signifikanten Einfluss auf die effektive SBH haben kann, da selbst bei geringen Dotierkonzentrationen Bereiche der Barriere so schmal werden können, dass Tunnelprozesse relevant werden [27, 28]. Dies führt dazu, dass die SBH selbst bei idealen Schottky-Kontakten mit einer abrupten homogenen Grenzfläche laterale Inhomogenitäten 
aufweist. Bei der Charakterisierung einer Schottky-Barriere ist es daher essentiell, auch die Veränderungen des lokalen elektrostatischen Potentials bestimmen zu können, die durch die diskrete Verteilung der Dotieratome hervorgerufen werden.

Kürzlich konnte bereits am Beispiel von n-GaAs Spaltflächen gezeigt werden, dass derartige Veränderungen mit dem XSTM bestimmt werden können [95, 137]. In diesem Fall wurden LT-XSTS Messungen dazu verwendet, um anhand des Ionisationsprozesses eines oberflächennahen Donators die lokale Variation der Oberflächenbandverbiegung zu bestimmen, die durch das Dotieratom hervorgerufen wird. Da Ionistationsprozesse im Fall der RT-XSTS Messungen aus dem Bereich der RLZ eines Schottky-Kontakts nicht beobachtet werden, wurde ein neues Verfahren zur Bestimmung der elektrostatischen Potentiallandschaft entwickelt, das im Folgenden vorgestellt wird.

Im Kapitel 7.2 wurde darauf hingewiesen, dass neben den Oberflächendefekten vor allem die Dotieratome zu signifikanten Abweichungen zwischen dem experimentell bestimmten Verlauf von $e U_{B i a s}\left(x, I_{T}=10 p A\right)$ und dem simulierten idealen Verlauf von $e U_{B i a s}\left(x, \phi_{S B}=0,92 \mathrm{eV}\right)$ führen. Abbildung 60 verdeutlicht am Beispiel eines oberflächennahen ionisierten Donators, dass die Abweichungen in diesem Fall in Analogie zu den Ausführungen in Ref. [95, 137] durch Veränderungen des lokalen elektrostatischen Potentials hervorgerufen werden.

(a)

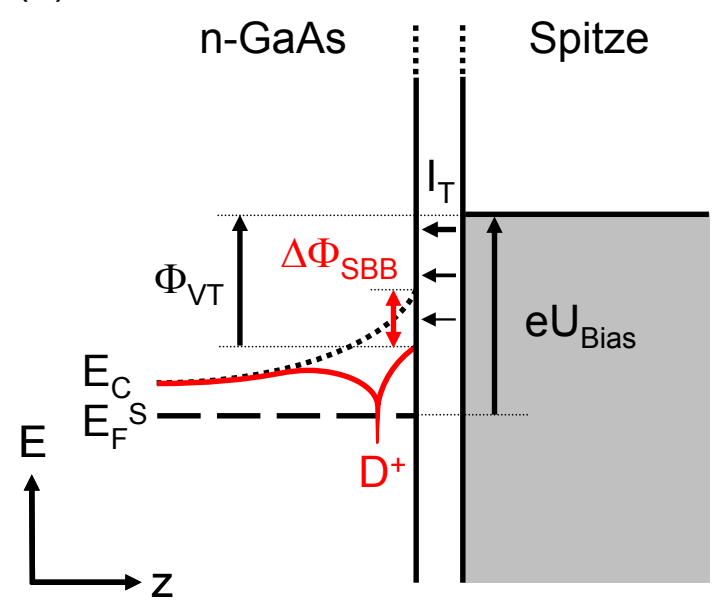

(b)

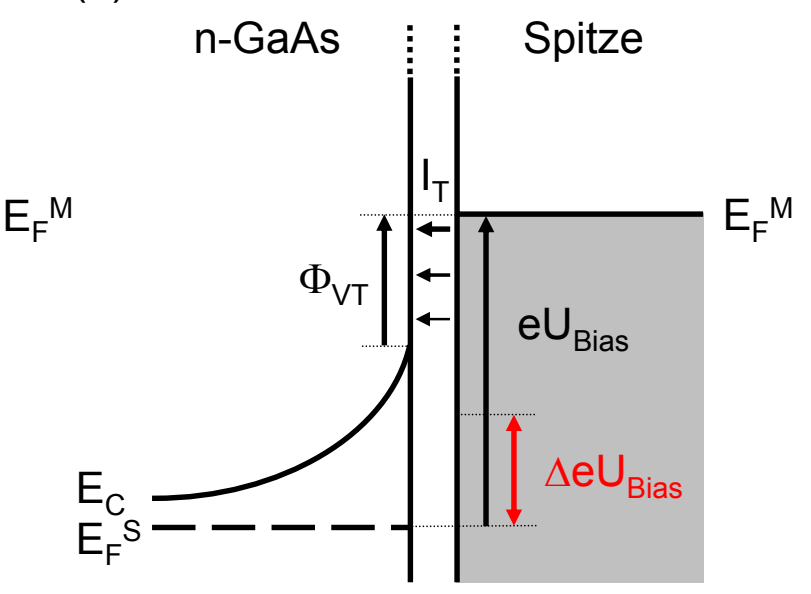

Abbildung 60: (a) Schematische Darstellung des Tunnelkontakts unter dem Einfluss eines ionisierten Donators $\left(D^{+}\right)$. Im Bereich des n-GaAs Substrats ist der Verlauf der Leitungsbandkante skizziert, der durch den Donator verändert wird (Differenz zwischen der gestrichelten schwarzen und der durchgezogenen roten Linie). (b) Ohne den Einfluss des Dotieratoms muss eine höhere Bias-Spannung angelegt werden, um dasselbe Tunnelintervall $\phi_{V T}$ zu erreichen. 
Durch ein ionisiertes Dotieratom wird unter anderem die Oberflächenbandverbiegung $\phi_{S B B}$ im Bereich des Tunnelkontakts verändert. Im Fall des in Abbildung 60 (a) gezeigten Donators vergrößert sich dadurch das Energieintervall $\phi_{V T}$. Um in der in Abbildung 60 (b) dargestellten Situation ohne den Einfluss des Donators dasselbe Intervall $\phi_{V T}$ und damit denselben Tunnelstrom zu erreichen, muss eine höhere Bias-Spannung angelegt werden, so dass der Verlauf von $e U_{B i a s}\left(x, I_{T}=\right.$ konst.) lokal von dem idealen simulierten Verlauf abweicht. Ein Vergleich der Verläufe in Abbildung 55 zeigt, dass es sich hierbei um eine signifikante Abweichung handelt, die selbst in einem Abstand von einigen Nanometern zum Dotieratom noch deutlich zu erkennen ist.

Anhand der beiden Skizzen in Abbildung 60 ist zu erkennen, dass die zu bestimmende Änderung $\Delta \phi_{S B B}$ der elektrostatischen Energie an der Oberfläche des Substrats, die durch das Dotieratom hervorgerufen wird, nicht dem $\Delta e U_{\text {Bias }}$ zwischen dem simulierten und dem experimentellen Verlauf entspricht. Die Differenz wird durch das unterschiedliche TIBB hervorgerufen, das sich in Abhängigkeit von der Bias-Spannung verändert. Um $\Delta \phi_{S B B}(x, y)$ bestimmen zu können, ist es daher notwendig, $\phi_{\text {TIBB }}\left(U_{\text {Bias }}\right)$ in Abhängigkeit von dem Abstand zum Metallfilm mit Hilfe der FEM Simulation zu berechnen, so dass das experimentell zugängliche $\Delta e U_{\text {Bias }}(x, y)$ entsprechend korrigiert werden kann.

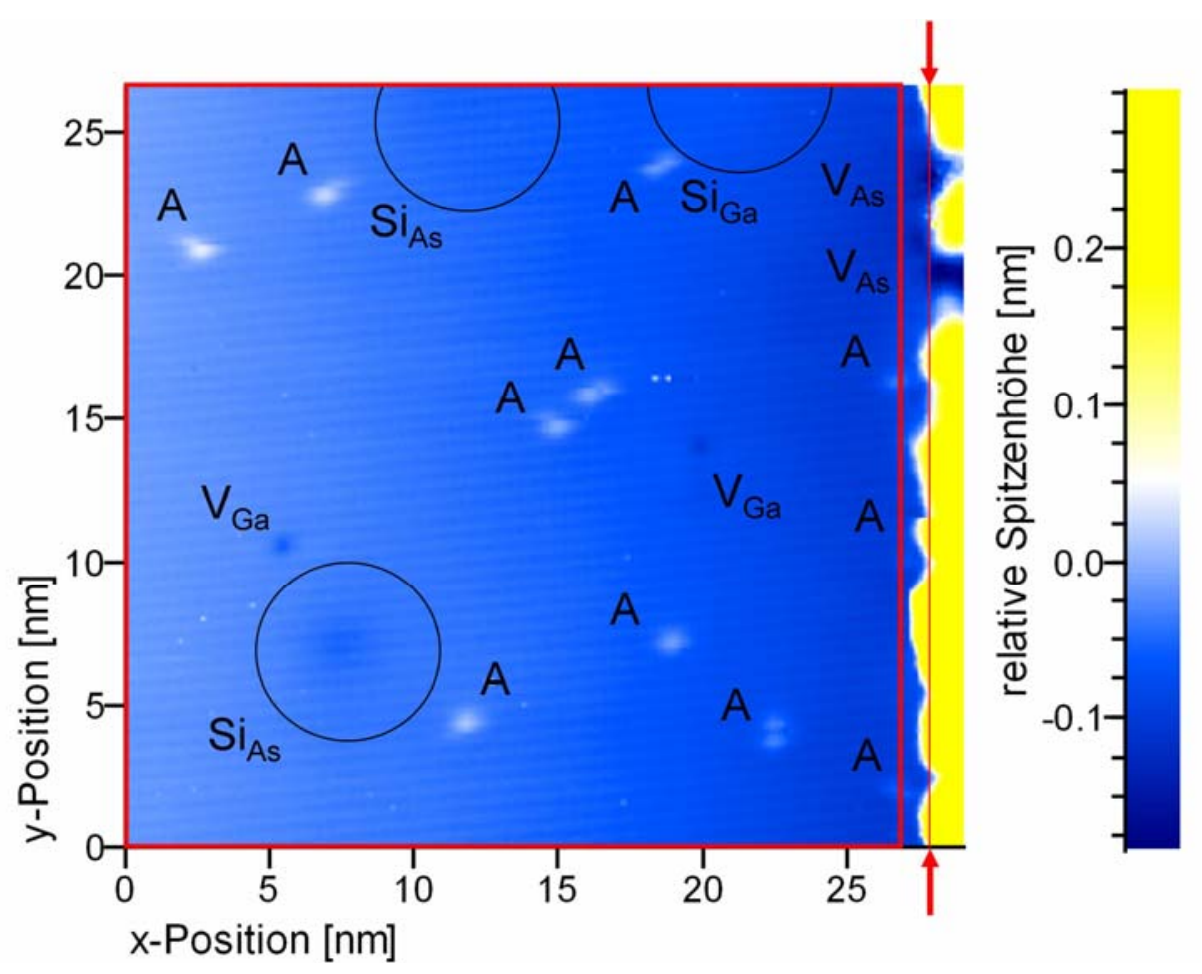


Abbildung 61: XSTM Topografie $\left(U_{\text {Bias }}=2,0\right.$ V, $I_{T}=100$ pA) aus dem Bereich der RLZ. Die Position der Metall-Halbleiter Grenzfläche ist angegeben. Die auftretenden Defekte, die zum Teil in der Topografie kaum zu erkennen sind, wurden wie in Kapitel 6.4.1 beschrieben anhand einer Multi-Bias Messung desselben Ausschnitts identifiziert.

Abbildung 61 zeigt zunächst die XSTM Topografie des Bereichs der Spaltfläche, der spektroskopisch untersucht wurde. Der Ausschnitt erstreckt sich von der RLZ über die markierte Grenzfläche bis zu dem Fe-Film. Im Bereich des GaAs Substrats sind die verschiedenen auftretenden Defekte markiert. Neben den häufig auftretenden Adatomen (A), deren Positionen sich zum Teil wie in Kapitel 6.4.1 beschrieben während der Messung leicht verändern, werden atomare As- und Ga-Fehlstellen $\left(\mathrm{V}_{\mathrm{As}}\right.$, $\mathrm{V}_{\mathrm{Ga}}$ ) sowie die Signaturen von oberflächennahen Si-Dotieratomen beobachtet. Es handelt sich hierbei um zwei $\mathrm{Si}_{\mathrm{As}}$-Akzeptoren und ein $\mathrm{Si}_{\mathrm{Ga}}$-Donator. Insbesondere die ausgedehnte Signatur des $\mathrm{Si}_{\mathrm{As}}$-Akzeptors in der linken unteren Ecke des gezeigten Ausschnitts ist deutlich in der Topografie zu erkennen. Abbildung 62 zeigt die entsprechende lokale Veränderung der Oberflächenbandverbiegung $\Delta \phi_{S B B}(x, y)$ in dem Bereich, der in Abbildung 61 durch den roten Rahmen markiert wird.

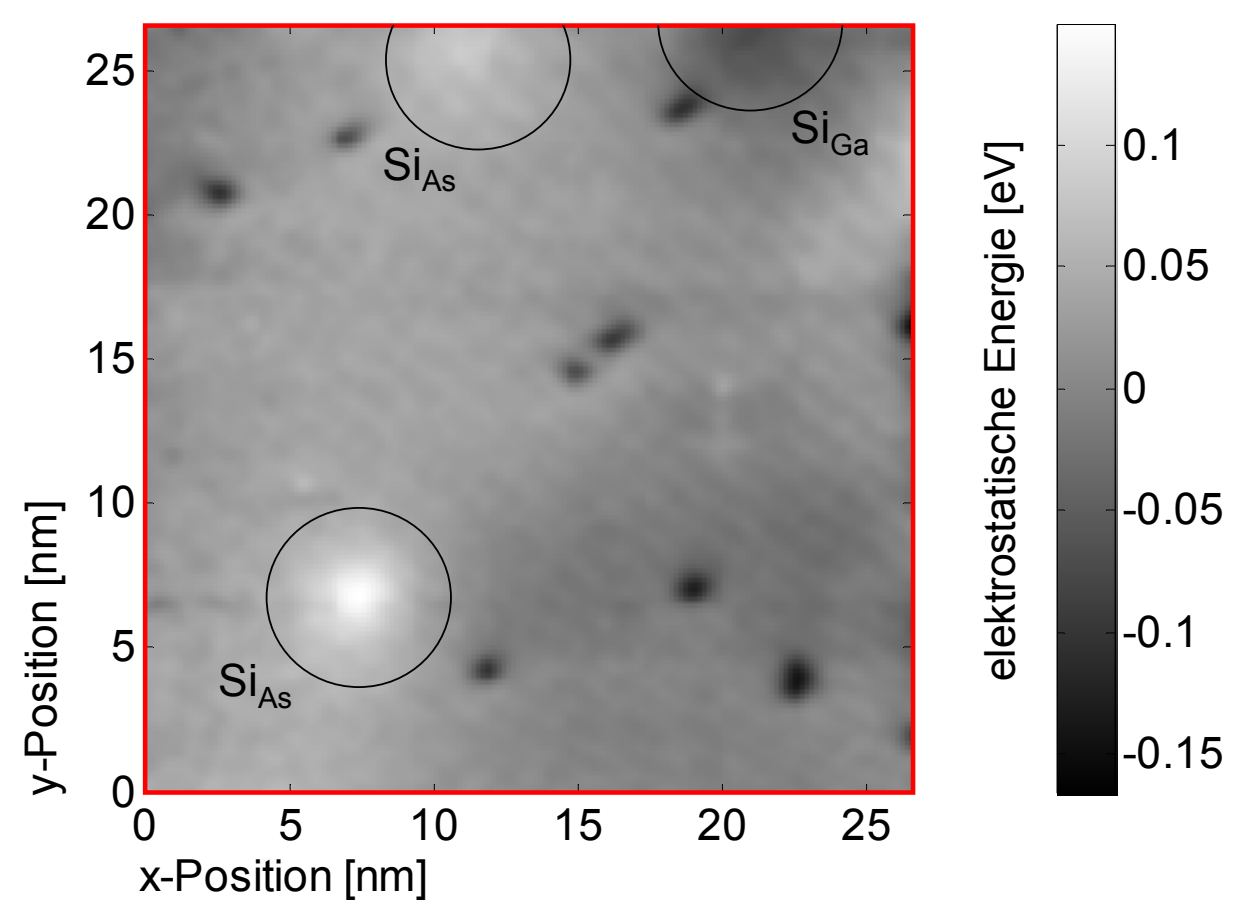

Abbildung 62: $\Delta \phi_{S B B}(x, y)$ in dem rot markierten Bereich in Abbildung 61. Der Einfluss der Dotieratome auf die elektrostatische Energie an der Oberfläche des Substrats ist deutlich zu erkennen. Im Gegensatz zu der Topografie, in der die Dotieratome nur schwache Signaturen hinterlassen, ist der Einfluss auf die lokale elektrostatische Energie deutlich zu erkennen. Im Fall des $\mathrm{Si}_{\mathrm{Ga}}$-Donators, der sich einige Monolagen unterhalb der GaAs(110) Spaltfläche befindet, kommt es wie anhand von Abbildung 60 erläutert zu einer Absenkung um etwa $75 \mathrm{meV}$. Der näher an der Substratoberfläche 
befindliche $\mathrm{Si}_{\mathrm{As}}$-Akzeptor in der linken unteren Ecke führt im Gegensatz dazu wie erwartet zu einer Erhöhung der elektrostatischen Energie, die maximal etwa $150 \mathrm{meV}$ beträgt. Diese Veränderungen sind in sehr guter Übereinstimmung mit dem maximalen $\Delta \phi_{S B B}(x, y)$ von etwa $220 \mathrm{meV}$, dass im Fall eines $4 \mathrm{ML}$ unter der Spaltfläche befindlichen $\mathrm{Si}_{\mathrm{Ga}}$-Donators anhand des Ionisierungsprozesses bestimmt wurde [95, 137]. Dies deutet an, dass sich die hier untersuchten Dotieratome mindestens 5 ML unter der Oberfläche befunden haben. Die Daten in Abbildung 62 lassen darüber hinaus darauf schließen, dass sich weitere $\mathrm{Si}_{\mathrm{Ga}}$-Donatoren im rechten unteren Bereich sowie in der linken oberen Ecke des untersuchten Ausschnitts befunden haben, die anhand der XSTM Topografie nicht identifiziert werden konnten.

Da die durch den zweiten Spaltvorgang hervorgerufenen extrinsischen Oberflächendefekte (Adatome, Fehlstellen) in der RLZ ungeladen sind, wird die Bestimmung der elektrostatischen Potentiallandschaft des Fe-GaAs(1110) Schottky-Kontakts nur in der direkten Umgebung dieser Defekte beeinträchtigt. Die lokale elektrostatische Energie $\phi(x, y)$, die in Abbildung 63 dargestellt ist, entspricht der Summe aus dem selbstkonsistent berechneten $\phi_{R L Z}\left(x, \phi_{S B}=0,92 \mathrm{eV}\right)$ und dem in Abbildung 62 gezeigten $\Delta \phi_{S B B}(x, y)$. Die bislang nur theoretisch beschriebene natürliche Inhomogenität der Potentiallandschaft in der RLZ, die durch die zufällige Verteilung der Dotieratome sowie die Art des Einbaus in das Kristallgitter des Substrats hervorgerufen wird, ist deutlich zu erkennen. Die Fluktuationen in der Nähe der Grenzfläche werden durch die Adatome sowie die As-Fehlstellen hervorgerufen, die sich am Rand des gezeigten Ausschnitts befinden (siehe Topografie in Abbildung 61). 


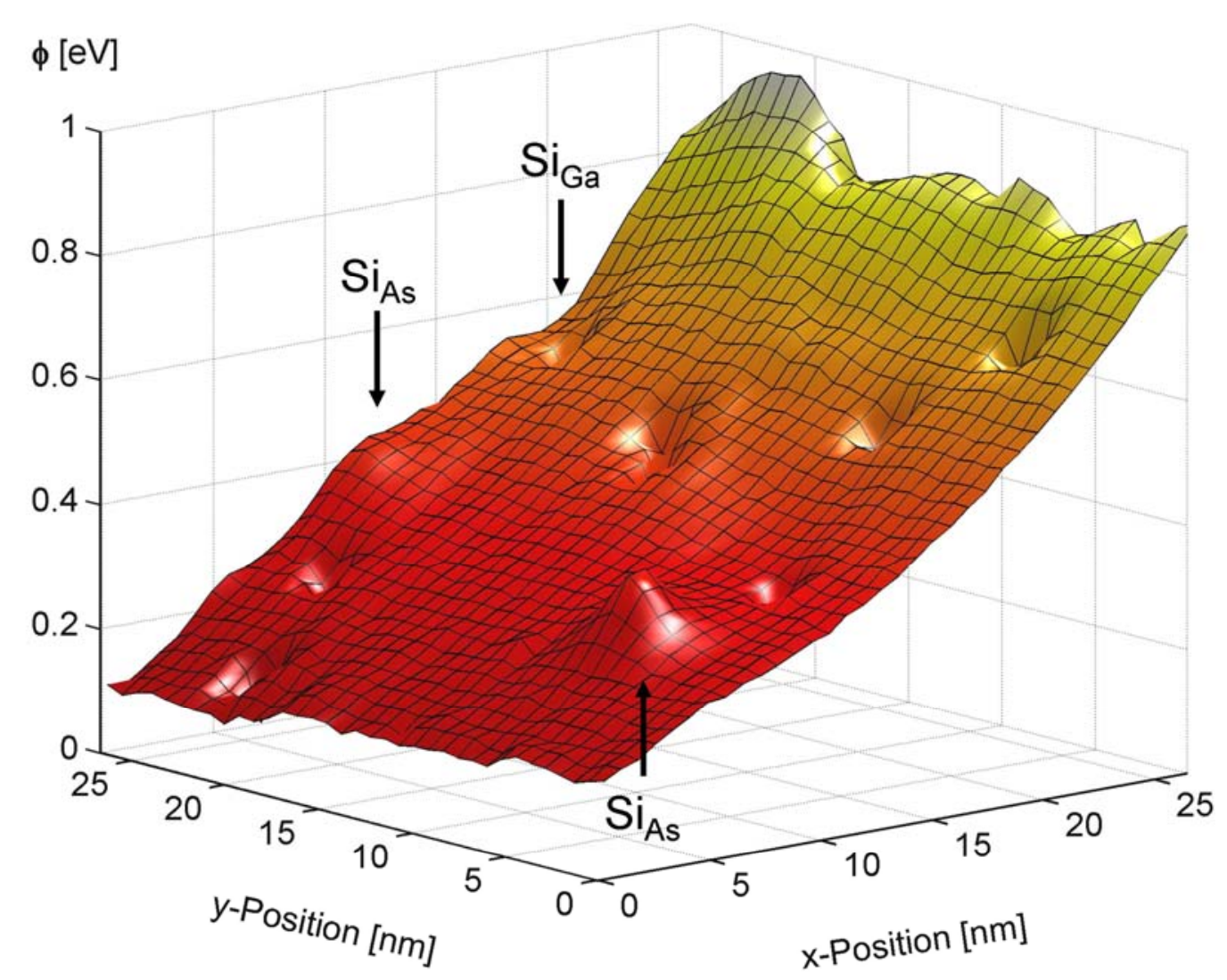

Abbildung 63: 3D-Darstellung von $\phi(x, y)$ in dem durch den roten Rahmen gekennzeichneten Bereich in Abbildung 61, der etwa 1,1 nm vor der Metall-Halbleiter Grenzfläche endet.

Der verwendete Zugang über das XSTM kann demnach nicht nur wertvolle Informationen über die charakteristischen strukturellen Eigenschaften eines Schottky-Kontakts liefern, auf die in Kapitel 6.4 eingegangen wurde. Darüber hinaus bieten XSTS Messungen in Verbindung mit den FEM Simulationen erstmals die Möglichkeit, die Auswirkungen von strukturellen Defekten auf den Verlauf des lokalen elektrostatischen Potentials in der Ebene der Spaltfläche zu quantifizieren.

\subsection{I (U)-Spektroskopie im Bereich der Metall-Halbleiter Grenzfläche}

In den vorangegangen Kapiteln lag der Fokus auf dem Verständnis und der Auswertung von XSTS Daten des Fe-GaAs(110) Schottky-Kontakts, die im Bereich der RLZ aufgenommen wurden, wobei das direkt an den Fe-Film angrenzende Gebiet stets ausgeklammert wurde. Es handelt sich hierbei um den Bereich, in dem im Rahmen der verschiedenen diskutierten Modelle der SchottkyBarrierenformierung die Existenz von Bandlückenzuständen vorhergesagt wird (siehe Kapitel 1). Da verschiedene physikalische Ursachen diskutiert werden, spielen unter anderem der Nachweis von 
Bandlückenzuständen sowie die Bestimmung des Verlaufs ihrer Zustandsdichte im Energiebereich der Bandlücke eine wichtige Rolle, um die Relevanz der theoretischen Modelle bei dem untersuchten System zu überprüfen.

Um den Metall-Halbleiter Übergang mit XSTS untersuchen zu können, muss der Metallfilm etwa in der Ebene des GaAs Substrats gespalten worden sein. Die XSTM Topografie in Abbildung 64 (a) zeigt ein Bereich der Spaltfläche, in dem dies gelungen ist. Lediglich in der rechten oberen Ecke ragt der Metallfilm weniger als $1 \AA$ über die Spaltebene des GaAs Substrats hinaus. Die atomare Struktur des Substrats ist daher in dem gesamten Ausschnitt mit der STM Spitze zugänglich, ohne dass es zu dem in Kapitel 6.1 geschilderten Abbildungsarefakt kommt. In der Grenzflächenlage des GaAs Substrats können zwei As-Fehlstellen identifiziert werden, die durch den zweiten Spaltvorgang hervorgerufen wurden (siehe Kapitel 6.4).

(a)
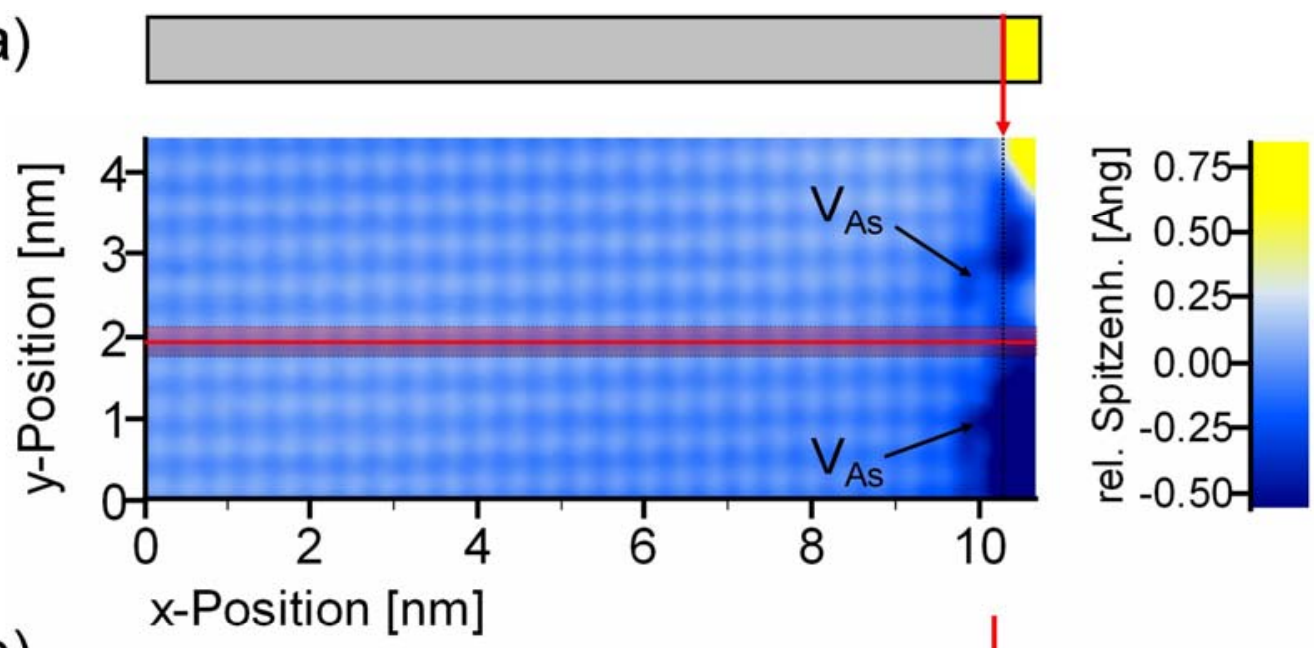

(b)

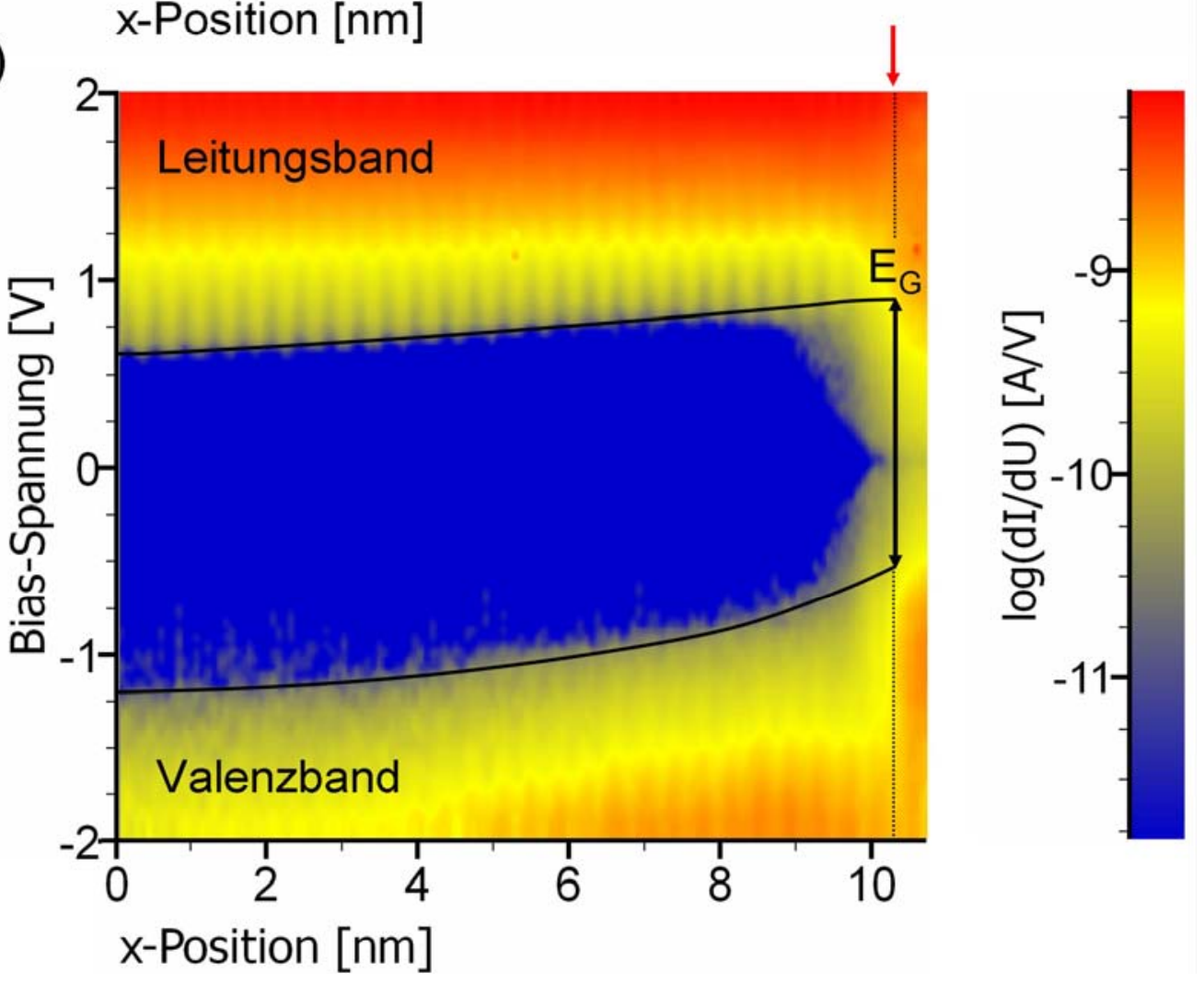


Abbildung 64: (a) Hochaufgelöste XSTM Topografie des Metall-Halbleiter Übergangs $\left(U_{\text {Bias }}=2,0 \mathrm{~V}, I_{T}=100 \mathrm{pA}\right)$. Die Position der Grenzfläche ist angegeben. Direkt an der Grenzfläche befinden sich zwei As-Fehlstellen $\left(V_{A s}\right)$. (b) Logarithmische Auftragung der differentiellen Leitfähigkeit $d I / d U\left(U_{\text {Bias }}\right)$ entlang eines senkrecht auf den Fe-Film zulaufenden Spektrenschnittes. Die rote Linie in (a) markiert die Position des Schnittes. Parallel zur Grenzfläche wurde jeweils über 3 Spektren gemittelt. $E_{G}$ gibt die energetische Lage der GaAs Volumenbandlücke an der Grenzfläche bei einer SBH von $\phi_{S B}=0,92 \mathrm{eV}$ an.

Abbildung 64 (b) zeigt farbkodiert die differentielle Leitfähigkeit $d I / d U\left(U_{\text {Bias }}\right)$ entlang eines senkrecht auf den Metallfilm zulaufenden Schnittes. Die Position des Schnittes, die durch die rote Linie in Abbildung 64 (a) markiert wird, wurde so gewählt, dass der Einfluss der Defekte auf die Spektroskopie minimal ist. Den Ausführungen in Kapitel 2.4 folgend ist die differentielle Leitfähigkeit ein qualitatives Maß für die lokale Zustandsdichte der Probe. Bei den Spektren aus dem Bereich des GaAs Substrats existiert jeweils ein Spannungsbereich mit minimaler differentieller Leitfähigkeit (blau). Es handelt sich hierbei um den Bereich, in dem der Tunnelstrom unter die Auflösungsgrenze der Messapparatur gefallen ist. Sowohl bei positiven als auch bei negativen Bias-Spannungen ist der Einsatzpunkt des Tunnelstroms deutlich zu erkennen.

Die durchgezogenen schwarzen Linien deuten bei positiven bzw. negativen Bias-Spannungen die Verläufe der Einsatzpunkte für Tunnelprozesse in das Leitungsband $\left(I_{E S C}\right)$ bzw. aus dem Valenzband $\left(I_{F S V}\right)$ an, wie sie im Rahmen der in Kapitel 7.1 disuktierten IVCHAR und FEM Simulationen ohne die Existenz von Bandlückenzuständen erwartet werden:

- Bei positiven Bias-Spannungen verschiebt sich der Einsatzpunkt mit geringer werdendem Abstand zum Fe-Film zu höheren Spannungen.

- Bei negativen Bias-Spannungen verschiebt sich der Einsatzpunkt mit geringer werdendem Abstand zu niedrigeren Spannungen.

- Der Spannungsbereich mit minimaler differentieller Leitfähigkeit wird kleiner, da das TIBB mit geringer werdendem Abstand zum Metallfilm geringer wird.

- An der Metall-Halbleiter Grenzfläche geht das TIBB nahezu auf Null zurück, so dass die Einsatzpunkte mit der Lage der Bandkanten $E_{C}$ und $E_{V}$ übereinstimmen, die durch die SBH von $\phi_{S B}=0,92 \mathrm{eV}$ sowie die Weite $E_{G}$ der Volumenbandlücke bei RT bestimmt werden (Doppelpfeil in Abbildung 64 (b)). 
Bei Bias-Spannungen oberhalb der Einsatzpunkte wird eine markante Modulation der differentiellen Leitfähgigkeit beobachtet, die durch die atomare Struktur der GaAs\{110\} Spaltfläche bedingt wird (insbesondere bei positiven Bias-Spannungen deutlich zu erkennen).

Abbildung 64 (b) zeigt, dass die Abstandsabhängigkeit der Einsatzpunkte nur bis etwa 1,5 $\mathrm{nm}$ vor dem Metall-Halbleiter Übergang durch die Simulationen beschrieben werden kann. Bei geringeren Abständen treten Veränderungen auf, die nicht mehr im Rahmen von Tunnelprozessen in leere Leitungsbandzustände bzw. aus gefüllten Valenzbandzuständen erklärt werden können, da der Spannungsbereich, in dem experimentell keine Tunnelprozesse beobachtet werden, kleiner als die GaAs Volumenbandlücke wird. Der Tunnelstrom muss in diesem Fall durch Zustände getragen werden, die sich energetisch im Bereich der Bandlücke des GaAs Substrats befinden. Im Gegensatz zu der starken Modulation der differentiellen Leitfähigkeit oberhalb der Einsatzpunkte weist die differentielle Leitfähigkeit dieser Bandlückenzustände keine abstandsabhängige Modulation auf. Anhand des Spektrenschnitts können die Bandlückenzustände folgendermaßen charakterisiert werden:

- Kontinuierlicher Verlauf im gesamten Bereich der Bandlücke

- Die Abklinglänge nimmt von der Mitte der Bandlücke zu den Bandkanten hin zu

- Es existieren keine Energiebereiche mit stark erhöhter differentieller Leitfähigkeit

Diese experimentell bestimmten Eigenschaften der Bandlückenzustände entsprechen denen, die im Rahmen des in Kapitel 1.4 diskutierten MIGS Modells beschrieben wurden. Angesichts des glatten Verlaufs der differentiellen Leitfähigkeit im Energiebereich der GaAs Bandlücke existieren keine Hinweise auf charakteristische Defektzustände, die in dem in Kapitel 1.5 vorgestellten UDM/AUDM für das FLP verantwortlich gemacht werden.

Die XSTS Untersuchung der Bandlückenzustände bestätigt damit die in Kapitel 7.2 gezogenen Schlussfolgerungen. Sowohl die Bestimmung der SBH als auch die charakteristischen Eigenschaften der Bandlückenzustände zeigen übereinstimmend, dass die Physik des idealen Fe-GaAs(1110) Schottky-Kontakts im Rahmen des MIGS Modells zutreffend beschrieben werden kann. 


\section{$8 \quad$ Zusammenfassung}

Im Zentrum der vorliegenden Arbeit stand die Untersuchung des Zusammenspiels zwischen den lokalen strukturellen Eigenschaften und den nanoskaligen elektronischen Eigenschaften von MetallHalbleiter Kontakten. Um derartige Zusammenhänge erstmals im Realraum auf der atomaren Skala studieren zu können, wurde die Methode der Raster-Tunnel-Mikroskopie in Querschnittsgeometrie eingesetzt.

Bei dem untersuchten Probensystem Fe-GaAs handelt es sich um ein prototypisches Modellsystem, das aufgrund seiner nahezu idealen Gitteranpassung seit mehr als 30 Jahren zum Studium der Heteroepitaxie von Metall-Halbleiter Systemen verwendet wird [43, 105, 110]. Die Entwicklung von zukünftigen Spintronik Bauelementen auf der Basis von Ferromagnet-Halbleiter Systemen hat in den vergangenen Jahren das Interesse an Fe-GaAs Schottky-Kontakten noch verstärkt [32, 33, 50].

Um Fe-GaAs Schottky-Kontakte im UHV unter kontrollierten Bedingungen präparieren und anschließend in dem „as prepared“ Zustand mit dem STM in Querschnittsgeometrie untersuchen zu können, wurde ein aufwendiges in-situ Präparationsverfahren entwickelt. Es beruht auf der Möglichkeit, durch Spalten des GaAs Substrats im UHV zunächst eine defektfreie, atomar glatte $\{110\}$ Oberfläche zu erzeugen, auf der der Metallfilm deponiert wird. Zur STM Untersuchung in Querschnittsgeometrie kann der Metall-Halbleiter Kontakt ein zweites Mal über die Grenzfläche hinweg gespalten werden. Am Beispiel eines Bi-GaAs(1110) Schottky-Kontakts wurde gezeigt, dass auf diesem Weg auch im Bereich des Metall-Halbleiter Übergangs eine atomar glatte Oberfläche erzeugt werden kann, die sich ideal zu Untersuchung mit dem XSTM eignet.

Um das epitaktische Wachstum der Fe-GaAs\{110\} Schottky-Kontakte insbesondere im Hinblick auf die Grenzflächeneigenschaften zu optimieren, wurde in Anlehnung an Arbeiten von Smith et al. ein in zwei Schritten ablaufendes Präparationsverfahren entwickelt [111]. Um Reaktionen an der MetallHalbleiter Grenzfläche zu unterdrücken, findet die Deposition des Fe-Films im Gegensatz zu konventionellen Wachstumsprozessen bei einer Temperatur von 130(5) K statt. Anschließend wird die Probe innerhalb von zwei Stunden langsam bis auf RT annealt. STM Untersuchungen der Filmmorphologie, die strukturelle Analyse mit LEED, sowie die Untersuchung der magnetischen Eigenschaften mittels UHV MOKE lassen übereinstimmend darauf schließen, dass dieses Verfahren erstmals die Möglichkeit bietet, ideale Fe-GaAs\{110\} Schottky-Kontakte mit einer abrupten, homogenen Grenzfläche zu wachsen (siehe auch [54]).

Diese Schlussfolgerungen konnten durch die STM Untersuchung eines Fe-GaAs(110) SchottkyKontakts in Querschnittsgeometrie bestätigt werden. Hierbei ist es gelungen, den Übergangsbereich mit atomarer Auflösung abzubilden, so dass die atomare Struktur des GaAs Substrats auf der Basis von XSTM Topografien bis hin zur Metall-Halbleiter Grenzfläche im Realraum aufgeklärt werden 
konnte. Gelegentlich auftretende atomare Defekte in der Oberflächenlage des GaAs Substrats konnten auf den zweiten Spaltvorgang zurückgeführt werden. Da keine Defekte beobachtet wurden, die mit dem Wachstum des Fe-Films in Verbindung stehen, konnte durch die XSTM Messungen bestätigt werden, dass Reaktionen an der Fe-GaAs\{110\} Grenzfläche durch den verwendeten Präparationsablauf effektiv unterdrückt werden können. Dieses Ergebnis ist im Zusammenhang mit der Nutzung von Fe-GaAs Schottky-Kontakten zur Spin-Injektion und -Detektion in zukünftigen Spintronik Bauelementen von besonderem Interesse, da die Effizienz dieser Prozesse durch Defekte im Bereich der Grenzfläche stark beeinträchtigt werden kann [50, 57].

Um den Zusammenhang zwischen den strukturellen Eigenschaften des Fe-GaAs(110) SchottkyKontakts und den lokalen elektronischen Eigenschaften untersuchen zu können, wurden XSTS Messungen durchgeführt. Im Bereich der RLZ des Schottky-Kontakts konnten in Abhängigkeit von dem Abstand zur Metall-Halbleiter Grenzfläche signifikante Veränderungen in den I(U)-Kennlinien beobachtet werden. Aufgrund des experimentell nicht näher bekannten TIBB war es allerdings nicht möglich, elektronische Eigenschaften wie die SBH des Systems oder das lokale elektrostatische Potential in der RLZ direkt aus den spektroskopischen Daten zu extrahieren. Um dies erreichen zu können, wurde im Rahmen dieser Arbeit ein neues, auf der Finite Elemente Methode basierendes Verfahren entwickelt, das die realistische 3D-Simulation des TIBB sowohl unter dem Einfluss des elektrostatischen Potentials in der RLZ als auch unter dem Einfluss des Metallfilms für beliebige Spitzengeometrien erlaubt. Durch den Vergleich von FEM Simulationen mit experimentellen XSTS Daten war es möglich, die SBH des idealen Fe-GaAs(110) Schottky-Kontakts quantitativ zu bestimmen. Sie liegt mit 0,92(3) $\mathrm{eV}$ deutlich über den Werten von $0,74-0,78 \mathrm{eV}$, die bei konventionell gewachsenen FeGaAs $\{110\}$ Schottky-Kontakten gemessen wurden [138, 139]. Die Übereinstimmung mit dem Wert von 0,95 eV, der im Rahmen des MIGS Modells erwartet wird, ist sehr gut [59].

Neben der experimentellen Bestimmung der SBH spielt der Nachweis von Bandlückenzuständen sowie die Bestimmung ihres Verlaufs im Energiebereich der GaAs Bandlücke eine entscheidende Rolle, um die Relevanz theoretischer Modelle der Schottky-Barrierenformierung zu überprüfen. Anhand des Verlaufs der differentiellen Leitfähigkeit im Bereich des Fe-GaAs(110) Übergangs wurde nachgewiesen, dass ein Kontinuum von Bandlückenzuständen existiert, das einen für MIGS charakteristischen Verlauf aufweist [6, 7]. Dies zeigt in Übereinstimmung mit der experimentell bestimmten SBH, dass die elektronischen Eigenschaften des idealen Fe-GaAs(110) Schottky-Kontakts im Rahmen des MIGS Modells zutreffend beschrieben werden können.

Durch die Verbindung von XSTS Daten mit FEM Simulationen des TIBB ist es darüber hinaus erstmals gelungen, die Auswirkungen der diskreten Verteilung von Dotieratomen auf die Potentiallandschaft in der RLZ abzubilden, die bislang nur theoretisch berechnet werden konnte [28]. Im Vergleich zu BEEM Experimenten besitzt die XSTM Technik den Vorteil, dass die Variation des lokalen elektro- 
statischen Potentials direkt mit den verursachenden Defekten verknüpft werden kann. Sie bietet daher ideale Voraussetzungen, um beispielsweise die zu Beginn dieser Arbeit gezeigten Simulationen des elektrostatischen Potentials unter dem Einfluss einzelner Dotieratome experimentell zu überprüfen. 


\section{Anhang A: FEM Simulation des TI BB bei der XSTM Unter- suchung von Schottky-Kontakten}

Die im Folgenden gezeigten Abbildungen verdeutlichen anhand von FEM Simulationen die Abhängigkeit zwischen $\phi_{S B B}\left(x_{\text {Spizze }}\right)$ und relevanten Größen wie der SBH, der Bias-Spannung, dem Spitzenradius, dem Öffnungswinkel der Spitze sowie dem Tunnelabstand. Die Metall-Halbleiter Grenzfläche befindet sich jeweils bei $x=0$.

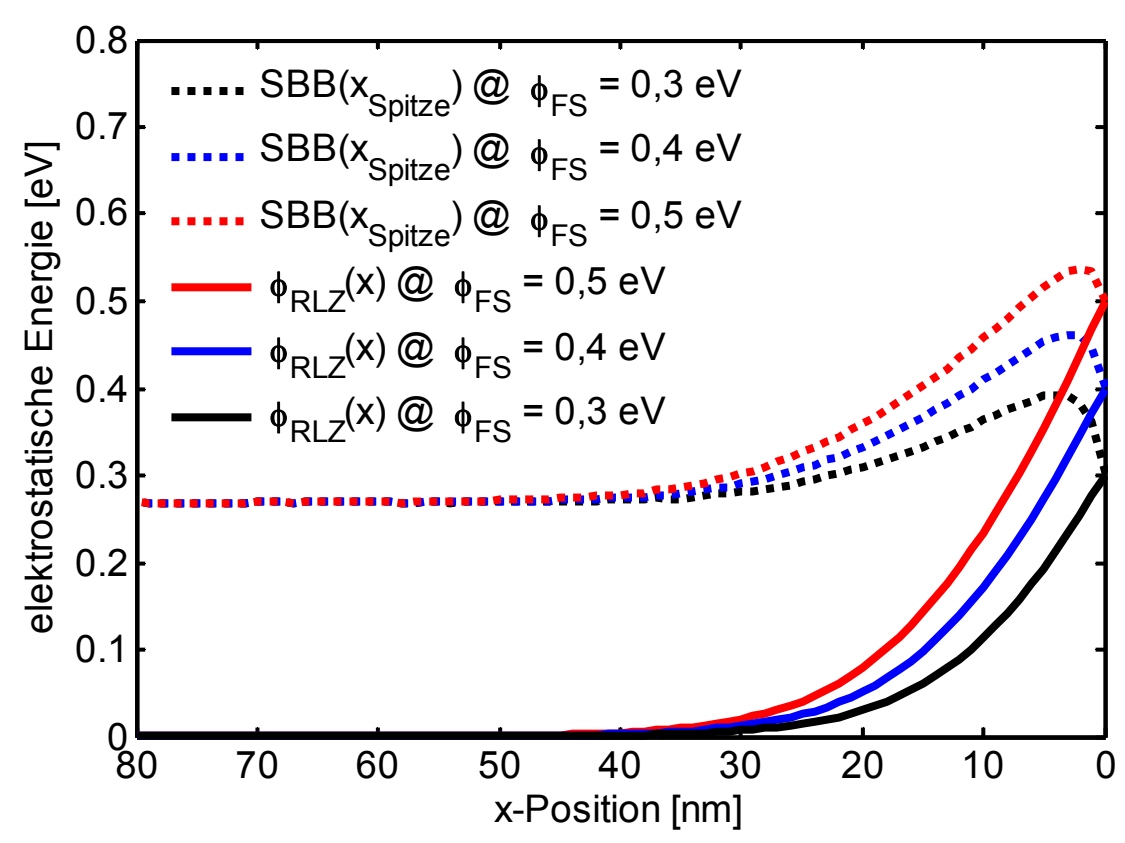

Abbildung 65: $\phi_{S B B}\left(x_{S p i z z e}\right)$ in Abhängigkeit von $\phi_{F S}$, das gemäß Relation (7-6) mit der SBH des Schottky-Kontakts verknüpft ist. Die übrigen Parameter der Simulation sind: $\phi_{T S}=1,0 \mathrm{eV}$, Spitzenradius $10 \mathrm{~nm}$, Öffnungswinkel 40 Grad, Tunnelabstand 8 A. 


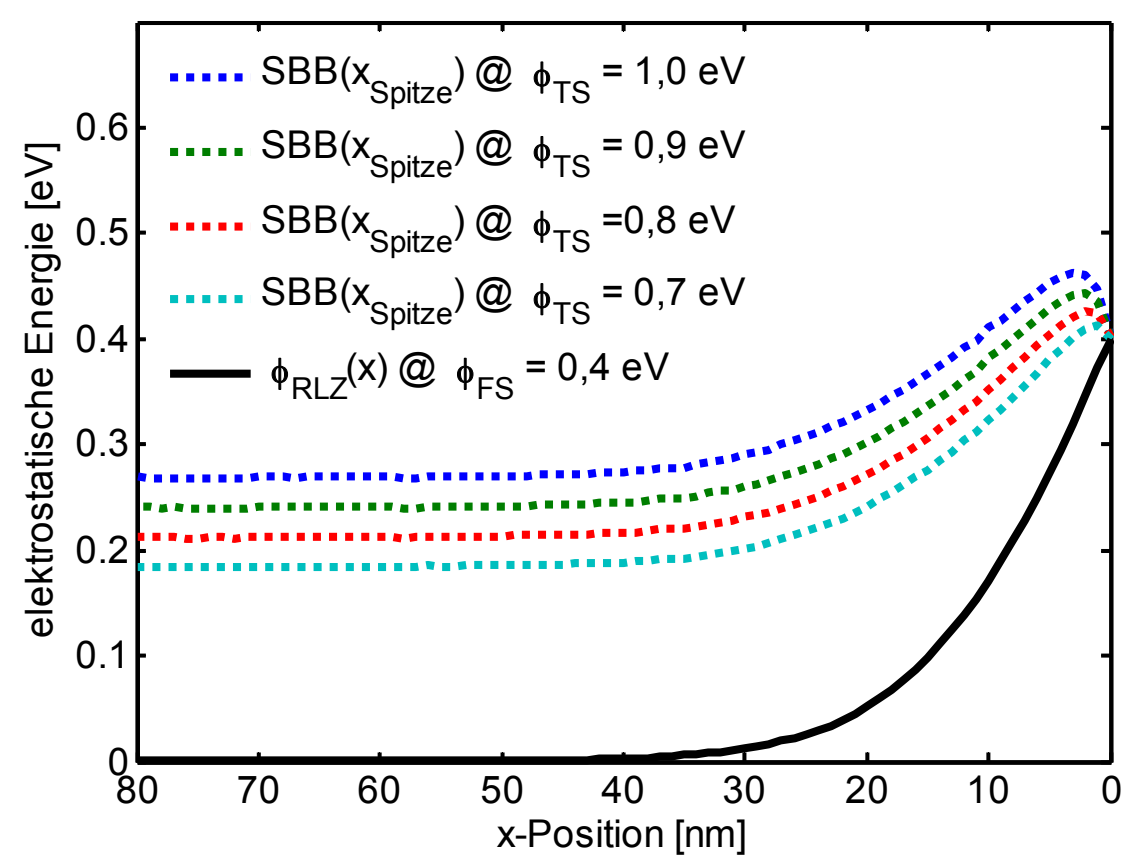

Abbildung 66: $\phi_{S B B}\left(x_{\text {Spitze }}\right)$ in Abhängigkeit von $\phi_{T S}$, das gemäß Relation (7-5) mit der experimentellen Bias-Spannung verknüpft ist. Die übrigen Parameter der Simulation sind: $\phi_{F S}=0,4 \mathrm{eV}$, Spitzenradius $10 \mathrm{~nm}$, Öffnungswinkel 40 Grad, Tunnelabstand 8 A.

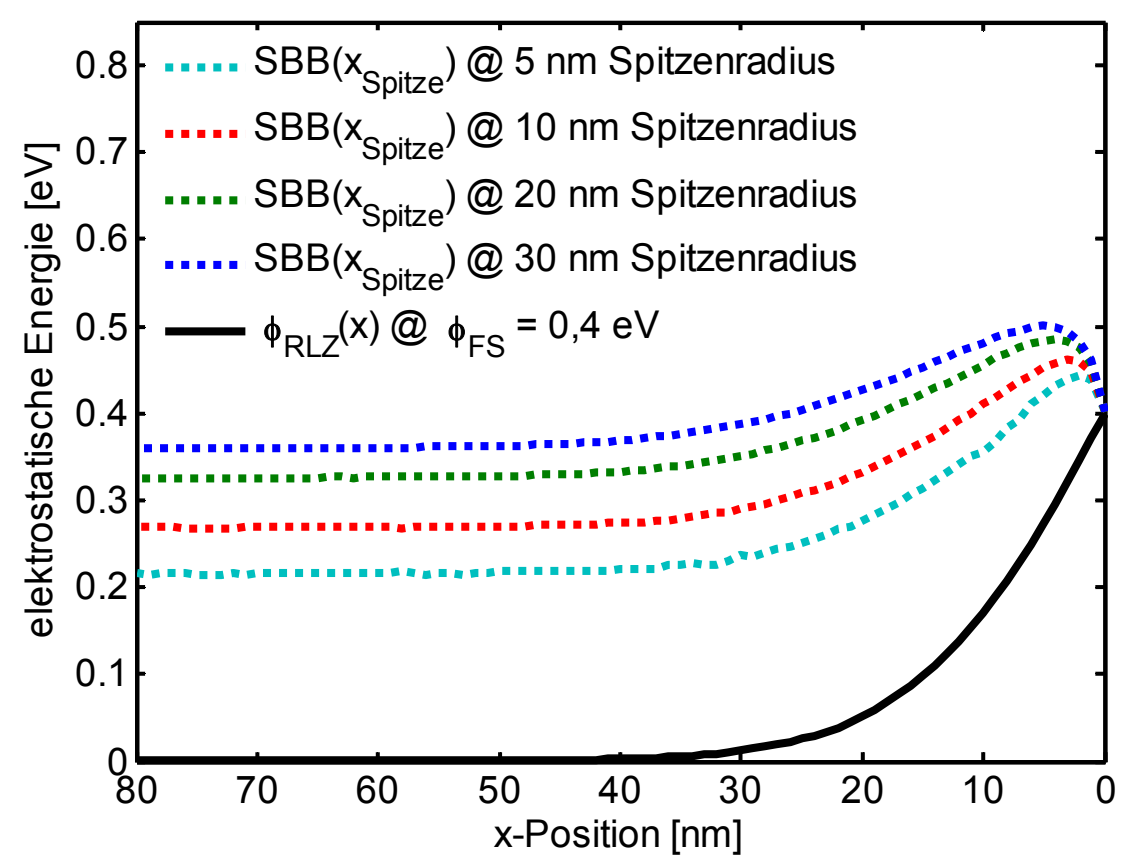

Abbildung 67: $\phi_{S B B}\left(x_{\text {Spitze }}\right)$ in Abhängigkeit von dem Radius der STM Spitze. Die übrigen Parameter der Simulation sind: $\phi_{T S}=1,0 \mathrm{eV}, \phi_{F S}=0,4 \mathrm{eV}$, Öffnungswinkel 40 Grad, Tunnelabstand $8 \AA$. 


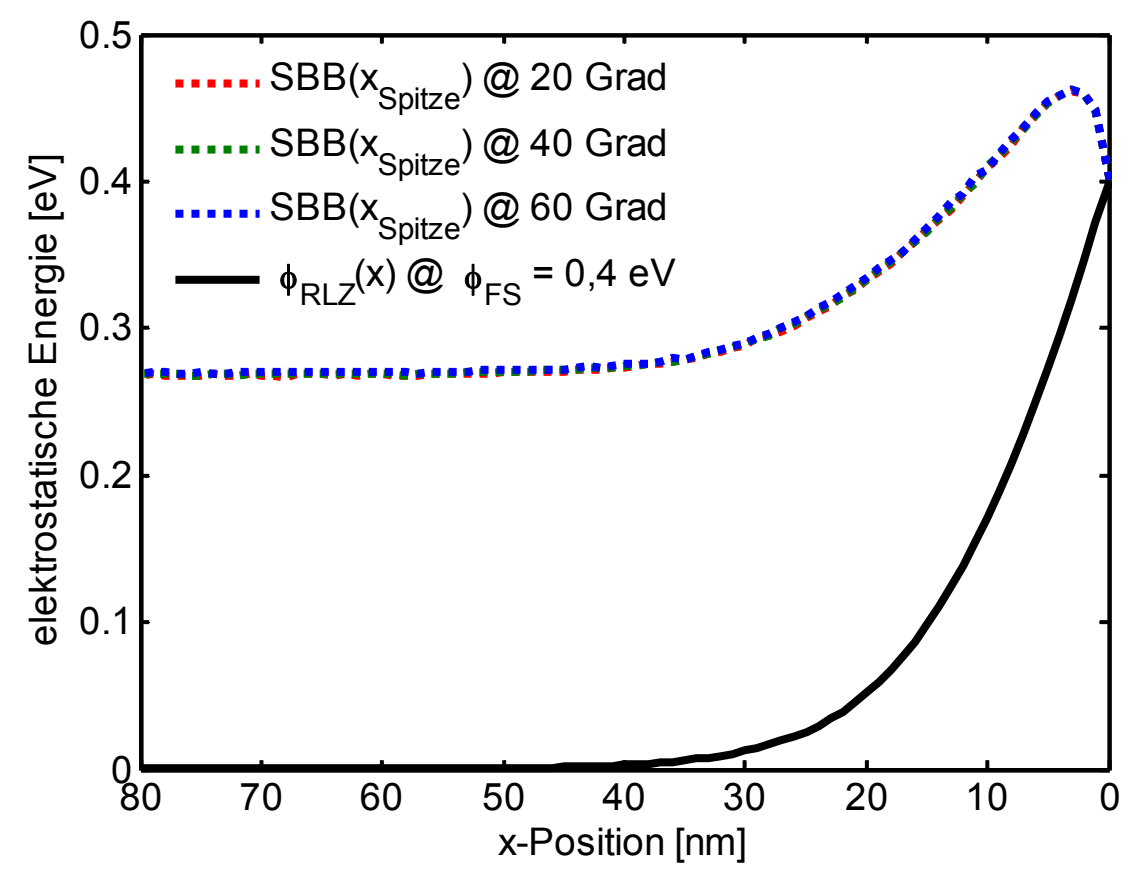

Abbildung 68: $\phi_{S B B}\left(x_{\text {Spitze }}\right)$ in Abhängigkeit von dem Öffnungswinkel der STM Spitze. Die übrigen Parameter der Simulation sind: $\phi_{T S}=1,0 \mathrm{eV}, \phi_{F S}=0,4 \mathrm{eV}$, Spitzenradius $10 \mathrm{~nm}$, Tunnelabstand $8 \AA$.

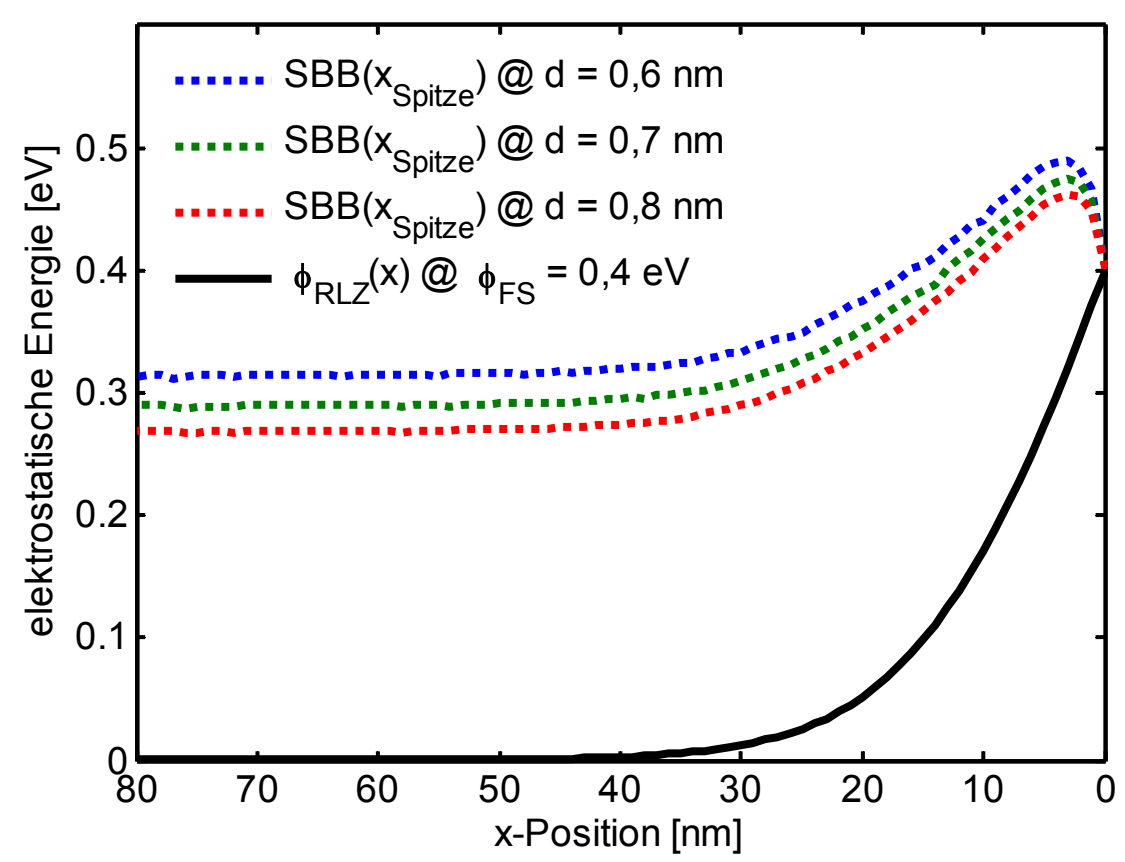

Abbildung 69: $\phi_{S B B}\left(x_{\text {Spitze }}\right)$ in Abhängigkeit von dem Tunnelabstand $d$. Die übrigen Parameter der Simulation sind: $\phi_{T S}=1,0 \mathrm{eV}, \phi_{F S}=0,4 \mathrm{eV}$, Spitzenradius $10 \mathrm{~nm}$, Öffnungswinkel $40 \mathrm{Grad}$. 


\section{Literaturverzeichnis}

[1] F. Braun Pogg. Ann., vol. 153, p. 556, 1874.

[2] W. Monch, “125 years of metal-semiconductor contacts: Where do we stand?,” 1999.

[3] W. Schottky, "Halbleitertheorie der Sperrschicht," Naturwissenschaften, vol. 26, pp. 843-843, Dec. 1938.

[4] N. F. Mott, "Note on the contact between a metal and an insulator or semiconductor," Proc. Cam. Phil. Soc., vol. 34, p. 568, 1938.

[5] J. Bardeen, "Surface states and rectification at a metal semi-conductor contact," Phys. Rev., vol. 71, pp. 717, May 1947.

[6] V. Heine, “Theory of surface states,” Phys. Rev., vol. 138, no. 6A, p. A1689, 1965.

[7] S. G. Louie, J. R. Chelikowsky, and M. L. Cohen, "Ionicity and the theory of schottky barriers," Phys. Rev. B, vol. 15, pp. 2154, Feb. 1977.

[8] J. Tersoff, "Schottky barrier heights and the continuum of gap states," Phys. Rev. Lett., vol. 52, pp. 465, Feb. 1984.

[9] W. E. Spicer, I. Lindau, P. Skeath, C. Y. Su, and P. Chye, "Unified mechanism for schottkybarrier formation and iii-v oxide interface states,” Phys. Rev. Lett., vol. 44, pp. 420, Feb. 1980.

[10] W. E. Spicer, Z. Liliental-Weber, E. Weber, N. Newman, T. Kendelewicz, R. Cao, C. McCants, P. Mahowald, K. Miyano, and I. Lindau, "The advanced unified defect model for schottky barrier formation,” J. Vac. Sci. Technol. B, vol. 6, pp. 1245-1251, July 1988.

[11] R. Ludeke, "Defects and metal states: Towards a predictive model for the schottky barrier," Phys. Rev. B, vol. 40, pp. 1947-, July 1989.

[12] R. T. Tung, "Chemical bonding and fermi level pinning at metal-semiconductor interfaces," Phys. Rev. Lett., vol. 84, no. 26, p. 6078, 2000.

[13] L. J. Brillson, "Interface bonding, chemical reactions, and defect formation at metalsemiconductor interfaces,” J. Vac. Sci. Technol. A, vol. 25, pp. 943-949, July 2007.

[14] L. J. Brillson, “The structure and properties of metal-semiconductor interfaces," Surface Science Reports, vol. 2, pp. 123-326, Nov. 1982.

[15] R. T. Tung, "Schottky-barrier formation at single-crystal metal-semiconductor interfaces," Phys. Rev. Lett., vol. 52, pp. 461, Feb. 1984. 
[16] R. T. Tung, J. M. Gibson, and J. M. Poate, "Formation of ultrathin single-crystal silicide films on Si: Surface and interfacial stabilization of Si-NiSi ${ }_{2}$ epitaxial structures,” Phys. Rev. Lett., vol. 50, pp. 429, Feb. 1983.

[17] R. G. Dandrea and C. B. Duke, "Interfacial atomic composition and schottky barrier heights at the Al/GaAs(001) interface,” J. Vac. Sci. Technol. B, vol. 11, no. 4, p. 1553, 1993.

[18] W. Moench, "Barrier heights of real schottky contacts explained by metal-induced gap states and lateral inhomogeneities,” J. Vac. Sci. Technol. B, vol. 17, pp. 1867-1876, July 1999.

[19] W. Moench, "Role of virtual gap states and defects in metal-semiconductor contacts," Phys. Rev. Lett., vol. 58, pp. 1260-, Mar. 1987.

[20] R. T. Tung, “Recent advances in schottky barrier concepts,” Materials Science and Engineering: R: Reports, vol. 35, pp. 1-138, Nov. 2001.

[21] H. Palm, M. Arbes, and M. Schulz, "Fluctuations of the Au-Si(100) schottky barrier height," Phys. Rev. Lett., vol. 71, pp. 2224, Oct. 1993.

[22] W. J. Kaiser and L. D. Bell, "Direct investigation of subsurface interface electronic structure by ballistic-electron-emission microscopy,” Phys. Rev. Lett., vol. 60, pp. 1406, Apr. 1988.

[23] A. A. Talin, R. S. Williams, B. A. Morgan, K. M. Ring, and K. L. Kavanagh, "Nanometerresolved spatial variations in the schottky barrier height of a Au/n-type GaAs diode,” Phys. Rev. B, vol. 49, pp. 16474, June 1994.

[24] L. J. Brillson, “Metal-semiconductor interfaces,” Surface Science, vol. 299-300, pp. 909-927, Jan. 1994.

[25] G. Moore, “Cramming more components onto integrated circuits,” Electronics Magazine, vol. 38, p. 114, 1965.

[26] “International technology roadmap for semiconductors," 2007.

[27] W. J. Boudville and T. C. McGill, "Resistance fluctuations in ohmic contacts due to discreteness of dopants,” Appl. Phys. Lett., vol. 48, pp. 791-793, Mar. 1986.

[28] V. Bondarenko, Y. Kudinov, S. Ershov, and V. Korablev, "Natural nonuniformities in the height of a schottky barrier,” Semiconductors, vol. 32, pp. 495-496, May 1998.

[29] S. Roy and A. Asenov, “Applied physics: Where do the dopants go?,” Science, vol. 309, no. 5733, pp. 388-390, 2005. 
[30] T. Mizuno, J. Okumtura, and A. Toriumi, "Experimental study of threshold voltage fluctuation due to statistical variation of channel dopant number in mosfet's," IEEE Trans. Electron Dev., vol. 41, p. 2216, 1994.

[31] Y. Li and C.-H. Hwang, "Discrete-dopant-induced characteristic fluctuations in 16 nm multiplegate silicon-on-insulator devices,” J. Appl. Phys., vol. 102, pp. 084509-6, Oct. 2007.

[32] G. A. Prinz, “Magnetoelectronics,” Science, vol. 282, no. 5394, pp. 1660-1663, 1998.

[33] S. A. Wolf, D. D. Awschalom, R. A. Buhrman, J. M. Daughton, S. von Molnar, M. L. Roukes, A. Y. Chtchelkanova, and D. M. Treger, "Spintronics: A spin-based electronics vision for the future," Science, vol. 294, pp. 1488-1495, Nov. 2001.

[34] S. Datta and B. Das, "Electronic analog of the electro-optic modulator," Appl. Phys. Lett., vol. 56, pp. 665-667, Feb. 1990.

[35] R. Winkler and M. Oestreich, “Spinelektronik,” Physik Journal, vol. 1, p. 39, 2004.

[36] G. Schmidt, D. Ferrand, L. W. Molenkamp, A. T. Filip, and B. J. van Wees, "Fundamental obstacle for electrical spin injection from a ferromagnetic metal into a diffusive semiconductor," Phys. Rev. B, vol. 62, pp. R4790, Aug. 2000.

[37] D. L. Smith and R. N. Silver, "Electrical spin injection into semiconductors," Phys. Rev. B, vol. 64, pp. 045323, July 2001.

[38] A. Fert and H. Jaffrès, "Conditions for efficient spin injection from a ferromagnetic metal into a semiconductor,” Phys. Rev. B, vol. 64, pp. 184420, Oct. 2001.

[39] D. O. Demchenko and A. Y. Liu, "Influence of interface structure on electronic properties and schottky barriers in Fe/GaAs magnetic junctions,” Phys. Rev. B, vol. 73, pp. 115332-9, Mar. 2006.

[40] P. M. Thibado, E. Kneedler, B. T. Jonker, B. R. Bennett, B. V. Shanabrook, and L. J. Whitman, "Nucleation and growth of Fe on GaAs(001)-(2 x 4) studied by scanning tunneling microscopy," Phys. Rev. B, vol. 53, pp. R10481, Apr. 1996.

[41] B. D. Schultz, H. H. Farrell, M. M. R. Evans, K. Ludge, and C. J. Palmstrom, "ErAs interlayers for limiting interfacial reactions in Fe/GaAs(100) heterostructures,” J. Vac. Sci. Technol. B, vol. 20, pp. 1600-1608, July 2002.

[42] H.-P. Schonherr, R. Notzel, W. Ma, and K. H. Ploog, "Evolution of the surface morphology of Fe grown on GaAs (100), (311)a, and (331)a substrates by molecular beam epitaxy,” J. Appl. Phys., vol. 89, pp. 169-173, Jan. 2001. 
[43] G. Wastlbauer and J. A. C. Bland, "Structural and magnetic properties of ultrathin epitaxial Fe films on GaAs(001) and related semiconductor substrates," Advances in Physics, vol. 54, no. 2, pp. 137-219, 2005.

[44] J. W. Freeland, I. Coulthard, W. J. Antel, and A. P. J. Stampfl, "Interface bonding for Fe thin films on GaAs surfaces of differing morphology,” Phys. Rev. B, vol. 63, pp. 193301, Apr. 2001.

[45] A. Filipe, A. Schuhl, and P. Galtier, "Structure and magnetism of the Fe/GaAs interface," Appl. Phys. Lett., vol. 70, pp. 129-131, Jan. 1997.

[46] B. Lepine, S. Ababou, A. Guivarc'h, G. Jezequel, S. Deputier, R. Guerin, A. Filipe, A. Schuhl, F. Abel, C. Cohen, A. Rocher, and J. Crestou, "Solid state interdiffusions in epitaxial Fe/GaAs(001) heterostructures during ultrahigh vacuum annealings up to $450^{\circ} \mathrm{C}$,” J. Appl. Phys., vol. 83, pp. 30773080, Mar. 1998.

[47] J. M. LeBeau, Q. O. Hu, C. J. Palmstrom, and S. Stemmer, "Atomic structure of postgrowth annealed epitaxial Fe/(001)GaAs interfaces,” Appl. Phys. Lett., vol. 93, pp. 121909-3, Sept. 2008.

[48] S. C. Erwin, S.-H. Lee, and M. Scheffler, "First-principles study of nucleation, growth, and interface structure of Fe/GaAs,” Phys. Rev. B, vol. 65, pp. 205422-, May 2002.

[49] S. Mirbt, B. Sanyal, C. Isheden, and B. Johansson, "First-principles calculations of Fe on GaAs(100),” Phys. Rev. B, vol. 67, pp. 155421-, Apr. 2003.

[50] B. Jonker, S. Erwin, A. Petrou, and A. Petukhov, "Electrical spin injection and transport in semiconductor spintronic devices,” MRS Bulletin, vol. 28, p. 740, 2003.

[51] H. J. Zhu, M. Ramsteiner, H. Kostial, M. Wassermeier, H.-P. Schönherr, and K. H. Ploog, “Room-temperature spin injection from Fe into GaAs,” Phys. Rev. Lett., vol. 87, no. 1, p. 016601, 2001.

[52] A. T. Hanbicki, B. T. Jonker, G. Itskos, G. Kioseoglou, and A. Petrou, "Efficient electrical spin injection from a magnetic metal/tunnel barrier contact into a semiconductor,” Appl. Phys. Lett., vol. 80, pp. 1240-1242, Feb. 2002.

[53] A. Hirohata, S. J. Steinmueller, W. S. Cho, Y. B. Xu, C. M. Guertler, G. Wastlbauer, J. A. C. Bland, and S. N. Holmes, "Ballistic spin filtering across ferromagnet/semiconductor interfaces at room temperature,” Phys. Rev. B, vol. 66, pp. 035330, July 2002.

[54] L. Winking, M. Wenderoth, J. Homoth, S. Siewers, and R. G. Ulbrich, "Fe films grown on GaAs(110) in a two-step process: Improved structural and magnetic properties,” Appl. Phys. Lett., vol. 92, pp. 193102-3, May 2008.

[55] W. Moench, Semiconductor Surfaces and Interfaces. Berlin: Springer, 3rd ed., 1995. 
[56] E. H. Rhoderick and R. H. Williams, Metal-Semiconductor Contacts. Oxford: Clarendon, 2nd ed., 1988.

[57] T. J. Zega, A. T. Hanbicki, S. C. Erwin, I. Zutic, G. Kioseoglou, C. H. Li, B. T. Jonker, and R. M. Stroud, "Determination of interface atomic structure and its impact on spin transport using zcontrast microscopy and density-functional theory,” Phys. Rev. Lett., vol. 96, pp. 196101-4, May 2006.

[58] W. E. Meyerhof, "Contact potential difference in silicon crystal rectifiers," Phys. Rev., vol. 71, pp. 727, May 1947.

[59] J. Tersoff, “Calculation of schottky barrier heights from semiconductor band structures," Surface Science, vol. 168, pp. 275-284, Mar. 1986.

[60] J. J. Rehr and W. Kohn, "Impurity states between two bands,” Phys. Rev. B, vol. 9, pp. 1981, Feb. 1974.

[61] W. Moench, "Mechanisms of schottky-barrier formation in metal-semiconductor contacts," $J$. Vac. Sci. Technol. B, vol. 6, pp. 1270-1276, July 1988.

[62] W. E. Spicer, I. Lindau, P. Skeath, and C. Y. Su, “Unified defect model and beyond,” J. Vac. Sci. Technol., vol. 17, pp. 1019-1027, Sept. 1980.

[63] P. Ebert, "Nano-scale properties of defects in compound semiconductor surfaces," Surface Science Reports, vol. 33, no. 4-8, pp. 121-303, 1999.

[64] E. R. Weber, H. Ennen, U. Kaufmann, J. Windscheif, J. Schneider, and T. Wosinski, "Identification of $\mathrm{As}_{\mathrm{Ga}}$ antisites in plastically deformed GaAs,” J. Appl. Phys., vol. 53, pp. 6140-6143, Sept. 1982.

[65] A. Zur, T. C. McGill, and D. L. Smith, "Fermi-level position at a semiconductor-metal interface,” Phys. Rev. B, vol. 28, pp. 2060, Aug. 1983.

[66] W. Moench, "Chemisorption-induced defects at interfaces on compound semiconductors," Surface Science, vol. 132, pp. 92-121, Sept. 1983.

[67] C. J. Chen, Introduction to Scanning Tunneling Microscopy. Oxford: Oxford University Press, 1993.

[68] R. Wiesendanger, Scanning Probe Microcopy and Spectroscopy. Cambridge University Press, 1994.

[69] D. A. Bonnell, Scanning Tunneling Microscopy and Spectroscopy, Theory, Techniques, and Applications. VCH, New York, 1993. 
[70] G. Binnig and H. Rohrer, "Scanning tunneling microscopy - from birth to adolescence," Rev. Mod. Phys., vol. 59, pp. 615, July 1987.

[71] P. Ebert, B. Engels, P. Richard, K. Schroeder, S. Blügel, C. Domke, M. Heinrich, and K. Urban, "Contribution of surface resonances to scanning tunneling microscopy images: (110) surfaces of III-V semiconductors,” Phys. Rev. Lett., vol. 77, no. 14, p. 2997, 1996.

[72] R. M. Feenstra and J. A. Stroscio, “Tunneling spectroscopy of the GaAs(110) surface,” J. Vac. Sci. Technol. B, vol. 5, no. 4, p. 923, 1987.

[73] A. J. Heinrich, Ordering in Ternary Compound Semiconductors on the Atomic Scale. PhD thesis, Universtät Göttingen, Göttingen, 1998.

[74] J. Bardeen, “Tunneling from a many-particle point of view,” Phys. Rev. Lett., vol. 6, p. 57, 1961.

[75] J. Tersoff and D. R. Hamann, “Theory and application for the scanning tunneling microscope,” Phys. Rev. Lett., vol. 50, p. 1998, 1983.

[76] J. Tersoff and D. R. Hamann, "Theory of the scanning tunneling microscope," Phys. Rev. B, vol. 31, p. 805, 1985.

[77] R. J. Hamers and D. F. Padowitz, "Methods of tunneling spectroscopy with the STM," in Scanning Probe Microscopy and Spectroscopy (D. Bonnell, ed.), (New York), Wiley-VCH, 2001.

[78] R. M. Feenstra, J. A. Stroscio, J. Tersoff, and A. P. Fein, "Atom-selective imaging of the GaAs(110) surface,” Phys. Rev. Lett., vol. 58, pp. 1192, Mar. 1987.

[79] H. Schleiermacher, “Charakterisierung von Spitzen für die Rastertunnelmikroskopie,” Master's thesis, Universität Göttingen, 2006.

[80] S. Loth, Atomic scale images of acceptors in III-V semiconductors: band bending, tunneling paths and wave functions. PhD thesis, Universität Göttingen, 2007.

[81] T. C. G. Reusch, M. Wenderoth, A. J. Heinrich, K. J. Engel, N. Quaas, K. Sauthoff, R. G. Ulbrich, E. R. Weber, K. Uchida, and W. Wegscheider, "Influence of short-range ordering on roughness of (AlGa)As interfaces studied with cross-sectional scanning tunneling microscopy,” Appl. Phys. Lett., vol. 76, pp. 3882-3884, June 2000.

[82] C. Ropers, M. Wenderoth, L. Winking, T. C. G. Reusch, M. Erdmann, R. G. Ulbrich, M. Grochol, F. Grosse, R. Zimmermann, S. Malzer, and G. H. Dohler, "Atomic scale structure and optical emission of $\mathrm{Al}_{\mathrm{x}} \mathrm{Ga}_{1}{ }_{-} \mathrm{As} / \mathrm{GaAs}$ quantum wells,” Phys. Rev. B, vol. 75, pp. 115317-7, Mar. 2007. 
[83] L. Winking, M. Wenderoth, T. C. G. Reusch, R. G. Ulbrich, P.-J. Wilbrandt, R. Kirchheim, S. Malzer, and G. Dohler, "Ideal delta doping of carbon in GaAs,” J. Vac. Sci. Technol. B, vol. 23, pp. 267-270, Jan. 2005.

[84] K. Besocke, “An easily operable scanning tunneling microscope,” Surf. Sci., vol. 181, p. 145, 1987.

[85] J. Müller, “Herstellung und Charakterisierung ultradünner Cu-filme auf GaAs(110)-Spaltflächen für Ladungstransportmessungen mit dem Rastertunnelmikroskop,” Master's thesis, Universität Göttingen, Göttingen, 2003.

[86] M. A. Rosentreter, Rastertunnelmikroskopie auf der GaAs(110)-Oberfläche bei Temperaturen von 8 K - 300 K. PhD thesis, Universtät Göttingen, Göttingen, 1997.

[87] K. Sauthoff, “Nichtlineare dynamische Instabilität beim spröden Bruch von GaAs,” Master's thesis, Universität Göttingen, 1998.

[88] D. J. Chadi, “(110) surface atomic structures of covalent and ionic semiconductors,” Phys. Rev. B, vol. 19, pp. 2074-, Feb. 1979.

[89] J. R. Chelikowsky and M. L. Cohen, "Self-consistent pseudopotential calculation for the relaxed (110) surface of GaAs,” Phys. Rev. B, vol. 20, no. 10, p. 4150, 1979.

[90] B. Engels, P. Richard, K. Schroeder, S. Blügel, P. Ebert, and K. Urban, “Comparison between ab initio theory and scanning tunneling microscopy for (110) surfaces of III-V semiconductors,” Phys. Rev. B, vol. 58, pp. 7799, Sept. 1998.

[91] G. J. de Raad, D. M. Bruls, P. M. Koenraad, and J. H. Wolter, "Interplay between tip-induced band bending and voltage-dependent surface corrugation on gaas(110) surfaces,” Phys. Rev. B, vol. 66, pp. 195306, Nov. 2002.

[92] P. M. Koenraad, M. Kemerink, and P. Offermanns, "Calculation of iv-curves measured with stm, copyright p.koenraad ibm 1990.” private communication, 1990.

[93] T. C. G. Reusch, Cross-sectional Scanning Tunneling Microscopy of Au contacts on GaAs(110). PhD thesis, Universität Göttingen, 2003.

[94] K. Besocke and H. Wagner, “Adsorption of W on W(110): Work-function reduction and island formation,” Phys. Rev. B, vol. 8, pp. 4597-, Nov. 1973.

[95] K. Teichmann, “Rastertunnelspektroskopie an Silizium-Donatoren in Galliumarsenid,” Master's thesis, Universität Göttingen, 2007. 
[96] M. C. Payne and J. C. Inkson, "Measurement of workfunctions by tunnelling and the effect of the image potential,” Surface Science, vol. 159, pp. 485-495, Aug. 1985.

[97] R. M. Feenstra, "Electrostatic potential for a hyperbolic probe tip near a semiconductor," J. Vac. Sci. Technol. B, vol. 21, pp. 2080-2088, Sept. 2003.

[98] M. Kemerink, T. C. G. Reusch, D. M. Bruls, P. M. Koenraad, H. W. M. Salemink, and J. H. Wolter, "Quantitative determination of the charge density on surface steps on semiconductors by highresolution local scanning-tunneling spectroscopy,” Physica E, vol. 13, p. 1159, 2002.

[99] T. C. G. Reusch, M. Wenderoth, L. Winking, N. Quaas, and R. G. Ulbrich, "Nanoscale mapping of the space charge layer of Au/GaAs(110) contacts,” Appl. Phys. Lett., vol. 87, pp. 093103-3, Aug. 2005.

[100] K. Sauthoff, Elektronische Eigenschaften von einzelnen Donator und Donatorkomplexen nahe der GaAs(110)-Oberfläche bei 8K. PhD thesis, Universität Göttingen, Göttingen, 2003.

[101] T. C. G. Reusch, M. Wenderoth, L. Winking, N. Quaas, and R. G. Ulbrich, "Origin of schottky barriers in gold contacts on GaAs(110),” Phys. Rev. Lett., vol. 93, pp. 206801, Nov. 2004.

[102] K. B. Kahen, “Theory of schottky-contact formation on GaAs(110),” Phys. Rev. B, vol. 43, pp. 11745, May 1991.

[103] N. Newman, M. van Schilfgaarde, T. Kendelwicz, M. D. Williams, and W. E. Spicer, "Electrical study of schottky barriers on atomically clean GaAs(110) surfaces,” Phys. Rev. B, vol. 33, pp. 1146, Jan. 1986.

[104] G. D. Waddill, I. M. Vitomirov, C. M. Aldao, and J. H. Weaver, "Cluster deposition on GaAs(110): Formation of abrupt, defect-free interfaces,” Phys. Rev. Lett., vol. 62, pp. 1568, Mar. 1989.

[105] J. R. Waldrop and R. W. Grant, "Interface chemistry of metal-GaAs schottky-barrier contacts," Appl. Phys. Lett., vol. 34, pp. 630-632, May 1979.

[106] R. H. Williams, "Surface defect effects on schottky barriers,” J. Vac. Sci. Technol., vol. 18, pp. 929-936, Apr. 1981.

[107] D. M. Hill, F. Xu, Z. Lin, and J. H. Weaver, "Atomic distributions across metal-III-Vcompound-semiconductor interfaces,” Phys. Rev. B, vol. 38, pp. 1893, July 1988.

[108] F. Schäffler, G. Hughes, W. Drube, R. Ludeke, and F. J. Himpsel, “Comparative study of Y and other transition metals on GaAs(110),” Phys. Rev. B, vol. 35, pp. 6328, Apr. 1987.

[109] K. Stiles, A. Kahn, D. G. Kilday, and G. Margaritondo, "Initial stages of schottky barrier formation: Temperature effects,” J. Vac. Sci. Technol. B, vol. 5, pp. 987-991, July 1987. 
[110] G. A. Prinz and J. J. Krebs, "Molecular beam epitaxial growth of single-crystal Fe films on GaAs,” Appl. Phys. Lett., vol. 39, pp. 397-399, Sept. 1981.

[111] A. R. Smith, K.-J. Chao, Q. Niu, and C.-K. Shih, "Formation of atomically flat silver films on GaAs with a "silver mean" quasi periodicity,” Science, vol. 273, pp. 226-228, July 1996.

[112] J. Muller, M. Wenderoth, N. Quaas, T. C. G. Reusch, and R. G. Ulbrich, "Evolution of thin Cu films on GaAs(110) towards atomically flat epitaxial overlayers,” Appl. Phys. Lett., vol. 85, pp. 2220 2222, Sept. 2004.

[113] N. Quaas, Scanning Tunnelling Microscopy of Co-impurified Noble Metal Surfaces: KondoEffect, Electronic Surface States and Diffusional Atom Transport. PhD thesis, Universität Göttingen, 2003. PhD Thesis.

[114] A. Weismann, "Rastertunnelspektroskopie auf magnetisch verunreinigten $\mathrm{Cu}$ - und AgEinkristalloberflächen,” Master's thesis, Universität Göttingen, Göttingen, 2003.

[115] G. A. Prinz, G. T. Rado, and J. J. Krebs, "Magnetic properties of single-crystal 110 iron films grown on GaAs by molecular beam epitaxy (invited),” J. Appl. Phys., vol. 53, pp. 2087-2091, Mar. 1982.

[116] M. W. Ruckman, J. J. Joyce, and J. H. Weaver, "Interdiffusion and reaction at the Fe/GaAs(110) interface,” Phys. Rev. B, vol. 33, pp. 7029, May 1986.

[117] P. N. First, J. A. Stroscio, R. A. Dragoset, D. T. Pierce, and R. J. Celotta, "Metallicity and gap states in tunneling to fe clusters in GaAs(110),” Phys. Rev. Lett., vol. 63, pp. 1416, Sept. 1989.

[118] R. Hollinger, M. Zolfl, R. Moosbuhler, and G. Bayreuther, "In-plane spin reorientation transitions in epitaxial Fe(110)/GaAs(110) films,” J. Appl. Phys., vol. 89, pp. 7136-7138, June 2001.

[119] J. J. Krebs, B. T. Jonker, and G. A. Prinz, "Properties of Fe single-crystal films grown on (100)GaAs by molecular-beam epitaxy,” J. Appl. Phys., vol. 61, pp. 2596-2599, Apr. 1987.

[120] F. A. Volkening, B. T. Jonker, G. A. Prinz, and N. C. Koon, "Moessbauer study of ${ }^{57}$ Fe singlecrystal films grown on (110) GaAs by MBE,” J. Appl. Phys., vol. 67, pp. 5646-5648, May 1990.

[121] Y. B. Xu, E. T. M. Kernohan, D. J. Freeland, A. Ercole, M. Tselepi, and J. A. C. Bland, "Evolution of the ferromagnetic phase of ultrathin Fe films grown on GaAs(100)-4 x 6," Phys. Rev. B, vol. 58, pp. 890, July 1998.

[122] J. Bland, A. Hirohata, Y.-B. Xu, C. Guertler, and S. Holmes, "Spin-polarized electron transport processes at the ferromagnet/semiconductor interface,” IEEE Trans. Magn., vol. 36, p. 2827, 2000. 
[123] D. A. Hite, S. E. Russek, and D. P. Pappas, "In situ conductance characterization of Fe/Ag multilayer contacts on GaAs,” J. Appl. Phys., vol. 94, pp. 621-625, July 2003.

[124] C. H. Li, G. Kioseoglou, O. M. J. van 't Erve, A. T. Hanbicki, B. T. Jonker, R. Mallory, M. Yasar, and A. Petrou, "Spin injection across (110) interfaces: Fe/GaAs(110) spin-light-emitting diodes," Appl. Phys. Lett., vol. 85, pp. 1544-1546, Aug. 2004.

[125] D. Lyzwa, “Construction d'une chambre a vide pour mesurer l'effet kerr magneto-optique,” Master's thesis, Universite Pierre et Marie Curie, Paris 6, 2006.

[126] M. Gester, C. Daboo, R. J. Hicken, S. J. Gray, A. Ercole, and J. A. C. Bland, “Continuous evolution of the in-plane magnetic anisotropies with thickness in epitaxial Fe films,” J. Appl. Phys., vol. 80, pp. 347-355, July 1996.

[127] E. C. Stoner and E. P. Wohlfarth, “A mechanism of magnetic hystersis in heterogeneous alloys,” Phil. Trans. R. Soc., vol. 240, p. 599, 1948.

[128] Y. Chye, V. Huard, M. E. White, and P. M. Petroff, "Properties of a Fe/GaAs(001) hybrid structure grown by molecular-beam epitaxy,” Appl. Phys. Lett., vol. 80, pp. 449-451, Jan. 2002.

[129] C. Domke, P. Ebert, M. Heinrich, and K. Urban, "Microscopic identification of the compensation mechanisms in Si-doped GaAs,” Phys. Rev. B, vol. 54, pp. 10288, Oct. 1996.

[130] L. Winking, "Raster-Tunnel-Mikroskopie von Kohlenstoff-Akzeptoren in deltadotiertem Gallium-Arsenid,” Master's thesis, Universität Göttingen, 2003.

[131] R. W. Jansen and O. F. Sankey, “Theory of relative native- and impurity-defect abundances in compound semiconductors and the factors that influence them,” Phys. Rev. B, vol. 39, pp. 3192, Feb. 1989.

[132] M. C. M. M. van der Wielen, A. J. A. van Roij, and H. van Kempen, "Direct observation of friedel oscillations around incorporated $\mathrm{Si}_{\mathrm{Ga}}$ dopants in $\mathrm{GaAs}$ by low-temperature scanning tunneling microscopy,” Phys. Rev. Lett., vol. 76, pp. 1075, Feb. 1996.

[133] J. E. Northrup and S. B. Zhang, "Dopant and defect energetics: Si in GaAs," Phys. Rev. B, vol. 47, pp. 6791, Mar. 1993.

[134] G. Cox, K. Graf, D. Szynka, U. Poppe, and K. Urban, "Observation of point defects and microfaceting on GaAs(110) surfaces by scanning tunneling microscopy,” Vacuum, vol.41, no.1-3, pp. 591-595, 1990.

[135] S. M. Sze, Physics of Semiconductor Devices. New York: Wiley-Interscience, 1981. 
[136] H.-J. Im, Y. Ding, J. P. Pelz, and W. J. Choyke, “Nanometer-scale test of the tung model of schottky-barrier height inhomogeneity,” Phys. Rev. B, vol. 64, pp. 075310, July 2001.

[137] K. Teichmann, M. Wenderoth, S. Loth, R. G. Ulbrich, J. K. Garleff, A. P. Wijnheijmer, and P. M. Koenraad, "Controlled charge switching on a single donor with a scanning tunneling microscope," Phys. Rev. Lett., vol. 101, pp. 076103-4, Aug. 2008.

[138] A. B. McLean, R. H. Williams, and J. F. McGilp, "Metal d-level induced mid-gap fermi level pinning on GaAs(110),” J. Vac. Sci. Technol. B, vol. 6, pp. 1252-1256, July 1988.

[139] A. B. McLean and R. H. Williams, "Schottky contacts to cleaved GaAs(110) surfaces. i. electrical properties and microscopic theories,” Journal of Physics C: Solid State Physics, vol. 21, no. 4, pp. 783-806, 1988.

[140] W. Moench, "Chemical trends in schottky barriers: Charge transfer into adsorbate-induced gap states and defects,” Phys. Rev. B, vol. 37, pp. 7129, Apr. 1988.

[141] W. Moench, "On the physics of metal-semiconductor interfaces," Reports on Progress in Physics, vol. 53, no. 3, pp. 221-278, 1990.

[142] W. Moench, "Metal-semiconductor contacts: electronic properties,” Surface Science, vol. 299300, pp. 928-944, Jan. 1994.

[143] R. L. Anderson, "Experiments on Ge-GaAS heterojunctions,” Solid-State Electronics, vol. 5, no. 5, pp. 341-344, 1962.

[144] F. Bensch, G. Garreau, R. Moosbuhler, G. Bayreuther, and E. Beaurepaire, "Onset of ferromagnetism in Fe epitaxially grown on $\operatorname{GaAs}(001)(4 \times 2)$ and $(2 \times 6)$,” J. Appl. Phys., vol. 89, pp. 7133-7135, June 2001.

[145] G. Binnig, N. Garcia, H. Rohrer, J. M. Soler, and F. Flores, "Electron-metal-surface interaction potential with vacuum tunneling: Observation of the image force,” Phys. Rev. B, vol. 30, pp. 4816, Oct. 1984. 


\section{Publikationen}

1. L. Winking, M. Wenderoth, J. Homoth, S. Siewers R.G. Ulbrich, Fe films grown on GaAs(110) in a two-step process: Improved structural and magnetic properties, Appl. Phys. Lett. 92193102 (2008)

2. C. Ropers, M. Wenderoth, L. Winking, T.C.G. Reusch, M. Erdmann, R.G. Ulbrich, M. Grochol, F. Grosse, R. Zimmerman, S. Malzer, G.H. Döhler, Atomic scale structure and optical emission of $A l_{x} G a_{1-x} A s / G a A s$ quantum wells, Phys. Rev. B 75, p.115317 (2007)

3. S. Loth, M. Wenderoth, L. Winking, R. G. Ulbrich, S. Malzer, G.H. Döhler, Probing semiconductor gap states with resonant tunnelling, Phys. Rev. Lett. 96, p.066403 (2006)

4. S. Loth, M. Wenderoth, L. Winking, R. G. Ulbrich, S. Malzer, G.H. Döhler, Depth Resolved Scanning Tunneling Spectroscopy of shallow acceptors in Gallium Arsenide, Jap. Journ. Appl. Phys. 45, p.2193-2196 (2006)

5. L. Winking, M. Wenderoth, T.C.G. Reusch, R.G. Ulbrich, P.-J. Wilbrandt, R. Kirchheim, S. Malzer and G. Döhler, Structural properties and electronic states of carbon delta-layers in GaAs, $27^{\text {th }}$ International Conference on the Physics of Semiconductors, edited by J. Menendez and C. van de Walle, AIP Conference Proceedings, Melville, New York, p.151 (2005)

6. L. Winking, M. Wenderoth, T.C.G. Reusch, R.G. Ulbrich, P.-J. Wilbrandt, R. Kirchheim, S. Malzer and G. Döhler, Ideal Delta doping of carbon in GaAs, J. Vac. Sci. Technol. B 23, p.267 (2005)

7. T.C.G. Reusch, M. Wenderoth, L. Winking, N. Quaas, and R.G. Ulbrich, Nano-scale mapping of the space charge layer of Au/GaAs(110) contacts, Appl. Phys. Lett. 87, p.093103 (2005)

8. T.C.G. Reusch, M. Wenderoth, L. Winking, N. Quaas, and R.G. Ulbrich, Origin of Schottky Barriers in Gold Contacts on GaAs(110), Phys. Rev. Lett. 93, p.206801 (2004)

\section{Präsentationen}

1. Lars Winking, Martin Wenderoth, Jan Homoth, Swante Sievers, and Rainer G. Ulbrich, Real space imaging of the Fe/GaAs(110) interface with Cross-Sectional Scanning Tunneling Microscopy, Frühjahrstagung der DPG in Berlin (2008)

2. Lars Winking, Martin Wenderoth, Swante Sievers, Jan Homoth, and Rainer G. Ulbrich, Magnetic properties of thin Fe films grown on GaAs(110) in a two-step process, Frühjahrstagung der DPG in Berlin (2008)

3. Lars Winking, Martin Wenderoth, Jan Homoth, Rainer G. Ulbrich, Epitaxial growth of thin and atomically flat Fe-films on GaAs(110) at $L N_{2}$ temperature, Frühjahrstagung der DPG in Dresden (2006) 
4. Lars Winking, Martin Wenderoth, Jan Homoth, Rainer G. Ulbrich, Thin epitaxial and atomically flat Fe-films grown on GaAs(110) in a two-step process, $24^{\text {th }}$ European Conference on Surface Science (ECOSS) in Paris (2006)

5. Lars Winking, Martin Wenderoth, Jan Homoth, and Rainer G. Ulbrich, Two-step process for epitaxial growth of thin and atomically flat Fe-films on GaAs(110), $28^{\text {th }}$ International Conference on the Physics of Semiconductors (ICPS) in Wien (2006)

6. Lars Winking, Martin Wenderoth, Jan Müller, Rainer G. Ulbrich, Growth of thin and atomically flat Fe-films on GaAs(110), Frühjahrstagung der DPG in Berlin (2005)

7. L. Winking, M. Wenderoth, T.C.G. Reusch, R.G. Ulbrich, P.-J. Wilbrandt, R. Kirchheim, S. Malzer and G. Döhler, Structural Properties And Electronic States Of Carbon Delta-Layers In GaAs, $27^{\text {th }}$ International Conference on the Physics of Semiconductors (ICPS) in Flagstaff (2004)

8. L. Winking, M. Wenderoth, T.C.G. Reusch, R.G. Ulbrich, P.-J. Wilbrandt, R. Kirchheim, S. Malzer and G. Döhler, Distribution of C-acceptors in delta-doped GaAs studied with Cross-sectional Scanning Tunneling Microscopy, Frühjahrstagung der DPG in Regensburg (2004)

9. L. Winking, M. Wenderoth, T.C.G. Reusch, R.G. Ulbrich, P.-J. Wilbrandt, R. Kirchheim, S. Malzer and G. Döhler, Ideal delta-doping of carbon in GaAs, $8^{\text {th }}$ International Conference on NanometerScale Science and Technology (Nano8) in Venedig (2004)

10. T.C.G. Reusch, M. Wenderoth, L. Winking, R.G. Ulbrich, G. Döhler, S. Malzer, Cross-sectional scanning tunnelling microscopy of C-acceptors in GaAs and their properties in delta-doped layers, $3^{\text {rd }}$ International Conference of Scanning Tunnelling Microscopy (STM) in Eindhoven (2003)

11. T.C.G. Reusch, M. Wenderoth, L. Winking, R.G. Ulbrich, S. Malzer und G. Döhler, Eigenschaften von C-Delta-Dotierschichten in GaAs auf der atomaren Skala, Frühjahrstagung der DPG in Dresden (2003) 


\section{Danksagung}

All denjenigen, die auf ihre Art persönlich und auch fachlich zum Gelingen dieser Arbeit beigetragen haben, sei an dieser Stelle herzlich gedankt. Darüber hinaus möchte ich die Gelegenheit nutzen, um einige besonders hervorzuheben.

An erster Stelle möchte ich Prof. Dr. R. G. Ulbrich nennen, der mein Studium seit dem ersten Tag in Göttingen begleitet hat - zunächst in Form der Anfängervorlesung, später in verschiedenen Seminaren bis hin zur aktiven Mitarbeit in seiner Arbeitsgruppe. Seine Diskussionsbereitschaft und seine Denkanstöße haben wesentlich zum erfolgreichen Abschluss dieser Arbeit beigetragen.

Prof. Dr. R. Kirchheim danke ich für die Übernahme des Korreferats. Für ihre Mitarbeit in der Prüfungskommission danke ich Prof. Dr. Ch. Jooß, Prof. Dr. H. Hofsäss, Prof. Dr. F. Kneer und Prof. Dr. W. Glatzel.

Besonderer Dank gilt Dr. M. Wenderoth, der in vielen fruchtbaren Diskussionen entscheidend an der Entstehung dieser Arbeit mitgewirkt hat. Seine offene und herzliche Art hat wesentlich dazu beigetragen, eine ungezwungene, freundschaftliche und zugleich motivierende Atmosphäre in der Arbeitsgruppe zu erzeugen, die das Arbeiten sehr angenehm gestaltet hat.

Dem Rest der Tunnelgruppe - Thomas Druga, Tim Iffländer, Jens Garleff, Jan Homoth, Philipp Kloth, Peter Löptien, Karolin Löser, Sebastian Loth, Henning Prüser, Thilo Reusch, Swante Siewers, Thomas Späth, Karen Teichmann, Alexander Weismann - möchte ich für ihren täglichen Beistand in Labor und Büro danken.

Von den Mitgliedern der Arbeitsgruppe möchte ich zum einen Thilo Reusch hervorheben, der mir während meiner Diplomarbeit kompetent und versiert mit Rat und Tat zur Seite stand und mich für die XSTM Untersuchung von Metall-Halbleiter Kontakten begeistert hat.

Zum anderen aber auch ganz besonders Jan Homoth, mit dem ich nicht nur die Zeit vom Studium bis zur Promotion mit seinen Höhen und Tiefen zusammen bestritten habe, sondern auch außerhalb der Uni während der vergangenen Jahre viele unvergessene Momente erlebt habe.

Ein großes Lob geht an Bernhard Spicher für seinen unermüdlichen Einsatz im täglichen Kampf mit den UHV Anlagen. Der Zentralwerkstatt sowie den Teams der Feinmechanik- und Elektronikwerkstatt des IV. Physikalischen Instituts möchte ich für die gute und unkpomlizierte Unterstützung bei allen technischen Problemen des Laboralltags danken.

Vor allem bin ich meiner Familie zutiefst dankbar. Es ist ein gutes Gefühl zu wissen, dass ich mich in allen Lebenslagen auf ihre Unterstützung und unseren engen Zusammenhalt verlassen kann. 


\title{
Lebenslauf
}

\author{
Lars-Helge Winking \\ Diplom-Physiker \\ geboren am 10.01.1978 in Lübeck \\ Staatsangehörigkeit: Deutschland
}

$01 / 2009$

Promotion zur Erlangung des Doktorgrades der Naturwissenschaften (Institut für Halbleiterphysik, Georg-August-Universität Göttingen), Thema: "Untersuchung der lokalen strukturellen und elektronischen Eigenschaften von Fe-GaAs Schottky-Kontakten mit atomar aufgelöster Raster-TunnelMikroskopie in Querschnittsgeometrie“

$05 / 2003$

Diplom im Fach Physik (Georg-August-Universität Göttingen), Schwerpunkt Festkörperphysik

\section{Abschlussnote: sehr gut}

06/2002 - 04/2003 Diplomarbeit (Institut für Halbleiterphysik, Georg-August-Universität Göttingen), Thema: „Raster-Tunnel-Mikroskopie von Kohlenstoff-Akzeptoren in deltadotiertem Gallium-Arsenid“

\section{Abschlussnote: 1,0}

10/1998 - 03/2003 Diplomstudiengang Physik (Georg-August-Universität Göttingen), 9 Fachsemester

08/1997 - 09/1998 Ersatzdienst im „Centre Mediterranéen du Cap d’Ail“, Frankreich, getragen durch Officium Bonum e.V. / Deutsch-Französisches Jugendwerk

05/1997

\section{Allgemeine Hochschulreife}

\section{Abschlussnote: 1,5}

09/1988 - 06/1997 Gymnasium „Johanneum zu Lübeck“ (Lübeck)

09/1984 - 07/1988 Grundschule „Waldschule“ (Groß Grönau bei Lübeck) 